


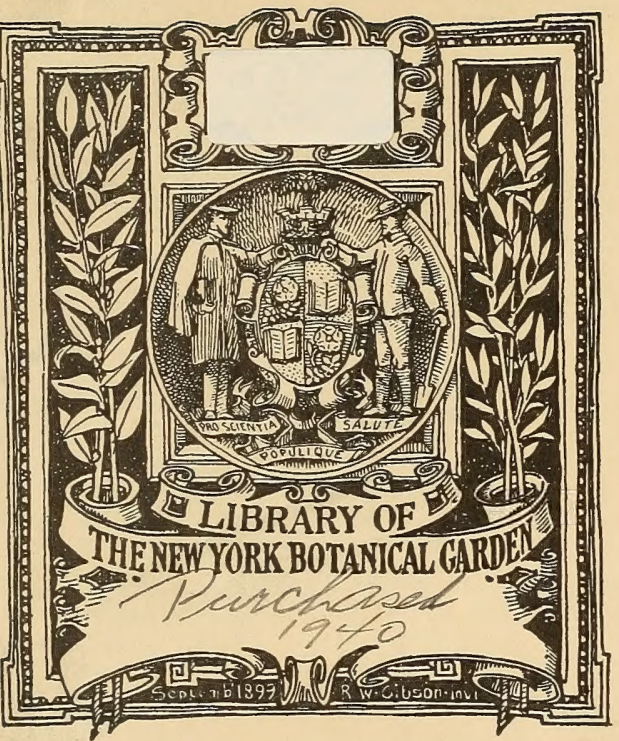





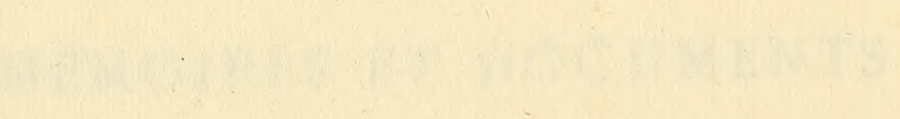




\section{COL LECTION \\ DE \\ MÉMOIRES ET DOCUMENTS \\ PUBLIÉS}

PAR ORDRE DE S. A. S. LE PRINCE ALBERT I ${ }^{\text {er }}$

PRINCE SOUVERAIN DE MONACO 


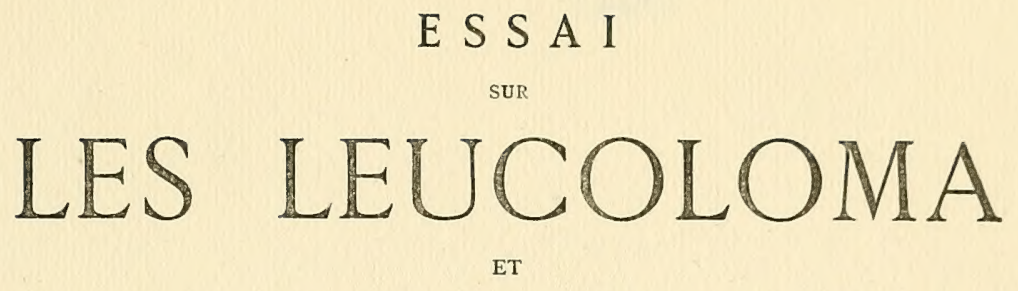

SUPPLÉMENT AU PRODROME

DE LA

\section{FLORE BRYOLOGIQUE \\ DE MADAGASCAR \\ DES M A SCAREIGNES \\ ET DES COMORES}

PUBLIÉS PAR ORDRE DE S. A.S. LE PRINCE ALBERT I ${ }^{\text {Ir }}$

PAR

Ferdinand RENAULD

OUVRAGE COURONNÉ PAR L'INSTITUT DE FRANCE

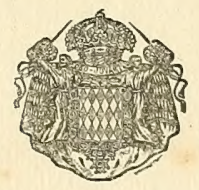

LIBRARY

NEW YORK

BOTANICAL

GARDEQ

IMPRIMERIE DE MONACO 
QK546

. M3

R42

Suppl. 
E S S A I

SUR

\section{LES LEUCOLOMA}



Cette étude est moins une monographie que le travail préparatoire à toute monographie. On ne peut arriver aubut final de la connaissance et de la délimitation des espèces qu'à l'aide de nombreux et riches matériaux d'herbier et d'observations faites sur le vif sur le polymorphisme des espèces et l'étendue de leurs variations. Mais, avant que ces conditions aient pu être réunies, il y a lieu tout d'abord, dans les genres à nombreuses espèces, de rechercher les meilleurs caractères à employer pour les différenciations; de grouper les espèces qui possèdent les mêmes caractères principaux de façon à distinguer des sous-genres ou des sections, ce qui permet de rendre les descriptions spécifiques plus claires et plus précises en les allégeant des caractères de groupe; enfin, dans le cas (qui est celui des Leucoloma) d'espèces souvent síériles, d'étudier les caractères histologiques des organes de végétation, leur accord ou leur divergence avec les caractères morphologiques et le rôle qu'ils doivent remplir dans la classification. 
vIII

Tel est l'objet de ce travail qui nous a permis, par l'examen d'environ 200 Leucoloma, c'est-à-dire de presque toutes les espèces connues, d'aboutir aux résultats suivants:

$\mathrm{I}^{\circ}$ Distinction de 3 genres dans l'ancien genre Leucoloma.

$2^{\circ}$ Division des vrais Leucoloma en 3 sous-genres et en sections.

$3^{\circ}$ Utilisation de l'histologie de la nervure des Dicranolóma pour les différenciations spécifiques et de groupe.

$4^{\circ}$ Accord des données histologiques fournies par le limbe de la feuille et les caractères carpologiques dans les 3 genres; dans les Dicranoloma, indépendance des variations de la structure anatomique de la nervure relativement aux caractères carpologiques.

Je me fais un devoir de payer un tribut de reconnaissance à la mémoire de mon savant et regretté maître et ami M. Bescherelle qui m'a fourni de nombreux et précieux types et de remercier mes amis MM. J. Cardot et V. F. Brotherus qui ont généreusement partagé avec moi leurs collections. Je dois aussi à l'obligeance de M. le $\mathrm{D}^{\mathrm{r}}$ Engler, directeur du Museum de Berlin, communication de plusieurs types provenant des collections de feu le $D^{r}$ Karl Müller; mais déjà je tenais de ce célèbre bryologue quelques espèces africaines et malgaches.

J'ai des obligations particulières à mon ami M. N. Orzeszko, bien connu par ses travaux sur l'histotaxie des Festuca, qui a bien voulu me prêter le secours de son talent de photomicrographe. Grâce à lui j'ai pu obtenir la reproduction très amplifiée de nombreuses coupes, termes précieux 
de comparaison, tandis que les images virtuelles perçues par l'œil dans le microscope s'effacent rapidement de la mémoire. Par le même procédé, j'ai pu fixer par le dessin, avec la plus grande somme possible d'exactitude, des détails anatomiques qu'il ne serait pas aussi facile ni aussi sûr de reproduire à l'aide de la chambre claire.

Je ne terminerai pas sans offrir l'expression respectueuse de ma gratitude à S. A.S. le Prince Albert de Monaco qui a bien voulu admettre ce Mémoire dans la collection des Documents publiés par Son ordre et dont le bienveillant intérêt et le généreux appui s'étendent, au-delà même de Ses admirables travaux océanographiques, à toutes les branches de la Science.

Nice, Mars 1905. 



\section{E S S A I}

SUR

\section{LES LEUCOLOMA}

\section{H ISTORIQUE}

Les bryologues ayant adopté le genre Leucoloma de Bridel (Bryologia universalis, II, p. 2 I $8-1825)$, la citation des synonymes anciens n'offre qu'un intérêt secondaire; aussi je ne reproduis que pour mémoire les noms de: Sclerodontium Schwægr. Suppt II, p. I, p. I 24 (I 823), Macrodon W.A. in Mem. Hist. nat. de Paris, II, p. 299 (1 825), Walkeria Hornsch. in Flora, 1825.

Dans son Synopsis Muscorum frondosorum, I, p. 352 (1849), le $D^{r}$ Karl Müller, qui avait du genre une conception très large,a fait du genre Leucoloma de Bridel une simple section du genre Dicranum et y énumère 7 espèces, les seules connues alors.

Dans ses Musci austro-americani, p. $9^{2}$ (1869), W. Mitten scinde en 2 genres le genre Leucoloma de Bridel. Il ne conserve dans ce dernier qu'une seule espèce : L. mollissinum (Mitt.) et établit le genre Pocilophyllum Mitt., dans lequel il comprend 9 espèces. Les diagnoses de ces 2 genres dans l'ouvrage précité sont si écourtées et si peu significatives qu'il n'est pas facile de s'en former une idée bien nette. On pourrait soupçonner toutefois que, pour Mitten, le genre Leucoloma (sensu stricto) correspondrait au sous-genre Taniodictyon Ren. et le genre 
Pocilophyllum au sous-genre Syncratodictyon Ren. L'examen des espèces énumérées par Mitten prouve en effet que le $L$. mollissimum (Mitt.) est bien du subg. Treniodictyon Ren. et que plusieurs espèces de Pœcilophyllum sont du subg. Syncratodictyon Ren.; mais parmi ces dernières, les $P$. triforme Mitt. et $P$. serrulatum (Brid.) sont du subg. Tæniodictyon Ren.

Si nous sortons du domaine austro-américain, nous trouvons quelques espèces classées par Mitten dans son genre Pœcilophyllum et qui n'appartiennent pas au subg. Syncratodictyon Ren., par exemple $P$. acutum Mitt. (nomen nudum) de Madagascar qui est un Sphenodictyon Ren. et, d'autre part, les $P$. lucinerve Mitt. et $P$. nitens Mitt. de Ceylan qui se réfèrent au subg. Toniodictyon Ren. On peut conclure de ces faits contradictoires que la concordance entre le subg. Syncratodictyon Ren. et le genre Pocilophyllum Mitt. reste douteuse; mais, existerait-elle que ce dernier ne pourrait pas être conservé comme genre. Ni le $\mathrm{D}^{\mathrm{r}}$ Karl Müller, ni Bescherelle, ni M. Brotherus ne l'ont admis, et, de fait, les 'deux sous-genres Toniodictyon Ren. et Syncratodictyon Ren. sont reliés entre eux par les sections Subvittata Ren. et Semivittata Broth.

Plus tard, le $\mathrm{D}^{\mathrm{r}}$ Karl Müller et Bescherelle classèrent dans le genre Leucoloma plusieurs anciens Dicranum à feuilles pourvues d'un margo blanchâtre analogue à celui qui caractérise les Leucoloma. C'est le cas notamment des L. platyloma Besch., L. formosum Besch. de la Nouvelle Calédonie, L. austro scoparium C. M., L. serratum Broth. de l'Australie, L. sumatranum Broth. de Sumatra.

Dans le Prodrome de la Flore bryologique de Madagascar (1897), j'ai distingué 3 sous-genres dans le genre Leucoloma de Bridel:

$I^{\circ}$ Dicranoloma comprenant les anciens Dicranum déclassés ;

$2^{\circ}$ Euleucoloma pour les vrais Leucoloma dans le sens de Bridel;

$3^{\circ}$ Dicnemoloma pour quelques espèces australiennes en partie comprises autrefois dans les Leucoloma.

Le $\mathrm{D}^{\mathrm{r}}$ Karl Müller, dans son Genera muscorum frondosorum(19or), subordonne le genre Leucoloma de Bridel comme sous-genre au genre Dicranum (sensu lato) et il distingue en outre, au même titré, les sousgenres Oncophoroloma C. Müll. et Dicnemonella (Hpe).

Le genre Leucoloma figure dans les premières livraisons du très important ouvrage de M. V. F. Brotherus, en cours de publication : Pflantzen Familien (1901). L'auteur adopte et étend à l'ensemble des espèces de Leucoloma la classification que j’ai publiée pour les espèces 
des îles austro-africaines dans le Prodrome de la Flore bryologique de Madagascar. Il se borne à ajouter une section et découvre que la majeure partie des Dicranum de l'hémisphère austral appartiennent en réalité au sous-genre Dicranoloma.

Enfin, dans la Revue bryologique de M. Husnot (Igor, $n^{\text {os }} 4$ et 5), j'ai conclu à la distinction, comme genres, des 3 sous-genres Dicranoloma, Leucoloma et Dicnemoloma du Prodrome de Madagascar. 


\section{CHAPITRE I}

ANATOMIE

T IGE

Dans les Dicranoloma la tige est pourvue d'un faisceau axile plus ou moins développé, mais toujours visible. Examinée sur une coupe transversale, elle est composée d'un réseau cellulaire à grandes mailles dont les plus dilatées se trouvent autour du faisceau axile. Du centre de la tige où leurs parois sont peu épaisses, en allant vers la périphérie, l'ampleur des cellules diminue progressivement, leurs parois s'épaississent beaucoup et les rangées extérieures forment un anneau scléreux de couleur plus foncée. La rangée épidermique n'est généralement pas différenciée ou l'est très peu.

Dans les vrais Leucoloma et les Dicnemoloma, la tige est dépourvue de faisceau axile, ce qui établit une différence essentielle relativement au genre précédent. Les cellules les plus amples sont au centre, moins dilatées cependant et à parois plus épaisses que dans les Dicranoloma; elles passent insensiblement aux cellules plus petites et plus sclérifiées de la périphérie qui forment un anneau plus large, mais pas mieux délimité, tandis que la rangée épidermique est généralement peu différenciée.

Dans les 3 genres, la tige émet des rhizoïdes plus ou moins abondantes et, comme d'ailleurs dans tous les genres de mousses, sa structure se retrouve identique dans le pédicelle qui est muni ou dépourvu d'un faisceau axile selon que la tige en possède ou n'en a pas.

\section{FEUILLE}

Limbe de la feuille.

Chez les Dicranoloma, le tissu du limbe est uniforme transversalement et ne diffère de celuides Dicranum que par la présence d'un margo hyalin parfois large, d'autres fois étroit ou même peu visible, constitué 
par des rangées de cellules subitement très allongées, à parois épaisses, blanchâtres et à lumen linéaire, à peine apparent.

Dans le plus grand nombre des especes, par ex. D. commutatum (Hpe Broth. du Cap, D. dichotomum (Brid.) Ren. des Mascareignes, D. Billiardieri (Schwgr.) Broth. de l'Australie, D. Blumei (Nees.) Broth. et D.assimile.(Hpe) Broth. de Java, D. formosum (Besch.) Ren. de la Nouvelle Calédonie, etc., le tissu est analogue à celui du Dicranum scoparium L. et composé de cellules allongées à parois légèrement sinueuses et plus ou moins poreuses, dépourvues de papilles et formant une masse souvent un peu scarieuse.

Chez quelques autres espèces, par ex. D. Menziezii (Tayl.) Broth. de la Tasmanie, D. suberectum (Hpe) Broth. et D. serratum Broth. du Queensland, la moitié ou les 2/3 supérieurs de la feuille sont constitués par des cellules courtes, chlorophylleuses, carrées ou brièvement rectangulaires, plus ou moins papilleuses sur la face dorsale; mais que ce soit l'un ou l'autre de ces 2 types de tissu que l'on considère, il y a toujours homogénéité entre les cellules juxtacostales et les cellules juxtamarginales prises au mème niveau.

Il sera parlé, dans le chapitre relatif à la classification, du tissu foliaire du $D$. dicarpum (Brid.) d'Australie qui constitue une exception unique, intermédiaire entre les Dicranoloma et les Leucoloma, et qui correspond peut-être à un type ancien, isolé, en voie de disparition.

Dans les Dicranoloma, les cellules alaires grandes, carrées, rectangulaires ou subhexagones, presque toujours colorées en roux brun ou pourpré, avec vestiges généralement apparents des débris de l'utricule primordial, forment des oreillettes excavées plus ou moins développées. Mais il est inutile d'insister sur ces faits qui reproduisent les mêmes traits que ceux des Dicranum.

Chez les Leucoloma, le tissu foliaire n'est plus uniforme transversalement, mais différencié. $1^{\circ}$ On trouve d'abord, de chaque côté de la nervure, une bande de cellules chlorophylleuses, le plus souvent papilleuses, petites, carrées ou subhexagones, arrondies ou brièvement oblongues, à parois épaisses, formant une lame plus ou moins obscure. Je les appellerai: cellules internes. Elles occupent généralement le tiers ou presque toute la moitié supérieure du limbe de la feuille où elles se trouvent en contact avec le margo hyalin. Tantôt elles forment des bandes juxtacostales régulières, nettement séparées du tissu membraneux contigu et se terminant en pointe ou en coin à une distance plus ou moins grande au-dessus de la base (subgenus Sphenodictyon), ou bien elles descendent 
sans se rétrécir jusqu'à la base ou à peu près (subgenus Taniodictyon) - tantôt sur les côtés, ainsi qu'à une certaine distance au-dessus de la base, elles passent par des transitions insensibles au tissu membraneux qui occupe alors toute la base (subgenus Syncratodictyon).

$2^{\circ}$ Entre les cellules internes juxtacostales et les marges de la feuille se trouve un tissu pâle et serré, sans chlorophylle ni papilles, développé surtout dans la moitié inférieure de la feuille, et qui se compose de cellules linéaires allongées et étroites à parois plus ou moins épaisses. Je les appellerai cellules intermédiaires ou membraneuses parce que, par suite de l'épaisseur de leurs parois, elles semblent souvent confluentes en une masse scarieuse. C'est naturellement dans les sous-genres Sphenodictyon et Syncratodictyon, où les cellules internes chlorophylleuses cessent à une distance notable de la base,que le tissu membraneux a son plus grand développement et occupe entièrement celle-ci.

$3^{\circ}$ Vers les marges de la feuille, les cellules membraneuses, plus ou moins subitement, s'allongent encore, leurs parois s'épaississent à ce point que le lumen est réduit à une ligne à peine perceptible et la feuille est alors bordée par un margo blanchâtre plus ou moins large selon les espèces et qui est la note caractéristique de nos 3 genres relativement aux Dicranum.

Lorsque le passage entre le tissu membraneux et le margo est brusque, celui-ci apparaît très net et même parfois se sépare de lui-même du tissu voisin dans les anciennes feuilles; ailleurs la transition s'opère peu à peu et la largeur du rebord hyalin varie dans une même feuille selon le point où on l'examine; parfois même le margo n'est pas apparent et il faut signaler son absence dans les descriptions; mais, en réalité, il n'est jamais complètement nul et se réduit toujours. au moins à une seule série de cellules plus allongées qui forment le bord de la feuille.

Vues sur une coupe transversale, ces cellules marginales ne diffèrent des cellules membraneuses que par le lumen plus étroit, et encore, le plus souvent, la distinction en est plus difficile à faire sur la coupe que sur la feuille examinée à plat sous le microscope. En revanche, la différence entre les cellules marginales et membraneuses d'une part, et de l'autre les cellules internes ressort très bien sur une coupe transversale. Le lumen des premières est arrondi ou oblong, à grand axe parallèle au plan de la feuille, tandis que la section des cellules internes est plus grande (surtout en se rapprochant de la nervure), le lumen subrectangulaire ou oblong, à grand axe perpendiculaire au plan de la feuille. Il 
en résulte une plus grande épaisseur de la lame chlorophylleuse interne, épaisseur encore augmentée par la protubérance des papilles, et, lorsque la feuille est sèche, il se forme un pli tout lelong de la ligne de contact de la lame interne et de la lame membraneuse. De là le nom de $L$. biplicatum appliqué par Hampe à une espèce du Brésil où cette particularité n'est d'ailleurs pas plus marquée que chez les autres espèces du sous-genre Triniodictron.

Les oreillettes des Leucoloma sont tantôt excavées et semblables à celles des Dicranoloma (subgenus Syncratodictyon), tantôt planes et alors formées de cellules carrées ou rectangulaires articulées bout à bout et d'aspect scalariforme (subg. Treniodictyon et Sphenodictyon).

Chez les Dicnemoloma, comme chez les Leucoloma, le tissu du limbe est différencié, mais la disposition des éléments anatomiques est très différente. Dans les premiers, les cellules internes qui occupent presque tout le limbe descendent le long des marges jusqu'aux oreillettes, tandis qu'un espace juxtacostal restreint est constitué par du tissu membraneux. Cette différence essentielle dans la position respective des deux tissus sépare complètement les 2 genres.

Nervure.

La nervure dans nos 3 genres atteint ou dépasse le sommet de la feuille. Ce n'est que dans de rares espèces de Dicranoloma à feuilles plus ou moins obtuses qu'elle cesse un peu au-dessous du sommet. Souvent, dans sa partie supérieure, elle est canaliculée, ou bien, à la face dorsale, munie de dents aiguës sur $\mathbf{I}-2$ rangs.

Habituellement elle est un peu plus large à la base de la feuille et s'amincit légèrement et graduellement jusqu'au sommet. Toutefois, dans un certain nombre de Dicranoloma à nervure faible, celle-ci est à peine indiquée à l'extrême base et ne prend guère ses dimensions normales qu'un peu plus haut. D'ailleurs, la nervure est rarement homogène dans toute sa longueur et, outre les particularités signalées ci-dessus, il y a encore à constater la saillie dorsale plus fortement accusée dans le haut de la feuille que vers la base.

Pour se rendre compte de la structure de la nervure, il est donc nécessaire de l'examiner à des hauteurs différentes et, pour rendre les comparaisons possibles, il faut adopter 2 points conventionnels où devront toujours être faites les sections transversales, par exemple une coupe au $\mathrm{I} / 3$ inférieur et une autre au $\mathrm{I} / 3$ supérieur. Si l'on a soin 
de placer entre les lamelles de moelle de sureau 2 feuilles parallèlement, mais l'une la pointe en haut, l'autre la pointe en bas, le même coup de rasoir donnera à la fois les deux coupes.

La structure de la nervure examinée sur une section transversale comprend normalement les éléments suivants, en allant du centre à l'épiderme ventral ou dorsal :

$I^{\circ}$ Une couche de 2-I4 cellules à grande lumière (Deutern des Allemands- Eurycystes de Morin). Lorsque les eurycystes sont nombreux, ils forment une ligne courbée en arc, d'où l'expression : arc d'eurycystes.

$2^{\circ} \quad \mathrm{I}-3$ couches de cellules petites, à parois extrêmement épaissies, à lumen très faible, occupant l'espace compris entre l'arc central d'eurycystes et les surfaces ventrale et dorsale de la nervure.

Ces cellules stéréidiformes jouent le rôle d'éłéments de soutien et forment l'hypoderme.

$3^{\circ}$ I couche extérieure de cellules analogues à celles de l'hypoderme et constituant l'épiderme.

Eurycystes. - Les eurycystes, bien qu'à large lumen, ont les parois assez épaisses; leurs contours sont arrondis ou obscurément hexagones, plus ou moins réguliers et peuvent atteindre dans les Dicranoloma jusqu'à 18 et même 20 et 24 p.en diamètre. Il arrive aussi dans ce genre que, chez quelques espèces à eurycystes nombreux, ceux qui se trouvent aux 2 extrémités de l'arc, au nombre de 1 ou 2 de chaque côté, affleurent en épiderme sur la face ventrale.

Un peu plus petits dans les Leucoloma, les eurycystes affectent, en section transversale, une forme elliptique, le grand axe étant rarement perpendiculaire au plan de la feuille, presque toujours un peu oblique; en outre, dans l'espace compris entre chacune des extrémités de l'arc d'eurycystes et la naissance du limbe de la feuille, on trouve habituellement un groupe de 2-4 cellules à lumen moyen qui proviennent du dédoublement d'un eurycyste formant chacune des extrémités de l'arc. Dans les Leucoloma on ne remarque pas d'eurycystes terminaux affleurant en épiderme sur la face ventrale, comme dans quelques Dicranoloma, Dicranum et Campylopus.

Hypoderme. - Les cellules stéréidiformes de l'hypoderme qui servent d'éléments de soutien sont très petites et à lumen punctiforme, parfois même peu apparent. Lorsqu'on les examine sur une coupe trans 
versale et que la mise au point de l'objectif correspond à l'épaisseur moyenne d'une coupe préalablement éclaircie parl'eau de Javel ou le lacto-phénol d'Amann, on voit très distinctement apparaître le réseau très fin hexagonal des cellules de l'hypoderme. Si l'on relève très légèrement l'objectif de façon que la mise au point corresponde à la surface de la coupe, toute trace de réseau hexagonal disparaît et chaque cellule de l'hypoderme se présente sous une forme sphéroïdale, le lumen, quand il est visible, n'étant figuré que par un ostiole punctiforme. Tout l'hypoderme, surtout dans les Dicranoloma,ressemble donc à une série de petites sphères placées les unes à côté des autres et subtangentes. Comme, d'autre part, ces cellules considérées dans le sens longitudinal sont des polyèdres allongés, la forme sphéroïdale qu'elles affectent en coupe transversale semble due, soit à un jeu d'optique, soit au gonflement des parois épaisses et molles des cellules coupées dans la goutte d'eau du porte-objet, l'ostiole étant figuré par une petite dépression centrale.

Quelle que soit l'explication à donner de cette apparence sphéroïdale des cellules stéréidiformes de l'hypoderme, on la constate chez presque tous les Dicranoloma. Dans les Eudicranum, le D. scoparium par ex., dont la nervure est composée des mêmes éléments que celle de plusieurs Dicranoloma et semblablement disposés, les cellules hypodermiques plus ou moins régulièrement anguleuses dans leurs contours n'affectent pas la forme sphéroïdale d'une façon nette, l'épaisseur des parois sclérifiées est limitée et le lumen arrondi, petit, mais non punctiforme. Au point de vue des différenciations spécifiques, il peut être utile de signaler ces deux modes de tissu hypodermique qui se retrouvent chez beaucoup de Campylopus quoique la forme sphéroïdale (par ex. dans le $C$. crateris Besch. de La Réunion) soit la plus rare.

ÉPIDERME. - Dans les Leucoloma et Dicnemoloma l'épiderme est non ou très peu différencié de l'hypoderme.

Dans les Dicranoloma il est beaucoup plus variable, tantôt peu différencié, tantôt formé de cellules peu différenciées alternant avec des cellules plus amples, tantôt même comprenant des cellules à grande lumière, soit aux extrémités de l'arc à la face ventrale, soit, à la face dorsale, dans le type polycyclique, comme on le verra plus loin.

Tous les détails précédemment exposés concernant la.structure de la nervure dans nos 3 genres s'appliquent aux cas où ses éléments constitutifs sont au complet et normalement disposés, c'est-à-dire 2 couches épidermiques, l'une ventrale, l'autre dorsale, I-3 couches sur chaque 
face de cellules hypodermiques, enfin un arc central d'eurycystes. J'appellerai alors la nervure polystrosique (de otpwots, couche).

L'hypoderme manque dans de nombreuses espèces de Dicranoloma; l'épiderme se trouve directement en contact avec les eurycystes et la nervure ne comporte plus que 3 couches de cellules; je l'appellerai alors tristrosique.

Chez quelques espèces de Dicranoloma, il y a des intermédiaires entre ces deux modes de structure; l'hypoderme, au lieu de se présenter en couches continues sur chacune des deux faces, n'existe que sur l'une en manquant à l'autre, ou bien n'est plus indiqué que par quelques stéréides logées dans l'angle que forment les parois de deux eurycystes contigus; de là les expressions de subpolystrosique ou subtristrosique selon que ces formes de transition se rapprochent plus de l'un ou de l'autre des deux modes de structure définis ci-dessus.

Enfin, toujours dans le genre Dicranoloma, chez quelques espèces à nervure brusquement et fortement saillante sur la face dorsale, l'hypoderme, au lieu de s'étendre en couches continues entre l'épiderme et l'arc central d'eurycystes, est constitué par plusieurs (généralement 4) groupes de stéréides séparés par 2 lignes d'eurycystes ou plutôt pseudoeurycystes qui se détachent de l'arc central pour aboutir à l'épiderme de la face dorsale. J'appellerai alors la nervure polycyclique (de xuxios, groupe).

Il reste maintenant à examiner la structure dans chacun de nos trois genres.

Dicranoloma. - Très variée dans les Dicranoloma, la nervure offre des modifications qui peuvent se ramener à 4 types principaux. Quelques particularités de détail qui se présentent chez quelques espèces peuvent être utilisées au point de vue des distinctions spécifiques, mais n'altèrent pas sensiblement le caractère plus général de ces 4 types, auxquels on peut rattacher les nervures de toutes les espèces de Dicranoloma.

${ }^{\text {er }}$ type que j'appellerai toxoneuron (de rogov, arc). Nervure polystrosique, large (100-150 $\mu$ ), à section transversale en forme d'arc ou de croissant, peu incurvé sur la face ventrale, plus ou moins fortement convexe sur la face dorsale. Eurycystes centraux nombreux (8-14) Hypoderme ventral et dorsal formé de $2-3$ couches continues de stéréides. Epiderme comprenant I couche de cellules semblables à celles de l'hypoderme, nombreuses, jusqu'à 20-25, parfois un peu inégales. 
Ce type de nervure se rencontre dans les espèces suivantes:

D. Menziezii (Tayl.)............. Australie.

D. œdithecium (C. Müll.).......... Australie.

D. Kroneanum (C. Müll.).......... Australie.

D. suberectum (Hpe).............. Australie.

D. trichophyllum (Hpe)........... Australie, ile Chatam.

D. Armitii (C. Müll.) .............. Nouvelle Guinée.

D. bartramioides (Broth.) .......... Ile Lord Howe.

D. setosum (Hook f. et Wils.)........ Tasmanie.

D. grossialare (C. Müll.)........... Nouvelle Zélande.

D. dicranoïdes (C. Müll.) ........... Nouvelle Zélande.

D. calymperaceum (C. Müll.)........ Australie.

D. platyloma Besch.............. Nouvelle Calédonie.

D. formosum Besch.............. Nouvelle Calédonie.

D. perviride Par. et Broth........... Nouvelle Calédonie.

D. robustum (Hook.) .............. Terre de Feu.

D. Harioti Besch.................. Terre de Feu.

D. breviflagellare (C. Müll.)........ lles Sandwich, Hawai (1).

Ce type de nervure est celui qui se rapproche le plus de celui des Eudicranum. (Planche I, fig. a.)

$2^{\mathrm{e}}$ type que j'appellerai leptoneuron (de $\lambda \varepsilon \pi \tau \tau_{\varsigma}$, mince).

Nervure tristrosique mince, étroite $(20-50 \mu)$ à section transversale habituellement plate, débordant à peine le limbe, plus rarement un peu convexe sur la face dorsale. Eurycystes centraux très peu nombreux (deux). Hypoderme nul ou rudimentaire et alors réduit à de rares stéréides logées dans l'angle formé par 2 eurycystes contigus. Épiderme formé de cellules peu nombreuses (les dorsales 4-6), en contact direct avec les eurycystes.

C'est à ce type que se rattachent le plus grand nombre d'espèces de Dicranoloma. (Planche 1 , fig. $e, f$; planche 7 , fig. 4.)

D. Blumei (Nees.) ................ Java, Sumatra, Ceylon.

D. brevisetum (Doz. Molk.).......... Ceylon, Java.

D. arfakianum (C. Müll.) ........... Nouvelle Guinée.

D. novoguineense Broth. et Geh ...... Nouvelle Guinée.

D. Iævifolium Broth. et Geh.......... Nouvelle Guinée.

D. Deplanchei (Duby) ............. Nouvelle Calédonie.

D. Billardieri (Schwgr.)........... Australie, Chili, Pérou.

(1) Le classement de cette espèce dans le genre Dicranoloma reste douteux. Le margo foliaire, à peine et pas partout visible ainsi que l'aspect non sphéroïdal des cellules de l'hypoderme de la nervure, sont plutôt d'un Dicranum. 
D. angustinerve Mitt............. Australie.

D. austroscoparium (C. Müll.)....... Australie. Queensland.

D. Sullivani (C. Müll.)............. Australie.

D. austrocongestum (C. Müll.)....... Australie.

D. eucamptodontoïdes Broth. et Geh.. Tasmanie.

D. integerrimum Broth. et Geh....... Tasmanie.

D. Pungentella (C. Müll.)........... Tasmanie.

D. Weymouthii (C. Müll.)........... Tasmanie.

D. subsetosum (C. Müll.)............ Tasmanie, Australie.

D. Nelsoni (C. Müll.)............. Tasmanie.

D. confine (Hpe et C. Müll.)......... Australie.

D. subconfine Besch................ Ile d'Amsterdam.

D. pungens (Hook. fils et Wils)....... Terre de Feu.

D. Sandwicense (C. Müll.) ........... Iles Sandwich.

D. dichotomum (Brid.)............. Mascareignes.

D. scopareolum (C. Müll.) .......... Comores, Madagascar.

D. pycnoloma (C. Müll.)........... Madagascar.

D. patentifolium Ren. et Par......... Madagascar.

D. commutatum (Hpe)............. Afrique, Natal.

D. tabulare (C. Müll.)............. Afrique, Cap.

D. drepanocladium (C. Müll.) ....... Afrique, Kilimandjaro.

$3^{\circ}$ type que j'appellerai heteroneuron. Nervure tristrosique ou subpolystrosique, d'une largeur moyenne comprise entre 50 et $80 \mu$ selon les espèces; (cependant dans une forme de Sumatra voisine du D. assimile (Hpe) elle atteint $105 \mu$ ); section transversale plate ou un peu convexe sur la face dorsale, plus rarement en arc. Eurycystes centraux 4-5. Hypoderme nul ou peud éveloppé, interrompu ou bien encore n'existant que sur l'une des faces et ne comptant qu'une couche de cellules stéréidiformes. Epiderme parfois un peu différencié,en contact suivi ou interrompu avec les eurycystes selon que l'hypoderme manque ou est irrégulièrement figuré.

Comme on le voit, ce type est peu homogène et semble une transition entre les types toxoneuron et leptoneuron. S'il est difficile à définir dans ses traits généraux, en revanche il oftre par sa diversité même de précieuses indications pour les différenciations spécifiques. (Planche I, fig. $c, d$.)

Les espèces suivantes possèdent ce type de nervure.

D. subassimile Ren.............. Sumatra.

D. leucophyllum (Hpe)........... Java.

D. unıeııs Broth............... Australie. 

D. subpungens (Hpe)............. Australie.
D. australe (Besch.)............... Magellan.
D. pseudo australe (C. Müll.)........ Chili.
D. rigens (C. Müll.)............. Tasmanie.
D. austrocongestum (C. Müll.)....... Australie.
D. imponens (Montg)............. Patagonie.

$4^{e}$ type que j'appelerai cyrtoneuron (de xupros, convexe).

Nervure polycyclique d'une largeur moyenne (5o-8o $\mu$ ), brusquement et fortement saillante sur la face dorsale, à section transversale trapézoïdale, à angles bien marqués, plus rarement arrondis, incurvée à la face ventrale. Eurycystes centraux au nombre de 4, formant un arc très courbé. En outre, des deux eurycystes du centre de l'arc partent obliquement 2-3 eurycystes un peu moins grands que les premiers, quoique encore à grande lumière qui forment l'épiderme de la moitié dorsale de chacun des deux côtés latéraux du trapèze. Cette disposition des eurycystes figure ordinairement 2 lignes croisées en $x$, chacun des 4 angles de l'X étant occupé par des stéréides sur 2-3 rangs quiforment ainsi 4 groupes séparés, dont la rangée extérieure est l'épiderme. Celuici est donc, considéré dans son ensemble, constitué alternativement par des stéréides et des cellules à grande lumière.

Ce type est très caractérisé par la forme trapézoïdale de la section transversale, par les stéréides hypodermiques en groupes séparés et par la présence d'eurycystes en dehors de l'arc central. Quelquefois (rarement) l'hypoderme manque et les stéréides épidermiques sont directetement en contact avec l'arc central. La cellule à grande lumière qui forme le sommet de chacun des angles dorsaux du trapèze peut s'allonger en petite lamelle dentée, de sorte que le dos de la nervure, dans la subule, apparaît hérissé d'une double rangée de dents. (Planche I, fig. b.)

Cette forme de la nervure n'est généralement bien caractérisée qu'audessus du tiers inférieur et même parfois dans la moitié supérieure; plus près de la base, elle est toujours très convexe, mais arrondie sur la face dorsale et on n'y trouve pas toujours d'eurycystes, en dehors de ceux de l'arc central. (1)

(I) Dans le type toxoneuron, chez plusieurs espèces, la nervure passe au type cyrtoneuron vers le sommet de la feuille. 
Les espèces suivantes possèdent ce type de nervure:

D. assimile (Hpe),............. Java.

D. reflexifolium (C. Müll.).......... Java.

D. brevisetum (Doz. Molk.).......... Java, Ceylon.

D. Braunü (C. Müll.).............. Java.

D. Sumatranum Broth............ Sumatra.

D. reflexum (C. Müll.)............. Java.

D. polysetum (Hpe.)............. Australie.

D. dicarpum (Hornsch.)........... Australie.

D. Whiteleggei (C. Müll.).......... Australie.

D. lævifolium Broth. et Geh........ Nouvelle Guinée.

Leucoloma. - Dans les vrais Leucoloma la nervure est uniforme et ne se prête pas, comme celle des Dicranoloma, à la distinction de types différents. Elle est toujours polystrosique; sa largeur de 20 à $100 \mu$ est généralement en rapport avec les dimensions de la feuille, tandis que dans les Dicranoloma ce rapport existe peu. La section transversale est elliptique avec une courbure dorsale plus prononcée que la ventrale. Eurycystes centraux au nombre de 4, plus rarement 5-6, mais parfois partiellement et tangentiellement dédoublés de façon à se présenter en 2 couches centrales contiguës. La prolifération radiale de l'eurycyste de chacune des extrémités de l'arc se produit fréquemment dans les vrais Leucoloma. L'hypoderme est représenté par 2-3 couches de cellules stéréidiformes; l'épiderme reste peu distinct de l'hypoderme.

Dicnemoloma. - La structure de la nervure dans les espèces peu nombreuses examinées est analogue à celle des vrais Leucoloma. (Planche I, fig. $g$.) 


\section{CHAPITRE II}

\section{CLASSIFIGATION}

Il est naturel de chercher une base de classification dans l'accord entre les caractères histologiques et les caractères morphologiques; mais les uns et les autres ne suivent pas toujours une marche parallele et, en outre, la structure anatomique des différentes parties du système végétatif offre des variations souvent indépendantes les unes des autres, soit d'espèce à espèce, soit de groupe à groupe, de sorte qu'on est obligé de spéculer sur la plus grande somme d'analogies. Les caractères histologiques qui ont le plus de généralité servent à distinguer les groupes plus élevés (genres et ensuite sous-genres), tandis que ceux qui présentent le plus de particularités sont utilisés pour les différenciations spécifiques. Telle est la marche générale que j'ai suivie ; mais c'est en réalité dans le choix des caractères histologiques que résident les principales difficultés d'une classification.

\section{G E N RES}

Si nous examinons l'ensemble des espèces réunies plus ou moins anciennement au genre Leucoloma et possédant en commun la présence d'un margo blanchâtre bordant les feuilles, nous distinguons immédiatement par l'inspection du tissu foliaire deux grandes séries. Dans la première (Dicranoloma) ce tissu est uniforme transversalement et il y a toujours homogénéité entre les cellules juxtacostales et les cellules juxtamarginales prises au même niveau. Dans la deuxième série (Leucoloma et Dicnemoloma) il y a différenciation du tissu foliaire en cellules internes chlorophylleuses et cellules membraneuses, celles-ci, occupant les ailes basilaires, dans les Leucoloma, tandis que ces mêmes ailes basilaires sont constituées dans les Dicnemoloma par les cellules internes chlorophylleuses. 
Ces différences dans la disposition des éléments anatomiques du limbe de la feuille sont générales et ne souffrent aucune exception. Elles sufisent à elles seules à distinguer nettement entre eux ces trois genres. En dehors de ces données histologiques qui sont les plus importantes pour séparer les 3 genres, il en est d'autres fournies par les diverses parties du système végétatif. Nous les suivrons dans chaque genre.

Dans le genre Dicranoloma, la tige est pourvue d'un faisceau axile. La nervure est très variée dans sa structure et oscille entre deux types 'extrêmes, l'un très simple ne comprenant qu'un petit nombre d'eurycystes $(2-4)$ qui se trouvent directement en contact avec les cellules épidermiques peu nombreuses aussi, sans l'interposition d'un hypoderme, l'autre plus compliqué comprenant jusqu'à 14-í 6 eurycystes séparés, sur. les faces ventrale et dorsale, de l'épiderme par une ou plusieurs couches d'éléments de soutien hypodermiques, composés de cellules à petite lumière plus ou moins stéréidiformes.

Dans les vrais Leucoloma et Dicnemoloma, la tige est dépourvue de faisceau axile. La structure de la nervure est uniforme et constituée par un arc médian d'eurycystes en petit nombre, généralement 4 , plus rarement 5-6, entourés sur les deux faces de $2-3$ couches de cellules à petite lumière, stéréidiformes et d'un épiderme très peu distinct de l'hypoderme. Si dans quelques espèces ou quelques individus, il y a dédoublement tangentiel et partiel d'ailleurs des eurycystes, c'est un fait accidentel qui n'infirme en rien la régularité générale de la structure de la nervure.

$\mathrm{Si}$, à ces données histologiques on ajoute les caractères morphologiques, on arrive aux diagnoses suivantes de nos 3 genres.

Gen. I. Dicranoloma Ren. - Faciès des Dicrana scoparia. Tissu foliaire non différencié, dicranoïde; papilles dorsales nulles ou peu saillantes. Structure de la nervure très hétérogène selon les espèces, comprenant de 2 à I 4 - I 6 eurycystes, hypoderme souvent nul. Tige pourvue d'un faisceau axile sclérifié. Coiffe nettement cucullée dépassant beaucoup la base de l'opercule. Capsule généralement arquée (dressée symétrique dans peu d'espèces). Dents du péristome (16) fendues jusqu'au milieu ou au delà. Inflorescence dioïque ou monoïque.

Gen. II. Leucoloma Brid. - Faciès varié dicranoïde ou spécial. Tissu foliaire différencié composé de tissu chlorophyllien juxtacostal (cellules internes) et de tissu membraneux (cellules intermédiaires), ce dernier 
occupant toujours les ailes basilaires au-dessus des oreillettes; papilles dorsales souvent très saillantes, parfois bifides, rarement indistinctes. Structure de la nervure uniforme, variant très peu selon les espèces, comprenant ordinairement 4 eurycystes, plus rarement 5-6; épiderme peu distinct de l'hypoderme, celui-ci constitué par des éléments sclérifiés stéréidiformes en 2-3 couches. Tige et base du pédicelle dépourvues de faisceau axile sclérifié. Coiffe petite dépassant à peine la base de l'opercule, plurilobée à la base ou fendue d'un seul côté, ordinairement papilleuse au sommet. Capsule dressée symétrique. Dents du péristome (ı) divisées jusqu'au milieu ou au delà. Inflorescence dioïque.

Gen. IIl. Dienemoloma Ren. - Faciès spécial non dicranoïde. Tissu foliaire différencié. Tissu chlorophyllien (cellules internes) occupant presque toute la feuille et descendant le long des marges jusqu'aux oreillettes, le tissu membraneux ne s'étendant que sur un espace restreint juxtacostal à la base de la feuille; papilles dorsales généralement très saillantes. Structure de la nervure uniforme et analogue à celle des Leucoloma. Tige et base du pédicelle dépourvues de faisceau axile sclérifié. Coiffe cucullée, capsule arquée. Dents du péristome 16 , fendues audessus du milieu ou seulement au sommet. Inflorescence dioïque (I).

Ces 3 genres sont incontestablement distincts les uns des autres; toutefois on pourra être tenté de réunir le genre Dicranoloma, comme sous-genre, au genre Dicranum dont il ne diffère que par les feuilles munies aux bords d'un margo hyalin analogue à celui des Leucoloma. On ne saurait songer à séparer génériquement les espèces de Mnium à feuilles marginées de celles qui ne possèdent.pas de margo, parce que

(r) Lorsque j'ai publié, en $\mathbf{1} 897$, dans le Prodrome de la Flore bryologique de Madagascar le sous-genre Dicnemoloma Ren., j'ignorais l'existence dans l'herbier de Hampe du nom de Dicnemonella appliqué par ce savant bryologue à une espèce unique d'Australie: Leucoloma Sieberi, sans qu'aucune diagnose du nouveau genre ait été publiée. Il est fort probable que ce genre Dicnemonella de Hampe correspond à mon genre Dicnemoloma; il est probable aussi (autant du moins qu'on en peut juger par le nom) que Hampe s'est basé pour établir son genre sur la fissure peu profonde des dents péristomiales; mais n'ayant eu en vue qu'une espèce, la seule connue à l'époque, il lui aurait été difficile de tabler sur la disposition des éléments anatomiques du limbe de la feuille. J'ai pu au contraire, grâce à la découverte de plusieurs espèces analogues, conclure à la généralité (qui ne s'est d'ailleurs pas démentie dans les ro espèces connues actuellement) du caractère histologique tiré du tissu foliaire et qui m'a conduit, plus encore que la fissure peu profonde des dents péristomiales, insuffisante à elle seule, à fonder le genre Dicnemoloma. Pour ces diverses raisons, je crois devoir maintenir ce genre décrit, comme sous-genre, en 1897. D'ailleurs le fait que le genre Dicnemonella Hpe est resté à l'état de nomen nudum, tranche définitivement la question. 
ces dernières sont en minorité et, d'ailleurs, n'habitent pas une zone déterminée, mais se trouvent mêlées aux autres dans des localités analogues.

Il en est tout autrement des Dicranoloma qui habitent la région tropicale australe et ses extensions vers le sud, tandis que les vrais Dicranum (subg. Dicranum Limprt) qui leur correspondent comme caractères généraux n'y sont représentés que par un petit nombre d'espèces localisées; la plupart, dans les parties élevées des Andes. Les autres Dicranum de l'hémisphère sud appartiennent presque tous aux sous-genres Chorisodontium et Leiodicranum; qui sont disséminés, le premier dans les hautes montagnes des Andes et de l'Afrique australe, le second dans les régions tempérées ou froides du Chili et des Terres Magellaniques.

Que cette tendance des cellules marginales à s'épaissir en se différenciant et à former un margo dans les Dicranoloma soit le résultat d'actions climatériques accumulées qui se traduisent par une activité non interrompue de la végétation, c'est une hypothèse à examiner et d'après laquelle on pourrait considérer les Dicranolona comme des Dicranum modifiés. De fait, en dehors de leur margo caractéristique, ils reproduisent non seulement les traits principaux de ces derniers, mais encore des variations analogues dans leurs formes.

On ne peut guère tenter de vérifier cette hypothèse qu'en tenant compte des données biologiques, dont la distribution géographique des espèces est comme la résultante,et j'ai cherché à reconnaître si la richesse en espèces et l'accentuation dans celles-ci du caractère principal des Dicranoloma, c'est-à-dire,la largeur du margo, correspond au climat tropical le plus accusé.

Il est nécessaire d'entrer ici dans quelques détails et de suivre la distribution des Dicranoloma dans l'étendue de la zone tropicale.

Dans l'archipel des Sandwich, sous le tropique du Cancer, on a signalé 3 espèces dont 2 surtout sont si mal caractérisées génériquement, à margo si peu visible qu'elles ne semblent guère pouvoir être détachées du genre Dicranum.

Il faut arriver aux abords de l'Équateur pour entrer dans la véritable zone des Dicranoloma.

Ceylan: 2 espèces à margo étroit, mais bien visible.

Java et Sumatra: 12 espèces dont 3 très faiblement, 4 étroitement, 2 assez largement marginées; 3 espèces non examinées.

Nouvelle Guinée : 5 espèces, toutes à margo très étroit. 
Australie (littoral de l'Est) : 20 espèces dont 8 très faiblement, 4 assez largement, 4 largement ou très largement marginées; 4 espèces non examinées.

Tasmanie: ro espèces dont 3 très faiblement, 3 étroitement, 4 assez largement marginées.

Nouvelle Calédonie: 6 espèces, dont 2 largement, 4 très largement marginées.

Nouvelle Zélande: Ile d'Amsterdam, île Chatam, île de Lord Howe: Io espèces dont 2 très faiblement, 3 étroitement, 3 assez largement marginées ; 2 espèces non examinées.

Amérique : Aucune espèce signalée dans la zone intertropicale de l'Amérique (pas plus d'ailleurs que de l'A sie continentale, si ce n'est une seule au Khasia). C'est seulement dans le Chili méridional à Valdivia $\left(40^{\circ}\right.$ lat. austr.) qu'on voit apparaître les premières espèces au nombre de 3, dont l'une commune avec l'Australie, assez largement marginée, et les deux autres à margo étroit ou très étroit. Quelques espèces s'avancent même jusqu'au détroit de Magellan et à la Terre de Feu (lat. $55^{\circ}$ ). On en compte i 3, dont 4 étroitement et 9 assez largement marginées. Dans cette région du Cap Horn, flces Dicranoloma sont accompagnés d'une douzaine de Dicranum (subg. Chorisodontium et Leiodicranum). A la Nouvelle Géorgie (lat. $56^{\circ}$ ) on ne trouve plus que des Dicranum, les Dicranoloma ont disparu.

Afrique: Une seule espèce au Kilimandjaro (4 ${ }^{\circ}$ lat. Sud), une au Natal, une au Cap, toutes à margo très étroit.

Madagascar, Mascareignes et Comores : 3 espèces très étroitement marginées.

De cet exposé se dégagent les conclusions suivantes qui ne confirment pas l'hypothèse émise plus haut.

I $^{\circ}$ Le nombre des espèces de Dicranoloma et l'accentuation de leur caractère générique principal ne paraît pas lié au cachet tropical plus ou moins accusé du climat.

$2^{\circ}$ Le genre est tropical en ce sens qu'il exige une somme de chalcur et d'humidité ne descendant pas au-dessous du minimum nécessaire pour qu'en aucune saison la végétation ne soit interrompue; mais il est surtout austral et manque à la plus grande partie de la zone tropicale boréale, ou ne s'y trouve guère qu'au voisinage de l'Équateur, tandis qu'il s'avance vers le Sud bien au delà du tropique du Capricorne, par exemple jusqu'à la Terre de Feu oủ d'ailleurs le suivent quelques représentants de genres tropicaux tels que: Macromitrium, Rhyngogonium, Harissonia, Mniadelphus, Hypopterygium. 
$3^{\circ}$ Le fait qui prime tous les autres dans la distribution des Dicranoloma est l'existence d'un centre de végétation comprenant les îles de la Sonde, Celèbes, Philippines, le littoral oriental de l'Australie, la Nouvelle Calédonie, la Tasmanie, la Nouvelle Zélande et le Chili méridional. C'est là que se trouvent le plus grand nombre d'espèces et, dans ce domaine, c'est en Nouvelle Calédonie et sur le littoral australien du Queensland qui lui fait face, que croissent les espèces à caractère générique le plus marqué.

Chez quelques espèces de Dicranoloma le margo foliaire devient si étroit et si mal défini qu'on a peine à l'apercevoir. Tantôt il se réduit à 2 séries de cellules ou même à une seule, tantôt il est visible seulement dans certaines feuilles ou dans certaines parties de la feuille; ailleurs enfin, les cellules médianes se rétrécissent progressivement peu à peu de façon que la marge de la feuille paraît bien un peu scarieuse; mais sans qu'on puisse définir nettement le point où le vrai margo commence. Dans ces cas le classement de la plante reste un peu indécis entre les Dicranoloma et les Dicranum. C'est ainsi que quelques espèces placées par M. Brotherus parmi les Dicranoloma me laissent du doute sur leur attribution à ce genre (notamment 3 espèces des îles Sandwich qui me semblent plutôt être des Dicranum, les D. Armitii C. M., D. novoguineense Broth. et Geh., D. nematosum Broth. et D. lavifolium Broth. et Geh. de la Nouvelle Guinée, D. Graeffeanum C. M. de Samoa D. nigricaule Angstr. des Terres Magellaniques). De cette même région les $D$. peruncinatum Dus., D. subimponens Card. et D. Falklandicum Card. me paraissent aussi devoir être considérés comme des Dicranum.

De nouvelles observations sur de plus nombreux échantillons me semblent nécessaires pour fixer la place générique de ces espèces. Mais, quand même il resterait de l'indécision pour quelques espèces, on ne pourrait invoquer ce fait contre le maintien du genre Dicranoloma. La même absence de margo se rencontre aussi chez quelques vrais Leucoloma. C'est d'ailleurs une banalité de rappeler que de nombreux genres, même des mieux établis, n'ont pas toujoursdes limites absolument précises, sans que l'on songe pour cela à les supprimer. Dans le cas qui nous occupe, les espèces à caractère générique douteux sont du reste en infime minorité.

Il est possible que les Dicranoloma et les Dicranum dérivent d'un type ancestral commun; mais comme on l'a vu, on ne saurait reconnaî tre une action modificatrice du climat agissant encore aujourd'hui et il faut chercher l'explication de la dispersion actuelle des Dicranoloma 
dans une distribution antérieure ou première apparition dont le mode nous est inconnu. De fait, les deux genres s'excluent presque complètement et nous voyons même, dans l'hémisphère austral, les Dicranoloma manquer dans le vaste bassin de l'Amazone qui possède au plus haut degré le climat tropical chaud et humide, tandis que des espèces assez nombreuses s'accommodent du littoral assez sec de l'Australie et quelques-unes de la température peu élevée de la Terre de Feu, compensée il est vrai par le cachet maritime très accusé du climat, la grande humidité, le ciel nuageux et les hivers peu rigoureux.

\section{SOUS-GENRES}

Dicranoloma. - La distinction de sous-genres dans les Dicranoloma soulève une difficulté qui n'est pas particulière à ce genre, mais se retrouve dans d'autres genres où les variations de la structure histologique de la nervure ne concordent pas avec celles des caractères morphologiques. C'est ainsi, par exemple, que les $D$. Blumei de Java et D. dichotomum des Mascareignes, qui ont le même type de nervure, sont aux pôles opposés comme caractères carpologiques. Si on prend ces derniers comme base pour distinguer des sous-genres, il n'y a aucun compte à tenir de l'histologie de la nervure.

Nous sommes donc conduits, pour rechercher s'il y a lieu d'établir des coupes subgénériques, à examiner séparément les caractères morphologiques et les caractères histologiques.

Pour les premiers, en se basant sur la forme et la direction de la capsule et le renflement du col capsulaire, on peut établir les 2 sousgenres suivants :

Subg. I. Scoparioïdium: - Capsule à col lisse ou à peine renflé, arquée ou plus rarement dressée-symétrique.

Subg. II. Oncophorioïdium. - Capsule toujours arquée, à col muni d'une apophyse saillante.

Les espèces de Dicranoloma se classent en nombre à peu près égal dans chacun de ces sous-genres, entre lesquels quelques espèces à col capsulaire muni d'une apophyse peu saillante restent un peu indécises. On pourrait aussi envisager l'état lisse ou papilleux des dents péristomiales; mais je n'ai pu les observer dans un nombre suffisant d'espèces pour pouvoir tirer une conclusion de cet examen.

Au point de vue des caractères histologiques, il y a lieu d'examiner le tissu du limbe de la feuille et la structure de la nervure.

Le tissu du limbe offre 3 types principaux. 
I 0 Type scoparioïde, c'est-à-dire analogue à celui du Dicranum scoparium et composé de cellules linéaires, allongées, à parois épaisses, poreuses, dépourvues de papilles, généralement d'aspect scarieux. Ce type est celui de la grande majorité des espèces de Dicranoloma.

$2^{\circ}$ Dans une douzaine d'espèces plus ou moins affines, dont le D. Menziezii est la plus connue, la base seule de la feuille brusquement dilatée et brièvement demi-vaginante est formée de cellules linéaires allongées. Au-dessus de cette base, les cellules deviennent courtes, carrées ou subhexagones, chlorophylleuses et plus ou moins papilleuses sur la face dorsale, non poreuses.

$3^{\circ}$ Enfin se présente chez le $D$. dicarpum Brid. d'Australie un type de tissu foliaire constituant une exception unique, presque contraire à la définition même du genre. La feuille est marquée de 2 plis profonds longitudinaux; l'espace compris entre la nervure et le pli est formé de cellules assez amples, larges et courtes, les juxtacostales carrées ou subhexagones, s'allongeant peu à peu vers le pli où elles deviennent brièvement rectangulaires ou oblongues-sublinéaires, 4-6 fois aussi longues que larges, tandis que la partie comprise entre le pli et le bord de la feuille est formée de cellules prosenchymateuses étroites et très allongées, I 5-2o fois aussi longues que larges, qui dessinent une bande de tissu serré très distincte de la bande juxtacostale.

Cette différenciation du tissu pourrait faire croire à un vrai Leucoloma; mais tous les autres caractères sont contraires à cette interprétation: l'inflorescence monoïque du D. dicarpum, tandis que tous les Leucoloma sont dioïques, la capsule incurvée, la coiffe nettement en capuchon et descendant jusque vers le milieu de la capsule, enfin la structure de la nervure et de la tige, celle-ci pourvue d'un faisceau axile sclérifié. En outre, le tissu de la bande juxtacostale est beaucoup plus lâche et moins obscur que les cellules internes des vrais Leucoloma. On n'en doit pas moins considérer cette espèce australienne, si remarquable sous ce rapport comme une forme établissant la liaison entre les Dicranoloma et les vrais Leucoloma. Le D. Whiteleggei C. Müll. de la Nouvelle Galles du Sud a la même structure du tissu foliaire que le D. dicarpum; mais ses caractèrès spécifiques sont si légers relativement à ce dernier, qu'il n'en est vraisemblablement qu'une race régionale.

Comme on l'a vu, il n'y a pas de concordance entre les caractères morphologiques et ceux qu'on pourrait tirer des diverses modifications de la structure de la nervure. Y a-t-il plus de liaison entre ces derniers et les différents types de tissu foliaire énumérés plus haut? C'est ce qu'il reste à examiner. 
$1^{\circ}$ La grande majorité des espèces de Dicranoloma (près de 50) ont un tissu foliaire scoparioïde et, parmi elles, on en compte 27 dont la nervure est du type Leptoneuron, 8 du type Heteroneuron, 6 du type Cyrtoneuron et 7 du type Toxoneuron. Malgre le nombre important d'espèces possédant à la fois un tissu scoparioide et une nervure du type Leptoneuron, on ne peut en former un sous-genre distinct, une vingtaine d'autres espèces à tissu scoparioïde ayant une structure différente de la nervure. En outre, 2 espèces $D$. austrocongestum C. Müll. et D. serratum Broth. ont un tissu foliaire ambigu, non nettement scoparioïde, parenchymateux, à cellules brièvement rectangulaires ou rhombées, sans qu'on puisse l'assimiler exactement à celui des espèces du groupe du D. Menziezii.

$2^{\circ}$ Ce groupe du $D$. Menziezii, bien caractérisé non seulement par son tissu foliaire, mais encore par la grande analogie de port des espèces qui lecomposent, paraît très naturel. La nervure y est toujours du type Toxoneuron; mais ce même type de nervure se retrouvant dans 7 espèces dont le tissu foliaire est nettement scoparioïde, il n'est pas possible de faire concourir ensemble les deux caractères histologiques pour la distinction d'un sous-genre.

$3^{\circ}$ Dans le $D$. dicarpum dont le tissu foliaire est si spécial, la nervure est du type Cyrtoneuron. Ce même type se retrouvant dans 6 espèces à tissu scoparioïde, ici encore on ne peut utiliser conjointement les deux caractères.

De toutes ces constatations il résulte que les caractères histologiques se prêtent mal à l'établissement de coupes subgénériques dans le genre Dicranoloma, par suite de leur manque de concordance, soit entre eux, soit relativement aux caractères morphologiques. Il est donc préférable de se baser sur ces derniers et de conserver les 2 sous-genres Scopariödium et Oncophoriö̈dium tels qu'ils ont été indiqués plus haut, encore qu'ils ne soient fondés que sur un seul caractère un peu artificiel.

Leucoloma. - La distinction des sous-genres dans les vrais Leucoloma est infiniment plus facile que dans les Dicranoloma et se trace pour ainsi dire d'elle-même. La diversité des éléments anatomiques du limbe de la feuille fournit de bons caractères suffisants par eux-mêmes et qui ont, de plus, l'avantage de n'être pas en désaccord avec les caractères carpologiques, ces derniers d'ailleurs d'une importance moyenne. La structure de la nervure, par suite de son uniformité, ne peut jouer aucun rôle dans la formation des groupes. 
C'est dans la disposition des éléments anatomiques du limbe et surtout la place occupée par les cellules chlorophylleuses internes qu'il faut chercher une base pour établir les coupes subgénériques. Nous arrivons ainsi aux 3 sous-genres suivants :

Subg. I. Tæniodictyon Ren. - Faciès spécial ou vaguement dicranoïde. Cellules internes formant de chaque côté de la nervure deux bandes parallèles régulières, nettement séparées, sur les côtés, du tissu membraneux et se prolongeant jusqu'à la base de la feuille ou à peu près. Papilles généralement peu saillantes. Oreillettes planes formées de cellules scalariformes. Pédicelle court dépassant peu la longueur de la capsule, celle-ci presque toujours renflée ovale-oblongue ou subglobuleuse.

Subg. II. Sphenodictyon Ren. - Faciès spécial non dicranoïde. Feuilles généralement bossuées sur le dos, souvent ridées en travers, papilleuses sur les deux faces, à papilles denses, bifides. Cellules internes formant une masse obscure bien séparée sur les côtés du tissu membraneux voisin et se terminant en coin à une distance notable de la base qui est entièrement formée de tissu membraneux. Oreillettes planes composées de cellules scalariformes. Fructification connue dans une seule espèce et analogue à celle du subg. Tæniodictyon.

Subg. III. Syneratodictyon Ren. - Faciès généralement dicranoïde, rarement spécial. Cellules internes ne formant pas de bandes juxtacostales, nettement délimitées sur les côtés et passant par des transitions insensibles soit sur les côtés, soit vers la base, au tissu membraneux qui occupe toute la base. Papilles petites ou presque nulles dans quelques sections, très proéminentes et bifides dans d'autres. Oreillettes excavées formées de cellules non scalariformes. Pédicelle allongé ; capsule étroite subcylindrique.

Dicnemoloma. - Les Dicnemoloma comptent un trop petit nombre d'espèces pour qu'il y ait lieu d'y rechercher des groupes subgénériques.

\section{SEC.TIONS}

A mesure que nous descendons la série hiérarchique des groupes, nous devons faire intervenir des caractères secondaires, employés auparavant seulement à titre accessoire pour les groupements plus élevés. 
Ce sont maintenant surtout des caractères morphologiques qu'on peut utiliser: port des plantes et mode de végétation, forme et direction des feuilles, etc. Toutefois les caractères histologiques trouvent encore leur emploi pour délimiter certaines sections ; c'est notamment la forme des cellules du limbe et leurs dimensions, la densité et la saillie plus ou moins forte des papilles qui se développent à la face dorsale, plus rarement sur les deux faces et qui rendent le tissu plus ou moins obscur.

Dicranoloma. - Il y a lieu de distinguer 2 sections dans le sousgenre scoparioïdium :

$1^{0}$ Orthotheca. Capsule symétrique, dressée.

$2^{\circ}$ Campylotneca. Capsule arquée plus ou moins inclinée.

Cette dernière section comprend la grande majorité des espèces du sous-genre. La première ne compte que 7 espèces qui, toutes, appartiennent à Java, quelques-unes communes avec Ceylon, Sumatra et la Nouvelle Guinée: D. Blumei (Nees), D. brevisetum (D. M.), D. Braunii (C. Mull.), D. dives (C. Mull.), D. brachypelma (C. Mull.), D. reflexum (C. Mull.), D. reflexifolinm (C. Mull.).

On devrait, à la rigueur, admettre dans les Orthotheca le $D$. Armitii (C. Mull.) de la Nouvelle Guinée dont la capsule très courte, elliptique, renflée est symétrique ou presque symétrique. Mais, d'autre part, cette espèce a tant d'affinités avec celles du groupe du D. Menziezii (H. et T.) qu'on ne saurait l'en séparer. C'est là l'inconvénient d'une classification basée sur un seul caractère. En réalité, le genre Dicranoloma se prête mal à des coupes subgénériques et à un sectionnement par suite du manque d'accord des différents caractères. En revanche, il est possible d'isoler des groupes d'espèces ayant entre elles des affinités évidentes, sans pour cela établir un sectionnement régulier qui impliquerait le classement de toutes les espèces du genre. Ces séries qui ne comprennent que les espèces pouvant être rapprochées et ne portant pas de nom hiérarchique autre que celui de "groupes", servent surtout pour la construction des tableaux synoptiques destinés à faciliter les déterminations. Sous ce rapport leur utilité est incontestable.

C'est ainsi que ce groupe du $D$. Menziezii se distingue immédiatement par l'aspect plumeux des touffes, par les feuilles élargies à la base et rétrécies rapidement en une longue subule presque entièrement occupée par la nervure, celle-ci toujours large et épaisse, polystrosique, du type toxoneuron, enfin par les cellules moyennes et supérieures courtes, non poreuses, finement papilleuses sur la face dorsale. 
Cet ensemble de caractères suffirait largement à la distinction d'une section; mais, parmi les nombreuses autres espèces du genre, on ne trouverait pas un autre groupe aussi bien défini. On reconnaîtrait seulement des espèces reliées par un caractère commun, mais non par un ensemble de traits distinctifs.

Le groupe du $D$. Menziezii comprend $\delta$ espèces, en majeure partie australiennes, 1 de la Nouvelle Guinée, 1 de l'île Chatam, 1 de l'île de Lord Howe.

D. Menziezii (Tayl.), D. Kroneanum (C. Müll.), D. suberectum (Hpe), $D$. cedithecium (C. Müll.), D. trichophyllum (Hpe), D. bartramioïdes Broth., D. setosum (Hook. Wils.), D. Armitii (C. Müll.).

Leucoloma. - Trois sections à établir dans le sous-genre Tænodictyon:

Sect. I. Euvittata Ren. - Cellules internes chlorophylleuses distinctes, formant une lame régulière descendant jusqu'à la base de la feuille ou à peu près. Papilles peu saillantes et seulement à la face dorsale. Cellules des oreillettes à parois peu épaissies. Faciès non dicranoïde. Touffes d'un vert plus ou moins jaunâtre ou roux. Feuilles souvent incurvées-crispulées à l'état sec, ovales-oblongues, plus rarement lancéolées, brièvement cuspidées, plus rarement subulées.

Sect. II. Subvittata Ren. - Cellules internes chlorophylleuses distinctes formant une lame qui ne descend pas tout à fait jusqu'à la base de la feuille. Papilles peu saillantes et seulement sur la face dorsale. Cellules des oreillettes à parois peu épaissies. Faciès dicranoïde.Feuilles plus ou moins secondes, non crispulées à l'état sec, oblongues-lancéolées ou lancéolées, assez ou très longuement subulées, à nervure généralement excurrente.

Obs. - Cette section établit une transition entre le sous-genre Tænodictyon et quelques sections du sous-genre Syncratodictyon, notamment les sections Semivittata Broth. et Dicranoïdea Besch. A l'état fertile, l'examen de la longueur du pédicelle ne permet pas de confusion; mais, à l'état stérile, certaines espèces paraissent un peu indécises entre les deux sous-genres. Dans la section Subvittata, les cellules internes suprabasilaires s'allongent peu à peu, perdent leur chlorophylle et leurs papilles, et forment, à la base, un tissu scarieux ayant quelque analogie avec celui des ailes membraneuses: mais les 
cellules juxtacostales basilaires quoique plus allongées que les moyennes et supérieures conservent toujours une forme oblongue qui les différencie de celles des ailes membraneuses; tandis que dans le sous-genre Syncratodictyon, le tissu basilaire reste membraneux jusqu'à la nervure. Dans les cas douteux, qui sont d'ailleurs l'exception, il faut examiner le passage brusque ou insensible des cellules internes chlorophylleuses au tissu membraneux vers le milieu de la feuille et, d'autre part, le tissu basilaire juxtacostal. L'un au moins de ces deux caractères suffira pour décider entre les 2 sous-genres.

Sect. III. Obscura Ren. - Cellules internes non ou peu distinctes, formant une lame obscure, opaque, descendant jusqu'à la base de la feuille. Papilles très denses, habituellement sur les 2 faces. Cellules des oreillettes à parois épaissies. Faciès non dicranoïde. Touffes d'un vert olivâtre ou brun. Feuilles un peu épaisses.

Le sous-genre Sphenodictyon ne comprend jusqu'à présent que six espèces, dont 2 de Madagascar très affines: L. tuberculosum Ren. et L. Levieri Ren. exigent une mention particulière parce que le caractère distinctif du sous-genre ne s'y montre pas toujours bien nettement. La lame chlorophylleuse interne se termine bien au-dessus de la base; mais elle est souvent peu amincie à son extrémité inférieure et imparfaitement cunéiforme. En outre, cette lame, au lieu d'être densément papilleuse sur les deux faces et rendue par cela même obscure, présente à la face dorsale des papilles espacées très élevées bi-trifides. En revanche, les feuilles bossuées sur le dos et fortement marquées de rides transversales qui correspondent à des lignes de papilles offrent une telle analogie avec les espèces du sous-genre Sphenodictyon que ces 2 espèces sont mieux placées dans ce sous-genre que dans le subg. Trniodictyon (section Obscura) où l'on serait tenté de les caser.

Sous-genre Syncratodictyon. - On peut distinguer dans ce sousgenre 7 sections dont 4 très naturelles et 3 ne comprenant chacune qu'une seule espèce qu'il serait d'ailleurs difficile de classer dans aucune des autres sections.

Sect. I. Cæspitulosa Besch. in Florule Réunion. - Forme des tapis plus ou moins étendus, peu cohérents. Tige très courte. Feuilles d'un vert obscur, brièvement subulées, munies tout au sommet de dents 
fortes, digitées, crispées à l'état sec, fortement papilleuses, à papilles dorsales très saillantes bifides, les apicales plus ou moins courbées en crochet.

Obs. - Dans cette section les cellules internes descendent très bas le long de la nervure dans la partie élargie de la feuille oú elles passent insensiblement au tissu basilaire, celui-ci parenchymateux, composé de cellules rectangulaires peu allongées, à parois fermes, mais à lumen assez large.

Sect. II. Holomitrioidea Ren. - Port rappelant en petit celui des Holomitrium. Tige courte dressée, rameaux fastigiés très densement feuillés, épais. Feuilles petites largement ovales à la base, puis rapidement contractées en un acumen court, presque toujours cassé, crispées à l'état sec.

Une seule espèce habitant Madagascar.

Sect. III. Pseudo cæspitulosa Ren. - Plantes humbles formant des tapis souvent étendus sur les écorces, d'un vert pâle ou jaunâtre. Tige très courte. Feuilles ténues, étroites, lâchement flexueuses, parfois falciformes à l'état sec, mais non franchement crispulées. Papilles dorsales saillantes, les supérieures ordinairement en crochet.

Obs. - Cette section est intermédiaire entre les Caspitulosa et les Albescentia. Des premiers elle se rapproche par le gazonnement étendu, la couleur verte et la brièveté des tiges; des seconds par les feuilles plus ténues, flexueuses ou subfalciformes, non crispulées, de façon à reproduire comme une réduction de cette section Albescentia.

Sect. IV. Albescentia Besch. - Plantes formant des touffes d'un vert pâle se décolorant souvent et prenant une couleur blanc-jaunâtre. Feuilles habituellement subsecondes-falciformes, plus ou moins ondulées aux bords, ce qui fait ressortir le brillant du margo hyalin ordinairement large et se prolongeant presque jusqu'au sommet par une seule série de cellules. Cellules internes grosses $(3-5 \mu)$ relativement à la grandeur de la feuille, unipapilleuses. Papilles dorsales saillantes, aiguës ou bifides, les supérieures en crochet.

Sect. V. Semivittata Broth. - Cellules internes formant dans la moitié supérieure de la feuille une lame nettement séparée sur les côtés du tissu membraneux voisin et se fondant peu à peu dès le milieu ou le 
tiers inférieur dans le tissu de la base qui est nettement membraneux. Oreillettes planes à cellules scalariformes. Port dicranoïde.

Obs. - La séparation brusque sur les côtés de la lame interne et du tissu membraneux, ainsi que les oreillettes planes à cellules articuleses, rapprochent beaucoup cette section, qui n'est encore représentée que par une seule?espèce, de la section Subvittata du sous-genre Taniodiclyon; mais le tissu basilaire nettement membraneux sur toute sa largeur et surtout le pédicelle allongé doivent faire incontestablement classer dans le sous-genre Syncratodictyon cette plante (L. secundifolium Mitt. de l'Afrique équatoriale occidentale), qui par le port ressemble aux espèces de la section Dicranoïdea. Comme dans cette dernière section, les papilles dorsales sont peu saillantes.

Sect. VI. Dicranoidea Besch. - Plantes assez robustes ou robustes d'un vert franc ou foncé ou roux. Port dicranoïde. Feuilles presque toujours falciformes longuement subulées-sétacées. Papilles dorsales fines, peu saillantes, parfois presque nulles. Oreillettes amples excavées.

Sect. VII. Dichelymoïdea (Besch. ex parte) Ren. emend. - Port des formes grêles de Dichelyma. Tige filiforme molle très allongée. Feuilles espacées subdistiques étalées dressées. Papilles dorsales à peine saillantes. Oreillettes amples, planes, formées de cellules articulées.

Obs, - L'unique espèce $: L$. dichelymö̈des que comprend jusqu'à présent cette section a, à peu près, la même structure d'oreillettes que le $L$. secundifolium Mitt. de la section Semivittata; mais son port est très spécial et elle trouverait difficilement place dans les sections déjà établies.

Je conserve à cette section le non de Dichelymoïdea que Bescherelle a employé dans sa Florule de la Réunion; mais je l'entends dans un sens restreint au seul $L$. dichelymoïdes, Bescherelle ayant compris dans sa section des espèces appartenant aux sous-genres Taniodictyon et Sphenodictyon.

\section{ES PE C ES}

Il serait superflu de reproduire ici les idées le plus généralement reçues sur la conception de l'espèce. En dehors d'un certain nombre de types nettement délimités et peu variables, une foule d'espèces doivent être comprises, moins comme une entité absolue que comme un groupe 
de formes. On manque d'un criterium certain pour juger la valeur de l'espèce et l'idée que chacun s'en fait comporte toujours un certain degré de subjectivité.

Ce qu'il est permis de répéter, c'est que l'examen de matériaux exotiques représentés, comme c'est le cas habituel, par des échantillons d'herbier souvent incomplets, conduit à distinguer des espèces nouvelles dont l'autonomie ne peut être affirmée tout d'abord parce qu'on ne connaît pas encore l'étendue de leurs variations. A tous les botanistes s'occupant de semblables travaux, il est arrivé de fonder avec doute une espèce nouvelle d'après des caractères qui semblent légers et de voir plus tard cette espèce s'affirmer par la fixité de ces caractères reconnus dans de nouveaux matériaux. Dans un sens contraire, telle espèce nouvelle qui paraissait bonne au début devient d'une valeur douteuse lorsque, par l'apport de nouveaux documents, on découvre des formes de transition entre cette prétendue espèce et une espèce déjà connue.

$\mathrm{La}$ fondation de quelques mauvaises espèces est un inconvénient inévitable dans tout premier travail et il ne faudrait même pas que cette considération empêchât de donner un nom à une forme qui semble nouvelle; il suffit seulement de la décrire exactement et de bien indiquer ses affinités. L'erreur commencerait là où l'on s'obstinerait à la maintenir comme valable, alors que de nouveaux échantillons démontrent qu'elle ne dépasse pas les limites des variations d'une espèce connue. Ce n'est que par étapes successives qu'on arrive à fixer la valeur réelle d'une espèce, et, encore, faudrait-il qu'on ait pu suivre dans la nature, dans les lieux où elle est abondante, son degré de polymorphisme et les formes qu'elle affecte. Dans tout ceci, d'ailleurs, si le principe est banal, l'application à en faire dans une juste mesure est souvent chose difficile.

Pour les espèces de $2^{\mathrm{e}}$ ordre et les races régionales, on doit tenir compte des conditions biologiques auxquelles la distribution géographique est intimement liée, des facteurs climatériques, surtout en ce qui concerne la répartition des pluies et de l'humidité, des limites plus ou moins nettes des régions habitées par ces races, enfin de la présence ou de l'absence au milieu d'elles du type dont elles semblent dériver. Souvent l'examen de ces considérations jette un jour inattendu sur des questions obscures, quand il s'agit de déterminer le degré de valeur spécifique à attribuer à telle ou telle forme affine.

Le manque de concordance entre les caractères morphologiques et les données histologiques qui a quelquefois, comme on l'a vu plus 
haut, entravé la formation de groupes plus ou moins élevés, n'a plus d'importance quand il s'agit de délimiter des espèces. Ici, tous doivent être employés pour permettre d'arriver à la plus grande somme de différences possible et les caractères histologiques être considérés non seulement dans leurs traits généraux, mais, s'il en est besoin, dans des particularités de détail qui aident souvent à différencier les espèces.

Lorsqu'un sectionnement satisfaisant a été établi, il importe d'élaguer les caractères de section des descriptions d'espèces qui deviennent ainsi plus courtes, plus claires et permettent de saisir plus vite les différences spécifiques. Avec beaucoup de raison on indique aujourd'hui les mesures micrométriques des divers organes. Si les dimensions ne constituent pas par elles-mêmes un caractère de premier ordre, on ne peut nier qu'en Cryptogamie elles ne rendent de signalés services pour les déterminations.

Bien que la méthode générale suivie dans l'étude des divers organes des Mousses, au point de vue taxinomique, soit applicable à tous les genres, néanmoins les mêmes organes ne sont pas également variables dans tous; dans ceux surtout qui comptent de nombreuses espèces, il y a à faire un choix de caractères morphologiques et histologiques que l'on doit suivre dans toutes les espèces. On peut recommander les suivants pour aider aux distinctions spécifiques dans nos 3 genres.

Dicranoloma. - Direction et forme des feuilles, longueur et finesse de la subule, denticulation des marges. Oreillettes plus ou moins développées. Tissu scoparioïde ou non, plus ou moins dense et poreux. Margo hyalin plus ou moins large et continu, nettement ou vaguement séparé du tissu voisin, sa largeur exprimée en $\mu$ et nombre de séries de cellules dont il est formé.

Largeur de la nervure vers la base (exprimée en $\mu$ ), son excurrence plus ou moins longue, lisse ou munie sur la face dorsale supérieure de I-2 rangées de dents ; forme de la section transversale et structure anatomique. C'est surtout dans le type Heteroneuron qu'il faut reconnaître les particularités servant aux distinctions spécifiques.

Quelques exemples, mieux qu'une règle générale, d'ailleurs difficile à formuler, feront ressortir le parti à tirer sous ce rapport de la structure de la nervure.

Dicranoloma subassimile Ren. Section transversale plate. Nervure tristrosique, hypoderme nul, 4 grands eurycystes. Epiderme ventral formé de 8-ı o cellules stéréidiformes subégales; épiderme dorsal formé 
de 5 cellules inégales, les 3 cellules correspondant aux points de tangence des eurycystes plus grandes et séparées par des groupes de 3 cellules plus petites égales. (Planche I, fig. c.)

Dicranoloma australe Besch. Section transversale comme dans le D. subassimile Ren., mais 5 eurycystes et hypoderme ventral figuré par une couche de stéréides; hypoderme dorsal nul.

Dicranoloma subpungens (Hpe). Section transversale en forme d'arc ; tristrosique; hypoderme nul; 6 eurycystes dont les deux de chaque extrémité affleurant en épiderme ventral. Cellules épidermiques à moyenne lumière, les ventrales 4-5, les dorsales I 2-I4. ( $\mathrm{Pl}$. I, fig. d.)

Ces différences sont très suffisantes pour aider à la distinction de ces deux espèces. En revanche, dans les nombreuses espèces possédant une nervure du type Leptoneuron, il n'y a à utiliser que de bien faibles. notes, par exemple :

Dicranoloma Blumei (Nees.). Section transversale absolument plate ne débordant pas le limbe de la feuille même à la face dorsale; 2 eurycystes ; 4 cellules épidermiques ventrales, 4 dorsales. (Pl. vir, fig. 4.)

Dicranoloma dichotomum (Brid.). Section transversale légèrement convexe sur la face dorsale; 2 eurycystes; cellules épidermiques ventrales 4 , les dorsales 7. (Pl. I, fig. e.)

Dicranoloma scopareolum (C. Müll.). Section transversale presque plate quoique débordant le limbe à la face dorsale. Eurycystes 2 ; cellules épidermiques ventrales 3 , les dorsales 5 (Pl. I, fig. $f$.)

Dicranoloma patentifolium Ren. Par. Section transversale assez. fortement convexe sur la face dorsale, le reste comme dans le D. scopareolum. (Pl. v, fig. g.)

L'écueil à éviter serait de donner trop de généralité à quelques différences de détail qui pourraient être particulières à l'échantillon examiné plutôt qu'à l'espèce. Plusieurs coupes seront donc nécessaires. Il faut reconnaître d'ailleurs quel'histologie de la nervure, en dehors des 4 types décrits dans un chapitre précédent et qui se distinguent facilement, ne se prête pas toujours aux différenciations spécifiques. Ses variations suivant une marche indépendante, on ne peut guère l'utiliser pour éta- 
blir une filiation ni même un sectionnement, tandis qu'en revanche elle permet ordinairement la distinction de groupes spéciaux d'espéces.

Cette observation n'est pas applicable seulement aux genres dont il est question ici. C'est ainsi que les coupes subgénériques établies dans le genre Campylopus, en prenant pour base l'histologie de la nervure, offrent le grave inconvénient de séparer des espèces évidemment très affines et d'en réunir d'autres non moins incontestablement disparates.

Leucoloma. - Direction, grandeur et forme des feuilles ; longueur et finesse de la subule, denticulation des marges, leur enroulement. Oreillettes plus ou moins développées, planes ou excavées. Développement du tissu chlorophyllien (cellules internes) et délimitation de l'espace qu'il occupe relativement au tissu membraneux, obscur ou distinct par suite de la densité plus ou moins grande des papilles dont les cellules sont couvertes, dimensions (en $\mu$ ) de celles-ci. Papilles plus ou moins saillantes, arrondies, pédicellées, bifides ou en crochet, proéminentes sur la face dorsale seulement ou sur les 2 faces. Tissu basilaire membraneux serré et comme scarieux ou plus lâche, parenchymateux composé de cellules rectangulaires courtes ou allongées. Margo hyalin plus ou moins large (largeur en $\mu$ et nombre des séries de cellules dont il se compose), distinctement ou mal séparé du tissu membraneux contigu ou même à peu près nul.

Largeur de la nervure à la base (en $\mu$ ), son excurrence plus ou moins longue, dos lisse ou denté vers le sommet. La structure histologique, à cause de son uniformité, ne fournit presque pas de caractères spécifiques. La prolifération radiale des 2 cellules formant les extrémités de l'arc d'eurycystes, quoique très fréquente, correspond à une tendance habituelle plutôt qu'elle n'est propre à telle ou telle espèce. Le nombre d'eurycystes est généralement constant (4-6) et on ne pourrait citer que peu d'espêces, par exemple L. sinuosum Brid. des îles austro-africaines dont le nombre d'eurycystes s'élève jusqu'à 8-io.

Les organes de reproduction ne peuvent être que rarement utilisés à cause de la stérilité habituelle des échantillons. La longueur du pédicelle sert surtout à la distinction des sous-genres. Au point de vue spécifique, il y a lieu d'examiner la forme de la capsule, cylindrique, ou plus ou moins renflée ou même subglobuleuse, contractée ou non sous l'orifice après la chute de l'opercule, les dimensions des dents péristomiales, la grosseur des spores.

Les caractères spécifiques sur lesquels doit se porter principalement 
l'attention du bryologue étant connus, il s'agit, pour l'étude, de les fixer dans la mémoire et comme ils sont, pour la plupart, anatomiques, le souvenir en est promptement effacé et, surtout, dans les genres à nombreuses espèces, comme c'est ici le cas, il est indispensable d'en conserver la trace par le dessin. L'usage de la chambre claire doit être fréquent, continuel même. Cette méthode, qui paraît au premier abord compliquer l'étude, la simplifie au contraire considérablement. Il n'est point besoin pour cela de posséder un talent spécial de dessinateur. Il suffit de reproduire sur une feuille volante séparée un simple schema indiquant les caractères principaux de chaque espèce en ayant soin d'employer toujours les mêmes grossissements pour figurer les mêmes organes.

On acquiert assez vite une habitude suffisante pour ces reproductions. Les échantillons originaux de l'herbier doivent d'abord être dessinés de cette façon. Sans cette précaution ils seraient promptement détériorés ou même anéantis par les comparaisons multiples qu'on est obligé de faire pour les déterminations. Il faudra en outre conserver une bonne préparation de quelques feuilles de chaque espèce type.

Pour les échantillons à déterminer, à moins de cas particuliers où l'on pourrait soupçonner immédiatement le nom de l'espèce, il est préférable d'en dessiner à la chambre claire un schema qui sera comparé à ceux du type et des autres échantillons rapportés précédemment à telle espèce. Grâce au système des feuilles volantes la comparaison de ces schemas devient facile parce qu'ils peuvent être embrassés d'un seul coup d'œil.

La comparaison de l'image virtuelle reçue par l'œil dans le microscope avec le schema de l'espèce type ne donnerait pas un aussi bon résultat, au moins sous le rapport de l'exactitude des dimensions micrométriques. On pourra facilement s'en convaincre en adaptant au microscope un micromètre oculaire et en traçant, au jugé, sur une feuille de papier, une ligne représentant, telle qu'on la voit dans le microscope, la longueur d'un nombre quelconque de divisions du micromètre. Si ensuite on trace, à l'aide de la chambre claire, la même ligne, dans les mêmes conditions, on verra que cette dernière qui représente la longueur exacte est presque toujours beaucoup plus grande (presque du double) que la ligne tracée au jugé. J'ai vérifié l'exactitude de cette indication que je dois à M.Orzeszko. On ne peut donc utilement comparer qu'une image virtuelle à une autre image virtuelle, ou un schema à un schema. 
Il sera bon aussi de joindre aux échantillons étudiés et classés dans l'herbier une préparation de plusieurs feuilles. On peut employer pour cela un procédé que j'ai vu appliquer par un de mes correspondants américains et qui me paraît pratique. On découpe dans du carton mince 2 lames ayant la forme et les dimensions du porte-objet ordinaire et on pratique au centre de chacune de ces lames une ouverture ou fenêtre d'une dimension convenable, de façon que les fenêtres des 2 lames se correspondent exactement. On prépare quelques feuilles ou autres organes entre 2 lamelles de mica, selon la méthode ordinaire; on place cette préparation sur la fenêtre de l'une des lames de carton et on recouvre avec l'autre lame que l'on colle sur la première. La préparation est renfermée avec l'échantillon dans son enveloppe et lorsqu'on veut l'utiliser, il suffit de la placer sur la table du microscope, comme on ferait d'un porte-objet. L'emploi du carton et du mica a pour but d'éviter la fragilité du verre. 


\section{CHAPITRE III}

\section{DISTRIBUTION GÉOGRAPHIQUE}

D'une manière générale les Leucoloma sont des plantes tropicales; mais chacun de nos 3 genres a, dans sa dispersion, des allures particulières qu'il est nécessaire d'exposer séparément.

Dicranoloma. - Il a été parlé déjà de cette question dans le chapitre relatif à la classification; aussi suffira-t-il de résumer brièvement les conclusions.

Ce genre est plus austral que tropical; car il dépasse de plus de 20 degrés vers le sud le tropique du Capricorne, tandis que, dans l'hémisphère boréal, il se maintient aux environs de l'équateur. (Le classement dans les Dicranoloma des 3 espèces signalées aux îles Sandwich me semble douteux).

C'est dans une distribution antérieure plutôt que dans les conditions climatériques qu'il faut chercher l'explication de ces faits de dispersion et on ne saurait imaginer quelle cause climatérique pourrait éloigner les Dicranoloma, genre tropical, du vaste bassin de l'Amazone chaud et humide, tandis qu'on voit des espèces s'avancer vers le sud jusqu'aux régions froides de la Terre de Feu ou au littoral relativement sec de l'Australie et du Cap.

Le centre de végétation le plus riche en espèces comprend les îles de la Sonde, le littoral oriental de l'Australie, la Nouvelle Calédonie, la Tasmanie, la Nouvelle Zélande et le Chili méridional.

L'Afrique est pauvre en espèces, le continent asiatique encore davantage (une seule espèce au Khasia) ainsi que la zone intertropicale de l'Amérique. Ce n'est que dans le Chili méridional à Valdivia (40 lat. 
austr.) qu'on voit apparaître les Dicranoloma dont quelques espèces s'avancent sur le versant du Pacifique jusqu'au détroit de Magellan et à la Terre de Feu (lat. $55^{\circ}$ ), tandis qu'on n'en trouve plus a la Nouvelle Géorgie (lat. $55^{\circ}$ ).

La répartition des Dicranoloma par continents est la suivante :

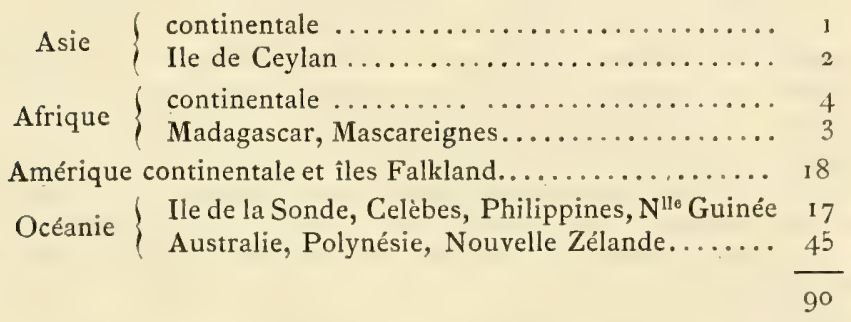

En réalité le Domaine occupé par les Dicranoloma comprend surtout les terres baignées par le Pacifique austral. Le bassin de l'Océan Indien ne comprend que peu d'espèces et l'on n'en connaît pas encore de l'Océan Atlantique.

Si les vastes lacunes qu'on remarque dans l'aire de la dispersion des Dicranoloma ne s'expliquent pas par la seule considération des conditions climatériques et doivent être attribuées surtout à un mode de distribution antérieur, il ne s'en suit nullement qu'ils n'aient pas, comme d'ailleurs la plupart des plantes, si ce n'est toutes, des exigences particulières relativement au climat. On remarquera tout d'abord que, sans être des espèces absolument littorales, ils ne s'écartent des côtes que dans des limites relativement restreintes et manquent complètement à l'intérieur des grands continents. En dehors des îles, on ne peut citer, comme habitats, que le Khasia en Asie, non loin du golfe du Bengale, en Afrique que le Kilimandjaro, le Natal et le Cap, en Amérique que la côte méridionale du Chili et, en Australie que le littoral oriental de ce continent.

Toutes les espèces recherchent donc un climat essentiellement maritime, même avec un contingent de pluies médiocre comme le littoral australien et le Cap ou une température peu élevée comme celle du Chili méridional et du Fuegia, pourvu que, ni d'une part la sècheresse, ni de l'autre, le froid ne soient assez accentués pour causer une interruption dans la période de végétation. 
Une autre remarque à faire est que les Dicranoloma semblent bien des espèces de la zône des forêts de montagne. Dans les régions très chaudes on ne les trouve qu'à partir d'une certaine altitude, par exemple 50o-6oo mètres dans les îles austro-africaines, 800 mètres à Java où une espèce est signalée jusqu'à 2000 mètres, au Kilimandjaro où le D. drepanophyllum (C. Müll.) est indiqué de 1800 à 3000 mètres.

On pourrait croire que ces exigences d'altitude sont le motif pour lequel les Dicranoloma manquent aux vastes plaines de l'Amazone; mais Richard Spruce, le célèbre explorateur de cette région, a poussé ses investigations jusque vers le versant oriental des Andes dans le bassin supérieur du Rio Negro et de l'Uaupès etles riches collections qu'il en a rapportées ne comprennent aucune espèce du genre. Même absence dans les chaînes du Brésil qui ont cependant une altitude suffisante.

En ce qui concerne la distribution des sections, il faut citer la section Orthotheca dont les 7 espèces sont localisées dans les îles de la Sonde, toutes à Java, quelques-unes communes avec Ceylan, Sumatra et la Nouvelle Guinée.

Le groupe spécial du D. Menzięii Tayl. comprend 8 espèces en majeure partie australiennes, I de la Nouvelle Guinée, I de l'île Chatam, I de l'île de Lord Howe.

Enfin, les espèces remarquables par leur très large margo sont particulières à la Nouvelle Calédonie et au littoral australien du Queensland qui lui fait face.

La plupart des espèces de Dicranoloma sont endémiques et restent cantonnées sinon dans la même île, du moins dans des groupes d'îles peu distantes où elles sont représentées, soit par des formes identiques, soit par des espèces extrêmement voisines. C'est ainsi que $D$. dichotomum des Mascareignes est remplacé à Madagascar et aux Comores par D. scopareolum et D. patentifolium Ren. et Par. et au Cap par D.commutatum et $D$. tabulare; puis, on le retrouve dans le continent africain au Kilimandjaro.

On ne peut citer qu'un petit nombre d'espèces: $D$. Billardieri, D. Menziezii, D. robustum Hook f. et Wils, D. setosum Hook. Wils. qui aient une aire de dispersion plus étendue délimitée par le Chili méridional, la terre de Feu, le littoral oriental de l'Australie, la Nouvelle Zélande, l'île Campbell.

Les échanges se font ordinairement entre Ceylan et les îles de la Sonde, d'autre part entre l'Australie, la Tasmanie et la Nouvelle Zélande. En revanche, on ne saisit pas de relations entre les terres du Pacifique 
et celles de l'Océan Indien. La Nouvelle Calédonie conserve, comme sous d'autres rapports, un puissant cachet d'individualité.

Leucoloma. - Les vrais Leucoloma sont un genre plus nettement tropical que les Dicranoloma. Un assez grand nombre d'espèces se montrent dès le tropique du Cancer et, dans l'hémisphère austral, les plus avancées ne dépassent guère que de 10 à 12 degrés le tropique du Capricorne (Cap de Bonne Espérance). Si dans ce dernier hémisphère les espèces sont plus nombreuses que dans l'hémisphère boréal, ce fait est dû au plus grand morcellement des terres qui multiplie les zones littorales ou sublittorales à climat maritime accusé, tandis que les vastes surfaces continentales plus ou moins sèches ou désertiques de l'hémisphère tropical boréal ne conviennent pas aux vrais Leucoloma qui sont des plantes hygrotropicales.

Quelques espèces croissent aux plus basses altitudes, par exemple sur les sables du littoral à Madagascar, quoique la plupart habitent les forêts sur l'humus et les troncs; d'autres s'élèvent assez haut dans les montagnes : 2000 mètres à Java, 1600 mètres au Cameroun, 2000 mètres au Kilimandjaro, ı 300-1 400 mètres à Madagascar.

De même que les Dicranoloma, les Leucoloma occupent surtout les îles et une lisière littorale parfois assez large des continents, sur les versants soumis à l'influence des vents marins humides et suffisamment arrosés par les pluies; de plus, comme on l'a vu, il leur faut un plus fort coéfficient de chaleur. Mais, la part de leurs exigences climatériques ainsi faite, celle qui revient à une distribution antérieure indépendante du climat se manifeste par des différences considérables dans la position des centres de végétation, à ce point que la richesse relative en espèces des deux genres est presque toujours en sens inverse.

C'est ainsi que, dans le bassin du Pacifique, les îles de la Sonde, la Nouvelle Guinée, l'Australie et la Polynésie ne fournissent que peu d'espèces. En revanche, le bassin de l'Atlantique, à peu près dépourvu de Dicranoloma,est habité par de nombreux Leucoloma dès le tropique du Cancer, par exemple aux Antilles, puis au Vénézuela, dans le bassin de l'Amazone, au Brésil et, en Afrique par quelques espèces à la Guinée française, au Cameroun, à Saint-Thomé et au Gabon ( $\mathrm{I}$ )

L'Océan Indien l'emporte sur les deux autres par l'abondance des

(1) Je n'ai pas reconnu une seule espèce de Leucoloma dans les envois successifs que j'ai reçus du bassin intérieur du Congo par les explorateurs belges qui ont poussé jusqu'au delà de Stanley Falls. 
Leucoloma. Il ne peut être question du littoral occidental du continent australien qui ne peut recevoir les pluies apportées par l'alizé du SudEst et reste désertique. Le Siam, l'Inde méridionale, les îles Andaman fournissent quelques espèces. A Ceylan se trouve par exception un mélange de Dicranoloma et de Leucoloma, ces derniers un peu plus nombreux.

L'Afrique orientale au Kilimandjaro, dans l'Usambara, à Zanzibar, au Natal et au Cap comprend un certain nombre d'espèces; mais ce sont surtout les îles austro-africaines de l'Océan Indien : Seychelles, Mascareignes, Comores et Madagascar qui constituent le centre de végétation le plus important. Madagascar surtout est d'une incomparable richesse et originalité de formes.

Le tableau suivant résume ces indications:

Asie et Malaisie....................... I 7

Afrique et îles......................... 64

Amérique et Antilles...................... 26

Océanie et Polynésie.................... 7

I 44

La division des Leucoloma en sous-genres et en sections met en relief des groupes très naturels et bien distincts d'espèces affines dont il est d'un grand intérêt de suivre la dispersion.

Tout d'abord, il faut signaler le sous-genre Sphenodictron si spécial comme endémique dans les îles austro-africaines: Madagascar, Mascareignes et Seychelles. Jusqu'à présent, il n'a pas encore été constaté aux Comores. Sur les 6 espèces qu'il comprend, 5 croissent à Madagascar dont I commune avec les Mascareignes et I espèce (peut-être une simple race) habite les Seychelles.

Dans le sous-genre Teniodictyon, la section Obscura qui confine d'ailleurs sous quelques rapports au sous-genre Sphenodictyon est également presque endémique à Madagascar et à la Réunion où elle comprend 3 espèces. En dehors de ces îles, on retrouve, par une exception singulière, une autre espèce de cette section au Mexique $(L$. cryptocarpum (C. Müll.).

La section Euvittata est richement représentée à Madagascar (r o espèces), puis à La Réunion et aux Comores (4 espèces), pauvrement sur le continent Africain où l'on ne connaît encore que 2 espèces, l'une de l'Usambara, l'autre de l'île de Saint-Thomé. On en compte I espèce 
à Java (commune avec Hong Kong), I à Ceylan, I au Vénézuela, 3 au Brésil et I au Costa Rica.

La section Subvitlata est, au contraire, jusqu'à ce jour inconnue à Madagascar et aux Mascareignes; elle ne comprend qu'une seule espèce aux Comores. Les espèces de cette section sont réparties de la façon suivante: Inde méridionale 2 espèces, Ceylan 2, Australie (Queensland), Polynésie (Raratonga) et Nouvelle Guinée 6; Antilles 4, Vénézuela I, Brésil 1. Cette section manque à l'Afrique, au moins jusqu'à présent.

Dans le sous-genre Srncratodictyon, la section Caspitulosa comprend 8 espèces dont 4 propres à Madagascar, aux Mascareignes et aux Comores, 3 à l'Inde méridionale et aux îles Andaman et I aux Petites Antilles.

La section Albescentia est presque exclusivement propre à l'Afrique orientale et aux île austro-africaines de l'Océan indien. Ces dernières fournissent 8 espèces (Madagascar, Mascareignes et Comores); le continent 2 dans le Zanzibar et 4 au Cap ou Natal. L'Amérique n'en compte que 2.

La section Pseudocaspitulosa, qui est voisine de la précédente, est également bien représentée à Madagascar (3 espèces) et compte I espèce dans le continent africain; les 3 autres croissent dans les Antilles et l'Amérique centrale (Costa Rica, Vénézuela, Equateur, Pérou, Brésil).

La section Dicranoïdea, la plus répandue peut-être, n'a pas de centre de végétation bien déterminé. Elle-paraît toutefois manquer à peu près aux îles de la Sonde et à l'Australie et on pourrait presque ajouter aux terres du Pacifique (seulement I espèce à Quito, I espèce à Tahiti). Les îles austro-africaines comptent 6 espèces, presque toutes fréquentes ou très fréquentes dans leurs localités, le continent Africain 4 espèces (Usambara, Kilimandjaro, Cameroun); Ceylan I espèce, les Antilles 4 espèces, le Brésil 2.

Enfin 3 sections monotypes doivent être signalées comme forcément très localisées :

$1^{\circ}$ Section semivittata : I espèce $L$. secundifolium Mitt., île de SaintThomé (Gabon).

$2^{\circ}$ Section Holomitrioïdea: I espèce $L$. cuneifolium C. M. qui, sous quelques rapports, se rapproche de la section Cicspitulosa, Madagascar.

$3^{\circ}$ Section Dichelymö̈dea: I espèce $L$. dichelymö̈des assez commune à Madagascar, ne ressemblant à aucune autre et qu'on ne pourrait comparer que de loin au $L$. secundifolium.

Il ressort de cet exposé que les îles austro-africaines de l'Océan 
Indien constituent un puissant foyer de production remarquabl, none seulementpar la présence de tous les groupes et par l'endémisme de quelques-uns, mais encore par l'originalité de quelques types dont on ne retrouve pas ailleurs l'équivalent et aussi par l'abondante diffusion de quelques espèces (par ex. du L. Rutenbergii à Madagascar).

Les Antilles et le golfe du Mexique avec une quinzaine d'espèces forment aussi un centre d'une importance notable qui ne peut toutefois être comparé au précédent et dont la plupart des espèces appartiennent aux sections Dicranoïdea et Subvittata qui sont les plus répandues. Il est de même des 5 espèces de l'Amazone et du Brésil, de sorte que toute l'Amérique, malgré ses 26 espèces, ne possède que des types assez. uniformes et sans grande originalité.

Le genre Leucoloma est à peine représenté sur le versant austroaméricain du Pacifique.

Dionemoloma. - Ce genre est surtout australien (6 espèces en Australie, 2 à Java et I aux Celèbes). En dehors de ce centre, on trouve, par une anomalie singulière, une seule espèce au Brésil.

\section{ÉNUMÉRATION SYSTÉMATIQUE DES LEUCOLOMA}

Sous-genre Tæniodictyon Ren.

Section I. Euvitrata Ren.

L. semibruneum C. Müll. Brésil

L. costaricense Ren. Costa Rica

L. triforme Mitt. Brésil

L. nitens Mitt. Ceylan

L. tenerum Mitt. Ceylan

L. molle Mitt. Java

L. biplicatum Hpe. Brésil

L. mollisimum Mitt. Vénézuela

L. gracilescens Broth. Afr. (St-Thomé)

L. Holstii Broth. Afrique (Usambara)

L. Woodii Reh. Transwaal

L. bifidum Brid. Madagas., Mascar.
L. subbifidum Ren. Madagascar

L. Lepervanchei Besch. La Réunion

L. squarrosulum C. Müll. Madagascar

L. subbiplicatum Ren. Card. Madag.

L. chrysobasilare C. Müll. Comores

L. procerum Ren. Comores

L. subchrysobasilare C. Müll. Madag.

L. Mafatense Ren. La Réunion.

L. silvaticum Ren. Madagascar

L. leptocladum Ren. Madagascar

Section II. Subvitrata Ren.

L. Cheesemani Ren. Polynésie (Arch. Cook)

L. tenuifolium Mitt. Samoa 
L. oceanicum C. Müll. Samoa

L. Baueræ C.Mül. Aust. (Queensland)

L. subintegrum Broth. Australie (Queensland)

L. Kanakense Broth. et Par. Nouvelle Calédonie

L. novæ Guineæ C. Müll. Nlle Guinée

L. serrulatum Brid. Martinique

L. caldense C. Müll. Brésil

L. schwaneckeanum $\mathrm{Hpe}$. Antilles

L. Catharinæ C. Müll. Brésil

L. ecaudatum C. Müll. Vénézuela

L. lucinerve Mitt. Ceylan

L. insigne C. Müll. Ceylan

L. pallidicaule C. Müll. Philippines

L. Riedlei Besch. Antilles, St-Thomas

L. Comoræ Ren. Comores

Section III. Obscura Ren.

L. cryptocarpum C.Müll. Mexique

L. Sanctæ Mariæ Besch. Madagascar

L. Thuretii Besch. Madagascar

L. cinclidotioïdes Besch. Madagascar

L. subtuberculosum Ren. Madagascar

Sous-genre Sphenodictyon Ren.

L. Seychellense Besch. Seychelles

L. Crepini Ren. Card. Madagascar, Mascareignes.

L. Grandidieri Ren. Card. Madagas.

L. Talazaccii Ren. Card. Madagascar

L. tuberculosum Ren. Madagascar

L. Levieri Ren. Madagascar

Sous-genre Syncratodictyon Ren.

Section I. Cespitulosa Besch.

L. tortellum Mitt. Brésil

L. Walkeri Broth. Inde mérid. Coorg
L. crispofalcatum Broth. Inde, Moulmein

L. Taylori (Schwgr.) Mitt. Inde, Népal

L. sarcotrichum C. Mül. Iles Andaman

L. Dussianum Besch. Guadeloupe

L. cespitulans C. Müll. Comores

L. amblyacron C. Müll. Maurice

L. subcespitulans Besch. La Réunion

L. cirrosulum Ren. Madagascar

Section II. Holomitrioïdea Ren.

L. cuneifolium Hpe. Madagascar

Section III. Pseudocespitulosa Ren.

L. subimmarginatum C. Müll. Costa Rica

L. Ambreanum Ren. Card. Madagas.

L. delicatulum Ren. Madagascar

L. convolutaceum Ren. Madagascar

Section IV. Albescentia Besch.

? L. asperrimum C. Müll. Vénézuela

L. syrrhopodontoïdes Broth. Pondoland

L. Zanzibarense Besch. Zanzibar

L.scabricuspis Broth. Afrique, Usag.

L. Rehmanni C. Müll. Transwaal

L. Sprengelianum C. Müll. Cap

L. Zeyheri C. Müll. Cap

L. Eckloni Lor. Cap

L. sinuosulum C. Müll. La Réunion

L. candidulum C. Müll. Maurice

L. Isleanum Besch. Seychelles, Comores

L. Brotheri Ren. Madagascar

L. persecundum C. Müll. Mascareignes, Madagascar

L. albocinctum Ren. Card. Madag.

L. pumilum C. Müll. Madagascar 
? L. aduncum Hpe. Brésil

? L. Leichardtii Hpe. Australie

Section V. Semivittata Broth.

L. secundifolium Mitt. Afrique occid.

Section VI. Dicranoidea Besch.

L. limbatulum Besch. Tahiti

L. Therioti Ren. Card. Brésil

L. procumbens Mitt. Pérou

L. Moseni Broth. Brésil

L. macrodon Hook. Equateur

L. portoricense C. Müll. Porto Rico

L. Renauldii Broth. Inde mérid.

L. malabarense Besch. Inde, Malabar

(Coorg)
Malabar

L. amœnevirens Mitt. Ceylan

L. Birmense C. Müll. Tenasserim

L. Beautei Besch. Siam

L. Siamense Broth. Siam

L. subalbulum Ren. Guadeloupe (I)

L. longicapillare C. Müll. Jamaïque

L. albulum Sull. Antilles

L. Crügerianum C. Müll. Antilles

L. Mariei Besch. Guadeloupe

L. Volkensii Broth. Afr., Kilimandjaro

L. subsecundifoliun Broth. Afrique, Usambara
L. terricolum Broth. Afr. Usambara

L. Cameruniae C. Müll. Cameroun

L. Normandi Broth. Par. Afr. Fouta Djallon

L. pygmæum Par. Afr. Guinée franç.

L. sinuosum Brid. Mascareignes, Afr.

L. fuscifolium Besch. Mascareignes, Madagascar

L. Dubyanum Besch. Maurice

L. Boivini Besch. Comores, Madag.

L. Garnieri Ren. Par. Madagascar

L. Rutenbergii C. Müll. Madagascar

L. capillifolium Ren. Madagascar (Sainte-Marie)

Sect. VII. Dichelymoidea (Besch.) Ren.

L. dichelymoïdes C. Müll. Madagas.

\section{Genre Dicnemoloma Ren.}

D. Sieberi Hornsch. Australie

D. clavinerve C. Müll. Australie

D. Fraseri Mitt. Australie

D. imbricatum Broth. Geh. Australie

D. strictipilum C. Müll. Australie

D. incanum C. Müll. Australie

D. Celebesiæ Broth. Celèbes

D. javanicum Broth. Java

D. uncinatum Fleisch. Java

D. Kunerti C. Müll. Brésil

(s) Publié par Husnot dans ses Exsiccata des Antilles ( $\mathrm{n}^{\circ} \mathrm{r} 20$ ) sous le nom de L. Bridelii Hpe. avec l'indication: L. albulum Sull. sec. Bescherelle. Cette plante n'est certainement pas $L$. Bridelii Hpe. publié dans la même collection sous le $\mathrm{n}^{\circ}$ I I 8 et qui appartient au sous-genre Taniodictyon, Sect. Subvittata. Celle-ci est le $L$. serrulatum Brid. (Paris, Index bryol.). Quant au L. subalbulum Ren. ( $\mathrm{n}^{\circ}{ }_{\mathrm{I}} 2 \mathrm{O}$ de Husnot), il est assurement voisin de $L$. albulum Sull.; je crois toutefois devoir l'en séparer à cause de ses feuilles plus étroites, de ses oreillettes plus petites, formées de cellules assez régulièrement hexagonales et de son margo hyalin de moitié plus étroit relativement au L. albulum Sull. de la même île de la Guadeloupe, d'après un échantillon du Muséum de Paris, étiqueté par Schimper lui-même et par Bescherelle. (Voir la planche xxrv). 
Envisagée dans ses grandes lignes, la distribution de nos 3 genres se résume ainsi :

I Les Dicranoloma ont leur centre de végétation dans le bassin austral du Pacifique et quelques espèces dans celui de l'Océan Indien. Ils paraissent manquer au bassin de l'Atlantique.

$2^{\circ}$ Les Leucoloma ont leur centre de végétation dans le bassin de l'Océan Indien, un centre secondaire dans celui de l'Atlantique (mer des Antilles) et quelques espèces dans celui du Pacifique.

$3^{\circ}$ Les Dicnemoloma ont leur centre de végétation dans le bassin du Pacifique (Australie et Malaisie) et une seule espèce dans celui de l'Atlantique (Brésil). Ils manquent jusqu'à présent à l'Océan Indien.

Tels sont les résultats que donne l'examen des matériaux connus actuellement, résultats qui subiront sans doute des modifications à la suite de découvertes ultérieures.

La répartition par grands bassins océaniques plutôt que par grands continents me semble mieux rendre compte de la distribution des Leucoloma et, même en général, des Muscinées.

En effet, en dehors des îles de faible ou moyenne étendue dont le climat est à peu près uniforme dans toute leur superficie, les grandes masses continentales comme l'Afrique, l'Amérique et l'Australie, qui séparent les grands océans, n'ont pas généralement le même régime de pluies sur leurs deux versants Est et Ouest, ni la même Flore à latitude égale. Quand les continents se terminent en pointe étroite comme l'Afrique et surtout l'Amérique, ces caps peuvent être assimilés à des îles où les différences, selon les versants, sont faibles ou nulles; mais, dès que les continents prennent de la largeur, les divergences s'accentuent et tandis que l'un des versants est suffisamment arrosé pour admettre des forêts et une flore muscinale hygrotropicale, l'autre peut ne comprendre, sur de vastes étendues, que des savanes ou même des zones désertiques qui opposent une véritable barrière aux migrations. Il suffit de mettre en opposition, en Amérique, le Brésil avec le Pérou et le nord du Chili, les Pampas de l'Argentine et la Patagonie avec le sud du Chili; en Afrique, le pays des Gallas et des Somalis avec la Guinée, le Natal avec le Kalahari, en Australie le Queensland et la Nouvelle Galle du sud avec l'intérieur et la partie occidentale du continent.

D'autre part les échanges se font en général plus facilement entre terres appartenant au même bassin, si étendu qu'il soit. Les continents qui les séparent sont plutôt des obstacles aux migrations. 
Les centres de végétation caractérisés par l'endémisme de groupes (genres, sous-genres et sections), par l'originalité des types et la richesse en espèces dans une région déterminée fournissent naturellement la note dominante de la distribution géographique. Il serait certainement aussi d'un haut intérêt de reconnaître les affinités des espèces et de suivre aussi les divers courants qu'ont pu prendre les migrations. On constate bien des relations entre diverses terres même très distantes des mêmes grands bassins de l'Atlantique, du Pacifique et de l'Océan Indien, par exemple entre Java et le sud de la Chine, entre l'Australie et le Chili, entre plusieurs archipels de la Polynésie, entre Ceylan et Madagascar; mais il ne semble pas que les documents qu'on possède aujourd'hui soient suffisants pour résoudre la question. Il serait nécessaire d'étudier les espèces d'après des échantillons plus nombreux et plus complets et de mieux connaître l'étendue de leurs variations.

On est obligé de reconnaître qu'en dehors d'un certain nombre de types originaux et très disparates qui servent à caractériser les centres de végétation, beaucoup d'espèces même de provenances très distantes, ne sont séparées que par des caractères légers.

Ces affinités peuvent-elles être expliquées uniquement par les migrations? C'est bien douteux. Assurément les Muscinées possèdent une grande facilité d'expansion par la dissémination des spores; mais encore y a-t-il des limites aux distances au-delà desquelles cette dissémination n'est plus possible. En outre, comme il s'agit ici d'espèces presque toutes dioïques, il faudrait un concours de circonstances vraiment extraordinaire pour que la réunion des sexes puisse se produire très loin du point de départ des spores; d'autre part, des individus unisexués n'ayant d'autre moyen de propagation qu'un processus multiplicatif restreint ne pourraient former que de rares touffes isolées sans possibilité d'extension.

Comment expliquer par l'action seule des migrations l'habitat de quelques espèces sur des points isolés très distants de la région où ces espèces ont leur centre d'origine? Par exemple la présence sur le Kortala, volcan de la Grande Comore d'une Hépatique Symphomitria glossophylla Spruce qui n'était connue que des Andes de l'Amérique du Sud et qui se retrouve identique,jusque dans ses moindres détails anatomiques, sur ce point isolé du Mozambique ? Que ce soit par l'Est ou par l'Ouest que l'on suppose le chemin suivi par les spores, les mêmes impossibilités s'opposent à toute hypothèse de migration.

Sans méconnaître l'importance des moyens de propagation des 
Muscinées par la dissémination des spores dans un rayon étendu, mais cependant limité, il faut reconnaître qu'on ne peut guère expliquer ces affinités de forme extérieure et de structure intime entre des espèces ou races de patries très éloignées qu'en admettant le fait d'une distribution antérieure beaucoup plus large que celle qui existe actuellement.

Pendant longtemps on a cité seulement deux Mousses communes à toutes les terres tropicales: Octoblepharum albidum et Rhinogonium spiniforme. Ce sont en effet les plus généralement répandues et on les trouve partout où sơnt réunies les conditions de climat et d'altitude qui leur conviennent. Mais, à côté de ces 2 espèces, on peut citer un assez grand nombre d'autres dont l'aire de dispersion est très étendue et comprend à la fois tout le bassin du Pacifique et celui de l'Océan Indien. Dans le Prodrome de la Flore de Madagascar j'ai déjà indiqué une vingtaine de ces espèces australes que l'on rencontre çà et là dans toute l'étendue de ces deux grands bassins.

Chaque jour les monographes font ressortir la très grande ressemblance ou même l'identité de types considérés d'abord comme autonomes et habitant des régions séparées par des barrières qui paraissent infranchissables. C'est ainsi que M. H. S. Salmon a encore reconnu récemment que 2 Streptopogon de Madagascar ne peuvent ètre séparés spécifiquement de 2 espèces de patries éloignées, dont l'une de l'Amérique du Sud.

Le fait d'une distribution antérieure plus large s'imposant, le mode selon lequel elle s'est effectuée n'en reste pas moins inconnu et l'on est réduit à des conjectures en ce qui concerne la configuration ancienne des terres dans les vastes océans de l'hémisphère austral. En laissant même de côté l'hypothétique continent de Dumont d'Urville, on peut soupçonner que, lors de la première apparition des Muscinées, des groupes d'îles plus grandes et plus rapprochées, par conséquent plus favorables aux échanges, pouvaient exister. Les arguments tirés de l'étude des Faunes actuelles perdent un peu de leur valeur quand il s'agit des Muscinées dont la première apparition a dû précéder de longtemps celles des animaux supérieurs et, dans l'intervalle, des modifications ont pu se produire dans la configuration des terres.

Les faits de cosmopolitisme sont encore bien plus accusés chez les Lichens et démontrent encore mieux l'existence d'une plus large distribution antérieure. Si l'on compulse le magnifique premier volume que M. l'abbé Hue a consacré à l'étude des Lichens exotiques du MIuséum 
de Paris, on trouve plus de cent espèces, dont une proportion assez notable d'européennes, constatées, soit sous leur forme typique, soit sous des formes affines non spécifiquement distinctes du type, dans des îles très éloignées du Pacifique austral ou de l'Océan Indien et passant même d'un Océan à l'autre, comme de Tahiti à Madagascar.

Des modifications qui s'opèrent dans la Flore muscinale on ne connait sûrement que l'appauvrissement qu'elle subit d'une manière générale et, en particulier dans quelques régions, pour diverses causes: résistance insuffisante de certaines espèces aux influences nuisibles qui peuvent se produire, isolement des sexes dans le cas d'espèces dioïques, enfin et surtout l'action de l'homme qui étend ses cultures, détruit les forêts et par cela même modifie le régime des sources. Dans un intervalle qui ne dépasse pas un demi-siècle, on a pu constater aux Mascareignes la disparition ou la plus grande rareté d'espèces des marécages telles que des Polytrichum et des Sphagnum qui existaient en nombre dans les anciennes collections et manquent plus ou moins aux plus récentes, alors que la grande taille de ces espèces n'auraient pu manquer d'attirer les regards des collecteurs.

La constatation des apports serait d'un haut intérêt; mais elle est bien difficile à réaliser, au moins avec les moyens actuels; j'entends les migrations d'une île à une autre terre distante et qu'il s'agirait de vérifier avec certitude. Des bryologues résidents pourraient seuls entreprendre de semblables observations dans des îles de faible étendue et suffisamment éloignées de toute autre terre, dans les îles madréporiques par exemple dont la Flore est généralement très pauvre en nombre d'espèces et par cela même plus facile à inventorier.

Les travaux monographiques futurs feront sans nul doute ressortir peu à peu l'identité d'un certain nombre d'espèces de régions distantes, distinguées actuellement sous des noms différents. On ne peut prévoir l'importance numérique de ces réductions; mais fût-elle même faible, il ne reste pas moins un fait général qui s'impose tout d'abord à l'esprit: je veux dire l'admirable unité de structure que l'on trouve dans la plupart des genres riches en espèces. Quand on voit, entre des espèces même appartenant à des Domaines bryologiques éloignés et très différents, des affinités si étroites qu'il faut, pour les distinguer, un examen anatomique minutieux, on est porté à croire à une large distribution primitive des types qui se seraient ensuite plus ou moins différenciés avec le temps surtout dans le cas où un changement dans la configuration des terres les aurait isolés, en transformant en îles des surfaces continentales plus ou moins étendues. 
La raison primordiale de la distribution actuelle des Muscinces devant se chercher dans des causes anciennes qui n'ont pas eu de témoins - large diffusion et existence de centres de végétation - la part qui revient aux migrations n'en existe pas moins. Sans parler même de la propagation à distance par la dissémination des spores ou, dans quelques espèces, de propagules cloisonnés sur des contiments très étendus sans interruptions, comme l'Europe et l'Asie boréales, quelques échanges se conçoivent fort bien entre des îles médiocrement distantes, encore que ces échanges soient très difficiles à constater sûrement; mais on doit reconnaître que les migrations ne jouent qu'un rôle secondaire et ne s'effectuent que dans des cas déterminés et à des distances limitées, au-delà desquelles la dissémination des spores par les courants aériens devient aléatoire et incertaine, tandis que la propagation par les oiseaux migrateurs reste à l'état de fait exceptionnel.

Cette action des oiseaux migrateurs s'étend sur d'immenses distances: car, habitant pour la plupart, des zones tempérées, ils sont obligés de passer chaque année d'un hémisphère à l'autre, selon l'alternance des saisons, afin de trouver sans interruption les conditions essentielles de leur existence ; mais ces migrations ne sauraient se comprendre que dans la direction des méridiens. Quelle part faut-il attribuer aux grands palmipèdes des mers australes qui peuvent parcourir de grandes distances dans des sens divers, à la poursuite de bancs de poissons? Reste la circulation des navires dont on pourra rechercher les effets en suivant attentivement les modifications qui pourront se produire dans la Flore muscinale des environs des ports les plus fréquentés. A cet ordre d'idées se rattache le fait suivant: dans l'Est de la France (Haute Saône), vers ı 860 , on a, pour la construction d'une voie ferrée, creusé une tranchée dans les grès du Lias. Quelques années plus tard, j'ai vu les parois rocheuses de cette tranchée recouvertes d'une végétation bryologique fournie et, parmi les espèces que j'y ai reconnues, plusieurs ne croissaient pas dans le voisinage, même dans un rayon assez étendu et l'une d'elles Dicranella Grevillei, d'origine subalpine, est inconnue ailleurs dans le département. A quelques lieues plus loin, à Vesoul, sur les bords d'un canal de dérivation longeant la même voie ferrée, on a planté des arbres dont les troncs ont été envahis par de nombreuses touffes fertiles de Pylaisia polyantha, espèce assez répandue dans la plaine d'Alsace, mais qui, auparavant, n'existait pas à Vesoul et qui paraissait même fort rare dans le département. Il n'est pas douteux que ces introductions ne soient dues à la circulation des trains; on conçoit donc que celle des navires puisse produire les mêmes résultats. 
Il me reste à citer un fait très significatif, je veux dire l'introduction dans deux îles italiennes voisines de la Sicile de deux espèces tropicales récemment reconnues par mon ami Bottini et qui toutes deux ont été trouvées dans des cavernes humectées par des vapeurs chaudes dues à des influences volcaniques. C'est, dans l'île de Pantelleria un Calymperes, et, dans celle de Stromboli, un Meteorium (Barbella), genres dont la présence n'était pas même soupçonnée dans le bassin Méditerranéen. L'introduction de ces espèces qui pourront se maintenir peut être, grâce aux conditions toutes spéciales de la station, est ici évidente (1). Est-elle due à la circulation des navires très active dans ces parages ou aux oiseaux migrateurs? Si ces espèces sont retrouvées plus tard dans le centre de l'Afrique, la question sera tranchée dans ce dernier sens. Dans tous les cas, il était très important de pouvoir constater, d'üne façon sûre, un fait de migration par un moyen autre que le transport des spores par les courants aériens; mais ces constatations précises, encore rares, deviendraient-elles plus nombreuses, que les migrations resteraient à l'état de faits sinon exceptionnels, du moins accidentels. Si la propagation des spores à longues distances se produisait fréquemment et facilement, on n'observerait pas un cachet d'endémisme si accentué dans la Flore muscinale des îles de l'hémisphère austral et l'explication des stations disjointes très éloignées, surtout dans la direction des parallểes, me semble toujours devoir être cherchée dans l'hypothèse d'une distribution antéricure plus large.

(1) La présence d'un Hypopterygium dans l'île de Vancouver (Colombie anglaise) constatée par le Dr N.C. Kindberg est un fait de même ordre dû aux oiseaux migrateurs, 




\section{PRODROME DE $X A$}

FLORE BRYOLOGIQUE DE

MADAGASCAR

DES MASCAREIGNES

ET

DES COMORES

\section{SUPPLÉMENT \\ PAR}

Ferdinand RENAUD 

Les documents que j'ai pu recueillir sur la Flore muscinale des îles austro-africaines depuis la publication du Prodrome (I 897), sans être considérables, ont fourni cependant un appoint de soixante-quinze espèces, la plupart nouvelles, et de 4 genres non représentés encore dans ce Domaine. La guerre de I 895-I 896 ayant dispersé mes correspondants de Madagascar, il m'a été difficile d'y trouver de nouveaux collecteurs et c'est au zèle et à l'activité de M. le général Paris, heureusement secondé par $M$. le général Gallieni, alors gouverneur de lîle, que la plus grande partie des nouveaux matériaux a pu être obtenue.

En 1 900, M. Macé, officier de Marine, profitant d'une escale à la Grande Comore, a fait, à la prière du regretté M.H. de Poli, l'ascension du volcan le Kortala et en a rapporté une trentaine d'espèces. Cette même année, $M$. Corbière m'a communiqué quelques espèces récoltées à Diego Suarez par le capitaine Leloutre. Mon ami Cardot a reçu de M. Mathieu une petite collection provenant de Maroantsetra,au fond de 
la baie d'Antongil. Parl'intermédiaire de M.le Dr Levier, nous avons eu communication des récoltes faites dans les forêts du versant oriental, notamment la forêt d'Analamazaotra, par MM. Perrot et Forsyth Major et, par celui de M. l'abbé Lacouture, des espèces assez nombreuses et fort intéressantes collectées par le Rev. P. Villaume dans le pays des Betsileos. Il me reste à citer quelques spécimens reçus de M. Dalincourt, provenant du plateau central de Madagascar et du $D^{r}$ Farlow de l'Université de Cambridge (Massa chusets) 2 échantillons de l'île Maurice, parmi lesquels le Schlotheimia quadrifida Brid. dont l'habitat dans les Mascareignes avait été contesté par Bescherelle.

M. Thériot,qui a eu roccasion d'étudier plusieurs espèces de Madagascar, a bien voulu nous communiquer aimablement ses intéressantes observations, qui seront consignées à leur place dans le cours du volume.

Les Sphagnum ont été déterminés par l'éminent spécialiste, M. Warnstorf.

Durant ces dernières années ont été publiés deux ouvrages de systématique très importants et d'après lesquels je dois modifier, pour quelques familles, la classification adoptée dans le Prodrome. Ce sont d'abord les savantes Recherches anatomiques sur les Leucobryacées de mon collaborateur et excellent ami M. Cardot, ainsi que Les Leucobryacées de Madagascar du même auteur (Bulletin de l'herbier Boissier, I g04).J'adopte sa classification, pensant que s'il est préférable de chercher les caractères génériques parmi ceux qui présentent le plus de fixité, le plus de généralité et qui souffrent le moins d'exceptions, on ne saurait imposer de 
principe absolu sur le choix des organes dont il faut examiner les modifications. En génétal, c’est la structure du péristome qui offre les caractères les plus importants, les plus fixes; mais ailleurs, chez les Leucobryacées par exemple, l'histologie des organes de végétation offre une base plus stable pour établir les coupes génériques, tandis que les variations du péristome ont beaucoup d'amplitude.

Le deuxième ouvrage auquel je fais allusion est le "Genera» de M. Brotherus (Pflanzen Familien), impatiemment attendu depuis longtemps et dont la publication n'est pas encore achevée. Pour mettre mon Prodrome en concordance complète avec ce magistral ouvrage et avec les remarquables travaux de systématique de M. Fleischer, il me faudrait le remanier complètement, ce qui mènerait trop loin dans un supplément. Il n'y a d'ailleurs de modifications très importantes que dans la Famille des Neckeracées, qui sera exposée à nouveau dans le cours de ce volume en indiquant la concordance des nouveaux et anciens genres. Les coupes génériques nouvelles, même si quelques-unes doivent être plus tard considérées comme sous-genres, facilitent beaucoup la compréhension de cette Famille, formée auparavant de genres fondés les uns après les autres à des époques différentes, sans principe général, par des auteurs différents dont les plus anciens ne connaissaient que peu d'espèces, de sorte qu'avec les éléments déjà très disparates dont elle se compose on n'avait pu aboutir qu'à un amalgame de genres sans grande liaison. Il restera longtemps encore la difficulté de classer beaucoup d'espèces stériles par la seule considération des analogies de leurs organes de végétation, 
c'est-à-dire avec plus ou moins de doute, jusqu'à ce qu'on ait découvert les fructifications. Cette classification ne constitue pas moins un notable progrès.

Nos exsiccata (Musci mascareno-madagascarienses), qui ne comprenaient que i 50 numéros au moment de la publication du Prodrome ont été augmentés d'une centurie et distribués jusqu'au $\mathrm{n}^{\circ} 250$.

Enfin M. Cardot et moi avons, de I 898 à i 905 , publié un Atlas des Mousses de Madagascar faisant partie de l'Histoire physique et naturelle de Madagascar par M. Alfred Grandidier. Cet Atlas comprend jusqu'à présent r 63 planches, en 5 volumes; je fais des vœux pour que mon ami Cardot puisse le compléter par un $6^{\mathbf{e}}$ volume. Aucun texte n'ayant paru encore, je crois utile de faire figurer dans ce supplément l'explication des i 63 planches éditées, afin de faciliter aux lecteurs l'usage de l'Atlas. 
PRODROME

DE LA

\section{FLORE BRYOLOGIQUE}

\section{DE \\ MA D A G A S A R}

DES MASCAREIGNES ET DES COMORES

\section{SUPPLÉMENT}

\section{RÉGIONS EXPLORÉE}

\section{Madagascar}

Les matériaux concernant cette île, composés d'une foule de petites fractions, proviennent de localités si diverses qu'il serait trop long de les énumérer. Pour la plupart, d'ailleurs, M. le général Paris a cité dans la Revue bryologique les noms des collecteurs qui seront reproduits plus loin en regard de ceux des espèces. Je dois toutefois faire une exception pour les renseignements concernant des régions non ou peu explorées auparavant et dont l'ensemble est suffisant pour mieux faire ressortir le faciès bryologique. C'est en particulier le cas de la province de Betafo dans la partie Ouest du plateau central qui incline déjà ses pentes vers le Mozambique, et où M. l'administrateur Garnier Mouton a collecté Pottia(?) punctulata Ren. et Par., Schlotheimia acutifolia Ren. Par., quelques brins du curieux Calyptothecium planifions Ren. Par. reconnu peu de temps après au Congo belge, puis dans le bassin 
supérieur de la Mania Leucobryum Galinoni Card. et Par., Hyophila angustifolia Par. Ren., Fabronia Garnieri Ren. Par., Thuidium trachynoton Ren. Par., Isopterygium Manice Ren. Par., cette dernière espèce très voisine de $I$. taxithelioïdes C. Müll. de l'Himalaya, Taxithelium hirtellum Par. Ren.; enfin, sur ce même versant littoral ou sublittoral du Mozambique, dans les cercles de Majunga ( $15^{\circ} 40^{\prime}$ ) et de Maintirano (1 $\left.8^{\circ} 30^{\prime}\right)$ : Fissidens Zippelianus D. M. et sa subspecies Savellii Par. Ren., Ochrobryum Sakalavum Card. Par., Leucobryum cucullatum Broth., Octoblepharum albidum Hedw., Pottia tuberculosa Ren. Par., Hyophila clavicostata Ren. Card., Hyophila Dorrii Ren. Card., Hyophila sakalavensis Par. Ren., Hydrogonium Brotheri Ren. Par., Epipterygium diversifolium Ren. Par., ces 2 derniers genres nouveaux pour notre Domaine, Thuidium subscissum C. M., Ectropothecium spharocarpum C. Müll. Splachnobryum inundatum Besch., Fissidens Motelari Ren. Card., Fissidens obsoletidens Besch., Calymperes erosulum Ren. Par.

Ce groupe d'espèces, auxquelles il faut joindre celles déjà énumérées dans le Prodrome pour la zone des Savanes occidentales, forme un ensemble d'un peu plus de 30 espèces qui représente ce qu'on connaît aujourd'hui de la Flore du versant du Mozambique. On ne possède malheureusement aucun renseignement sur la ligne de collines calcaires peu élevées qui court du Nord au Sud à mi-distance du bord du bombement central et du littoral du Mozambique (Monts Bemaraha). Elle fournira probablement quelques espèces spéciales en raison de sa constitution géologique, l'élément: calcaire: dont elle est formée étant l'exception à Madagascar.

La région Sud-Occidentale de la grande île reste toujours inexplorée. Sur le versant méridional, au pays des Bara situé au Sud de Fianarantsoa, quelques espèces collectées aux environ d'Ivondro vers $23^{\circ}$ de latitude portent le:cachet de la région des forêts :

Trichostomum Pennequini Ren. Par. Leucoloma punilum C. M.

Leucobryum molle C. M.

Fissidens ovatus Brid.

Syrrhopodon sparsus Ren, Card.

Bryum homalobolax C. M:

Porotrichum Chenagonii C. M.

Thuidium Chenagoni Ren. Card.

Fabronia fastigiata Ren. Card.

Stereophyllum limnobioides R. Card.

Enfin, de l'extrême Sud de l'île dont il aurait été très important d'obtenir quelques récoltes, 5 : espèces seulement ont lété rapportées: 
c'est d'abord un beau et robuste Camprlopus nouveau: C. Gallienii Par., puis Leucoloma fuscifolium Besch., Leucophanes Renauldii Card., Leucobryum molle C. M., Ectropothecium Chenagoni Ren. Card. dejà signalés dans toute l'étendue de la zone des forêts orientales jusqu'à Diego Suarez. Peut-on conclure de cet embryon de données que la Flore de cette zone se modifie peu dans toute sa longueur méridienne? Ce qui est acquis par les constatations faites à des latitudes distantes, c'est que la Flore comprend de nombreux éléments communs qui ont pu se propager de proche en proche le long de la Chaîne sans que des différences climatériques importantes y aient mis obstacle. Assurément, il n'y a pas partout identité et c'est ainsi que la pointe Nord de l'île, surtout depuis la baie de Passandava, possède un certain nombre d'espèces communes avec les Comores, notamment des Jagerina, Hildebrandtiella, pour ne citer que les plus caractéristiques. D'autre part, le climat plus tempéré de Fort Dauphin (lat. $25^{\circ}$ ), dont la température moyenne annuelle est de $3^{\circ}, 5$ inférieure à celle de Diego Suarez, peut admettre des espèces spéciales et une représentation plus riche de genres européens. On sait que dans quelques vallées des environs de Fort Dauphin (vallée d'Ambolo) la végétation est verdoyante et bien fournie et que la quantité d'eau tombée annuellement est égale ou supérieure à I mètre ; mais, pour le reste, on est réduit à des hypothèses et au regret de ne pas posséder plus de renseignements sur cette région certainement intéressante.

Dans le pays des Betsileos qui fait suite au Sud au plateau de l'Imerina, le P. Villaume a collecté une cinquantaine d'espèces dont plusieurs nouvelles :

Anoectangium madagassum R. C. Leptotrichum madagassum R. C. Dicranoloma scopareolum (C. M.) R. Leucoloma ambreanum R. C.

L - subtuberculosum Ren.

L - bifidum Brid.

Fissidens ovatus Brid.

F-Boivini Besch.

Leacobryum molie C. M.

L-Boryanum Besch.

$\mathrm{L}$ - madagassum Besch.

Ocioblepharum albidum $\mathrm{Hdw}$.

Campylopus polytrichoïdes de Nor.
Holomitrium borbonicum. Hpe

Macromitrium tenue Brid.

Schlotheimia trichophora R. C.

Philonotis laxissima C. M.

Brachymenium Heribaudi R. C.

B - philonotula Hpe

Bryum spinidens $\mathrm{R}$. C.

Pilotrichella biformis Hpe

P. - imbricatula C. M.

$\mathrm{P}$ - longinervis $\mathrm{R}$. C.

Porotrichum mucronulatulum C. M.

Papiilaria fuivastra Besch.

Hookeria Auberti P. B. 
Fabronia Villaumii R. C.

Erythrodontium Lacouturei R. C.

Thuidium trachynoton R. P.

Pseudoleskea obtusiuscula R. C.

P - subfilamentosa Kiaer

Leptohymenium Ferriezii Besch.

L - Borgeni Kiaer

Brachythecium Borgeni Hpe

B - atrotheca Duby

Rhynchostegium distans Besch.

Ectropoth. nano crista castrensis C.M.

Rhaphidostegium Duisaboanum Bes.

Microthamnium serratum P. B.

M. brachycarpum R. C.

Bryum erythrocaulon Schwgr.
Bryum suberythrocaulon R. C. Mnium australe Besch.

Bryum homalobolax C. M.

Orthodon borbonicus Bory.

Barbula subrufa R. C.

Papillaria pseudo fulvastra C. M.

Trachypus serrulatus (Brid.)

Acanthocladium flexile R. C.

Rhacopilum madagassum $R$.

Ectropothecium Chenagoni R. C.

Pógonatum obtusatulum C. M.

Phyllogonium viscosum P. B.

Leucoloma ochrobasilare Ren.

Brachymenium lineale Ren. Card.

Entosthodon Renauldii Thér.

Si à cette liste on ajoute celles déjả citées dans le Prodrome,on arrive, pour ce vaste pays des Betsileos, à une centaine d'espèces formant un ensemble composé à la fois d'éléments de la zone supérieure des forêts et du plateau central. Cette Flore paraît aujourd'hui assez bien connue dans ses grandes lignes; mais elle n'a certainement pas encore livré toutes ses richesses.

L'exploration des forêts du versant oriental de Madagascar n'a fourni que peu d'espèces nouvelles: Leucoloma Garnieri Par. Ren., Campylopus Verdolini Par. Ren., Hyophila leioneura Ren. Par., Bryum subgracilescens Ren. Par., Bryum angustissimifolium C. Müll., Leucoloma leptocladum Ren., Leucoloma Levieri Ren., Trichosteleum laviusculum Ren.Card.,Calymperes Mathici Ren.Card.,Campylopus pseudo virescens Ren. Card., Pilotrichella pseudo imbricata C. Müll., Sphagnum grandirete W., puis de l'île Sainte-Marie, Leucoloma Brotheri Ren., Hyophila Girodi Ren. Card.

En résumé, la Flore bryologique de Madagascar et îlots annexes qui comprenait 425 espèces, se trouve augmentée, par suite de toutes ces additions, de plus de 75 espèces, en majorité nouvelles, ce qui en porte le nombre total à 500 au moins.

Si quelques-unes de ces espèces doivent être plus tard supprimées comme ne possédant pas une valeur spécifique suffisante, il est probable que cette diminution sera largement compensée par les apports provenant des régions de la grande île non explorées encore et en particulier de la partie méridionale située en dehors du cercle des tropiques. 
Le Fissidens Zippelianus D. M. de Java, constaté sur le littoral du Mozambique est une espèce de plus à ajouter à celles communes aux deux Domaines; encore se présente-t-il le plus souvent sous une forme (subsp. Savellii Par. Ren.) qui n'est pas identique à celle de Java ou cette espèce est d'ailleurs assez polymorphe. Mais si, même par la com paraison plus étudiée des formes affines des deux Domaines, on arrivait à augmenter le nombre des espèces communes, il ne faudrait pas exagérer l'importance de ces relations basées sur la présence d'espèces introduites accidentellement ou dont l'aire de dispersion est étendue, tandis que le faciès bryologique javanais est manifesté surtout par des genres très spéciaux dont quelques-uns australiens et polynésiens manquant totalement à Madagascar, qui possède en revanche, outre ses genres et nombreuses espèces endémiques, un cachet rappelant beaucoup plus celui du continent africain.

\section{Grande Comore.}

M. Macé,officier de marine,qui a exploré en igoole volcan le Kortala, a fait la majeure partie de ses récoltes dans la région forestière à une altitude moyenne de 600 mètres; il s'est élevé toutefois au-dessus de la zone des forêts jusque vers 2300 mètres où il a collecté 4 espèces marquées d'un astérisque dans la liste suivante:

Dicranella madagassa Ren.

Leucoloma Isleanum Besch.

Leucoloma Comoræ Ren.

* Campylopus polytrichoïdes de Not.

- var altecristatus Ren. Card.

* Campylopus Orzeszkoanus R. Card. Leptodontium epunctatum C. Müll. Leucobryum mayottense Card.

Braunia entodonticarpa C. Müll.

Macromitrium subpungens C. Müll.

* Bryum vinosulum Besch.

Anomobryum julaceum

* Philonotis comorensis C. Müll.

Polytrichum comorense C. M.

Pogonatum Macei Ther.

Jaegerina stolonifera C. Müll.
Prionodon ciliatus Besch.

Hildebrantiella endotrichelloïdes

C. Müll.

Aerobryum lanosulum C. Müll.

Papillaria Boivini Besch.

Papillaria floribundula C. Müll.

Pilotrichella biformis $\mathrm{Hpe}$.

P - pseudo imbricata C. Müll.

P - ampullacea C. Müll.

Porotrichum comorense C. Müll.

Neckera Comoræ C. Müll.

Thuidium subscissum C. Müll.

Sematophyllum megasporum Duby

Microthamnium madagassum Besch. Rhacopilum plicatum Ren. Card. Hypoterygium Sp. 
Cette lisste fournit un appoint de 20 espèçes à ajouter aux 37 ,espèces indiquées dans le Prodrome. La Flore de la Grande Comore, dont on ne connaissait que r 4 espèces avant la publication de ce dernier, comprend donc aujourd'hui 57 espèces, chiffre équivalent a ce qui a été constaté à Anjouan, mais qui sera certainement augmenté plus tard. Je signalerai en particulier Braunia entodonticarpa C. Müll, trouvé d'abord au Kilimandjaro et qui appartient à un genre non encore représenté dans notre Domaine, puis une Hépatique: Symphiomitra glossophylla Spr., espèce des Andes de l'Amérique australe qui, par une exception singulière, se retrouve au sommet du volcan Kortala, vers 2300 mètres d'altitude.

Le nombre dés Hépatiques de notre Dómaine s'est enrichi de 29 espèces, la plupart nouvelles, déterminées par M. Stephani.

\section{WNUMERATION DES ESPECES}

\section{ACROCARPI}

\section{Gen. Anœctangium.}

A Mariei Besch., Nossi-Bé, Helleville, R. Cailté, rgoo.

A. madagassum Ren. Par.-E. minimis. Caulis 2-5 mill, longus. Folia remotiuscula, comalia densius conferta, ligulato-linealia parva, I/2 mill. longa, o, 5 mill. lata apice obtusa vel rotundata, rarius obtuse acuminata. Costa sub apice evanida. Rete usque ad basin fere obscuro, indistincto; papillis densissimis ad margines et dorso costæ prominentibus. Pedicellus 2 mill. longus pallidus. Capsula minuta ovata $3 / 4$ mill. longa.

Madagascar: Ambositra, Rev.Villaume (Hb. Lacouture); Ankazobé, Fihaonana (Hb. Paris, I901).

C'est avec $A$. impressum $\mathrm{Hpe}$ que cette espèce a le plus d'analogie. Elle s'en distingue par ses feuilles très obtuses et le tissu indistinct presque jusqu'à la base obscurci par des papilles très denses. Dans $A$. impressum Hpe la taille est un peu plus robuste, les feuilles sont aiguës, rarement quelques-unes subobtuses. Le tissu est beaucoup moins obscur et on distingue le plus souvent chaque cellule, munie d'une large papille festonnée. Béc de l'opercule fortement arqué. 
L'Anoectangium borbonense Besch. de La Réunion, dont la taille est parfois très petite, a les feuilles plus longues ( $r$ mill.) aiguës. Le tissu est obscur mais non jusqu'à la base, celle-ci composé de cellules brièvement rectangulaires hyalines. Le pédicelle est beaucoup plus long (jusqu'à 8 mill.), la capsule est aussi plus allongée.

L'A. Mariei Besch. de Nossi Bé a aussi les feuilles plus longues, aiguës, hyalines dans tout le $\mathrm{I} / 3$ inférieur qui est formé de cellules rectangulaires allongées, lisses. La nervure est couverte sur le dos de grosses papilles très saillantes.

L'A. rufoviride Besch. des Comores a également les feuilles plus longues (I mill.) nettement rétrécies acuminées, aiguês. Le tissu est obscur, peu distinct si ce n'est à l'extrême base qui est composée de cellules carrées, hyalines, mais portant au centre une petite papille. Le pédicelle est aussi beaucoup plus long ( 12 mill.), la capsule plus étroite. Les papilles sont peu saillantes sur les marges de la feuilles et le dos de la nervure.

D'autre part, il n'y a aucun risque de confusion avec les A. rhaphidostegium C.M. des Comores, A. Humbloti R. C. de la Grande Comore et A. mafatense R. C. de La Réunion, qui sont des espèces plus robustes à feuilles plus longues $(2-2$ I/2 mill.) nettement acuminées, aiguês, à long pédicelle (Io-12 mill.), à capsule étroite plus allongée.

\section{Gen. Dicranoloma.}

D. patentifolium Ren. et Par. (Planche v). - Cæspites laxiusculi luteo-virides. Caulis elongatus 6-1o cent. longus, erectus, flexuosus, simplex vel parce divisus. Folia remotiuscula caulem haud abscondentia falcato-patentia vel undique patula, comalia falcata 5-6 mill. longa, e basi oblonga longe lanceolato-subulata, marginibus e medio late inflexis, superne convolutaceis, serratis ; costa angusta basi $40 \mu$ lata superne dorso argute serrata, rete pallido, perdenso, subscarioso, scoparioideo, e cellulis elongatis porosis efformato, alaribus rufo purpureis quadratis vel breviter rectangulis subcatenulatis auriculas excavatas sistentibus; limbo hyalino angustissimo interdum obsoleto $6 \mu$ lato e cellulis 2-seriatis composito. Cætera desunt.

\section{Madagascar : région de Mandritsara, I9o I (Hb. Paris).}

Diffère du $D$. scopareolum (C. M.) Ren. de la même région par les feuilles fortement dentées en scie, ainsi que le dos de la nervure dans le tiers supérieur. Se distingue du D. drepanocladium (C. M.) Broth. du Kilima N' Djaro par un port différent, plus robuste, par les feuilles espacées, étalées-falciformes, plus étroites, munies d'oreillettes plus grandes, excavées, par le dos de la nervure pourvu de dents plus fortes disposées sur $2-3$ rangs.

D. scopareolum (C. M.) Ren.

Madagascar: Ambohimiera, leg. Froissart, 1905 (Hb. Paris); Ambositra (Betsileo), Rev. Villaume, igo6. (Hb. Lacouture). 


\section{Gen. Leucoloma.}

L. BIFIDUM Brid.

Madagascar: Ambositra, Rev. Villaume, 1906 (Hb. Lacouture).

L. (Teniodictyon) leptocladum Ren.(Pl. II). - Caulis gracilis, 2-3 centim. longus, flexuosus, apice attenuatus, ramis nonnullis, gracillimis proditus. Folia caulina erecta, apice vix subsecunda, haud crispata, 2,5 mill., longa, late ovata, sat subito in subulam remote denticulatam attenuata, ramea breviora et angustiora; costa breviter excurrente, cellulis internis distinctis oblongo-quadratis (diam. $2 \mathrm{I} / 2 \mu$ ), alis membranaceis e cellulis plerumque parenchymatosis 15-18-seriatis reticulatis, papillis dorso granulosis, limbo hyalino subnullo. Cætera ignota.

Madagascar: province de Tamatave, forêt de Fito, Perrot (Hb. Levier).

Espèce de la section Euvittata, se distinguant à première vue par ses tiges grêles, munies de rameaux effilés, et par ses feuilles dressées-appliquées, légèrement secondes par la pointe seulement. Diffère du L. subbifidum Ren. par les feuilles plus courtes et plus larges, par la subule denticulée dans toute sa longueur et par la nervure plus brièvement excurrente.

I. (Taniodictyon) ochrobasilare Ren. (Pl. II). - Cespites laxi, inferne rufo-castanei, superne lutescenti-virides, caulis $4-5$ cent, longus, plus minus divisus. Folia sicca apice incurvato-crispula, 2, 75-3,5 mill. longa, ovato-oblonga, cuspidata, superne denticulata, costa cum apice finiente vel paululum excedente, lamina interna sat longe supra basin pallida, vix papillosa (unde nomen), cellulis internis mediis et superioribus utraque pagina papillosis, alis membranaceis e cellulis $15-20$ seriatis compositis, limbo hyalino angustissimo $(6-10 \mu)$, e cellulis $\mathrm{I}-3$ seriatis formato, interdum subnullo, papillis ventralibus rotundatis, dorsalibus bifidis magis prominentibus. Cætera ignota.

Madagascar : Ambondromba, Betsileo, Rev. Talazac, 1894 ; Ambohimitombo, Tanala, alt. i 350-1450 m., Forsyth Major (Hb. Levier).

Espèce de la section Euvittata, bien caractérisée par les feuilles papilleuses sur les deux faces, et les papilles dorsales bifides, contrairement à l'habitude du. sousgenre, ainsi que par la lame interne qui cesse d'être papilleuse à partir du tiers inférieur, et qui est formée vers la base de cellules oblongues. Ressemble par le port au 
L. subchrysobasilare C. Müll., de la même région, mais outre les caractères précités, les feuilles sont plus brièvement cuspidées, et la nervure non ou à peine excurrente.

L. (Taniodictyon) thraustum Hpe (Pl. Iv). - Cæspites laxi. Caulis flexuosus r-5 cent. longus, dichotome divisus. Folia flexuosa, incurvato-subcrispula, 2-2 I/2 mill. longa, oblongo lanceolata longiuscule subulata, integra vel subintegra. Costa breviter excurrente basi circa 45 lata. Lamina interna latere bene separata basin versus pallida, vix papillosa. Limbo hyalino 14 - $16 \mu$ lato e cellulis $2-3$ seriatis efformato. Cellulis alaribus longiuscule rectangulis, internis distincte papillosis, papillis densis subbifidis dorso parum prominentibus. (Diagnose faite d'après l'échantillon original de Hampe dans l'herbier C. Müller, conservé au Museum de Berlin).

Cette espèce appartient bien, comme je le soupçonnais (Cfr. Prodrome, page 87) à la section Vittata. Elle est très voisine de L. bifidum Brid. dont elle se distingue par un port plus robuste, par les tiges divisées par dichotomies, par les feuilles plus larges plus brièvement subulées et crispulées à l'état sec.

Voisine aussi du L. silyaticum Ren. dont elle diffère par les feuilles moins larges à subule plus longue entière ou subentière, à margo un peu plus étroit. Cellules internes plus petites. Elle se distingue d'autre part du $L$. ocltrobasilare Ren. par la cuspide entière, par la lame interne obscure descendant beaucoup plus bas, enfin par les papilles à peine saillantes.

L. (Taniodictyon) holomitrioides C. Müll. in hb. - Caulis $\mathrm{I}=3$ cent. longus, pluries divisus, ramulos graciles foliis angustis, setaceis apice flexuosis præditos emittens. Folia erecta, rigida, haud crispula e basi ovata vel oblonga longe subulata, pro more superne conduplicatotubulosa, subula sæpius flexuosa dentibus remotis acutis prædita. Costa basi $29-35 \mu$ lata pro more longe excurrente. Lamina interna sæpius in medio inferiore pallida et vix papillosa, in medio superiore plus minus obscura e cellulis dorso convexis minute papillosis efformata. Limbo hyalino angusto $7-9 \mu$ lato e cellulis 2-3 seriatis composito. (Diagnose faite d'après un fragment de l'échantillon original de C. Müller, du Museum de Berlin).

La place que j'avais assignée provisoirement à cette espèce dans le Prodrome (page 75 ), alors que je n'avais pas examiné d'échantillon authentique, est inexacte. Elle appartient à la section Viftata et a été collectée à Alamazantroskoven par Borchgrewinck. Elle est caractérisée par ses feuilles de longueur très inégale, dressées, nullement crispulées terminées par une très longue subule tubuleuse. La lame interne est très pâle et presque dépourvue de papilles dans la moitié inférieure de beaucoup de feuilles, mais, dans d'autres feuilles, papilleuse presque jusqu'à la base. 
Cette espèce diffère du L. subchrysobasilare C. Müll. par la taille moins élevée, par les feuilles raides, par la subule plus longue, plus fortement dentée et par la base de la lame interne pâle.

L. (Taniodictyon) subtuberculosum Ren. - Cespites laxi pallide virides. Caulis flexuosus, erectus dichotome ramosus 3-4 cent. altus. Folia laxiuscule conferta, sicca crispula, 2 1/2-3 mill. longa $3 / 4$ mill. lata ovato-oblonga, cuspidata, costa basi $45-47 \mu$ lata breviter excedente, superne dentata. Lamina interna obscura dense utraque pagina papil losa, paulo supra basin desinente, transverse haud rugulosa. Limbo hyalino angusto i 3-i $6 \mu$ lato, e cellulis 3-4 seriatis composito. Cellulis alaribus scalariformibus, haud incrassatis. Capsula ovato-globosa in pedicello brevissimo, æquilongo vel breviore, erecta rufo-castanea.

Madagascar : Ambrosita (Betsileo), Rev. Villaume, I 906 (Hb. LACOUTURE).

Cette espèce qui appartient à la section Obscura diffère du L. Thuretii Besch par les touffes lâches, par les tiges allongées, dichotomes, par l'acumen plus fin, la nervure plus étroite, par les cellules des oreillettes à parois non épaissies, par le margo un peu plus étroit. Les cellules internes forment 2 bandelettes arrivant sans se rétrécir jusqu'un peu au-dessus de la base. Pour ce dernier motif il est préférable de classer cette espèce dans la section Obscura plutôt que dans le sous-genre Sphenodictyon dont elle se rapproche par d'autres côtés.

Diffère du $L$. tuberculosum Ren. par les feuilles non ridées en travers et par les papilles denses simplement bifides et beaucoup moins saillantes sur la face dorsale.

L. Sancte Marie Besch. var. 1eptolimbatum Ren. - A planta typica differt subula robustiore, evidentius denticulata, limboque hyalino multo angustiore.

Madagascar : Forêt de Ambohimitombo: $\mathrm{D}^{\mathrm{r}}$ Forsyth Major. (Hb Levier).

L. (Sphenodictyon) acutum Mitt. - D'après l'examen d'un specimen authentique, $j$ 'ai pu constater que cette espèce qui est restée à l'état de nomen est identique à $L$. Crepini Ren. Card. Aux localités de Madagascar citées dans le Prodrome pour ce dernier, il faut ajouter celle d'Antseranandratzy, Igo I (Hb. Paris).

L. (Sphenodictyon) Levieri Ren. (Pl. III). - Cespites laxissimi, intus rufescentes, superne pallide lutescenti-virides. Caulis 3,54,5 cent. altus plus minus divisus. Folia inferiora patula, superiora 
undique subflexuosa, 3-4 mil. longa, oblongo-lanceolata, nervo excurrente longe cuspidata, superne subtiliter denticulata, marginibus superne involutis, lamina interna transverse plicata vel rugulosa,inferne truncata, vix attenuata, vage supra basin desinente, cellulis internis rotundatis (diam. $3 \mu$ ), basilaribus elongatis, membranam scariosam sistentibus, limbo hyalino angustissimo (10-1 $2 \mu)$, e cellulis 2-3-seriatis composito, papillis bifidis dorso corrugato maxime prominentibus, pro more in lineas rugulosas transverse dispositis. Cætera ignota.

Madagascar : province de Tamatave, forêt de Fito, Perrot (Hb. Levier).

Très voisin du $L$. tuberculosum Ren., dont il se distingue par le port, par les feuilles plus longues, oblongues-lancéolées, longuement cupidées par l'excurrence de la nervure, à subule subaiguë presque entière ou finement denticulée.

L. (Sphenodictyon) tuberculosum Ren. (Pl. II).

Madagascar : Fianarantsoa (Hb.Paris) ; Ambositra, Rev. Villaume, 1905.

L. (Syncratodictyon) CUNEIFolium Hpe.

Madagascar: Province d'Ambositra, Onzorozoro, Frissard (Hb. Paris) Betsileo, Rev. Villaume, 1906.

Pédicelle pourpre long de 8 mill. Capsule cylindrique châtain foncé ou brune, dressée, longue de 2 mill. atténuée à la base; opercule conique atténué en un long bec droit.

L. (Syncratodictyon) Ambreanum Ren. Caru.

Madagascar : Province d'Ambositra, Onzorozoro, Frissard (Hb. Paris) Betsileo, Rev. Villaume, I 906.

L. (Syncratodictyon) DELICATULUM Ren.

Madagascar : Côte N.-O. Mossé, I 899 (Hb. de Poli).

L. (Syncratodictyon) PERsecundum C. Müll.

Madagascar: Moramanga, Igor (Hb. Paris).

L. (Syncratodictyon) ALbocinctum Ren. Card.

Madagascar: Tsongatsa près Andevorante ; Moramanga, I901, (Hb. Paris). 
L. (Syncratodictron) Brotheri Ren. (Pl. Iv). - Pallidissime lutescenti-viride, nitidum. Caulis 2-4 cent. altus, dense foliosus. Folia subsecunda, superne spiraliter contorta, 4 mill. longa, anguste lanceolata, summo apice denticulata, rete pallidissimo, cellulis internis obscuris, mediis ovalibus (diam $6 \mu$ ), basilaribus elongatis, in membranam scariosam conflatis, limbo hyalino lato $(40-60 \mu)$ e cellulis $15-25$-seriatis composito, papillis bifidis dorso valde prominentibus. Cætera ignota.

Madagascar: île Sainte-Marie, Perrot (Hb. Levier).

Espèce de la section Albescentia voisine du L. Isleanum Besch., des Seychelles, dont elle diffère par le margo 2 ou 3 fois plus large et par les papilles plus fortement saillantes.

L. (Syncratodictyon) REстuм Lac. - D'après un échantillon authentique provenant du Museum de Leyde et communiqué par $M$. le général Paris, il y a lieu de réunir cette plante au $L$. sinuosum Brid. des Mascareignes, comme forme à feuilles à peu près entières. Cette espèce, dont la présence dans le N.-O. de Madagascar avait été constatée avec doute, fait donc partie certainement de la Flore de cette île.

L. (Syncratodictyon) FUSCIF OLIUM Besch.

Madagascar: Forêt du versant oriental, Girọ Genet ; Fort Dauphin (Hb. Paris).

L. (Syncratodictyon) Garnieri Ren. Par. (Pl. xxiv). - Pallide viride. Cæspites molles. Caulis flexuosus parce divisus vel subsimplex, 2-4 cent. longus. Folia humida erecta subappressa, sicca plus minus secunda, apice flexuosa laxe subcrispatula, 4-5 mill. longa, late lanceolata, sensim angustata, breviuscule subulata, apice dentibus 2-3 coronata, alis undulatis tantum e medio involutis, inferne planis, summo apice dentibus nonnullis ornatis. Costa basi $52-54 \mu$ lata cum apice finiente. Auriculis excavatis hyalinis, e cellulis amplis rectangulis tenuiter limitatis efformatis. Limbo hyalino medium versus $18-24 \mu$, interdum paulo ultra lato e cellulis $6-8$ seriatis composito, superne angusto, quamvis usque sub apice continuo. Cellulis basilaribus breviusculis oblongis vel breviter rectangulis, internis quadratis, chlorophyllosis. Papillis densis dorso parum prominulis. Cætera desunt.

Madagascar: Jisy sur les racines des arbres, le long du ruisseau; "Pleurs des bœufs », SAvelli, 1904; province de Fianarantsoa, 1900, (Hb. Paris). 
Cette espèce est à peu près identique à une forme récoltée en très maigres échantillons dans la province de Fianarantsoa et que nous avions nommée $L$. Boivini Besch. var. brevifolium dans la Revue de 1900, p. 88. Elle est en effet très voisine du $L$. Boivini Besch.; cependant, bien qu'il n'y ait entre elles aucune différence anatomique importante, elle se distingue par un port différent, par les feuilles simplement flexueuses plutôt que cripulées à l'état sec, dressées à l'état humide, insensiblement rétrécies, plus brièvement cuspidées, tronquées au sommet, ondulées aux marges, planes (non condupliquées) dans la moitié inférieure.

\section{Gen. Campylopus.}

C. subcomatus Ren. Card.

Madagascar : Antseranandratsy (Hb. Paris).

C. pseudovirescens Ren. Card. - Dense cespitosus, lutescentiviridis, 2-3 cent. altus. Caulis erectus, rubro-tomentosus, plus minus divisus, superne parce et breviter ramosus vel subsimplex, densifolius. Folia conferta, rigidula, madida patentia, sicca erecta et apice caulis interdum vage subsecunda, e basi lanceolata, o,5-o,6 mill. lata, breviuscule subulata, canaliculata, 4-5 mill. longa, apice obtusiusculo dentata, marginibus superne remote et irregulariter serrulata, costa lata, plus quam tertiam partem basis occupante, superne plus minus longe excurrente, dorso breviter sed distincte lamelosa, superne dentibus scabra, in sectione transversali e 4 stratis cellularum composita : uno ventrali a cellulis majoribus, uno centrali ab eurycystis et duobus dorsalibus a cellulis minutis incrassatis formatis; cellulis laminæ minutis, irregularibus, nunc subquadratis vel transverse dilatatis, nunc oblique rhombeis vel breviter oblongis, inferioribus juxta costam majoribus et longioribus, alaribus quadratis, fuscis, auriculas magnas excavatas pulchre distinctas et totam basin laminæ occupantes efficientibus. Cætera ignota.

Madagascar: Maroantsetra, baie d'Antongil, sur un arbre pourri (Ch. Mathieu).

Par son port, ainsi que par la forme et le tissu des feuilles, cette espèce ressemble extrêmement au C. subvirescens Ren, et Card, également de Madagascar, mais elle en diffère essentiellement par la structure de sa nervure. Dans le C. subvirescens, une section transversale de cet organe montre avec un arc central d'eurycystes, des stéréides sur les deux faces; dans le $C$. pseudo-yirescens, on observe, au contraire, une couche ventrale de grandes cellules épidermiques, une couche d'eurycystes et deux couches dorsales de petites cellules à parois épaisses, qui ne sont cependant pas 
encore des stéréides et dont une partie se différencient pour former les lamelles. L'ábsence de vraies stéréides doit faire placer cette espèce dans le sous-genre pseudocampylopus, la structure de sa nervure étant à peu près identique à celle du C. subulatus Sch., telle que la représente Limpricht, Laubmoose, I, p. 385, fig. 125.

Une autre espèce malgache, le $C$. rigens Ren. et Card., qui offre aussi une grande ressemblance extérieure avec le $C$. pseudo-virescens, en diffère également par la struc. ture de sa nervure, qui présente sur la face ventrale une couche de cellules épidermiques plus petites que les eurycystes qu'elles recouvrent, et à parois épaissies, et des faisceaux de stéréides en dessous de l'arc d'eurycystes. Il est certain que la transition entre les vrais stéréides et les substéréides se fait graduellement, et, par conséquent, le caractère tiré de la présence ou de l'absence des stéréides dans une nervure peut parfois ne pas être d'un usage pratique. Mais, dans le cas présent, il n'est pas nécessaire de recourir à ce caractère pour distinguer le $C$. pseudo-virescens des deux espèces voisines : chez celles-ci, c'est la couche centrale d'eurycystes qui présente les éléments à plus large lumière, tandis que dans le $C$. pseudo-virescens, ce sont au contraire les cellules de l'épiderme ventral qui offrent le plus grand développement.

On voit par ce qui précède que, morphologiquement, cette espèce ne diffère pas des C.subvirescens et rigens; elle ne s'en distingue que par les détails de sa structure interne. Elle offre donc un remarquable exemple d'une espèce bien délimitée par ses seuls caractères anatomiques.

C. amblyacron Ren. Card. - Luride viridis. Cæspites densiusculi, complanati. Caulis brevis erectus 5 mill., rarius ro mill. altus. Folia mollia appressa, vix secunda, brevia 2 I/2 rarius 4 mill. longa, breviora subelliptica brevissime acuminata,longiora costa melius excedente paulo longius acuminata; marginibus planis integris tantum apice obtuse eroso-crenulatis. Costa vix $\mathrm{r} / 3$ saepius $\mathrm{r} / 4$ folii latitudinis occupante, dorso mediocriter lamellosa lævi, tantum summo apice breviter, passim obtuse excedente, remote serrata, in sectione transversali e cellulis 3-4 stratosis composita quarum strato ventrali e cellulis hyalinis laxis quadrangulis formato, stereidis dorsalibus $2-3$ stratosis. Cellulis basilaribus juxtacostalibus rectangulis, marginalibus 8-1o seriatis multo minoribus, quadratis, sequentibus inæqualibus, irregularibus oblique rhombeis, passim ovalibus, alaribus dilatatis, hyalinis hexagonis auriculas excavatas efformantibus. Cætera desunt.

$$
\text { Madagascar: Ankeranadinika, Com DorR, } 1896 \text {. }
$$

Cette espèce que nous avions d'abord rattachée comme var. amblyacron au $C$. Cambouei R. C. doit en être séparée à cause de la contexture du tissu basilaire dont les cellules marginales, sur une large bande 8-12 sériée sont petites, courtes, carrées, tandis que dans le C. Cambouei ces cellules sont étroites et allongées, comme d'ailleurs dans la plupart des Campylopus piliferes. La nervure comprend 2 couches d'eury'cystes l'une épidermique ventrale à cellules très dilatées, l'autre contiguë à l'in- 
térieur formée de cellules moins grandes, puis, sur la face dorsale, des substéréides en plusieurs couches entremêlées de stéréides. Les lamelles dorsales sont peu élevées et formées d'une seule cellule.

Le dimorphisme des feuilles, celles des tiges courtes subelliptiques très brièvement acuminées à pointe obtusiuscule épaisse due à la brève excurrence de la nervure, celles des tiges plus élevées oblongues acuminées et du double plus longues, ce dimorphisme n'a pas d'analogue dans d'autres espèces de la région. Toutefois cette plante appelle de nouvelles observations avant d'être reconnue comme bonne espèce.

C. Verdolini Par. Ren. - Laxe cæspitosus. Caulis erectus 3-4 cent. altus, inferne mediocriter tomentosus, superne fasciculato-ramosus, innovationes imbricate foliosas emittens. Folia inferiora subsecunda 4-6 mill. longa, superiora plus minus imbricata, comalia interdum congesta, flexuosa, omnia e medio vel infra involuta, subulata passim apice truncatula, marginibus superne serratis, costa $2 / 3$ folii latitudinem occupante dorso rotundate lamellosa, superne remote serrata, sectione transversali modo sequente composita: $I^{\circ}$ pagina ventrali, e cellulis magnis, hyalinis, bi-stratosis ; $2^{\circ}$ dorso, e cellulis vacuis medie magnis alternantibus; $3^{\circ} \mathrm{e}$ cellulis stereidiformibus inter præcedentes interrupte aggregatis. Cellulis folii mediis rhombeis secus margines minoribus, quadratis usque basin versus descendentibus, alaribus magnis cruentofuscescentibus auriculas maxime excavatas sistentibus. Fructus in eodem perichætio 3-4. Capsula in pedicello I o- I m mill. longo arcuato, flexuoso demum erecto pallido, humida anguste oblongo-piriformis collo attenuato elongato, sicca arcuata anguste cylindrica, sulcata, pallide castanea, collo obsolete strumoso. Calyptra basi fimbriata pro more in perichætio apice nidulans nec non radicans. Cætera desunt.

$$
\text { Madagascar: vallée de l'Ofika, Verdolin, igo I (Hb. Paris). }
$$

Voisin par le port du C. Heribaudi R. C. dont il diffère par les lamelles dorsales de la nervure, obtuses et peu saillantes, formées d'une seule cellule arrondie et non de crêtes élevées pluricellulaires, par la subule moins fortement dentée, par le pédicelle plus long et la capsule étroitement piriforme atténuée en un long col peu ou point renflé. La structure de la nervure est à peu près la même que dans le C.Gallienii Par., mais ce dernier se distingue à première vue par un port plus robuste, par les feuilles uniformément secondes et plus grandes, par la subule plus fortement dentée et par l'absence d'innovations, à feuilles dressées appliquées.

C. Gallienii Par. (Pl. vi). - Cæespites laxi, inferne fuscescentes, superne saturate virides. Caulis $4-6$ cent. longus e basi prostrata ascendens, subsimplex, tomento rufo usque ad apicem obtectus. Folia remotiuscula laxe hamato-secunda, haud in comam congesta, 6-8 mill. 
longa lanceolato-subulata, marginibus e medio et infra involutis, superne convolutaceis et argute serratis, summo dentibus plerumque geminatis ; costa lata (usque 0,4 mill.) dimidium fere folii latitudinis basi occupante, dorso lamellosa et superne dentata. Cellulis alaribus amplis, rufescentibus, auriculas magnas excavatas efformantibus, basilaribus juxta costam, magnis rectangulis, sequentibus elongate rhombeis apicibus attenuatis obtusis, fere recte seriatis, parietibus interrupte sinuosis, mediis et superioribus juxta costam rhombeis oblique seriatis marginalibus in tota folii longitudine subito brevioribus quadratis vel breviter rectangulis ascendendo minoribus. Cætera desunt.

Madagascar: cercle de Fort Dauphin à Manantantely, Igoo (Hb. Paris).

Grande et belle espèce d'un vert d'émeraude qui se distingue à première vue des C. comatus R. C. et subcomatus R. C. par un port plus robuste scoparioïde, par les feuilles falciformes non réunies en touffes au sommet des innovations et ne formant pas d'étages successifs, enfin par la nervure plus large. Celle-ci est composée de 2 couches d'eurycystes à très grande lumière l'une en épiderme ventral, l'autre contiguë centrale, puis sur la face dorsale, des groupes de stéréides. Les lamelles sont arrondies, peu élevées et formées d'une seule cellule.

B. filescens Ren. Card.

Madagascar: province d'Ámbositra, Frissard (Hb. Paris).

\section{TRICHOPHYLLA}

C. Arbogastr Ren. Card, var. capitulifer Ren. Card. - A forma typica differt habitu paulo robustiore, colore nigro-viridi et caulibus apice abrupte capitulato-clavatis.

Madagascar: Maroantsetra, baie d'Antongil, sur un arbre pourri; Mathieu (Hb. Cardot).

C. Orzeszkoanus Ren. Card. (Pl. Ix et x). - Cespites densiusculi, parum cohærentes, superne laete vel salurate virides, intus fuscescentes, terra obruti. Caulis erectus, $2-3$ centim. altus, basi tomentosus, subsimplex, apice attenuatus, acutus. Folia erecto-patentia, rigidiuscula, superiora subimbricata, plerumque epilosa, nonnulla comalia breviter pilifera, 5 mill. longa, inferne plana, mox involuta, apice convolutacea, integra vel summo dentibus remotis paucis instructa, costa lata, $1 / 3-1 / 2$ 
vel ultra folii latitudinem basis occupante, dorso laevi, haud lamellosa, in sectione transversali e strato medio cellularum magnarum vacuarum et stereidis ventralibus et dorsalibus pluristratosis composita, cellulis laminae mediis elongate rhombeis vel sublinearibus, flexuosis, oblique seriatis, basilaribus omnibus pallide viridibus, rectangulis, subrectangulis vel elongate subhexagonis, marginalibus inferioribus perangustis, 6-1 o seriatis, alaribus rufo-aurantiis, auriculas excavatas efformantibus. Cætera desunt.

Grande Comore: sur la terre, entre les pierres, près du sommet du volcan Kortala, alt. 2000-230o m., MAcÉ (Hb. de Poli).

Cette espèce que nous dédions à notre ami M.N.Orzeszko, connu par ses savants travaux sur l'histotaxie des Graminées, appartient à la section Palinocraspis Lindb. et est voisine du $C$. crateris Besch. de la Réunion. Elle en diffère par ses touffes vertes, denses, par les feuilles beaucoup plus courtes, simplement hyalines à la pointe, les comales seules parfois brièvement pilifères, par les cellules suprabasilaires verdâtres, lâches, non poreuses-dicranoïdes comme dans l'espèce voisine. La structure de la nervure est la même, et comprend un arc central d'eurycystes, complètement recouvert sur la face dorsale par la zone des stéréides, tandis qu'il ne l'est que partiellement sur la face ventrale, de telle sorte que les cellules de chacune des extrémités de l'arc affleurent en épiderme de chaque côté de la zone ventrale des stéréides.

\section{Cambouei Ren. Card.}

Cette plante figure dans le Prodrome, dans la section Trichophylla et une note rectificative insérée aux Addenda la place dans la section Atricha. Un nouvel examen de cette plante m'a fait revenir, malgré l'absence de poil, à la première opinion. Si l'on considère l'ensemble nombreux des Campylopus de notre Domaine (et il est probable qu'on pourrait donner beaucoup plus de généralité aux observations suivantes), on remarque 2 types de tissu foliaire qui se retrouvent bien distincts, sinon dans la totalité, du moins dans la grande majorité des espèces selon qu'elles appartiennent à la première ou à la seconde de ces sections.

Dans la section Trichophylla le tissu basilaire est généralement hyalin avec des cellules marginales qui s'allongent beaucoup en devenant très étroites; les cellules moyennes à parois très épaisses montrent un lumen linéaire ou du moins allongé, flexueux et descendent ordinairement en coin dans le tissu délicat de la base. Les cellules alaires peuvent former ou non des oreillettes excavées quelquefois colorées mais plus souvent hyalines, mais même dans les espèces qui en possèdent, elles ne sont pas toujours développées également dans toutes les feuilles d'un même échantillon, à ce point qu'il faut examiner un certain nombre de feuilles pour être assuré que la plante est munie ou dépourvue d'oreillettes.

Dans la section Atricha le tissu est généralement chlorophylleux dans toute son étendue, seulement plus pâle à la base, les parois restant fermes, les cellules basilaires juxtacostales, plus ou moins régulièrement rectangulaires et assez grandes, 
deviennent plus petites en s'avançant vers les marges où elles prennent la forme carrée en dessinant une bande marginale 6-12 sériée. Les cellules moyennes sont régulièrement rhombées en séries obliques passant vers les marges à une bande plus ou moins large de cellules plus petites, carrées, en séries droites. Dans quelques espèces les oreillettes manquent; dans le plus grand nombre elles sont très développées, presque toujours franchement colorées, fortement excavées et n'offrent pas la variabilité qu'on remarque dans le type précédent (Trichophylla).

Dans quelques espèces des Atricha, notamment dans le groupe des $C$. virescens Besch., C. subyirescens R. C., C. rigens R.C., et C. pseudo virescens R.C., ce type de tissu se modifie un peu en ce sens que les cellules de la moitié inférieure de la feuille sont rectangulaires en séries droites, et c'est seulement dans la partie supérieure de la feuille que les cellules deviennent rhombées ou irrégulî̀res.

Je ne parle pas ici de quelques autres modes de tissu foliaire qui n’appartiennent qu'à un nombre relativement restreint d'espèces.

Dans les Trichophylla le poil manque quelquefois aux feuilles moyennes et même aux comales chez des espèces à feuilles normalement pilifères. Ce fait se produit, comme on le sait, dans le C. polytrichoĩdes de Not. d'Europe. Lorsqu'on se trouve en présence d'un cas douteux entre les deux sections, par exemple dans celui du C. Cambouei R. C., la forme des cellules basilaires marginales semble être le meilleur caractère à utiliser pour le classement.

L'épaississement irrégulier des parois des cellules moyennes dans la section Trichophylla, ce qui donne au lumen une forme allongée, linéaire ou sublinéaire flexueuse tient probablement à ce que les espèces de cette section croissent généralement dans des lieux découverts où elles sont exposées à une vive lumière et à des alternatives d'humidité et de dessèchement, tandis que la majeure partie des espèces de la section Atricha végètent plutôt sur l'humus des forêts.

Il y aura lieu d'observer si quelques espèces ou formes de la section Atricha en croissant dans des lieux découverts à période sèche prolongée, ne se modifient pas par l'épaissement des parois des cellules foliaires et l'irrégularité de la forme du lumen qui en est la conséquence. J'ai constaté déjà ce fait sur 2 formes du plateau central de l'Imerina qui ont la plus grande analogie avec $C$. Cambouei R. C., sauf la forme carrée des cellules basilaires marginales, ce qui, avec l'absence de poil empêche de les classer dans les Trichophylla et rend probable leur autonomie spécifique.

La structure de la nervure des Campylopus, qui a été décrite d'excellente façon par Limpricht offre de précieux caractères pour la délimitation de beaucoup d'espèces. On y distingue des types principaux auxquels se rattachent la majeure partie des espèces. On peut donc former ainsi des groupes d'espèces du même type de nervure (saufquelques particularités de détail servant aux différenciations spécifiques); mais si l'on se base sur ces types de structure pour fonder des sous-genres, on se heurte à la même difficulté qni m’a arrêté dans l'établissement de coupes subgénériques dans les Dicranoloma, et cela pour le même motif, je veux dire le manque d'une concordance suffisante entre les divers modes de structure de la nervure et les autres caractères morphologiques ou histologiques.

Tout d'abord la distinction entre les cellules à petite ou moyenne lumière et les substéréides, d'autre part entre celles-ci et les stéréides, est souvent subtile; les unes passent aux autres insensiblement et l'épaississement des parois cellulaires peut dépendre des conditions biologiques dans lesquelles se développe tel individu plutôt 
que du fait d'une structure primordiale caractérisant l'espèce. En second lieu, et cette observation s'applique surtout à la section Trichophylla, des espèces extrêmement affines aussi bien par leurs caractères morphologiques que par la texture de leur tissu foliaire, n'ont pas la même structure de nervure. Dans le plus grand nombre on retrouve le type ordinaire des Eucampylopus avec Io un arc d'eurycystes en épiderme ventral; 20 un arc central d'eurycystes contigu au précédent; $3^{\circ}$ des groupes de stéréides sur la face dorsale; mais quelques espèces de la section Trichophylla par ex. C. crateris Besch., C. Orzeszikoanus Ren. Card, pour ne parler que de notre Domaine, sont du type Palinocraspis Lindb. avec un arc central d'eurycystes affleurant plus ou moins en épiderme ventral à ses deux extrémités seulement et des stéréides sur les deux faces ventrale et dorsale.

En présence de ces contradictions, il ne semble donc pas utile de fonder des sous-genres basés sur la structure de la nervure, tandis que les sections telles qu'elles figurent dans les Pflanzen Familien de M. Brotherus, d'après un ensemble de caractères, correspondent au contraire à des groupes très naturels d'espèces.

C. Parisii Ren. - Humilis, dense cæspitosus, saturate viridis. Caulis erectus 6-8 mill. altus sæpius basi denudatus, albido tomentosus. Folia dense conferta, stricta, appressa circa 2 mill. longa, lanceolata inferiora apice hyalina, comalia breviter pilifera (pilum subintegrum vel-dentibus nonnullis remotis instructum) marginibus jam fere e basi late involutis, integris. Cellulis basilaribus pallidis quadratis ve! brevissime rectangulis, marginalibus elongatis angustis hyalinis, mediis oblique rhombeis, passim ad angulos papilliformi prominentibus, alaribus hyalinis, passim dilatatis auriculas sæpe parum conspicuas efformantibus. Costa $2 / 5$ basis latitudinem occupante (o,20 mill. lata) superne dorso vix rugulosa, in sectione transversali dorso haud lamellosa, tantum undulata. Duces unistratosi in pagina ventrali, cellulis mediis et dorsalibus minutis stereidiformibus.

Madagascar: province d'Ambositra, Salvan ( $\mathrm{Hb}$. Paris). Dédié à M. le général Paris qui par son infatigable activité a beaucoup contribué au progrès de la Bryologie de Madagascar.

Par le port, cette espèce se rapproche beaucoup du C. Caillea R. C. de Nossi Bé, mais elle s'en distingue par ses marges nọ dentées sous la pointe hyaline, par la nervure relativement plus large et les cellules moyennes régulièrement rhombées plus courtes.

D'autre part elle differe du C. calvus R. C. parles feuilles de moitié plus courtes, les comales nettement pilifères à poil faiblement denté, par les cellules alaires dilatées formant parfois des oreillettes, enfin par les cellules moyennes régulièrement rhombées, courtes, à parois non épaissies, et non allongées flexueuses, irrégulières. La nervure se compose d'un arc d'eurycystes en épiderme ventral, au-dessous duquel se trouvent des groupes de cellules stéréidiformes. Les lamelles sont faiblement indiquées par une seule cellule elliptique faisant saillie seulement dans la moitié de sa hauteur. 


\section{Fam. LEUCOBRYACE. 平}

Tout ce qui concerne cette Famille est exposé textuellement d'après les remarquables travaux de M. Cardot, notamment d'après les «Leucobryacées de Madagascar" publiées dans le Bulletin de l'Herbier Boissier, $2^{\mathrm{e}}$ série, tome IV (I904). Je renvoie à cet excellent mémoire pour les explications complémentaires et les notes critiques qui ne peuvent être reproduites entièrement ici.

\section{Tribu I. - LEUCOBRYEA Card.}

Nervure sans faisceau scléreux. Chlorocystes de section quadrangulaire en une seule assise dans toute la longueur de la nervure et placées au point de jonction de quatre leucocystes.

\section{Gen. 0chrobryum Mitt.}

Nervure déprimée convave, canaliculée, sans faisceau scléreux, homostrosique. Leucocystes toujours en 2 couches (sauf vers la pointe de la feuille dans certaines espèces). Ailes étroites disparaissant vers le sommet où elles sont quelquefois remplacées par un margo formé de plusieurs cellules un peu épaissies. Tige dépourvue de faisceau axile. Fructification terminale. Capsule hémisphérique, cyathiforme après la chute de l'opercule, très brièvement immergée dans le perichèze ; opercule conique rostré tombant avec la coiffe, celle-ci étroitement conique, longuement subulée, fimbriée à la base. Péristome nul.

O. sakalavum Card. et Par. - Minimum, cespitulosum, glaucoviride, 2,5-6 mill. altum, interdum subacaule. Folia patentia, subflexuosa curvatulave, lineari-lanceolata, longe acuminata, 2,4-2,6 mill. longa, o,3-o, 4 lata, apice obtusiusculo minute apiculato subacutove, integerrima, tota fere longitudine canaliculata, summo apice tamen e sectione subtrigonali vel semicirculari, alis angustis, medium versus evanescentibus, inferne a 5-7 seriebus cellularum elongate rectangularum margines versus linearium compositis; costa perfecte homostrosica, chlorocystis in costæ majore parte subcentralibus vel leviter hypercen- 
tralibus, superne distincte hypercentralibus, leucocystis elongate rectangulis, subhexagonis vel subquadratis. Folia perichætialia aliquanto latiora, alis latioribus a 7-12 seriebus, cellularum compositis. Capsula in pedicello pallido, cum vaginula fusca 1,25 mill. alto, foliis perichætialibus superata, minima, cyathiformis, pallide fusca, leptoderma, o,3$0,4 \mathrm{mil}$. longa, o,3 mil. ore lala, operculo convexo-conico, recte rostrato, I mil. alto, unacum calyptra straminea, longe subulata, basi fimbriata, 2-5-2,75 mil. longa, folia perichætialia superante, secedente.

Madagascar: territoire sakalave, cercle de Maintirano, secteur de Rakobe, poste d'Ampoza, près du village de Tahutofoly, sur bois pourri; récolté par un tirailleur sakalave (Hb. G. Paris).

Beaucoup plus petit que $O$. Normandi Card. Par. de l'Afrique occidentale, dont il differe en outre par les feuilles ordinairement apiculées et par les cellules inférieures des ailes moins étroites.

En l'absence de fructification, les $O$. Boivini Besch. et O.Rutenbergii C. M., à cause de la structure basilaire de leur nervure, doivent être placés de préférence dans le genre Leucobryum.

\section{Gen. Leucobryum Hpe.}

Nervure déprimée concave, canaliculée et souvent subtubuleuse dans le haut, sans faisceau scléreux, homostrosique ou hétérostrosique. Ailes généralement étroites ou assez larges, disparaissant vers le sommet ou réduites à une ou deux rangées de cellules. Pas de margo distinct. - Tige pourvue ou non d'un faisceau axile. Fructification terminale ou latérale. Capsule asymétrique arquée, souvent strumeuse, longuement pédicellée. Coiffe dimidiée. Péristome très développé dicranoïde.

Groupe I. - Nervure hétérostrosique (rarement subhétérostrosique); 3 à 6 couches de leucocystes dans la partie basilaire, de chaque côté de la ligne médiane, 2 dans le reste de la nervure.

\section{Boryanum Besch.}

M. Cardot a reconnu que les L. Hildebrandtii C. M., L. Rutenbergii (C. M.) Besch. et L.lave Mitt. (ex parte) ne sont pas distincts spécifiquement du L. Boryanum Besch. Les localités de Madagascar citées pour ces 3 plantes doivent en conséquence être attribuées au L. Boryanum Besch. Il faut y ajouter: vallées du Lohato et de l'Ofika (Verdolin, Hb. Paris). 
L. Perroti Ren. Card.

Un échantillon de l'herbier de Kew, communiqué à $M$. Cardot sous le nom de L. lave Mitt., Madagascar (Dr Meller), doit être rapporté au L. Perroti R. C. Il en est de même d'un échantillon communiqué par l'abbé Boulay sous le nom de $L$. irregulare Besch. in litt. Ce specimen récolté par Bernier provient vraisemblablement de Madagascar.

L. pseudo-madagassum Card. in Bull. Hb. Boissier. - Cespites robusti, densissimi, albescentes vel pallidissime lutescentes, 3-4 cent. alti. Folia conferta, dense erecto-imbricata, interdum subhomomalla, oblongo-lanceolata, breviuscule vel longe acuminata, 4-7,5 mil. longa, I,25-I, 5 lata, superne canaliculata, apice acuto apiculatove integra vel parce et minutissime denticulata, cæterum integerrima et lævia, alis latitudine variabili, usque fere ad apicem angustissime productis, inferne e 6-1 5 seriebus cellularum formatis, cellulis inferioribus quadratis vel subquadratis, sequentibus elongate rectangulis, marginalibus linearibus ; costa heterostrôsica, leucocystis oblongo-hexagonis, inferne in parte mediana bi-vel tristratosis, lateraliter in 4 stratis, quorum 2 ventralibus et 2 dorsalibus, deinde usque ad apicem bistratosis, chlorocystis inferne centralibus vel subcentralibus, in parte superiore distincte hypocentralibus. Capsula in pedicello purpureo, $7-8$ mil. longo, horizontalis, valde arcuata, basi strumosa, sicca sulcata; operculo ignoto. Peristomium purpureum, elatum.

Madagascar : Imerina, Andrangoloaka, Hildebrandt ; Betsileo, sine loco, Rev. Montaut, 1894 .

Par son port, cette espèce rappelle d'une façon frappante le L. madagassum Besch. avec lequel on l'a confondue: elle est cependant un peu moins robuste et en differe d'ailleurs essentiellement par la structure de sa nervure. Les caractères anatomiques, notamment les chlorocystes hypocentriques dans le haut de la nervure, la rapprochent du $L$. Guienżii C. M. du Transvaal et du Natal; mais d'après le seul échantillon examiné ( ${ }^{\circ}{ }^{4} 54$ de Rehmann), ce dernier est beaucoup moins robuste et a les feuilles plus petites, moins imbriquées et bien plus étroites, avec un port très différent.

\section{Isleanum Besch.}

“ Cette espèce rappelle assez le L. candidum (Brid.) Jaeg. de la Nouvelle Zélande; elle est toutefois moins robuste que celui-ci et s'en distingue surtout par ses feuilles lisses sur le dos et moins épaisses à la base. La nervure est tantôt franchement hétérostrosique avec 4 assises de leucocystes à la base de chaque côté de la partie médiane qui n'en a que 2, tantôt seulement subhétérostrosique ou même subhomostrosique 
d'un côté, les assises supplémentaires étant plus ou moins incomplètes. Les chloro. cystes souvent hypercentriques dans le bas, à peu près centriques dans la partie moyenne, deviennent en général assez nettement hypocentriques vers le sommet. Les ailes de largeur très variable sont formées de 6 à 14 séries de cellules. Les feuilles presque toujours un peu courbées et homotropes mesurent de 4 à 5,5 mil. de long sur 0,75 à 1 mil. de large. Le faisceau axile que l'on observe dans la tige des espèces précédentes, fait généralement défaut ou est très peu distinct dans le $L$. Isleanum, et les cellules corticales sont à peines différenciées. ఎ

M. Cardot considère le L. molle C. Müll. (Ren., Prodrome, p. 107) comme une var. molle (C. M.) Card. du L. Isleanum Besch. différant du typepar ses dimensions plus faibles, ses feuilles plus petites, longues de 2,65 à 3,5 mil. et larges de 0,5 à 0,8 mil., mais elle se relie au type par toute une série de formes intermédiaires.

En conséquence il y a lieu de rattacher au type du L. Isleanum les plantes des localités malgaches suivantes : île Sainte-Marie, Andevorante, Fénérive; forêt de Mahambo (Perrot); Imerina : forêt d'Amperifery (Rev. Campenon), Ambatomanga (Rev. Talazac), Antsachambavy (Dalincourt), et d'attribuer à la var. molle (C.M.) Card. les localités citées dans le Prodrome, ne faisant pas double emploi avec les précédentes, en y ajoutant : district de Moramanga, vallée de l'Anamalazaotra (Carron, Hb. Paris); Forêt de Fito, district de Tamatave (Perrot, Hb. Levier); vallée du Lohato (Verdolin, Hb. Paris) ; Maroantsetra, baie d'Antongil (Mathieu).

D'après l'examen d'un échantillon original reçu de C. Müller lui-même de $L$. reticulatum C. M. mss. (Imerina, leg. Hildebrandt), M. Cardot conclut que cette plante ne diffère en rien de L. molle C. M.

L. acutifolium Card in Bull. Hb. Boissier. (Schistomitrium acutifolium Mitt, in Journ. Linn. Soc., pro parte).- Cespites humiles albescentes. Folia patentia vel homomalla, e basi lanceolata longe acuminata, 3,5-4,5 mil. longa, o,6-o,8 lata, superne valde canaliculata, in apiculum elongatum acutissimum desinentia lævia et integerrima, alis latiusculis, superne evanescentibus, inferne e 5-8 seriebus cellularum formatis, cellulis inferioribus subquadratis vel breviter rectangulis sequentibus elongate rectangulis, marginalibus superioribusque anguste linearibus; costa nunc heterostrôsica, nunc subheterostrôsica, vel quoque uno latere heterostrôsica, altero subhomostrôsica, leucocystis oblongo-hexagonis vel subrectangulis, inferne plus minus 3-4 stratosis, chlorocystis basin versus leviter hypercentralibus, cæterum usque ad apicem centralibus vel subcentralibus. Cætera ignota.

Madagascar: Tananarive, $\mathrm{D}^{\mathrm{r}} \operatorname{Meller}(\mathrm{Hb}$. Kew).

a Par le port, la taille, la forme des feuille et la structure de la nervure à la base, cette espèce se rapproche beaucoup du L. Isleanum Besch. var. molle Card.; elle s'en distingue par ses feuilles pourvues d'un apicule plus long et plus aigu et par ses chlorocystes à peu près centriques jusqu'à la pointe. 
L. cucullatum Broth., var. Rutenbergii Card. in Bull. Hb. Boissier (Ochrobryum (?) Rutenbergii C. Müll. in Reliq. Rutenb.).

Madagascar: dans la forêt Ambaranavarana, Rutenberg (Hb. Museum Berlin); district de Majunga, Mampikony, Savelli (Hb. Paris).

" Diffère du L. cucullatum Broth. de l'Usambara par ses feuilles plus petites, plus courtes (long. r,75-2 mil., larg. 0,5 à 0,6 mil. au lieu de 2,5 sur 0,7 à 0,8 mil.), plus obtuses, à peine apiculées. Les caractères anatomiques concordent entièrement. La nervure est hétérostrosique; leucocystes en 4 couches dans le bas $(2$ dorsales et 2 ventrales) de chaque côté de la partie médiane, formée seulement de 2 couches; chiorocystes assez distinctement hypercentriques dans toute la longueur de la nervure, les supérieures très courtes en section longitudinale. Ailes étroites formées dans le bas de 3 à 6 séries de cellules. Faisceau axile et cellules corticales de la tige plus ou moins différenciées. Plante de petite taille formant des touffes denses d'un vert glauque ou d'un blanc jaunâtre. Fructification inconnue."

Groupe II. - Nervure hétérostrosique; 3 là 6 couches de leucocystes dans la partie basilaire et dans la partie supérieure de la nervure.

L. COMOREnse C. Müll.

Madagascar : Vohémar, Perrot.

Maurice: Rev. Rodriguez.

L. Sanctæ Mariæ Card. in Bull. Hb. Boissier. - Cespites densi, albescentes, I-3 cent. alti. Folia sat conferta, erecto-patentia, sæpe homomalla, sicca rigidula, anguste lanceolata, sensim longe acuminatosubulata, 3,5-5,5 mil. longa, 0,5-0,65 lata, superne valde canaliculata, apice obtusiusculo apiculato, lævia et integerrima, alis latitudine variabili, superne evanidis, inferne e 3-7 seriebus cellularum formatis, cellulis inferioribus breviter rectangulis quadratisve, sequentibus elongate rectangulis, marginalibus superioribusque anguste linearibus; costa heterostrôsica, Ieucocystis rectangulis vel oblongo-hexagonis, inferne in parte mediana bistratrosis et lateraliter partim 3-4-stratosis, deinde bistratosis, denique superne iterum plus minus regulariter 3-4-stratosis, chlorocystis fere ubique subcentralibus, tantum apice pro more leviter hypocentralibus. Cætera ignota.

Madagascar : île Sainte-Marie (Ed. MARIE in Hb. Bescherelle); Rev. ARBOGast, I890.

* Cette espèce, qui a été distribuée par Bescherelle sous le nom de $L$. comorense C. Müll., s'en rapproche en effet par la taille, le port et les caractères anatomiques. 
Mais l'espèce de C. Müller a les feuilles beaucoup plus larges à la base, assez brusquement contractées pour former l'acumen, moins raides à l'état sec et généralement plus homotropes; en outre, la nervure est plus épaisse dans le bas, où l'on trouve normalement cinq ou six couches de leucocystes et où les chlorocystes sont hypercentriques. Je dois dire cependant qu'une Mousse récoltée à Maurice par le Rév. Rodriguez et que je rapporte au $L$. comorense, bien qu'ayant la forme de feuilles de celui-ci, présente plutôt la section basilaire du Sancta Maria; il est donc probable que ce dernier caractère n'est pas absolument constant, et c'est surtout par la. forme des feuilles que l'on pourra distinguer l'une de l'autre ces deux espèces. "

L. MAdaGassum Besch.

Madagascar: mont Antely près d'Ambositra, Forsyth Major (Hb. Levier); province de Betafo, Galinon (Hb. Paris).

Groupe III. - Nervure homostrosique, rarement subhétérostrosique, jamais franchement hétérostrosique; leucocystes le plus souvent en 2 couches dans toute la longueur de la nervure.

L. mayottense Card. in Bull. Hb. Boissier. - Cespites densi, pallide lutescentes vel glauco-virides, I-3 cent. alti. Folia conferta, erecta, superiora interdum homomalla, e basi oblonga longe acuminata, $2,1-3,75$ mil. longa, $0,6-0,75$ lata, superne canaliculata, apice acuta vel apiculata, lævia et integerrima, alis latiusculis superne evanescentibus, inferne e 5-7 seriebus cellularum formatis, cellulis inferioribus quadratis breviter rectangulis, marginalibus superioribusque linearibus; costa subhomostrôsica vel subheterostrôsica, leucocystis brevibus plerisque subquadratis vel hexagonis, fere ubique bistratosis, basi tantum hic illic tristratosis (strato supplementari dorsali pro more valde imperfecto), chlorocystis ubique subcentralibus. Cætera desunt.

Comores: Mayotte, Boivin; Grande-Comore au mont Kortala, Macé, 1900 (Hb. de Poli).

« Bien que la fructification de cette Mousse soit encore inconnue, il paraît cependant certain qu'elle appartient au genre Leucobryum, et non au genre Ochrobrymm, comme le pensait Bescherelle. Il est à remarquer, en effet, que tous les Ochrobryum indiscutables, c'est-à-dire tous ceux dont la fructification est connue, ne présentent jamais que deux couches de leucocystes à la base de la nervure, sans aucune trace de dédoublement de.l'une ou de l'autre assise. Tel n'est pas le cas pour l'O. Boivini Besch., chez lequel on observe presque toujours le dédoublement d'un certain nombre des leucocystes inférieures de l'assise dorsale, comme cela a lieu chez beaucoup de Leucobryum, et qui, par suite, me semble devoir être placé de préférence dans ce dernier genre. » 
L. parvulum Card. in Bull., Hb. Boissier. - Minimum, tenellum, albescens, o,5-1 cent, altum. Folia erecta vel erecto-patentia, e basi oblonga vel lanceolata longe acuminata, 2,25-3,25 mil. longa, o,5-o,7 lata, superne canaliculata, apice acuto, lævia et integerrima, alis latiusculis superne evanescentibus, inferne e 5-1o seriebus cellularum formatis, cellulis elongatis rectangulis, marginalibus superioribusque linearibus; costa homostrôsica vel subhomostrôsica, leucocystis plerisque rectangulis vel oblongo-hexagonalibus, ubique vel fere ubique bistratosis, interdum basi hic illic tristratosis, chlorocystis ubique centralibus vel subcentralibus. Cætera ignota.

Madagascar: sine loco (Borgen; Hb. Kew ; Hb. G. Paris); île Sainte-Marie (Perrot frères; Hb. E. Levier) ; vallée de l'Ofika (Verdolin; Hb. G. Paris); Imerina: Ankadivavala et entre Analamazoatra et Andevorante (Rev. Camboué).

"Cette petite forme est certainement bien voisine du $L$. mayottense Card., dont elle n'est peut-être qu'un état appauvri. Cependant, le tissu présente quelques différences, assez légères à la vérité, mais qui m'ont paru constantes, ce qui m’a décidé à la décrire comme espèce distincte. Ces différences consistent en ce que, dans le L. parvulum, la plupart des leucocystes de la nervure sont au moins une fois plus longues que larges, et que les cellules des ailes ont la forme de rectangles allongés, tandis que,dans le L. mayottense, le plus grand nombre des leucocystes de la nervure sont carrées ou très brièvement rectangulaires, ou encore subhexagonales et à peine plus longues que larges, et le tissu des ailes est plus lâche, formé de cellules carrées ou brièvement rectangulaires $\gg$.

L. Galinoni Card. Par. in Rev, bryol. (nomen solum), et in Bull. Hb. Boissier. - Tenellum, humile, dense cespitosum, albescens vel glauco-viride, I-2 cent. altum. Folia patentia vel erecto-patentia, e basi anguste lanceolata longe acuminata, 2,5-3,35 mil. longa, o,5-o,6 lata, superne canaliculata, apice obtuse abrupte minute apiculata, lævia et integerrima, alis latiusculis, superne evanidis, inferne e $5-8$ seriebus cellularum formatis, cellulis rectangulis, marginalibus superioribusque linearibus; costa homostrôsica vel subhomostrôsica, nonnunquam subheterostrôsica, leucocystis oblongo-hexagonis, parietibus internis longitudinalibus poris minimis perforatis, nunc ubique bistratosis, nunc inferne plus minus tristratosis, chlorocystis ubique centralibus, subcentralibus vel inferne hypercentralibus. Cætera desiderantur.

Madagascar: province de Betafo, rive droite de la Mania (Galinon; Hb. G. Paris); cercle de Maintirano, poste de Dembavy (Regouby; Hb. G. Paris). 
"Cette espèce se rapproche des deux précédentes par ses petites dimensions et ses caractères anatomiques; elle s'en distingue principalement par ses feuilles obtuses, brusquement apiculées, et par les parois internes longitudinales des leucocystes percées de pores beaucoup plus petits; les pores des parois transversales sont plus grands, et à peu près de même dimension que ceux des deux espèces voisines. "

\section{Tribu II. - LEUCOPHANEA Card.}

Nervure pourvue d'un faisceau scléreux (Stéréome). Chlorocystes de section quadrangulaire, en une seule assise dans toute la longueur de la nervure et placées au point de jonction de 4 leucocystes.

\section{Gen. Leucophanes Brid.}

Nervure déprimée, canaliculée ou pliée en gouttière sur la plus grande partie de sa longueur, homostrosique ou hétérostrosique, pourvue d'un faisceau médian scléreux (stéréome) formé de stéréides ou de substéréides, émergeant ordinairement sur la face dorsale, parfois recouvert de ce côté, surtout dans la partie supérieure de la feuille, par la couche dorsale de leucocystes, généralement dilaté et denté, papilleux, tuberculeux ou propagulifère sur le dos vers le sommet. Ailes de largeur variable et souvent inégale, disparaissant toujours loin du sommet. Margo bien distinct, composé de cellules très étroites, sclérifiées et pluristratifiées dans la partie supérieure de la feuille, formant ainsi 2 stéréomes marginaux. Tige dépourvue de faisceau axile. Fructification terminale ou latérale. Capsule symétrique, oblongue ou subcylindrique, dressée, longuement pédicellée. Coiffe dimidiée. Péristome non dicranoïde.

L. mayottense Card. in Bull. Hb. Boissier. - Pallide lutescens albo-viride, fragile, dense cespitosum, I,5-2 cent. altum. Folia sat conferta, erecto-patentia, anguste lineari-lanceolata et longe loriformiacuminata, 6-7 mil. longa, 0,4-0,7 lata, plicato-canaliculata, apicem versus deplanata, acuta obtusulave, alis latis mox evanescentibus, inferne e 6-10 seriebus cellularum formatis ; costa homostrôsica, heterodictya $\left({ }^{\star}\right)$, leucocystis ubique bistratosis, breviter rectangulis vel subhexa-

" J'appelle nervure hétérodictyée celle qui, bien que pouvant être homostrôsique, présente cependant une structure basilaire distincte, par opposition à la nervure homodictyée, possédant la même structure dans toute sa longueur. „ 
gonis, poris paucis, parum distinctis vel nullis, chlorocystis inferne hypercentralibus, in costæ majore parte centralibus, apicem versus distincte hypocentralibus, stereomate mediano dorsali, apice pro more dilatato et denticulato vel tuberculoso, stereomatibus marginalibus altero ambabusve superne minute et remote serrulatis. Cætera desunt.

Comores: Mayotte, mont Sapéré (Marie ; Hb. Bescherelle, sub. nom. L. Hildebrandtii C. Müll.).

"Cette espèce se distingue du L.Hildebrandtii C. Müll. de la même région, avec lequel M. Bescherelle l'a confondue, par sa teinte moins pâle, un peu verte ou jaunâtre, par ses stéréomes marginaux finement mais distinctement denticulés dans la partie supérieure, au moins d'un côté, et par les leucocystes de la nervure plus petites, les supérieures plus courtes. En outre, le stéréome médian du L.Hildebrandtii est, en général, plus fortement denté au sommet que celui du $L$. mayottense.

Le $L$. angustifolium Ren. et Card. de la Réunion est aussi très voisin du L. mayottense; il en diffère cependant par ses tiges très courtes, presque nulles, ses feuilles encore plus allongées, et ses leucocystes supérieures encore plus courtes, presque carrées.

L. Seychellarum Besch. se distingue de l'espèce nouvelle par ses leucocystes plus courtes, ses pores très petits, mais bien distincts, et par seschlorocystes plus petites, hypocentriques dès le milieu de la feuille. D

L. Renauldii Card. in Bull. Hb. Boissier. - Tenellum, fragile, albescens, gregarium vel laxe cespitosum, subacaule, vix o, 5 cent. altum. Folia erecto-patentia, anguste lineari-lanceolata, longe acuminata, 3-4,25 mil. Ionga, 0,25-o,3 lata, plicato-canaliculata, apicem versus deplanata, obtusa subacutave, alis latiusculis, pro more inæqualibus, medium versus evanescentibus, inferne e 4-6 seriebus cellularum formatis; costa homostrôsica, heterodictya, leucocystis ubique bistratosis, inferioribus breviter rectangulis, cæteris subquadratis vel hexagonis, chlorocystis in costæ majore parte pro more distincte hypercentralibus, apicem versus hypocentralibus, stereomate mediano dorsali, interdum in pagina ventrali quoque emergente, apice dilatato dentibus numerosis vel papillis dentiformibus horrido, stereomatibus marginalibus integris vel apicem versus minute et remote serrulatis. Cætera desunt.

Madagascar : forêt d'Alakaty (Perrot frères); cercle de Fort-Dauphin : district de Mahinpoana (Hb. G. Paris).

a Diffère du L.Hildebrandtii C.Müll., des Comores, avec lequel on l'a confondu, par sa petite taille, ses feuilles plus petites, à denticulation apicale moins forte, ses leucocystes supérieures plus courtes, carrées, brièvement rectangulaires ou hexagonales, et ses chlorocystes en général distinctement hypercentriques jusque près du sommet. C'est la plus petite des espèces du domaine malgache. „ 
L. Hildebrandti C. Müll.

Les localités malgaches d'Alakaty et Vohémar (Perrot) citées dans le Prodrome, doivent être supprimées et cette dernière attribuée au L. Rodriguez̧ii C. M.

L. Rodriguezil C. Müll.

Madagascar: Vohémar Perrot; forêt de Fito dans le district de Tamatave (Perrot in Hb. Levier) ; Beforona entre la forêt d'Analamazoatra et Andevorante Dorr, 1906; sine loco, com ${ }^{\text {it }}$ Friren, (Hb. Cardot).

\section{Tribu III. - OCTOBLEPHAREE Card.}

Nervure sans faisceau scléreux. Chlorocystes dans une seule assise dans toute la longueur de la nervure, celles de la partie supérieure toujours de section trigone et placées au point de jonction de trois leucocystes; celles de la partie inférieure de section souvent quadrangulaire.

\section{Gen. Cardotia Besch.}

Nervure déprimée, concave, canaliculée dans le haut, sans faisceau scléreux, hétérostrosique ou subhétérostrosique; 2 couches de leucocystes dans la partie moyenne et supérieure, 3 à 5 dans le bas. Ailes étroites, pas de margo. Tige dépourvue de faisceau axile distinct. Fructification inconnue.

C. Heterodictya Besch. (Leucobryum heterodictyon Besch.)

Madagascar : île Sainte-Marie (Marie, Perrot, Hb. Levier): province de Fianarantsoa (Hb. Paris, forme passant à la var. Boiviniana).

Var. Boiviniana Card. (Leucobryum Boivinianum Besch.).

Madagascar : île Sainte-Marie (Boivin, Marie, Rev. Arbogast, Perrot).

"Cette var. diffère du type par ses dimensions plus réduites, ses feuilles dressées, imbriquées, plus petites et plus étroites (long 4 à 5 mill., larg. 0,75 à 0,8 mil.) et par sa nervure plus mince subhétérostrosique. Certains échantillons établissent la transition entre les 2 formes. 


\section{Gen. 0ctoblepharum Hedw.}

Nervure épaisse et large, arrondie sur le dos, de section semicirculaire dans le bas, ordinairement plus ou moins déprimée, de section elliptique, ovale ou subtrigone dans la partie moyenne, plus déprimée vers le sommet, sans faisceau scléreux; leucocystes en couches nombreuses ( 3 à 7 dans le bas, 6 à I I vers le milieu, 2 à 4 au sommet). Ailes assez larges dans la partie inférieure, ordinairement très inégales, courtes et disparaissant complètement à peu de distance de la base, pas de margo. Tige sans faisceau axile et sans cellules corticales différenciées. Fructification latérale ou terminale. Capsule symétrique, dressée, longuement pédicellée. Coiffe dimidiée. Péristome non dicranoïde.

O. Africanum (Broth.) Card. (Arthrocormus africanus Broth.).

Madagascar : île Sainte-Marie, Marie.

"Cette espèce diffère de toutes les autres espèces de ce genre connues jusqu'à ce jour par ses chlorocystes qui restent carrées jusqu'au-delà du milieu de la nervure et ne deviennent trigones que dans la partie supérieure. La nervure est de section semicirculaire. Les feuilles mesurent de 4 à 5 mil. de long. sur o, 6 à 0,75 de large dans la partie basilaire. Le péristome est formé de 16 dents blanchâtres, lisses, lancéolées-subulées, réunies 2 à 2 par une membrane hyaline très délicate. "

Gen. Fissidens Hedw.

A. - Folia immarginata.

F. Zippelianus Doz. Molk.

Madagascar: Cercle de Maintirano, Ranobé, 19or. (Hb. Paris).

Subspec. ${ }^{*}$ F. Savellii Par. Ren. (F. Savellii P. R. in Rev. bryol. Igo2, p. 2). - Cæspites laxi superne saturate virides, inferne fuscovariegati. Caulis circa $\mathrm{o}^{\mathrm{m}}$ o $\mathrm{I}$ altus, usque basin versus foliosus. Folia 15-20 juga, inferiora remotiuscula, superiora magis conferta, humida subsecunda, sicca plus minus crispatula $23 / 4-3$ mil. longa, lineali-lanceolata, immarginata. Lamina vera ad $2 / 3$ vel $3 / 4$ producta; lamina dorsalis apice brevi acuminata, interdum acutiuscula vel rotundato- 
obtusiuscula, basilari haud decurente rotundata vel truncata, auriculam efformante, marginibus integris, costa basi $47 \mu$ lata sub apice finiente, rete viridi subobscuro, cellulis basilaribus secus costam in lamina vera paulo majoribus hexagono-rectangulis, cæteris omnibus subaqualibus subrotundis 3-5 $\mu$ latis, marginalibus paulo majoribus apice oblique prominentibus. Cætera ignota.

Madagascar: province de Majunga, Manpikony, Igor, SAvelli (Hb. Paris).

Cette sous-espèce très voisine du $F$. Zippelianus $\mathrm{Dz}$, et Mk. de Java, s'en distingue par la base de la lame dorsale qui a la forme d'une oreillette arrondie ou tronquée séparée de la nervure par un sinus étroit très distinct. Dans les figures du Bryologia javanica et dans un échantillon du $F$. Zippelianus communiquépar M. Fleischer, la base de la lame dorsale naît de la nervure par une décurrence gonflée ou non audessus du point d'insertion, mais sans la moindre trace de sinus.

F. ovatus Brid.

Madagascar: Ivondro (Hb. Paris). Plante fertile, fruit terminal, pédicelle pâle, long de 7 mil., courbé au sommet, capsule pendante, petite (long $3 / 4$ mil.). Opercule très longuement rostré.

Var. ochroloma Ren. Card.

Diffère du type de La Réunion par ses feuilles un peu plus courtes, plus étroites, décolorées d'un côté sur les marges par une assez large bande hyaline complètement dépourvue de chlorophylle, mais sans changement dans la forme des cellules.

Madagascar: Betsileo, Rev. Villaume, I904; Ambohibeloma, Rev. Camboué (Hb. Lacouture).

Le $F$.ovatus Brid. tel qu'il faut le comprendre d'après l'échantillon de La Réunion rapporté par Bescherelle à cette espèce, diffère du $F$. Boivini Besch. par sa fronde très large, par sa lame verticale tronquée ou arrondie à la base, et du $F$. Zippelianus D. M. par ses feuilles du double plus grandes, par les cellules marginales du sommet non ou très peu différenciées. Le $F$. Boivini Besch. a les feuilles toujours obtuses et la lame verticale naissant au-dessus de la base par une décurrence étroite.

F. Borvini Besch.

Madagascar : Fianarantoa, Villaume, rgo5; Ambohibeloma, CanBoute, I 906 . (Hb. Lacouture).

F. Maniæ Par. Ren. - Ex habitu F. Boivini persimilis. Cæspites sat densi, viridi-lutescentes. Caulis erectus $0,02-0,025$ longus, superne 
2-3 furcatus. Folia 30-40 juga etiam húmida valde incurvata, sicca crispata, I I /2-2 mil. longa, e basi oblongo-lanceolata subaciniformia, elimbata, integerrima. Lamina vera ultra medium producta, lamina dorsalis costæ basin versus enascens abrupte vel rotundato-truncata, haud attenuata, apicalis inflexa obtuse acuminata vel obtusa, rarius acuta. Costa cum vel sub apice evanida. Cellulis obscuris hexagono-rotundatis, papillosis papillis, dorso rotundate prominentibus, marginalibus haud dentiformibus. Cætera desunt.

Madagascar : province d'Antsirabe, Galinon, 1903 (Hb. Paris).

Cette espèce se distingue du $F$. Boivini Besch., dont elle a le port, par la forme variable de l'acumen, tantôt aigu, tantôt plus ou moins obtus, par la lame dorsale non atténuée à la base; tandis que dans l'espèce voisine, cette lame est toujours très étroite, décurrente ou rarement tronquée assez haut au-dessus de la base.

Par la forme de ses feuilles, le $F$. Mania se rapproche du $F$. Zippelianus D. M., mais ce dernier a un autre port, plus trapu, des feuilles plus grandes étalées, pectinées à l'état humide; en outre les cellules marginales sont plus grandes, plus régulières, carrées ou subrhombées et forment une série très distincte des cellules internes.

\section{B. - Folia plus minus marginata.}

F. LURidus Ren. Card.

Madagascar: province de Betafo, Janjanina, I90I (Hb. Paris).

F. obsoletidens Besch.

Madagascar : Cercle de Maintirano (Hb. Paris) à Tsimandrebabaka, 1902.

Feuilles raides pectinées. Tissu transparent composé de cellules assez grandes, le tissu du I/3 inférieur lâche, composé de cellules allongées rectangulaires subhexagones à parois minces. Lame dorsale étroite décurrente au-dessus de la base. N'avait pas encore été signalé en dehors de Nossi-bé et Nossi-Comba.

F. Motelayi Ren. Card.

Madagascar: Cercle de Maintirano, Ranobé(Hb. Paris), Anamoissy, 1902.

\section{Gen. Hyophila Brid.}

H. Potieri Besch. var, atroviridis Ren. Card. - A planta typica differt colore atro viridi, statura multo robustiore (caulis 15 mil. altus) 
foliis siccis laxe contortis, latioribus, ellipticis, apice plerumque subrotundatis, marginibus planis vix involutis. Forsan species propria?

Madagascar: Ambohibeloma, Cumbové, 1907 (Hb. Paris).

H. DORrII Ren. Card.

Madagascar : Cercle de Maintirano, Dembavy (Hb. Paris).

H. clavicostata Ren. Card.

Madagascar: Cercle de Maintirano, 1901, (Hb. Paris).

H. Girodi Ren. Card. (Pl. x). - Dioica. Cespites virides, densi, tapetis ad instar longe lateque extensi. Caulis circa 8 mil. altus, strictus, basi radiculosus, tota fere longitudine foliosus, plus minus divisus. Folia humida stricta, sicca crispula, e basi paulo dilatata sensim angustata, anguste lanceolata, apice subobtusa, 2,5 mil. longa, marginibus planis,

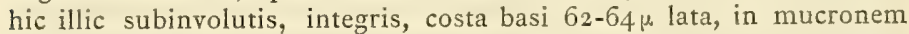
brevissimum pro more bidentatum vix excedente, canaliculata, dorso tota fere longitudine grosse tuberculosa, rete superiore obscuro, cellulis chlorophylosis minutis hexagono-quadratis $6 \mu$ latis, dense et minute papillosis, rete basilari achlorophylloso pellucido usque ad tertiam partem folii producto, e cellulis quadratis, infimis breviter rectangulis efformato. Capsula in pedicello 5-7 mil. longo erecta, cylindrica, I, 5 mil. longa, gymnostoma.

Madagascar : île Sainte-Marie, sur le sable de la plage, Girod Genet, I 899 .

Cette espèce, que l'on peut rapprocher de l'H. usambarica Broth. de l'Afrique orientale, en differe par sa taille moindre, par ses feuilles presque de moitié plus petites, par la base hyaline occupant tout le tiers inférieur de la feuille, par l'acumen subobtus à peine dépassé par l'excurrence de la nervure, celle-ci munie sur le dos, presque jusqu'à la base, de grosses papilles tuberculeuses, tandis qu'elle est lisse dans l'espèce voisine.

H. Sakalavensis Par. Ren. (Pl. xI). - Cæspites incohærentes laxiusculi saturate virides. Caulis erectus gracilis $4^{-5}$ mil. altus. Folia subremota sicca incurvato-subcrispula I 3/4-2 mil. longa, $1 / 2$ mil. lata, e basi oblonga sensim angustata, lanceolata summo subrotundata, interdum late et obtusissime acuminata, haud mucronata, marginibus parce involutis vel planis, integris rarius apice obsolete erosulis haud crenulatis. Costa valida $36-38 \mu$ lata sub apice evanida, dorso subtiliter 
granulosa vix papillosa. Cellulis basilaribus rectangulis inanibus, mediis quadratis, superioribus $6 \mu$ latis subhexagonis vel rotundatis, dense papillosis, obscuris. Cætera desunt.

Madagascar: Cercle de Maintirano, Igor (Hb. Paris).

Differe du $H$. Potierii Besch. par les feuilles plus allongées et plus étroites, oblongues-lancéolées, longuement rétrécies dans la moitié supérieure, arrondies au sommet, non mucronées, à marges entières non crénelées. Se distingue du H. lanceolata R. C. par la forme du sommet de la feuille et les marges entières, du $H$. angustifolia Par, et Ren. par les feuilles moins étroites à tissu plus large, non cucullées ni mucronées.

H. leioneura Ren. Par. (Pl. x). - Cæspites saturate virides basi terra obruti. Caulis rigidus, erectus, simplex, 6-1o mil. altus, basi fere denudatus. Folia inferiora remota superiora densius conferta in gemmam clavatam contorta 1 I $/ 2$ mil. longa, e basi dilatata 0,5 mil. lata fere sensim angustata acuta vel subacuta, marginibus integris, planis. Costa crassa in mucronem brevem acutum pro more $1-2$ dentatum excedente, dorsolævi vel sublævi. Rete basilari densiusculo e cellulis inanibus breviter rectangulis efformato, cellulis superioribus hexagonis $6 \mu$ latis dense papillosis, obscuris. Cætera desunt.

Madagascar : province de Moramanga, Lovver. (Hb. Paris).

Cette espèce se distingue immédiatement par ses tiges raides, un peu espacées, non contiguës, encombrées de terre à la base. Par la forme des feuilles elle se rapproche du H. Girodi R. C. de Sainte-Marie de Madagascar; mais cette dernière a un autre port, s'étale en tapis denses, et ses feuilles sont plus longues, dilatées à la gaîne, puis plus rapidement rétrécies, et la nervure est couverte dans presque toute sa longueur de grosses papilles tuberculeuses, tandis qu'elle est à peu près lisse dans l'espèce voisine.

Le H. usambarica Broth. de l'Usambara se rapproche aussi de notre plante; mais la taille est plus robuste, les tiges sont densément feuillées dès la base, les feuilles sont plus grandes (2 $\mathrm{r} / 2-4 \mathrm{mil}$.) et la nervure est excurrente en un mucron beaucoup plus long.

II. angustifolia Par. Ren. (Pl. xI). - Cæspites sat densi, luteovirides. Caulis erectus circa 5 mil. altus radiculosus. Folia dense conferta sicca crispula, I 3/4-2 I/4 mil. longa, o,3 mil.lata, lineali-lanceolata, marginibus fere parallelis planis, medio undulatis, integris, summo in acumen obtusum pro more cucullatum subito involutaceo-conniventibus. Costa valida $36 \mu$ lata percurrente vel in acumen brevissimum excedente, dorso lævi. Cellulis basilaribus inanibus ad costam rectangulis vel 
rhombeo-rectangulis, marginalibus multo angustioribus, superioribus minutis papillosis obscuris. Cætera desunt.

Madagascar: province de Betafo, rive droite de la Mania, Galinon, rgor. (Hb. Paris).

Cette plante paraît être très voisine du $\boldsymbol{H}$. Holstii Broth. de l'Usambara. D'après la description de l'auteur (Engler's Bot. Jahrb. 1894, p. 183) cette dernière semble n'en différer que pas les feuilles plus longues 3 1/2.4 mil. ainsi que par l'acumen aigu non cucullé.

\section{Gen. Pottia Ehrh.}

P. (?) punctulata Ren. Par. (Pl. XII). - Cæespites laxiusculi rufescentes. Caulis subsimplex circa 7 mil. altus apice obtusus. Folia humida erecta, sicca incurvata, passim vage subspiraliter torta I I/2 mil. longa, ovato-acuminata, apice obtusa, marginibus integris basi planis mox usque ad summum revolutis. Costa pervalida superne paulo dilatata vix papillosa cum apice finiente vel vix clavato - excedente, cellulis basilaribus ad costam majoribus, lævibus, hyalinis, rectangulis, ad marginem numerosis $12-15$ seriatis quadratis, passim vage subhexagonis papillis $2-6$ majusculis ornatis, dorso et marginibus dense et subtiliter prominentibus. Cæatera desunt.

Madagascar: province de Betafo, Janjanina (Hb. Paris).

Diffère du Pottia (Anacalypta) apiculata (Kiaër) C. M. par un port plus robuste, les feuilles largement ovalès à la base puis rapidement rétrécies, non crispulées à l'état sec, par la nervure plus forte un peu renflée au sommet qu'elle atteint ou qu'elle dépasse sans former d'apicule, à papilles peu saillantes, à cellules carrées plus grosses munies de papilles arrondies très distinctes.

P. (?) tuberculosa Ren. Par. (Pl. xil). - Cæspites humiles laxiusculi saturate virides terra obruti. Caulis erectus 5 mil. altus debilis flexuosus inferne radiculis paucis ornatus. Folia inferiora remota patulorecurva, superiora confertiora erecto-patentia sicca apice subcrispata, oblonga, 0,5-0,7 mil. longa sublingulata apice rotundata, sæpius brevi et late acuminata interdum apiculata, marginibus integris sed ob cellulas marginales papillosas apice prominentes valde asperis; costa tenui dorso papillosa, basi $17 \mu$ lata in apiculum brevem acutum pro more exeunte, rarius sub apice evanida. Rete dimidii inferioris pellucido e cellulis longioribus hexagono-rectangulis magnis fere inanibus epapil- 
losis efformato, cellulis mediis et superioribus exacte hexagonis pro folii magnitudine amplis, usque г granulosis et papillosis, papillis dorso prominentibus. Cætera desunt.

Madagascar: Cercle de Maintirano, 190 I (Hb. Paris).

En l'absence de fructification, il est difficile d'assigner à cette espèce unie place générique certaine. Par le port et l'ensemble des caractères de végétation, elle a de l'analogie avec les petites espèces de Splachnobryum qui croissent dans l'ile voisine de Nossi-Bé; toutefois le tissu est plutôt celui d'un Pottia de la section Splachnobryella C. M. avec les cellules de la moitié supérieure de la plante amples, régulièrement hexagones, remplies de grains chlorophylleux distincts et d'une grosse papille nettement saillante sur la face dorsale, les- marginales festonnées à leur paroi extérieure. On peut la comparer au P. vernicosa $\mathrm{Dz}$. et Mk. de Java dont elle se distingue d'ailleurs facilement par les feuilles non spatulées, par la nervure plus longue généralement excurrente en un court apicule aigu, par les papilles plus fortes, très grosses. Le P. apiculata (Kiaër) C. M. (Barbula Kiaërii Broth.) est plus robuste et son tissu foliaire est plus dense, composé de cellules beaucoup plus petites, finement et densément papilleuses; la nervure est couverte dans presque toute sa longueur de grosses papilles arrondies.

Le Barbula (?) madagassa R. C. a les feuilles beaucoup plus grandes, rétrécies obtusément acuminées, nettement dentées au sommet avec des cellules moyennes carrées dont le diamètre n'excède pas $6 \mu(*)$ : la nervure forte $(25-28 \mu)$ est distinctement évanouissante.

\section{Gen. Trichostomum Hedw.}

T. Pennequini Ren.Par.-T. mutabili B.E. europ. peraffine differt : caulibus multo minoribus $6.8 \mathrm{mil}$. altis sparsim inter muscos vigentibus, foliis siccitate magis crispatis, rete basilari hyalino, delicatulo, in tota latitudine æquali, e cellulis elongate-hexagonis tenuiter limitatis composito, nec non pedicello breviore. Capsula operculata immatura in pedicello pallido tantum 6 mil. alto, erecta, o, I 5 mil. longa, basi attenuata, oblonga. Operculun conicum recte et sat longe rostratum e cellulis recte seriatis reticulatum. Peristomium, ut videtur, rudimentarium.

Madagascar : Cercle des Baras, Ivondro; Lespagnol et Croll (Hb. Paris).

* La place générique de cette plante est encore indécise. M. Brotherus, dans les Pflanżen Familien, croit plutôt à un Hyophila, ce qui est possible. Cependant mon ami M. Cardot et moi avons hésité à la rattacher à ce genre à cause du tissu de la lame chlorophylleuse moins obscur et moins dense que dans les Hyophila. Cette plante appelle de nouvelles observations. 
T. (Hydrogonium) Brotheri Ren. Par. (Pl. xiv). - Diö̈cum. Cæspites laxiusculi pallide vel luteo virides inferne terra calcarea obruti. Caulis rigidus $2-3$ cent. longus, fusco-purpureus, erectus, rigidus, subsimplex cradiculosus. Folia in dimidio inferiore vel fere ad apicem remotiuscula indumento tophaceo vestita, erecto-patentia, superiora sicca crispatula e basi oblonga decurrente late lanceolato-ligulata, apice latissime acuminato-rotundata, interdum subtruncata, 2 mil. longa, basi $0,4-0,5 \mathrm{mil}$. lata. Costa basi $30 \mu$ lata, valida, sub apice haud cucullato distincte evanida, dorso lævi; marginibus planis passim breviter revolutis, integerrimis. Rete laxiusculo tota longitudine usque ad apicem pellucido, cellulis lævibus inanibus, basilaribus laxis ad costam latioribus elongate subhexagonis marginalibus quadratis. Flores feminei in coma absconditi, autheridii 8-1o eparaphysatis. Cætera inquirenda.

Madagascar: ad moles tophaceas, Cercle de Maintirano, Igo I (Hb. Paris).

Cette belle plante zrès voisine du $T$. Ehrenbergii -Ltz., n'en diflère guère que par son tissu hyalin dans toute la longueur de la feuille jusqu'au sommet et ses cellules supérieures vides et lisses ainsi que le dos de la nervure.

Dans le T. Ehrenbergii Ltz., (T. mediterraneum C. M.) var. Algerice C. M. du Rummel (Constantine), les touffes sont plus molles, la tige grêle non rigide, les feuilles un peu pluslongues, le tissu plus dense, les cellules basilaires plus étroites, rectangulaires, la nervure un peu plus large $(35 \mu$ à la base), un peu rugueuse sur le dos au sommet, les cellules de la moitié supérieure remplies de matière verte et munies de papilles espacées, larges, arrondies et très distinctement saillantes sur le dos de la feuille.

On attribue, il est vrai, au type de Marseille un tissu un peu plus lâche que dans la var. Algerice; mais l'acumen est souvent subaigu et cucullé. Les cellules de la moitié supérieure sont fortement chlorophylleuses et légèrement papilleuses, la nervure est un peu plus forte.

D'après C. M., le type du T. Ehrenbergii Ltz. du Sinaï, differerait du T. mediterraneum C. M. par un port plus robuste, des feuilles plus larges, obtuses et cucullées au sommet et le tissu plus lâche dans toute la longueur de la feuille. Toutes ces différences sont légères, et bien que l'absence de chlorophylle et de papilles dans la moitié supérieure de la feuille du $T$. Brotheri, laquelle-reste hyaline jusqu'au sommet, ne constitue pas un caractère bien important, il permet tout au moins de considérer cette plante de Madagascar comme une sous-espèce ou race régionale du T. Ehrenbergii Ltz.

\section{Gen. Leptodontium Hpe.}

L. epunctatum C. Müll.

Comores: Grande Comore ( $\mathrm{Hb}$. Paris). 
Le type de la Réunion dont la tige est haute de 3-4 cent. est intermédiaire entre la var. paludosum Ren. Card et le L. Hildebrandtii C. Müll. Dans la var. paludosum la tige est longue de $6-8$ cent., les feuilles sont longues de 3 mil., très aiguës, dentées dès le $1 / 3$ inférieur et fortement au sommet. Dans le $L$. Hildebrandtii C. Müll., les feuilles sont plus courtes, 2 mil., largement acuminées, moins fortement dentées et seulement au sommet. Cette forme paraît être celle des lieux secs et il est difficile de la considérer comme plus qu'une variété de L. epunctatum C. M. La var. paludodosum R. C. a été retrouvée en 1894 au Mont Antaby près d'Ambositra par le Docteur Forsyth Major (Hb. Levier) et à Ambositra par le Rev. Villaume en Igo4 (Hb. Lacouture), c'est-à-dire dans la même région où elle avait été découverte en 1892 par le Rev. Berthieu.

Gen. Didymodon Hedw:

D. subrevolutus (Hpe) Broth. in Pflan «en Familien $=$ Barbula subrevoluta Hpe.

Gen. Barbula Hedw.

B. mucronulata Ren. Card. (Pl. xII). - Lutescens, laxe cæspitosa. Caulis erectus, strictus, simplex, 5-8 mil. longus. Folia madida erectopatentia, sicca erecto incurva, subcanaliculata, I-I,25 mil. longa, oblonga, superne lineali-lingulata, apice obtuso minute mucronulata, marginibus planis vel basi subrevolutis integerrimis ; costa valida lutescente, dorso minute papillosa, in apiculo pellucido producta ; rete densissimo, crasso obscuro, cellulis parvis rotundo-quadratis, minutissime sed dense papillosis, basilaribus laxioribus, lævibus, pellucidis, breviter rectangulis, juxtamarginalibus quadratis. Cætera ignota.

Madagascar : entre Tananarive et Betafo, Rev. Caussėque.

Espèce omise dans le Prodrome, que l'ọ pourrait comparer au B. commutata Jur. d'Europe, mais qui s'en distingue par ses feuilles non ondulées, planes aux bords et obtuses-mucronées. Elle a été retrouvée fertile dans la province d'Antsirabe ( $\mathrm{Hb}$. Paris), ce qui permet de compléter la description. Dioïca. Capsula in pedicello 10-1 2 mil. longo basi rubello, superne pallido, sinistrorsum torto, exacte cylindrica, 2 mil. longa, etiam vetusta pallida, nitida ore purpureo. Peristomii dentes purpurei, papillosi e membrana basilari brevissima nascentes, $\mathrm{r}$ mil. longi, bis laxe torquati. Operculum longe subulatum purpureum. Sporæ læves minimæ 6-8 $\mu$ crassæ.

B. sporaphylla Ren. Card. - Inter muscos (Brachythecium Borgeni $\mathrm{Hpe}$ ) sparse crescens. Caulis simplex vel superne parce divisus, 
pergracilis subfiliformis, 5-10 mil. altus, erectus, flexuosus. Folia remota humida erecto-patentia, sicca tortilia, I $1 / 4-11 / 2 \mathrm{mil}$. longa $1 / 5 \mathrm{mil}$. lata, linealia, marginibus parallelis, apice obtusata, mucronata; marginibus planis papilloso-hirsutis; costa basi $30 \mu$ lata, canaliculata apice abrupte evanida, dorso albido-nitida. Rete obscuro, cellulis superioribus obscuris, papillis obrutis, hexagonis, mediis quadratis, basilaribus hyalinis $1 / 6$ folii occupantibus, elongate-rectangulis, marginalibus angustioribus, cellula apicali hyalina, apiculum acutum efformante, basi plerumque dentibus $1-2$ prædita.

Madagascar : Ambohibeloma, Rev. CAmbouḱ, 1906-1907. (Hb. Lacouture).

On ne peut guère comparer cette espèce qu'au Barbula sparsifolia Ren. Card. de La Réunion. Elle s'en distingue à première vue par un port beaucoup plus grêle, la tige filiforme et les feuilles encore plus espacẻes, de moitié plus petites et encore plus étroites. En outre le tissu est plus obscur, la nervure papilleuse sur le dos et les marges sont formées par une rangée de cellules transversalement dilatées et crénelées fortement par la saillie des papilles. L'apicule est formé par une grande cellule unique, conique,portant quelquefois $\mathbf{I}-2$ dents à la base,et n'est pas le prolongement de la nervure qui cesse brusquement sous le sommet, tandis que dans l'espèce voisine, le mucron est formé distinctement par le prolongement de la nervure et se compose de plusieurs cellules rectangulaires jaunâtres.

B. (Syntrichia) subrufa Ren. Card. (Pl. xI). - Cæspites extensi, molles, intus rufescentes vel fuscescentes, superne olivaceo-virides. Caulis subsimplex 3,5-4 cent. altus in medio inferiore valde radicolosus. Folia humida patula subquarrosa, sicca incuryata, carinata subspiraliter torta $3 \mathrm{mil}$. longa, I I $/ 4 \mathrm{mil}$. lata, lingulata apice rotundata, haud emarginata, marginibus papillis prominentibus crenulatis, in medio inferiore paululum revolutis, vel sæpe uno latere planis. Costa rubra dorso paulo papillosa, in apiculum obliquum e cellula unica formatum, excurrente. Cellulis chlorophyllosis parvis quadratis, papillosis, obscuris basin versus secus margines lamina $5-6$ seriata (cellulis paulo longioribus) descendentibus. Cellulis basilaribus juxtacostalibus magnis, hyalinis, rectangularibus vel subrectangularibus $\mathrm{I} / 3$ folii longitudinis occupantibus.

$$
\text { Madagascar: Fianarantsoa, Rev. Villaume, igo4 (Hb. Lacouture). }
$$

Cette espèce paralt voisine de $B$. intermedia var. calva d'Europe et n'en diffère que par des caractères légers : taille plus élevée, feuilles plus grandes arrondies au sommet, très brièvement mucronées; cellules basilaires marginales plus courtes et plus larges, la plupart carrées. 
Dans la var. calva d'Europe les feuilles sont moins nettement arrondies' au sommet et le limbe accompagne généralement un peu le mucron qui est plus épais et plus long, composé en largeur de 3-4 séries de cellules et, dans quelques feuilles,prolongé en poil rudimentaire court, hyalin; les cellules basilaires marginales sont rectangulaires $2-3$ fois plus longues que larges. Dans le $B$. subrufa R. C. le mucron fait brusquement saillie sur le sommet arrondi de la feuille et reste uniformément triangulaire, unicellulaire, sans jamais s'allonger ni se prolonger en poil rudimentaire.

Ces différences sont légères, mais constantes et les fruits, quand ils seront connus pourront fournir d'autres caractères.

Le B. (Syntrichia) rufa Sch. de Maurice a les feuilles plus longues, plus étroites, terminées par un poil assez long denticulé.

\section{Gen. Streptopogon Wills.}

Dans sa monographie du genre Streptopogon (Annals of Botany, vol. xvIr, no ${ }^{2} \times v$, January 1903), M. E. S. Salmon a réduit le nombre des espèces citées jusqu'alors. Par suite de ces modifications, les espèces de Madagascar sont rattachées aux 2 types suivants :

S. erythrodontus (Tayl.) Wills ex Mitten in Hook. Journ. of Bot. (1851).

Var. Rutenbergii (C. Müll.). "A planta typica austro-americana differt foliis plerumque distantioribus et patulis vel patentibus, limbo angusto ad folii summum apicem producto vel longe infra apicem desinente, cellulis laxioribus ».

Madagascar: forêt d'Ambatondrazaka, Rutenberg, sub S. Rutenbergii C. M. ; Imerina, Andrangoloaka, Hildebrandt, sub S. Hildebrandtii C. M.; Central Madagascar, PARKER, sub S. Parkeri Mitt.

S. rigidus Mitt. in Spruce Cat. Musc. Amazon. et And. (1867).

Madagascar : Ambatondrazaka, Rutenberg, sub, $S$. calymperes C. Müll.

Le S. (?) Mayottensis Besch. est, d'après Brotherus, une Funariacée.

Gen. Galymperes Sw.

C. (Hyophilina) Mathieui Ren. Card. (Pl. xv). - Humile, saturate viride. Caulis perbrevis, 2-5 mil. altus. Folia sicca laxe crispula, 3 mil. 
longa, e basi vaginante fere dimidiam partem folii occupante, spathulata, apice rotundata, marginibus omnino planis, in tertia parte superiore, ob cellulas marginales apice acute prominentes, subtiliter denticulatis, papillis dorsalibus densis paulo prominulis, costa basi $42 \mu$ lata, in mucronem brevissimum excedente, inferne laevi, in lamina chlorophyllosa dorso rugulosa, cancellinis ovatis vaginam superantibus, e cellulis 12 seriatis rectangulis, paulisper longioribus quam latioribus, nonnullis rhombeis intermixtis, efformatis, teniolis e cellulis $3-4$ seriatis compositis, usque ad $2 / 3$ laminæ viridis productis, cellulis juxtamarginalibus ad ventrem 3-4 seriatis exterioribus apice leniter dentiformibus. Fructus ignotus.

Madagascar : Maroantsetra, baie d'Antongil, MATHIEU,(Hb. Cardot).

Espèce du groupe Climacina, différant du C. Nossi-Combe Besch. par les feuilles spatulées, arrondies au sommet, très brièvement mucronées par l'excurrence de la nervure, et par la base vaginante plus développée, atteignant presque le milieu de la feuille.

C. (Hyophilina) erosulum Ren. Par. (Pl. xv). - Caulis brevis 3-4 mil. altus, apice 2-3 furcatus. Folia sicca incurvato-cirrata $2-2 \mathrm{I} / 2$ mil. longa plerumque anomala, normalia e basi vaginante obovata ad I/3 folii producta lanceolata, late et obtuse acuminata, marginibus planis superne eroso-serratis. Costa circa $47 \mu$ lata sub summo apice evanida, plerumque longe proboscidea, dorso nisi infima basi grosse tuberculosa. Cellulis chlorophyllosis quadratis, dorso densissime acute subtiliter papillosis, papillis vix prominulis. Cancellina obovata vaginam superans e cellulis breviter rectangulis 8-1o seriatis composita. Tæniola 3-4 cellulata usque apicem fere producta supra ventrem sæpe geminatim dentata Syrrhopodontis ad instar. Cellulis marginalibus ad ventrem quadratis $4-5$ seriatis, externis breviter dentiformibus, basilaribus, hyalinis rectangulis 2 seriatis.

Madagascar: Cercle de Maintirano, Beravina, VIDAL ,(Hb. Paris).

Cette espèce du groupe Climacina, qui paraît voisine du C. loucoubense Besch., en diffère, d'après la description de son auteur, par les feuilles obtusément acuminées, la téniole prolongée dans la lame verte, par la nervure tuberculeuse dès le milieu de la base vaginante, enfin par les cellules marginales bisériées à la base et $4^{-5}$ sériées vers le ventre de la gaîne; celle-ci est aussi plus allongée.

Le C. Mathiai R.C. de la côte Est de Madagascar a aussi quelques rapports avec notre plante ; mais il en diffère essentiellement par les feuilles plús grandes spatulées, brièvement mucronées, à peine denticulées, par la nervure lisse dans la moitié inférieure, légèrement rugueuse vers le sommet seulement. 
C. decolorans C. Müll. var. latifolium Ren. Par. - A planta typica differt colore luteo vel citrinello variegato, foliis multo latioribus (1 $\mathrm{i} / 4$ mil.) apice rotundatis vel subtruncatis, costa latiore $(80-85 \mu$ loco $60-65 \mu$ ), teniola paulo angustiore et cellulis marginalibus partis vaginantis magis dentiformi prominentibus. Forsan species propria.

Madagascar: province d'Ankazobé, Fihaonana, (Hb. Paris).

Comparée au type de Mayotte, cette forme est plus robuste, le port est plus rigide, les feuilles de $\mathrm{r} / 3$ plus longues et plus larges, ce qui fait paraître plus étroite Ia téniole qui ne comprend à la base que $2-3$ séries de cellulès et 3 sur le milier de la gaîne, tandis qu'on en compte 3.4 dans le $C$. decolorans type. C'est peut-être une espèce propre, toutefois les échantillons sont trop maigres pour qu'on puisse être bien fixé à ce sujet.

\section{Gen. Syrrhopodon Schwgr.}

S. Chenagoni Ren. Card.

Madagascar: province d'Ambositra, Ivanana, Frissard, 1905, (Hb. Paris).

S. hispido costatus Ren. Card.

Madagascar : Tamatave, Perrot, i 897 ; province d'Anosibe, vallée de l'Ofika, Verdolin, igor, (Hb. Paris).

S. graminifolius Ren. Card.

Madagascar: Tamatave, Perrot, 1897.

S. glaucophyllus Ren. Card.

Madagascar : province d'Andevorante, Antseranandratsy, 1900, (Hb. Paris).

(Folia perichetialia caulinis similia, limbo hyalino minus distincto, interdum interrupto. Capsula cylindrica castanea, I $1 / 4$ mil. longa in pedicello I I mil. alto rubello; lævi erecta. Vaginula cylindrica archegoniis vestita. Peristomii simplicis dentes brevès, obtusi, pallide lutei, granulosi, 3-4 articulati. Sporæ aureæ, parvæ, muriculatæ. (Paris).

\section{Gen. Goleochætium Besch.}

C. Renauldii Broth. in hb. (PI. xxu). - Luteo rufescens. Cæspites sat densi. Caulis repens, valde radiculosus irregulariter ramosus, rami 
turgidi 4-6 mil. longi subfastigiati. Folia humida incurvata pro more in uno latere dejecta, sicca valde crispata, sat dense conferta $2 \mathrm{mil}$. longa, o,8 mil. lata e basi ovata angustata acuta, longe hyalino decurrentia, marginibus basi et interdum ultra arcte revolutis, integris. Costa dorso lævi usque ad apicem producta. Rete obscuro, cellulis minutis hexagonoquadratis papillosis papillis dorso vix vel non prominulis, basilaribus ad margines haud dentiformi prominentibus ; alis decurrentibus hyalinis laxe textis e cellulis inflatis breviter rectangulis compositis; cellulis appendiculariis $8-\mathrm{I}_{2}$ maxime evolutis usque $75 \mu$ longis ellipticis vel obtuse conoïdeis molem distinctissimam efformantibus.

Madagascar: sine loco, (Hb. Brotherus).

Cette espèce se distingue immédiatement de ses congénères par une taille plus robuste et ses feuilles beaucoup plus grandes, plus fortement crispées à l'état sec, nullement contournées en spirale.

Elle differe du C. appendiculatum Ren. Card. par ses feuilles entières, lisses sur le dos ainsi que la nervure qui atteint le sommet où elle se dilate, par l'appendice de la décurrence beaucoup plus développé, enfin par les cellules papilleuses marginales de la base non saillantes en pointes dentiformes.

C. subappendiculatum Broth. in hb. (PI. xxir), - Luteo rufescens. Caulis repens valde tomentosus inordinate ramosus, rami graciles 4-6 mil. longi. Folia humida erecto-patentia apice interdum incurvata, sicca tortilla $1 \mathrm{r} / 4 \mathrm{mil}$. longa $\mathrm{I} / 2 \mathrm{mil}$. lata e basi ovata late et obtuse acuminata, interdum sublingulata longe hyalino decurrentia, marginibus hic illic præcipue ad basin arcte revolutis apicem versus minute erosodenticulatis, supra basin papillis dentiformi prominentibus, dentibus nonnullis acutis intermixtis, hirsutis. Costa sub apice evanida, dorso rugosa. Rete obscuro, papillis dorso acute prominentibus. Cellulis alaribus longe decurrentibus hyalinis elongate rectangulis passim subhexagonis, cellulis appendiculariis nullis vel rudimentariis irregularibus parce evolutis.

Madagascar : Volon Cazo, Leblanc, i 899, (Hb. Brotherus).

Cette espèce tient le milieu entre C.plicatum (P. B.) et C. appendiculatum Ren. Card. Elle differe du premier par sa couleur roussâtre, ses feuilles non contournées en spirale et plus fortement crispées à l'état sec, plus obtuses, longuement décurrentes et munies au-dessus de la décurrence hyaline de saillies dentiformes. taires.

Elle se distingue du second par les cellules appendiculaires nulles ou rudimen- 


\section{Gen. Macromitrium Brid.}

M. SANCte Marie Ren. Card.

Madagascar: Andevorante, 190I,(Hb. Paris). Nouveau pour la grande île.

M. subpungens Hpe.

Madagascar: Maroantsetra, baie d'Antongil, Mathieu (Hb. Cardot). Espèce des Comores, nouvelle pour Madagascar.

\section{Gen. Schlotheimia Brid.}

S. Microcarpa Sch.

Madagascar: province d'Ambositra, Anzorozoro, Frissard, 1905, (Hb. Paris).

\section{S. quadrifida Brid.}

Maurice: sine loco, PECK.

Cet échantillon que m'a communiqué le Dr Farlow de l'Université de Cambridge (Mass.) appartient au $S$. quadrifida Brid. L'incertitude qui régnait au sujet de l'existence de cette espèce dans les Mascareignes (cf. Prodrome, page ${ }_{151}$ ) cesse done aujourd'hui.

S. Trichophora Ren. Card. subsp. * S. stenophylla Ren. Card. A forma typica differt foliis longioribus, angustioribus, acutis vel minus obtusis, dorso vix corrugatis, pilo longiore instructis, basi plicis duobus notatis.

Madagascar: Betsileo! Ambositra? Rev. Villaume,(Hb. Lacouture).

Cette forme parai assez distincte du type pour qu'on soit tenté de la considérer comme une espèce propre, mais l'exiguïté de l'échantillon ne permet guère de trancher la question avec une entière certitude.

Dans le S. trichophora R. C. type, les feuilles raméales lingulées sont peu rétrécies dans la moitié supérieure, obtuses et souvent émarginées au sommet, l'excurrence pilifère sortant du sinus (les feuilles caulinaires seules acuminées, aiguës, cuspidées); elles sont fortement ondulées-plissées (corrugata) aux bords et sur le dos; en outre, les cellules apicales sont assez grandes, obliquement rhombées. Dans la subsp. stenophylla les feuilles raméales sont un peu plus longues ( 1,65 au lieu de 1,50 mil.) et un peu plus étroites rétrécies insensiblement dès le milieu,presque acuminées, dépourvues 
ou marquées seulement légèrement de rides ondulées et munies à la base d'un pli longitudinal situé, de chaque côté, à mi-distance de la nervure et de la marge; enfin les cellules apicales sont plus petites et carrées en séries droites. Enfin un autre échantillon de Madagascar, très grêle aussi, paraissant se rapporter à la même forme, montre des pédicelles très grêles, presque capillaires, longs de 8-10 mil. et des coiffes petites, longues de 2,5 mil., brunes, plus pâles à la base qui est 4-5 lobée, ovales, rapidement rétrécies, lisses et obtuses au sommet qui est arrondi, non apiculé. En attendant que des échantillons plus complets soient retrouvés, il est préférable de conserver au *S. stenophylla $\mathrm{R}$. C. le rang de subspecies.

S. (acuminella) acutifolia Ren. Par. (Pl. xıv). - Cæspites laxi inferne rufescentes, rubiginosi, superne virides. Caulis repens fasciculatoramosus, rami acuti parum divisi vel subsimplices, I5-20 mil. alti, vix tomentosi. Folia erecto-appressa, plerumque apice diffracta, superiora (juniora) viridia vix flexuosa haud spiraliter torta, parum conferta, dorso corrugata, e basi oblonga mox attenuata,lanceolata!longe angustata, acuta haud mucronata, magnitudine inæqualia, majora 3 r/2 mil. usque longa; costa flexuosa sub vel cum apice finiente, cellulis basilaribus elongatis oblique rectangulis, cæteris quadrato-hexagonis, 4-6 $\mu$ latis, sæpius - parietibus incrassatis - minoribus irregularibus subrhombeis. Fol. perich. longiora erecta. Capsula in pedicello 4 mil. longo erecta, cylindrica, 2 mil. longa sicca brunnea plicatula; calyptra $2 \mathrm{I} / 4$ mil. longa apiculata, lævis in lobis 4 latis truncatis brevibus fissa. Cætera ignota.

Madagascar: province de Betafo, forêt d'Ambohitantely, (Hb. Paris) 1901 .

Se distingue immédiatement du Schl. Boivini Besch. par le port plus grêle, les rameaux non gonflés, les feuilles moins serrées, dressées, nullement contournées en spirale, plus longues et plus étroites, longuement et nettement rétrécies acuminées, et par la coiffe lisse, de moitié plus petite. Ne peut d'ailleurs être confondu avec le Schl. quadrifida Brid. qui a le pédicelle 3-4 fois plus long.

S. (ligularia) Pauli Ren. Card. (Pl. xuI). - Cespites densi, late extensi, intus rufo-fuscescentes, superne plus minus virides. Caulis repens, valde radiculosus, ramis turgidis, apice obtusis, 10-1 2 mil. altis, superne fastigiato-ramulosis. Folia densissime conferta, sicca vix spiraliter torta, e majoribus generis, oblongo-ligulata, apice obtusiuscula vel plus minus attenuata, mucronata, basi angustata, vix corrugata, $2-2,25$ mil. longa, o,8 lata, marginibus planis, integerrimis, cellulis lævibus hexagonis, superioribus transverse dilatatis, inferioribus linearibus, lutescentibus, parietibus incrassatis, costa in mucronem brevem 
excedente. Folia perichætialia multo minora, I,5 mil. longa, oblonga, apice acutiuscula, mucronata. Capsula in pedicello tenui, flexuoso, pallide rufo, 5-7 mil. longo erecta, humida oblonga, I,5 mil. longa, collo distincto instructa, sicca plicatula sub ore leniter constricta. Exostomii dentes siccitate incurvati, $0,27 \mathrm{mil}$. longi, longitudinaliter papillose striati; 8-10-articulati. Cætera desunt.

Madagascar: Antsahambavy (alt. I 200-1 300 mètres), Dalincovrt.

Cette espèce paraît se rapprocher beaucoup du S. tenuiseta C. Müll. Toutefois, l'échantillon original de C. Müller est dépourvu de capsules mûres et la diagnose de cette espèce dans les Reliquice Rutenbergiance est si courte qu'il est difficile d'en conclure que la plante de C. Müller correspond entièrement à la nôtre, qui a, d'ailleurs, les feuilles plus étroites.

Notre espêce se distingue en outre: $\mathrm{I}^{\circ} \mathrm{du} S$. phacochlora Besch. par les feuilles beaucoup plus grandes, le pédicelle plus long, et la capsule oblongue, plus courte; $2^{\circ}$ du $S$. Perroti Ren. et Card. par les rameaux gonflés, obtus, par les feuilles plus grandes, presque lisses, à peine contournées en spirale à l'état sec, par les feuilles périchétiales non tronquées-émarginées au sommet, et par la capsule plus grosse, renflée à l'état humide.

S. microphylla Besch. subsp. ${ }^{*}$ S. Farlowii Ren. Card.-A planta typica madagassa differt habitu paulo graciliore, foliis duplo fere longioribus usque I mil. longis, ligulatis apice rotundatis, costa excedente brevissime apiculatis. Folia perichetialia multo majora $1,50 \cdot 1,65$ mil. longa, o, 5 mil. lata, erecta oblonga, acuminata, cuspidata, plicata, marginibus e medio involutis. Capsula in pedicello crassiusculo $3-4 \mathrm{mil}$. longo, oblongo-cylindrica, I mil. longa. Exostomii dentes extus reflexi, endostomii erecti. Calyptra parva, apice lævis, distincte apiculata.

Maurice: sine loco, ( $\mathrm{Hb}$. Farlow).

Les différences qui séparent cette race đe Maurice du S. microphylla Besch. de Madagascar, sans être bien importantes, me semblent cependant suffisantes pour l'admettre comme subspecies, surtout les feuilles plus longues, ligulées arrondies au sommet et les folioles périchétiales nettement aiguës, brièvement cuspidées par l'excurrence de la nervure.

D'autre part, il n'y a pas de confusion possible avec le $S$. squarrosa Brid. qui a un port plus élancé, les feuilles aiguës, le pédicelle plus long (6-7 mil.), la capsule ovale-oblongue pourvue d'un col distinct, enfin la coiffe renflée à la base, rapidement rétrécie et contractée au sommet en une pointe souvent allongée rostriforme. 
Gen. Splachnobryum C. Müll.

S. InUndatum Besch.

Madagascar: Cercle de Maintirano, Anosibe, (Hb. Paris).

N'était encore connu que de l'île de Nossi-Bé.

Gen. Orthodon Bory.

O. BORBonicus Bory.

Madagascar: Betsileo! Ambositra? Rev. Villaume,(Hb. Lacouture).

En belle fructification, mais sans coiffes.

\section{Gen. Entosthodon Schwgr.}

E. Renauldii Thér. in hb. (Pl. xxiti).

Synoïque. Tiges grêles allongées, généralement simples, 6-10 mil. Feuilles espacées sur la tige, les supérieures plus grandes rapprochées en rosette, oblongues-allongées, spatulées, aiguës, dentées depuis le milieu, marginées par 2 séries de cellules linéaires; dimensions des feuilles moyennes: 2 mil. sur 0,8 mil.; cellules rectangulaires ou hexagonales allongées, les inférieures plus longues, I $20 \mu$ sur $30 \mu$, les supérieures $60 \mu$ sur $25 \mu$, nervure mince, $48 \mu$, s'éteignant sous le sommet. Fleurs synoïques, anthéridies assez nombreuses, archégones rares, paraphyses courtes en massue. Pédicelle dressé, flexueux, pâle, long de 6-8 mil., épais de 0,14 mil. Capsule droite, brunâtre, petite, ovale ou oblongue, faiblement resserrée sous l'orifice, atténuée à la base en un col presque aussi long qu'elle, gymnostome; opercule plan-convexe; spores verruqueuses $20-25 \mu$. Coiffe inconnue. (Thériot in litt.).

Madagascar: Betsileo, Rev. Villaume, 1905, (Hb. Lacouture).

$E$. borbonicus Besch. s'en distingue aisément par le pédicelle 2 fois plus long, rouge, par la capsule plus grosse non défluente à la base et par l'inflorescence monoïque. 
E. mauritianus Schp. en est beaucoup plus voisin; il en diffère par sa faible taille, par ses feuilles plus petites, plus rapprochées, par le pédicelle plus court et plus épais et par ses cellules foliaires carrées. tome.

E. marginatus C. Müll. en est bien distinct par sa capsule pourvue d'un péris-

\section{Gen. Brachymenium Hook.}

B. Heribaudi Ren. Card. var. madagassum Ren. Card. - A planta borbonica differt cæspitibus læte vel pallide viridibus, densis, planis, sat longe extensis, tapetis ad instar, caule breviore, foliis multo minoribus $3 / 4$ mil. longis, minus acutis, marginibus subplanis, costaque pro more sub apice evanida.

Madagascar: Imerina, Rev: CAмвouḱ, I 906, (Hb. Lacouture).

Cette variété très distincte du type de la Réunion parait être la forme ordinaire des argiles rouges du plateau de l'Imerina.

B. Subflexifolium Ren. Card. var. pachyloma Ren. Card. - A planta typica differt foliis paulo minoribus, basi angustatis, limboque crassiore, latiore, e cellulis 4-5 formato. Capsula in pedicello flexuoso $25 \mathrm{mil}$. longo erecta, ovata raptim in collum corrugatum coarctata, matura brunnea, ore rubello, interdum pedicelli curvatura subpendula. Peristomii vetusti dentes externi breves obtusi, membrana interna maxime granulosa. Flores masculi in ramo proprio basilari complures.

Madagascar : Betsileo, Rev. Villaume, I9o5-o6, (Hb. Lacouture).

Cette variété forme une transition vers le $B$.nigrescens Besch. De nouvelles observations semblent nécessaires pour mieux délimiter ce dernier vis-à-vis du $B$. subflexifolium Ren. Card. et même du B. capitulatum Mitt. dont je ne connais le type que par la description de l'auteur et dont les échantillons malgaches se distinguent tout d'abord par l'absence ou la brièveté des innovations, lesfeuilles enroulées en spirale, non flexueuses et le pédicelle un peu plus court, tandis que dans le B. subflexifolium R. C. toutes les tiges s'allongent par des innovations grêles munies de feuilles étroites, flexueuses au subcrispulées. Ses touffes lâches sont d'un rouge vineux à l'intérieur et vertes à la surface.

B. Philonotula Hpe var. purpurellum Ren. Card. - A planta typica differt caule brevissimo subnullo, foliis in comam sphæricam congestis, pedicello breviore intense purpureo capsulaque ventricosa pulchre sanguinea. Forsan species propria. 
Madagascar : Fianarantsoa, Rev. Villaume, 1905, (Hb. Lacouture).

Cette forme est tellement différente du type dont les innovations plus ou moins allongées portent des feuilles légèrement homotropes, qu'à première vue on est tenté de la considérer comme une espèce propre.

B. lineale Ren. Card. - Tenellum; caulis pergracilis, subsimplex, 5-10 mil. altus, tota fere longitudine denudatus vel squamose foliosus. Folia comalia conferta, erecto-patentia, lineali-lanceolata, 3,5 mil. longa, elimbata, cellulis mediis lineari-rhombeis, 8-1o-longioribus quam latioribus, superioribus angustioribus linearibus. Capsula in pedicello rufo anguste cylindrica, basi longe attenuata, ore truncata, 2-3 mil. longa. Peristomii externi dentes pallidi, o, 26 mil. longi, tenuiter trabeculati, superne papillosi ; membrana interna ad medium vel paulo infra producta; processus integri, aequilongi, granulosi, marginibus erosi. Cilia...? Sporæ granulosæ, diam. I6-20 $\mu$.

Madagascar: mont Anteby, près Ambositra, Dr Forsyth Major, (Hb. Levier).

Espèce très distincte de toutes celles de la région par le port, qui rappelle celus des petites formes grêles du Webera elongata. Elle ne peut, d'autre part, être confondue avec le Brachymenium coarctatum Bryol. jav., de Java et de l'Océanie, avec lequel elle n'a qu'une ressemblance extérieure.

\section{Gen. Epipterygium Lindb.}

E. diversifolium Ren. Par. - E minimis, E. Tozeri europæo aliquantulum simile. In terra humida inter Algarum tapetem gregarie vel sparse crescens. Caulis debilis $4-8$ mil. altus basi parce purpureo radiculosus. Folia valde diversiformia, inferiora remota, patula, vinosula, late ovata apice rotundata subcohleariformia, superiora pallide viridia confertiora laxe imbricata longiora (I mil.) elongato-oblonga obtuse acuminata, comalia in gemmam conniventia, omnia valde concava, marginibus integerrimis late inflexis. Costa pertenuis deplanata, interdum obsolete notata longe ante apicem dissoluta. Rete perlaxo hyalino: cellulis fere conformibus subinanibus, basilaribus vix brevioribus, mediis elongate subhexagonis 3 long. $(90-100 \mu)$ quam lat. $(25-30 \mu)$ marginalibus interdum angustioribus sed haud limbum efformantibus. Cæetera desunt.

Madagascar: Cercle de Maintirano, ad moles tophaceas, 1902, (Hb. Paris). 
Cette espèçe ne peut être confondue avec E. convallium Dusen (E. Duseni C. M.) du Cameroon, dont le port et la forme des feuilles sont très différents. Elle a plus de rapports avec $E$. Tozeri d'Europe, mais elle s'en distingue facilement par ses feuilles dépourvues de margo.

Gen. Bryum Dill.

B. (argyrobryum) Leloutrei Ren Card. - Cespites dense pulvinati, laete vel glauco-virides, intus tomento radiculoso ferrugineo valde intertexti. Caulis 5-8 mil. altus, superne fasciculato-ramoșus, ramis turgide julaceis. Folia arcte imbricata, late ovata, 0,75-1 mil. longa, cochleariformi-concava, obtusa vel brevissime acuminata, marginibus pro more involutis, summo inciso-denticulatis, costa tereti viridi in aristam subcequilongan remote dentatam, erecto-patentem vel subsquarrosam excurrente; cellulis omnibus viridibus, chlorophyllosis, inferioribus quadratis vel breviter rectangulis, mediis hexagonis, 2-3 long. quam lat. superioribus paucis brevibus, marginalibus incrassatis, angustis, scariosis, limbum subefformantibus. Perichætiala in aristam longìssimam remote dentatam producta. Cætera desunt.

Madagascar: montagne d'Ambre près Diego Suarez, alt. 800-10oo mètres, Leloutre, 1898 ( $\mathrm{Hb}$. Corbière).

Comparable au B. arachnoideum C. Müll. de Kitui (Afrique tropicale occidentale), mais distinct par son port plus robuste, ses coussinets d'un vert glaucescent envahis à la base par un tomentum ferrugineux très abondant, par les feuilles plus obtuses et dentées-incisées au sommet, où elles sont submarginées, brusquement et plus longuement aristées, par les cellules vertes; toutes chlorophylleuses, les apicales courtes; les marginales supérieures rendues très étroites par l'épaississement des parois longitudinales et simulant un margo.

Dans le B. arachnoideum C. Müll., les cellules du quart supérieur de la feuille sont allongées, délicates, hyalines.

B. Leptospeiron C. Müll.

Madagascar: province de Tamatave, forêt de Fito, PERRot, I897, (Hb. Levier).

B. subgracilescens Ren. Par. - Cæspites molles pallide virides. Caulis fastigiatus subsimplex flexuosus, elongatus, gracilis, erectus, circa 4 cent. longus, basi rufescens superne vìridis. Folia remotiuscula, 
inferiora decolorata erecto-patentia, sicca flexuosa, nonnunquam spira liter torta, usque $5 \mathrm{mil}$. longa, oblongo-lanceolata, acute acuminata, longe decurrentia, haud spatulata, costa breviter excedente, marginibus dimidio inferiore arcte revolutis superne planis et spinoso dentatis, cellulisque marginalibus sensim angustioribus $3-4$ seriatis veluti limbatis. Cellulis basilaribus vacuis rectangulis vel hexagono-rectangulis, mediis et superioribus elongate subhexagonis $60-70 \mu$ longis, utriculo primordiali plus minus conspicuo. Cætera desunt.

Madagascar : province de Moramanga, forêt d'Analamazaotra, (Hb. Paris).

Par le port, cette espèce rappelle un peu la forme grêle du $B$. spinidens $\mathrm{R}$. C. de Fianarantsoa (cf. Ren. Prodr. f. Mad., etc. p., i66), quoique les feuilles soient moins grandes et moins étalées; mais ce dernier a les feuilles du double plus longues, les cellules plus amples, les marginales supérieures ne formant pas de margo. Par la forme des feuilles et le tissu, le $B$. subgracilescens se rapproche du B. truncorum Bory; mais celui-ci a un tout autre port, une tige courte, les feuilles réunies en rosette comale, munies de dents beaucoup plus faibles.

B. erythrocaulon Schwgr.

Madagascar: Betsileo, Rev. Villaume, 1906, (Hb. Lacouture).

B. suberythrocaulon Ren. Card. - Cæspites sat densi, superne pallide virides inferne nigricantes. Caulis robustus, fastigiatus, simplex, rarius apice divisus, erectus, 4-6 cent. altus, tota fere longitudine foliosus, fusco radiculosus. Folia æequaliter conferta comalia haud rosulata, humida erecto patentia, sicca subappressa, complanata vel vix flexuosa, haud spiraliter torta, 5 mil. longa, oblongo-ligulata medio haud latiora, acuminata; marginibus e basi usque ad 5/6 revolutis apice planis, superne acute sed breviter serrulatis; costa in cuspidem brevem obliquam excedente. Cellulis vacuis parce granulosis, basilaribus rectangulis, marginalibus angustissimis, linearibus, incrassatis 4-5 seriatis, limbum flavidum efformantibus.

Madagascar : Fianarantsoa, in locis humidis, Dr Besson, I $89 \mathrm{I}$.

Cette espèce se distingue à première vue du B. erythrocaulon Schwgr. par son port plus robuste, sa taille plus élevée, ses touffes entièrement formées par des inno. vations sans rosette comale, par ses tiges robustes raides envahies par un feutre de radicules noirâtres, par ses feuilles plus larges, aplanies, nì flexueuses, ni contournées en spirale à l'état sec, non rétrécies à la base quoique longuement décurrentes, non élargies au milieu, à marges presque parallèles, révolutées dans presque toute 
la longueur, planes seulement au sommet. Le margo est très large et composé de cellules linéaires subvermiculaires à parois très épaissies. La serrature se compose de dents aiguës, courtes, à peu près comme dans le $B$.truncorum Bory, beaucoup moins fortes que dans les $B$ appressum $\mathrm{R}$. C. et subappressum R. C., à plus forte raison que dans le $B$. spinidens $\mathrm{R}$. C.

B. Appressum Ren. Card. var. Forsythii Ren. Card. - A planta typica differt foliis minus appressis latioribus, costa oblique excurrente abruptius apiculatis, denique limbo duplo latiore e cellulis 6-8 seriatis efformato. Forsan species propria.

Madagascar : forêt d'Ambohimitombo (Tanala), D' Forsyth MAJor, (Hb. Levier).

B. angustissimifolium C. Müll. - Delicatulum, laxe cespitosum, saturate viride. Caulis gracilis, circa 15 mil. altus, subsimplex, inferne vix denudatus. Folia remotiuscula, e basi longe angustata, imbricata, decurrente, humida extus recurvata, subsquarrosa, sicca pro more spiraliter torta, medio latiora, anguste lanceolata, sensim cuspidata, 3,5 mil. longa, costa excurrente, marginibus e basi ad medium vel ultra revolutis, superne planis, denticulatis, cellulis basilaribus vinosulis, elongate rectangulis, nonnullis hexagonis, mediis hexagono-rhombeis, utriculo primordiali moniliformi repletis, marginalibus sensim angustioribus et longioribus, limbum angustum ( $35 \mu$ latum, e cellulis 2-4seriatis) interdum ægre limitandum efformantibus. Cætera desunt.

Madagascar : Fenerive, (Perrot ; Hb. E. Levier).

Espèce caractérisée par son port un peu grêle, ses feuilles étroites, étaléessquarreuses à l'état humide et tordues en spirale à l'état $\mathrm{sec}$, ce qui la distingue des autres espèces de la section Eubryum habitant la région malgache.

B. homalobol.Ax C. Müll.

Madagascar: province de Tamatave, forêt de Fito, Perrot, I897, (Hb. Levier); entre Fianarantsoa et Mananjary, I891, Dr Besson ; province d'Antsirabe, Galinon, 1903 (Hb. Paris); Betsileo, Rev. Villaume, 1905, (Hb. Lacouture); paraît assez répandu dans cette dernière province; cercle d'Ivondro, pays des Baras (Hb. Paris).

B. subhomalobolax Ren. Card. - Habitu B. subappresso R. C. sat simile. Caulis brevis I $1 / 2$ cent. altus denudatus inter cespites Brachythecii atro theca Dub. sparse crescens. Folia in gemmam apica- 
lem dense conferta, intense viridia subspiraliter contorta 3-3 1/2 mil. longa, lata ligulata vel subspatulata apice rotundata vel latissime rotundato-acuminata; marginibus inferne revolutis superne planis et denticulatis, limbo angusto e cellulis $1-2$ seriatis, superne dissoluto. Costa in mucronem rectum breviusculum exeunte. Cellulis omnino utriculo primordiali repletis, breviter subhexagonis apicalibus vix longioribus quam latioribus, basilaribus rectangulis, vacuis.

Madagascar: Fianarantsoa, Rev. Villaume, i go6, (Hb. Lacouture).

Cette plante ressemble parle port au B. subappressum R. C., mais s'en distingue par la forme des feuilles plutôt arrondies au sommet qu'acuminées et par le mucron droit plus court, formé par l'excurrence de la nervure. Il differe d'ailleurs du $B$. homalobolax C. M. dont il serable être une réduction, par sa très petite taille, par ses feuilles très arrondies au sommet et par la brièveté des cellules, les apicales à peine plus longues que larges.

Le B. truncorum Bory des Mascareignes ne peut être confondu avec notre plante à cause de ses feuilles plus longues, acuminécs et largement marginées.

B. Alpinulum Besch. var. densum Ren. Card. - A planta typica Nossibeana differt cæspitibus compactis extensis maxime cohærentibus, viridibus, foliis magis appressis, distinctius revolutis, costaque luteoviridi haud rubella sæpius leviter excedente.

Madagascar: Imerina, Ambohibeloma, Rev. Cambout, rgo6, (Hb. Lacouture).

Une autre forme (var. Levieri R. C.), collectée par Perrot dans la forêt de Fito (Hb. Levier) et que je rapporte au B. alpinulum Besch., a les feuilles beaucoup plus grandes, plus larges, surtout, que le type de Nossi-Bé, légèrement courbées asymétriques, à marges jnvolutées dans la moitié supérieure jusqu'au sommet qui est cuspidé par une légère excurrence de la nervure, tandis que dans le type, les marges supérieures sont planes et concourent avec la nervure à former l'acumen un peu obtus. Le tissu est le même.

Le B. nanopyxis C. Müll. de Nossi-Bé (Hildebrandt) nomen midum, ne me paraît pas différer de $\boldsymbol{B}$. alpinulum Besch.

B. Bessoni Ren. Card. - Cæspites densi obscure virides purpureo variegati. Caulis erectus, rigidus, simplex, fastigiatus, vix $10 \mathrm{mil}$. altus. Folia dense conferta appressa anguste lanceolata, sensim angustata interdum subligulata acuminata, apice crenulata; marginibus usque fere ad apicem revolutis; costa crassa rufa breviter excedente. Rete sat denso, cellulis vacuis mediis rhombeis, basilaribus quadratis vel breviter rectangulis. Cætera desunt. 
Madagascar: Fianarantsoa, in locis humidis, Dr Besson, 1892.

Cette espèce voisine du B.alpinulum Besch. en diffère essentiellement par ses feuilles plus grandes, par le tissu dense à cellules courtes et par la nervure plus longuement excurrente. En outre les touffes sont plus denses, les tiges raides, les feuilles plus serrées appliquées, crénelées au sommet. C'est, comme port, une réduction du B. alpinum L. d'Europe.

D'autre part, on ne peut confondre cette espèce avec $B$. leptospeiron C. Müll. de la même région dont la taille est analogue, mais dont les feuilles sont contournées en spirale à l'état sec et d'une tout autre forme.

\section{Gen. Mnium L.}

M. Australe Besch.

Madagascar : Fianarantsoa, Dr Besson, 1892 ; Betsileo, Rev. VILLAUME, I 905 , (Hb. Lacouture).

\section{Gen. Philonotis Brid.}

P. obtusata C. Müll.

Madagascar: province de Betafo, Mianadantsola, 1901,(Hb. Paris); Ambohibeloma, Rev. Cambout, i go6, (Hb. Lacouture).

P. Bescherellei Thériot in litt. (Philonotis perigonialis Besch. ex parte).

"Dioüque. Tige de I-2 cent. rameuse. Feuilles caulinaires des tiges stériles lancéolées, assez largement acuminées, étroitement révolutées aux bords jusqu'aux $3 / 4$, à marge denticulée par les papilles dans la partie révolutée et dentée dans la partie plane, longues de I-1,2 mil., larges de 0,4 mil. Tissu opaque papilleux; une grosse papille élevée, arrondie, placée sur le lumen de la cellule et non au sommet; cellules inférieures rectangulaires $12-18 \mu$ sur $9 \mu$. Nervure excurrente, dentée-papilleuse de la base au sommet, large de $3 o \mu$.

Plante mâle: Feuilles caulinaires un peu plus larges et moins longuement acuminées que dans la tige stérile; fleur terminale discoïde, folioles périgoniales très grandes $2-3$ fois plus longues que les caulinaires, très étalées, parfois même squarreuses, acuminées, à peu près planes aux bords, très concaves à la base ; nervure excurrente, cellules inférieures rectangulaires, lisses, les suivantes papilleuses à papille située à l'angle supérieur de la cellule ; paraphyses très longues, clavitormes• Plante femelle inconnue.

La Réunion: LÉpervanche, I 877. (Hb. Bescherelle). 
A la suite de la description de Ph. pertgonialis Besch., Bescherelle cite comme représentant son espèce 2 plantes collectées à La Réunion, l'une par Frappier, l'autre par Lépervanche. En réalité ces deux plantes sont tout-à-fait distinctes; elles ont de commun la forme de la fleur mâle et le développement inusité des folioles périgoniales, mais elles diffèrent entièrement pour tout le reste. Bescherelle adonc confondu deux espèces sous le même nom de $P$. perigonialis $B$. Or, comme la description du $P$. perigonialis convient seulement à la plante de Frappier, que d'ailleurs c'est bien celle-ci que Bescherelle a eu en vue puisqu'elle est la seule qui soit fructifiée et qui figure sous le nom de $P$. perigonialis dans l'herbier des types légué par Bescherelle au Museum de Paris, on doit conserver le nom donné par Bescherelle à la plante récoltée par Frappier et celle de Lépervanche prendra le nom de $P$. Bescherellei Thér.

Le $P$. Bescherellei se distingue aisément de $P$. perigonialis par ses feuilles deux fois plus courtes et par la disposition toute particulière des papilles qui sont situées sur le milieu de la cellule et non sur la cloison intermédiaire.

A ces observations de M.Thériot j'ajoute que l'échantillon original de La Réunion que j'ai reçu de Bescherelle, sans nom de collecteur nide localité, se composait de 2 uniques tiges, l'une stérile, l'autre mâle. Je ne pouvais soupçonner, à priori, que ces tiges ne provenaient ni du même collecteur, ni de la même localité, et j’avais pensé que, dans cette espèce, les feuilles des tiges mâles n'étaient pas identiques à celles des tiges femelles, stériles ou non. L'examen fait par M. Thériot de matériaux plus complets tranche la question et prouve qu'il y a eu en réalité confusion de 2 espèces différentes, dont l'une: $P$. Bescherellei Thér. s'éloigne plus de ses congenères que le $P$. perigonialis Besch., celle-ci très voisine de $P$. submarchica Besch.

Il y aura lieu d'ailleurs de réduire le nombre des espèces de Philonotis de notre Domaine. C'est ainsi que les $P$. comorensis C. Müll, et $P$. caspitans C. Müll. ne peuvent être séparés spécifiquement du laxissina $\quad$ C. Müll $\left({ }^{*}\right)$. Les différences sont établies sur le port et la ramification, et l'on sait combien ces caractères sont peu stables chez les Philonotis, mais il n'y a aucune divergence anatomique appréciable. Le $P$. tenuicula Hpe ne parait être qu'un "forma depauperata " du même type général.

Le $P$. mauritiana Angstr. a une taille plus élevée et les feuilles un peu plus grandes, mais le reste concorde, et d'ailleurs mon ami Cardot et moi avons constaté à Madagascar des formes de transition entre cette espèce et le $P$. laxissima C. M. De même, d'après $M$. Thériot, le $P$. luteo viridis Besch. ne diffère du $P$. mauritiana Angstr. que par des caractères légers et il fait observer en outre que les $P$. curvifolia Besch. de La Réunion et $P$. sparsifolia Hpe sont extrêmement voisins.

\section{Gen. Pogonatum P. Beauv.}

P. Macei Thériot, in litt.

"Tige dressée haute de 20 mil. Feuilles inférieures et moyennes espacées, courtes, appliquées sur la tige, les comales plus longues (5 mil.) formant touffe, dressées appliquées à l'état sec, non crispées,

* M. Fleischer est arrivé à la même conclusion pour lé $P$. comorensis C. M. 
étalées à l'état humide, à gaîne courte, large, ovale, à lame à bords relevés, entiers, acumen subobtus. Lamelles 40 composées de 6 séries de cellules, la terminale dilatée transversalement à bord libre, plein ou arrondi. Cellules de la partie vaginante carrées, les inférieures près de la nervure brièvement rectangulaires, cellules des bords de la lame carrées, 20-24 $\mu$. Pédicelle long de 15 mil. Capsule cylindrique longue de 4 mil. ». (Thériot in litt).

Grande Comore : volcan du Kortala (alt. 600 mètres) Macḱ, 1900, (Hb. de Poli).

" Cette espèce paraît bien distincte de toutes celles connues dans les îles austroafricaines de l'Océan indien ».

P. Parisij. Thériot, in litt.

“ Tiges courtes, I cent. au plus. Feuilles molles dressées-étalées à l'état sec, non crispées, planes, lancéolées-ligulées, non dilatées et peu engainantes à la base (gaine très courte) dentées immédiatement audessus de la partie hyaline, longues de 4 mil.; lamelles 40 , peu élevées à 3-4 séries de cellules, la terminale arrondie, dilatée, lisse; cellules de la base généralement carrées, les suivantes petites, longues de $9-12 \mu$, dilatées transversalement. » (Thériot in litt.).

Nossi Comba: Marie (Hb. Bescherelle).

" Cette espèce rapelle par le tissu foliaire, les lamelles et la denticulation le $P$. brachythecium Besch. de La Réunion; mais elle s'en distingue à première vue par le port, les feuilles planes, non crispées et la gaine très courte ».

\section{CLADOCARPI}

\section{Gen. Cryphæa Mohr.}

C. Rutenbergir C. Müll.

Madagascar: Moramanga, Door, I897, alt. 1000 mètres.

M. Brotherus a reconnu que l'échantilion original de Renauldia dichotoma C. Müll. malgré sa taille élancée et son port spécial doit être identifié avec $C$. $R u$ bergii. Il considère aussi comme vraisemblable l'identité de ce dernier et du $C$. laxifolia. Mitt. de l'Usagara. 


\section{C. (acrocrypliara) subintegra Ren. Card.}

M. Brotherus pense que cette espèce est à peine distincte spécifiquement de C. Madagassa C. Müll. Lorsque nous l'avons fondée nous n'avions pu examiner d'échantillon de ce dernier que nous ne connaissions que par la description qui se réduit à ces quelques mots : "A præcedente (C. Rutenbergii C. M.) differt foliis integerrimis . Or, si dans le $C$. subintegra les feuilles des rameaux julacés sont entières, celles d'autres rameaux sont moins imbriquées et distinctement dentées au sommet, (voir l'Atlas des Mousses de Madagascar, Planche 65).

\section{Gen. Erpodium.}

E. (Leptocalpe) madagassum Par. Ren. (Pl. xvi),- Glauco-viride. Caulis repens radiculis corticibus arcte adfixus, dense ramulosus, rami brevissimi, erecti, turgídi. Folia tereti-imbricata, humida erecta, sicca arcte adpressa, ovato-rotundata vel subcircularia, nonnunquam apice paululum attenuata, obtusata vel brevissime apiculata, 3/4-1 mil. longa, ecostata, marginibus planis, integerrimis. Cellulis plus minus, sæpe vix chlorophyllosis, apicalibus exacte hexagonis (diam. I6 6 ), mediis et basilaribus paulo majoribus subhexagonis transverse dilatatis, oblatis 36$40 \mu$ latis), maginalibus paulo minoribus transverse rectangulis. Fructus ex apice ramuli oriundus; fol. perich. decolorata, scariosa, thecam omnino includentia, arcte adpressa, caulinis duplo majora, 2 mil. longa, late ovata, apiculata vel parce attenuata e cellulis vacuis subelongate hexagonis apicibus acutis (56-58 $\mu$ longis) reticulata. Capsula immersa subsessilis, robusta, ovato-oblonga, I $3 / 4 \mathrm{mil}$. longa, leptodermis, ore lato plicatulo interrupte laciniato, laciniis valde promimentibus. Annulus persistens peraltus, passim subduplex, e cellulis elongatis usque $70 \mu$ longis compositus. Operculum et calyptra desunt.

Madagascar : province d'Antsirabe, vallée de la rivière Andrantsay, Galinon (Hb. Paris).

Genre nouveau pour Madagascar. Il serait utile de comparer cette plante à $l^{\prime} E$. grossirete C. M. de la région zambézienne, dont nous ne possédons pas d'échantillons. La description de C. M. concorde en partie avec notre mousse; cependant il n'y est pas fait mention de cellules foliaires dilatées transversalement, et ce qui est dit de la capsule "theca minuta ", tandis que dans notre espèce elle est relativement très grosse, inspire des doutes sur l'identification possible des deux plantes. 
Gen. Braunia Bryol. eur.

B. entodonticarpa C. Müll.

Grande Comore: pentes du volcan Kortala, alt. 600 mètres, MAcÉ, I 900 , (Hb. de Poli).

Espèce trouvée d'abord au Kenia dans l'Afriquè équatoriale orientale. Genre nouveau pour notre Domaine.

\section{PLEUROCARPI}

Je crois utile d'indiquer ici, les déplacements génériques des espèces de notre Domaine, surtout en ce qui concerne celles de la famille des Neckéracées, afin d'établir la concordance avec les nouveaux genres fondés par MM. Brotherus et Fleischer.

Gen. Pterobryopsis Fleisch.

P. patentissima $(\mathrm{Hpe})=$ Garovaglia in Prodr.

Gen. Orthostichopsis Broth.

O. sublivens (Besch.), O. longinervis (R. C.), O. chrysoneura (Hpe), O. subimbricata (Hpe), O. debilinervis $(\mathrm{R}$. C. $)=$ Pilotrichella, O. scaberula (R. C.) = Porotrichtm in Prod.

Gen. Squamidium Broth.

s. biforme $(\mathrm{Hpe})=$ Pilotrichella in Prodr.

Gen. Aerobryopsis Fleisch.

A. subpiligera (Hpe), A. pseudo capensis (C. M.), A. crispicuspis $($ Besch $)=$ Aerobryum in Prodr. 
Gen. Aerobryidium Fleisch.

A. lanosulum (C. M.) = Aerobryum in Prodr.

Gen. Floribundaria Fleisch.

F. floribunda (D. M.), F. fulvastra (Besch.), F. floribundula (C. M.), F. patentissima (C. M.) = Papillaria in Prodr.

Gen. Trachypus R. et Hsch.

T. lætus (R.C.), T. appendiculatus (R.C.) $=$ Papillaria in Prod.

Gen. Trachypodiopsis Fleisch.

T. serrulata $($ Brid. $)=$ Trachypus in Prodr.

Gen. Galyptothecium Mitt.

C. acutifolium (Besch.) $=$ Neckera in Prodr., C. Bescherellei $($ Kiær. $)=$ Garovaglia in Prodr.

Gen. Homalia Brid.

H. pygmæa (R. C. $)=$ Neckera in Prodr.

Gen. Pinnatella (C. M.) Fleisch.

P. tamariscina (Hpc), P. Geheebii (C. M.), P. Chenagoni (C. M.) = Porotrichum in Prodr.

Gen. Campylodontium Doz. Molk.

C. mauritianum (C. M.) = Lepyrodon in Prodr.

Gen. Trachyphyllum Gepp.

T. fabronioides (C. M.), T. Ferriezii (Besch.), T. Borgeni (Kiaer), T. dilatatum $($ Ren.) $=$ Leptolymenium in Prodr. 
Gen. Cyclodictyon Mitt.

C. Hildebrandtii (C. M.), C. Auberti (P. B.), C. borbonicum (Besch.) = Hookeria in Prodr.

Gen. Haplohymenium Doz. Molk.

H. tenuissimum (Besch $=$ Pseudoleskea in Prodr.

Gen. Rauia Mitt.

R. subfilamentosa (Kiaer) $=$ Pseudoleskea in Prodr.

Gen. Pterogoniella Schimp.

P. Madagascariensis Brid.

Madagascar : Andevorante, ad citrum, igor, (Hb. Paris).

P. obtusifolia Ren. Card. - La fructification de cette espèce était encore inconnue, et la description doit en être complétée comme il suit:

Folia perich. lanceolata, integerrima, acuta, concaviuscula, superne marginibus involutis, evidenter costata. Capsula pallide lutescens leptoderma vix I mil. longa, sicca sub ore valde strangulata, madida ovata, in pedicello flavido 7-7 I/2 mil, alto, tortili, lævi erecta. Vaginula ovatooblonga archegoniis vestita. Perist. dentes externi madidi conniventes, sicci intus convoluti, fusci, trabeculati, intus lamellosi, obtusi, linea divisuriali vix perspicua, apice hyalino granulosi; processus papillosi medio angustissime fissi dentibus subæquilongi in membrana $\mathrm{r} / 3$ dentium æquante positi, sicci inter dentes erecti. Sporæ minutæ, luteæ, læves. Cætera desunt. (Paris).

Madagascar: Ankazobe, Valalafotsy, C. fr., Igo I, (Hb. Paris).

P. crassiretis Ren. Card. - Monoica, pallide vel lutescentiviridis. Caulis procumbens, circa I cent. longus, teres, turgide foliosus, parce ramosus. Folia arcte imbricata, concava, plicata, circa I mil. longa, ovato-oblonga, brevissime acuminata, omnia ecostata, margini- 
bus integris, late revolutis; rete incrassato, cellulis superioribus parvis, irregulariter subrotundis, parietibus sinuosis, mediis angustis, oblongolinearibus, supra-angularibus oblique seriatis, sublunatis, basalibus angulos versus 5-6, dilatatis, subvesiculiformibus, hyalinis vel luteis. Folia perichætialia externa brevia, intima oblonga, longius acuminata, integra. Capsula in pedicello brevi, 3-6 mil. longo, arcuato, erecta inclinatave, I mil. longa, oblonga. Peristomium simplex, dentibus erectis, albidis, angustis, 0,2 mil. longis, granulosis, obsolete trabeculatis. Operculum e basi convexa oblique longe subulatum. Calyptra cucullata apice rugosa. Sporæ magnæ, hirsutæ, 23-26 $\mu$ crassæ.

Madagascar : sine loco (Hb. Museum Paris).

Cette Mousse voisine de $P$. madagascariensis (Brid.) se distingue de l'échantillon original de cette espèce, rapporté par Dumont d’Urville de Madagascar, par ses feuilles plus serrées, plus étroitement imbriquées et surtout par le tissu foliaire composé de cellules à parois très épaissies, les moyennes étroites, sublinéaires, les suprabasilaires vers les angles très obliques, à parois flexueuses.

P. (?) undulata Ren. Card. - Pallidis viridis. Caulis repens, 3-4 cent. longus, ramis dense confertis, simplicibus, fastigiatis, crassiusculis, brevibus circa 5 mil. longis, erectis. Folia humida erecto-patentia, sicca subimbricata, oblongo-ligulata, subito breviter acuminato-apiculata, I mil. longa, o, 45 mil. lata, concava, interdum plicis duobus curvatis notata, marginibus e medio dentatis vel serrulatis (dentibus remotis, irregularibus, interdum patulis) apicem versus inflexis, undulatis, unde passim apiculo semitorto. Costa gemella longiuscula, nonnunquam simplici ad medium fere producta. Rete denso pallido, cellulis basilaribus linearibus, mediis longissime rhombeis sublinearibus, apicalibus brevioribus flexuosis, obtusis, parietibus incrassatis, omnibus lævibus, sed apice minute prominulis, alaribus paucissimis brevioribus et latioribus hexagono-quadratis ad angulos rotundatos haud excavatos auriculas minutissimas sistentibus.

Madagascar : forèt d'Analamazaotra, Rev. ChMbové, I8go.

Cette espèce très voisine de P. (?) fallax R. C. de Diego Suarez s'en distingue par ses rameaux très courts, épais, simples, par ses feuilles nullement homotropes, à bords fortement ondulés au sommet par l'inflexion irrégulière des marges qui occasionne souvent une tortion de l'apicule. En outre les nervures sont plus longues et parfois remplacées par une seule branche atteignant le milieu.

Le même doute qu'il est difficile de dissiper en l'absence de fructifications, plane toujours sur la place générique de ces 2 especes. Par le port et la forme des feuilles, 
elles ressemblent beaucoup aux Pterogoniella; mais le tissu est plus dense que dans ce dernier genre et les marges sont dentées. Sous plusieurs rapports elles se rapprochent des Camptochate et c'est même sous ce dernier nom (avec doute) que le $P$.fallax R. C. est figuré dans l'atlas des Mousses de Madagascar.

\section{Gen. Prinodon C. Müll.}

P. ciliatus Besch.

Grande Comore: pentes du volcan Kortala, MAcÉ, Igoo (Hb. de Poli).

Gen. Hildebrandtiella C. Müll.

H. endotrichelloides C. Müll.

Madagascar : forêt d'Analamazaotra, Rev. Caniboué, i 890.

Grande Comore: pentes du Kortala, I9o, Mact́, (Hb. de Poli).

H. rotundifolia Ren.

La Réunion: sine loco (Hb. Bescherelle). Voir Prodrome page 296.

\section{Gen. Papillaria C. Müll.}

P. BoIvini Besch.

Grande Comore: pentes du Kortala, Macé, I90o (Hb. de Poli).

P. africana C. Müll.

Madagascar: province de Betafo, Antsirabe, igo I, (Hb. Paris).

Le $P$. Ankeriensis Kiaer, Prodrome, page I92, ne paraît pas différer de $P$. afiricana C. M. ; il y a donc lieu d'ajouter à la localité d'Antsirabe, celles citées dans le Prodrome pour le $P$. Ankeriensis Kiaer.

Gen. Floribundaria C. Müll.

F. floribunda (D. M.).

Le $F$. fulvastra Besch. des Mascareignes n'est décidément pas assez distinct spécifiquement du $F$. floribunda D. M. Ce dernier, comme la plupart des espèces dont 
l'aire de dispersion est très vaste, subit quelques légères modifications quí autoriseraient tout au plus la distinction de races régionales ou sous-espèces. C'est donc avec raison que $\mathbf{M}$. Fleischer propose de fondre les 2 espèces.

Var. madagassa Ren. Card. - A planta typica borbonica differt foliis majoribus basi latioribus abruptius angustatis, longius acuminatis et rete basilari præcipue ad angulos, laxiore.

Madagascar : plateau d'Ikongo ; entre Sarondronina et Ranomafana; Fianarantsoa; entre Vinanintelo et Ikongo, $D^{r}$ Besson 1892 ; forêt d'Ambohimitombo, Dr Forsyth Major, (Hb. Levier).

Cette variété,qui paraît répandue à Madagascar dans la zône des forêts,se rappro. che parfois tellement des formes grèles du $F$. pseudo fulvastra C. M. ( $F$. patentissima C. M.) que dans certains cas l'attribution des échantillons à l'une plutôt qu'à l'autre des deux espèces devient difficile.

\section{F. patentissima (C. Müll.)}

Cette espèce de l'ile de Saint-Thomé est tellement voisine du $F$. pseudo fulvastra C. M., comme je l'avais déjà constaté dans le Prodrome (page 195) qu'il semble préférable de les réunir, spécifiquement, ainsi que le propose M. Fleischer. Le DrC. Müller m'a communiqué de Saint-Thomé des formes très grèles peu distinctes de la var. madagassa R. C. du F. floribunda. D'autre part se trouve à Madagascar (Fianarantsoa, Dr Besson; Betsileo, Rev. Villaume) une forme spéciale si différente du type qu'on la prendrait à première vue pour une espèce propre et qui est caractérisée de la façon suivante:

Var. squarrosa Ren. Card. - A planta typica differt : cæspitibus densioribus, caulibus minus elongatis, crassioribus, densissime foliosis, foliis basi dilatatis raptim breviter acuminatis, apice squarrosis.

\section{Gen. Trachypus R. et Hsch.}

T. L.eTUS (R. C.).

Madagascar: province de Betafo, Ambohitantely, igor (Hb. Paris).

T. Appendiculatus (Ren. Card.).

Madagascar: Marohantsetra, baie d'Antongil, Mathiev (Hb. Cardot). 
Gen. Trachypodiopsis Fleisch.

T. SeRrulata (Brid.).

Grande Comore: pente du Kortala, MACÉ, I 900 (Hb. de Poli). Madagascar: Betsileo, Rev. Villaume, 1905 (Hb. Lacouture).

\section{Gen. Orthostichopsis Broth.}

O. subimbricata (Hpe) Broth.

Madagascar: Ivohibe, Igor, Pelletier; Moramanga, Igo I; province d'Antsirabe, Galinon, 1903, (Hb. Paris).

O. Longinervis (Ren. Card.) Broth.

Madagascar: forêt de Ambohimitombo, Dr Forsyth MAJOR, 1895, (Hb. Levier); forêt de Fito, Perrot i 897 ; Ivohibe, Pelletier, 190r, (Hb. Paris); Betsileo, Rev. Villaume, I904, (Hb. Lacouture).

\section{Gen. Pilotrichella C. Müll.}

P. pseudo imbricata C. Müll.

Madagascar: Ikongo, $\mathrm{D}^{\mathrm{r}}$ Besson 1890 ; forêts du versant oriental, Girod Genet, 1897 .

Cette espèce des Comores est nouvelle pour Madagascar.

\section{Gen. Aerobryopsis Fleisch.}

\section{A. pSeudo Capensis C. Müll.}

Les localités de Madagascar citées pour cette espèce dans le Prodrome restent douteuses et sont à supprimer; elles se rapportent à l'A. subpiligera (Hpe) qui a les feuilles plus petites. Toutefois les deux espèces sont extrêmement voisines et parfois difficiles à distinguer. On rencontre fréquemment à La Réunion une forme de $\boldsymbol{A}$. pseudocapensis $\mathbf{C}$. M, dont le port est plus robuste, non plumeux, les feuilles beaucoup plus grandes et plus étalées que dans le type (V. majus R. C.). 
A. subpiligera (Hpe).

Madagascar : province d'Antseranandrotsy, Igor ; province de Betafo, Antsirabe (Hb. Paris).

Gen. Aerobryidium Fleisch.

A. Lanosulum (C. Müll.).

Grande Comore: pentes du Kortala, MACÉ, 1900, (Hb. de Poli).

\section{Gen. Phyllogonium Brid.}

P. viscosum P. Beauv.

Madagascar: forêt de Ambohimitombo, Dr Forsyth Major, Hb. Levier), I894; Betsileo, Rev. Vuillaume, ig04. (Hb. Lacouture). Nouveau pour Madagascar.

Gen. Neckera Hedw.

N. MADAgassa Besch.

Madagascar: Ampasiamadinika (alt. 800 mètres), Charbotel, ig04, (Hb. Paris).

N. Borgenı Kiaer.

Madagascar: province de Betafo, Antsirabe, Igor (Hb. Paris); Betsileo, I 9o5, Rev. Vilinume, (Hb. Lacouture) ; Ambohibeloma, Rev. Camboué, 1906.

Gen. Orthorrhynchidium Ren. et Card. gen. nov.

Caulis primarius repens, radiculosus, corticibus arcte adfixus, foliis minimis, remotis, squamæformibus, concavis, triangulari-lanceolatis, cuspidatis enerviis præditus. Caulis secundarius simplex, usque ad basin foliosus, frondem angustam, linearem, percomplanatam nititam 
sistens. Folia 6-8 sticha sed valde complanata et inde pseudo-disticha, conferta, rigida, omnia naviculari-concava, lateralia conduplicata et equitantia, anguste oblonga, sublingulata, apice, breviter acuminata, recurvula, marginibus planis integris, costa tenui ad 2/3-3/4 producta, rete pallido, cellulis anguste linearibus subflexuosis, lævibus, achlorophyllosis, parietibus angustis, nec sinuosis, nec porosis, alaribus paucis brevioribus parum distinctis. Cætera ignota.

Ce genre diffère des Calyptothecium par les tiges secondaires courtes, simples, completement feuillées et par le tissu non poreux. L'aspect général rappelle complètement celui des Orthorrhynchium; mais les feuilles sont en 6 ou 8 rangées et non vraiment distiques, leur sommet est acuminé et non obtus.

O. planifrons Ren. Card. (Garovaglia planifrons Ren. Par. in Rev. bryol.). (Pl. xvı). - Pallide vel læte viride, nitidum. Caulis repens denudatus corticibus adfixus; caulis secundarius erectus simplex I-2 I/2 cent. altus. Folia frondem angustam linearem, complanatam sistentia, subdisticha, conferta, rigida, erecto-patula, conduplicato-navicularia subasymmetrica, anguste oblonga sublingulata, apice interdum subcucullata (apiculo extus recurvato), I-I I/2 mil. longa, integra vel vix sinuolata ; costa pertenuis ad $3 / 4$ producta ; rete pallido delicatulo denso, cellulis linearibus interdum subflexuosis, apice acutis, lævibus, achlorophyllosis, parietibus angustis nec sinuosis nec porosis, alaribus rufescentibus amplioribus breviter rectangulis, apicalibus brevioribus, subporosis. Cætera desunt.

Madagascar : province de Betafo, Antoagazi (Hb. Paris).

Par le port, cette espèce rappelle l'Orthorrhynchium Nietneri C.M. de Ceylon (Phyllogonium fulgens Mitt. Fl, Ind. Or.); mais les feuilles ne sont pas exactement distiques et possèdent une nervure qui dépasse le milieu. Par ses caractères intimes elle se rapproche de C. Bescherellei (Pilotrichella) Kiaer, et en diffère par la foliation tout à fait aplanie comme dans les Phyllogonium,par les tiges secondaires simples, les feuilles subdistiques plus étroites et plus longues, par la nervure un peu plưs longue, enfin parle tissu serré composé de cellules linéaires à parois minces, ni sinueuses, ni poreuses, non reliées entre elles par des traverses latérales.

Peu de temps après sa découverte à Madagascar, cette espèce a été retrouvée au Congo belge, sous une forme identique.

Gen. Porotrichum Brid.

P. mucronulatulum C. Müll.

Madagascar: Betsileo, Villaume, 1906 (Hb. Lacouture). 
Cette espèce a quelque analogie avec les Orthostichopsis longinervis R. C. O. scaberula R. C. et $O$. debilinervis R. C. (Porotrichum palmetorum Besch) quil affectent souvent dans leur tacies la forme porotrichoïde. En ce qui concerne le P. Robillardi C. M. des Mascareignes auquel le $P$. mucronulatulun $C$. M. est comparé dans le Prodrome, il est difficile d'y voir autre chose qu'une forme grèle de P. Comorense C. M.

\section{Gen. Pinnatella (C. M.) Fleisch.}

\section{P. Geheebil (C. Múll.) var. regulare Ren. Par.}

Tige secondaire haute de $2-3$ r/2 cent. Fronde étroite simplement pennée à rameaux raiđes étalés-dressés, régulièrement décroissants de la base au sommèt, la nervure mesure $33 \mu$ à la base; feuilles caulinaires longues de 0,8 à 0,9 mil.

Le type des Comores, d'après un échantillon original reçu de $\mathbf{M}$. Geheeb, a une taille plus réduite, des divisions courtes, simplement pennées, à rameaux très courts, étalés à feuilles petites.

Dans sa monographie des Porotrichum, le Dr N. C. Kíndberg réunit le $P$. Chenagoni C. Müll. au $P$. Geheebii C. M. Sans doute les caractères qui les séparent ne sont pas de premier ordre; cependant la fronde dilatée bipennée du $P$. Chenagoni C. M. lui donne un port différent qui permet de le distinguer à première vue.

Gen. Campylodontium Doz. Molk.

C. mauritianum C. Müll.

Madagascar: Montagne d'Ambre près Diego Suarez, LeLoUTRE, I 899, Hb. Corbière).

Gen. Entodon C. Müll.

E. Rutenbergil C. Müll.

Madagascar : province de Betafo, Antsirabe, Igor (Hb. Paris).

E. Felicis Ren. Card., var. julaceus Ren. Card.-A forma genuina differt ramis exacte julaceis et foliis cucullatis, haud complanatis.

Madagascar: Ambohimatsara près Ambositra, 1892, Rev. BERTHIEU.

E. Corbieri Ren. Card. - Monoicus, læte viridis, nitidus. Caulis 
$2-3$ cent. longus, irregulariter pinnatus, ramis 4-6 mil. longis, compressis. Folia complanata ovato-acuminata, acumine obliquo, 1,25-1,5o mil. longa, concava, e medio tenuissime et remote, superne distincte serrulata, ecostata; cellulis linearibus, alaribus quadratis. Folia perichætialia vaginantia, ovato-lanceolata, longe cuspidata, 2 mil. longa. Capsula in pedicello pallido, r,5o-1,75 cent. longo, erecta symmetrica vel leniter incurvata, ovato-cylindrica. 3 mil. longa, sicca plicatula, ore angustato interdum obliquo. Peristomii dentes externi o,3 mil. longi, obsolete trabeculati, in dimidio superiore pluries pertusi, granulosi et longitudinaliter striatuli, interni paulo breviores, fissiles. Sporæ parva, laves, I 0-1 $3 \mu$ crassæ.

Madagascar: Montagne d'Ambre près Diego Suarez, Leloutre.

Cette plante qui nous a été amicalement communiquée par $\mathbf{M}$. Corbière à qui nous sommes heureux de la dédier ressemble parleport aux $E$. madagassus $C$. Müll. et $E$. Rutenbergii C. Müll. Le premier, dont nous avons reçu par le Dr Kiær un échantillon stérile récolté dans les Monts Ankaratra par Borgen et vraisemblablement déterminé ou vérifié par C. Müller, n'est connu qu'à l'état stérile. La courte diagnose de cette espèce qui figure dans les Reliquice Rutenbergiana, indique des feuilles étroites lancéolées, subdenticulées au sommet. En l'absence de tout renseignement sur le péristome, il est difficile de se former une idée bien nette de cette plante, qui paraît différer bien peu de E. Rutenbergii C. M.

Ce dernier est mentionné dans le même opuscule par la seule diagnose suivante: "A priori ( $E$. madagassus) differt foliis serrulatis ». Nous tenons encore de feu le Dr Kiær un échantillon fertile de $E$. Rutenbergii collecté par Borgen dans les Monts Ankaratra. Les teuilles sont plus petites et plus étroites que dans $E$. Corbieri R. C.; mais surtout le péristome offre de notables différences. Les dents externes sont géminées, élargies à la base, non percées de trous et divisées dans le haut en deux branches subulées. Dans la moitié supérieure, elles sont marquées de stries obliques et dans la moitié inférieure, de stries transversales. Les processus sont entiers, sans ligne divisurale ; enfin les spores sont rugueuses et beaucoup plus grosses, mesurant $18-20 \mu$.

\section{Gen. Erythrodontium Hpe.}

E. Lacouturei Ren. Card. (Pl. xvir). - Monoïcum. Robustum, cæspites densi, bicolores, nunc smaragdino-virides, nunc rufo variegati, vel omnino fere vinoso-rufescentes, nitidi. Caulis repens radiculosus, flexuosus dense ramosus, rami julacei, rigidi, apice obtusi, erecti, 5-6 mil. longi. Folia densissime conferta, sicca arcte imbricata, humida turgide subimbricata, concava, breviter decurrentia, rotundata vel deltoideo- 
rotundata, I-1,2 mil. longa, o,8-o, 9 mil. lata in apiculum latum brevissimum erectum, haud recurvum coarctata subtiliter denticulata, enervia, plicis brevibus curvatis prædita. Cellulis mediis angustis, elongate rhombeis, $18-20 \mu$ longis alaribus mediocriter numerosis majusculis quadratis, interdum oblatis, sat regularibus, recte seriatis. Folia perichætialia pallida vel plus minus rufescentes, intima perlonga in cuspidem longam subintegram producta. Capsula in pedicello flexuoso, circa I5 mil. longo, crasso pallide rufescente complanato subcanaliculato erecta, oblongo cylindrica, 2-2 I/2 mil. longa, sicca irregulariter plicata, pallide rufescens. Peristomium deest.

Madagascar : Fianarantsoa, Rev. Villaume, i 9o5, (Hb. Lacouture).

Des deux espèces du continent africain auxquelles on peut comparer cette espèce Io subjulaceus C. Müll, en differe à première vue par un port plus grèle, ses rameaux plus longs, incurvés, par les cellules moyennes plus courtes; $2^{\circ}$ E. Engleri Broth. dont la taille est à peu près aussi robuste, s'en distingue par sa couleur entièrement verte, ses feuilles moins densément imbriquées, moins grandes et surtout moins larges, par les cellules alaires plus nombreuses, remontant plus haut le long des marges, plus petites, moins régulièrement sériées, enfin par la capsule un peu plus allongée. Le genre Erythrodontium est nouveau pour Madagascar.

\section{Gen. Trachyphyllum Gepp.}

T. Ferriezir (Besch.) var. abbreviatum Ren. Card. - A planta normali differt, cæspitibus densioribus intertextis, ramis brevibus, obtusis, crassiusculis, arcuatis, foliis minoribus breviter acuminatis.

Madagascar : paraît répandu sur le versant du Mozambique où cette forme croît parfois sur la terre tandis que le type est toujours corticicole.

Le T. Ferrięii ne peut guère être considéré que comme une sous-espèce du T. fabronoides C. M. Cette dernière espèce varie peu dans les Mascareignes; ses rameaux sont plus longs, plus effilés, tandis que c'est la race du $T$. Ferriezii qui domine à Madagascar surtout sur le plateau central et le versant du Mozambique où par suite de périodes de sécheresse, elle devient polymorphe et se présente souvent sous des formes trapues dont le port est bien différent de celui du type. Cependant malgré la prédominance du T. Ferriezii, quelques formes du plateau centrál et de la zone supérieure des forêts m'ont paru bien peu distinctes du T. fabronioïdes C. $\mathbf{M}$. type. 
Gen. Stereophyllum Mitt.

S. immobtoides Ren. Card.

Madagascar: province de Fianarantsoa, Saovano, Croll, I900, (Hb. Paris).

Nouveau pour Madagascar.

Gen. Fabronia Raddi.

ANALYSE DES ESPÈCES

1. Feuilles linéaires très longuement ciliées dès la base, cettules linéaires, les alaires non différenciées. Péristome nul............

Feuilles ovales ou lancéolées, cellules rhombées, les alaires différenciées. Péristome simple ..................... 2

2. Feuilles ovales, obtuses, entières..........F. Motelayi Ren. Card.

Feuilles aiguës cuspidées............. 3 .

3. Feuilles ciliées................... 4.

Feuilles subentières ou dentées, non ciliées.. 5.

4. Feuilles lancéolées ou oblongues-lancéolées insensiblement rétrécies.............. F. Campenoni Ren. Card.

Feuilles ovales contractées en une cupide

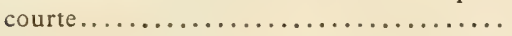

5. Feuilles grandes (long. 0,75-1 mil., larg. 0,25 mil.) oblongues ou ovales à nervure longue et assez forte dépassant le milieu..........

Feuilles plus petites à nervure plus faible atteignant à peine le milieu ...............

6. Feuilles insensiblement rétrécies, tissu épaissi

Feuilles contractées en une cuspide courte, tissu non épaissi....................

7. Feuilles insensiblement rétrécies acuminées

cuspidées..................... 8.

Feuilles contractées en une subule fine plus ou

F. Persooni Schwgr.

F. Villaumii Ren. Card.
7.

F. crassiretis Ren. Card.

F. Guienzii Hpe. 
moins longue.................... 9.

8. Feuilles lancéolées ou oblongues-lancéolées longuement et finement cuspidées, dentées assez fortement dans les $2 / 3$ inférieurs, presque entières dans le $1 / 3$ supérieur.Capsule relativement grande, oblongue...... Feuilles oblongues ou ovales-oblongues brièvement acuminées, faiblement denticulées.

9. Feuilles oblongues lancéolées fortement dentées assez longuement cuspidées. Capsule petite subglobuleuse................. Feuilles ovales ou oblongues contractées en une cuspide courte, faiblement denticulées

F. Vallis gratia Hpe.

F. fastigiata Ren. Card.

F. Garnieri Ren. Par.

F. Lachenaudi Ren.

F. Villaumii Ren. Card. (Pl. xvin.) - Monoica. Minutula. Caulis repens, rami brevissimi I mil. longi, crassiusculi, apice obtusi. Folia densissime conferta, imbricata late ovata, o, 55 mil. longa, 0,25 mil. lata, subito in cuspidem longiusculam coarctata; marginibus ciliatis ; costa basi crassiuscula ad medium producta; cellulis mediis $40-43 \mu$. longis. Folia perichetialia brevia 0,4 mil. longa, ovata, alis inflexis concava, ex apice valde eroso-dentato, subito in apiculum producta. Capsula in pedicello curvato 3 mil. longo ovata, collo distincto siccitate rugoso instructa, ore aperto incrassato. Peristomii dentes I9 mil. longi late lanceolati, obtusi medio inferiore transverse striolati, superne longitudinaliter punctulati. Membrana exothecii e cellulis rectangulis subregularibus parietibus parum undulatis composita. Sporæ valde muricatæ inæquales $16-23 \mu$ crassæ.

Madagascar: Fianarantsoa, ad terram saxosam cryptarum, Rev. Villlaume igo4 (Hb. Lacouture).

Cette espèce se distingue facilement du $F$. Campenoni Ren. Card. qui a comme elle les marges ciliées, par les feuilles ovales contractées en une cuspide médiocre et par la nervure plus.épaisse à la base. Le $F$. Campenoni a en outre les rameaux plus allongés, plus grèles, flexueux d'un aspect soyeux et la capsule subglobuleuse. (Pl. xviri).

F. Garnieri Ren. Par. (Pl. xrx), - Monoica. Minuta. Cæspites depauperati ad cortices duras sparse crescentes. Rami graciles perbreves sericei. Folia brevia 0,45 mil. longa, oblonga vel oblongo-lanceolata sat rapide in cupidem longiusculam angustata, marginibus regulariter serratis, haud ciliatis ; costa tenella ad medium vel sæpius infra disso- 
luta. Cellulis mediis rhombeis $56-59 \mu$ longis. Folia perichætialia oblonga breviter cuspidata apice denticulata, 0,65 mil. longa. Capsula in pedicello curvato $\mathrm{I} \mathrm{I} / 2 \mathrm{mil}$. longo, late ovata subglobosa. Peristomii dentes o, 2 mil.longi obtusissimi, transverse striati superne irregulariter punctulati. Membrana exothecii e cellulis sat regulariter rectangulis parietibus minute undulatis composita. Sporæ granulosæ inæquales 16-19 9 crassæ.

Madagascar: province de Betafo, rive droite de la Mania (Hb. Paris).

Cette espèce très voisine de $F$. Vallis-gratia Hpe du Cap. en diffère par ses feuilles plus courtes, plus rapidement rétrécies en une subule moins allongée,par les marges plus régulièrement dentées dans le tiers supérieur, enfin par la capsule plus petite subglobuleuse. Dans le $F$. Vallis gratia Hpe, les feuilles sont plus étroites, lancéolées et longues de $0,75 \mathrm{mil}$; en outre les spores paraissent un peu plus petites, ne dépassant pas $16 \mu$. Il est possible toutefois que cette plante ne soit qu'une race régionale ou sous-espèce de $F$. Vallis gratia Hpe. (Pl. xvini).

F. Lachenaudi Ren. (Pl. xvir). - Monoïca. Pallide viridis cæspites laxiusculi sat extensi. Caulis radiculosus repens, rami flexuosi vel curvuli. Folia sat dense conferta apice subsecunda e basi ovata brevi sat subito breviuscule tenuiter cuspidata, I/2 mil. longa, minute irregulariter denticulata, costa medium versus evanida; rete pallide luteo sat pellucido, utriculi primordialis vestigio conspicuo, cellulis alaribus quadratis, cæteris elongate rhombeis mediocriter amplis. Folia perig. concave apice grosse eroso-dentata. Antheridia 3-4. Folia perich. minuta, semi-vaginantia ovato-cuspidata, denticulata. Capsula in pedicello pallido 3 I/2 mil. longo ovato-globosa, ore dilatata. Membrana exothecii e cellulis iregularibus valde sinuoso-undulatis composita. Sporæ majusculæ 19-23 $\mu$ crassæ. Peristomium deest.

Madagascar: province d'Ankazobé, district de Fihaounana (Hb. Paris).

Cette espèce, qui se rapproche par le port des $F$. Vallis Gratice Hpe du Cap et F. Campenoni R. C. de Madagascar, s'en distingue nettement par les feuilles courtes, plus larges, ovales à la base,rapidement contractées en une cuspide fine et médiocre, faiblement denticulées, nullement ciliées ou dentées; elle differe des $F$. fastigiata R. C. et $F$. crassiretis $R, C$. par le port et la forme des feuilles. D'autre part le $F$. Guienzii Hpe du Cap a les feuilles du double plus grandes,plus longuement nerviées, les cellules plus amples, etc. 
Gen. Lepidopilum Brid.

L. Corbieri Ren. Card. (Pl. xxi). - Monoïcum, pallide viride, robustum. Cespites laxi, plagiothecioidei. Caulis depressus, $2-4$ cent. longus, simplex, rarius parce ramosus. Folia lateralia remotiuscula, patula, complanata, flexuosa, subrecurvata, basi rotundata, 2 mil. longa, paulisper asymmetrica, ovata vel oblonga sat subito acuminata, acuta, interdum breviter et tenuiter cuspidata, elimbata, e medio denticulata, costis binis subæqualibus paulo ultra medium productis, cellulis elongate subhexagonis, mediis $4^{-6}$ longioribus quam latioribus, utriculo primordiali conspicuo. Folia dorsalia latiora, breviora, late ovata, subito breviter cuspidata, subintegra, costis binis vix ad medium productis. Folia perichætialia multo minora integra, ecostata. Capsula in pedicello brevi, circa 5 mil. longo, pallido, e medio scabro inclinata vel horizontalis, subcylindrica, I, 25 mil. longa, castanea, collo longo defluente instructa, sub ore leniter constricta.Exostomii dentes siccitate intus curvati, o, 4 mil. longi, transverse striati, linea divisurali hic illic late aperta, marginibus sublcevibus, haud cristatis; endostomii processus e membrana usque ad medium versus dentium producta anguste lanceolati, integri, æquilongi, haud rimosi, erecti; cilia singula. Sporæ læves, $25 \mu$ crassæ.

Madagascar : Montagne d'Ambre près Diego Suarez, LELoutre, 1899 (Hb. Corbière).

Voisin parle port du L. Darutyi Besch., de Maurice, mais en diffère par les feuilles plus espacées, aiguës, par le pédicelle plus court, par la capsule longue et étroite, munie d'un long col défluent, et par les dents de l'exostome dépourvues de crêtes marginales.

Le $L$. furcatum Mitt., de Ceylan, a aussi quelque ressemblance avec notre plante; mais les feuilles sont plus étroites, plus longuement cuspidées, le tissu est plus serré, le pédicelle de moitié plus court, la capsule plus renfée, plus courte, longue seulement de $\mathrm{I}$ mil.

On ne peut d'ailleurs confondre le $L$. Corbieri avec les autres espèces des îles austro-africaines, dont le système végétatif paraît déjà, à première vue, moins développé. Il en diffère en outre par les caractères suivants :

10 du $L$. diversifolium Ren. et Card. par sa taille plus grande, la forme et la dentelure des feuilles.

$2^{\circ}$ du L. Isleanum Besch. par les tiges divisées, et par le pédicelle plus long, scabre dans toute la moitié supérieure. 
3o du L. flexuosum Besch. par les feuilles beaucoup plus grandes, le pédicelle plus court, la capsule horizontale et les dents de l'exostome dépourvues de crêtes marginales.

L. subrevolutum Ren. Card(Pl. xx).-Monoicum. Cespites pallidi sat densi. Caulis prostratus brevis, ramis vix 10 mil. longis. Folia sat dense conferta haud curvata sicca flexuosa, lateralia parum asymmetrica, oblonga I I $/ 4 \mathrm{mil}$. longa, I/2 mil. lata, apice sat raptim brevi acuminata, dorsalia angustiora sensim longius angustata, marginibus pro more revolutis superne minute denticulatis vel subintegris. Costis binis ad medium productis. Rete sat denso, cellulis mediis elongate hexagonis utriculi primordialis vestigio plus minus repletis. Folia perichætialia multo minora, hyalina, intima integra longiuscule cuspidata. Capsula in pedicello crasso pallide rubello flexuoso, vix ro mil. longo superne tuberculoso, horizontalis I I/4 mil. longa fusco-purpurea oblonga collo instructa sicca sub ore contracta. Exostomii dentes o,38 mil. dense trabeculati, trabeculis arcuatis prominentibus, marginibus valde cristatis, linea media in $3 / 4$ longitudinis late aperti, processus haud fissi. Membrana exothecii e sellulis majoribus contexta; operculum alte convexum rostratum; Calyptra pallida apice fulva laciniata.

Madagascar: forêt de Analamazaotra, Rev. Cambouḱ, I 890.

I. Chenagoni Ren. Card. - Monoicum. Caulis erectus r, 5 cent. Iongus inæqualiter pinnatus, rami 5 mil. longi patuli. Folia laxiuscule conferta, humida erecto-patentia, parum reflexa, sicca flexuosa, dorsalia elongate lanceolata I I/2-I 3/4 mil. longa sensim longe acuminata, lateralia asymmetrica, interdum apice sat raptim acuminata ; marginibus superne leviter sed acute serratis; costis binis irregularibus ad medium productis, non nunquam vix notatis; cellulis in medio inferiore hyalinis, basilaribus elongatissime subhexagonis ferе linearibus, tenuiter limitatis, mediis elongate rhombeis angustis, superioribus (in foliis lateralibus) brevioribus rhombeis vel subellipticis, crasse et sub apice crassissime limitatis. Pedicellus rubellus tenuis 5-7 mil. longus, flexuosus; capsula vetusta obliterata, parvula pendula. Cætera desunt. 1890.

Madagascar: Montagne d'Ambre près Diego Suarez, Chenagon,

L'exiguité de notre échantillon ne permet pas de donner une description plus complète. Toutefois cette espèce par la forme de ses feuilles et. leur texture (cellules petites, les inférieures étroites et allongées) ne peut être confondue avec 
aucune autre de notre Domaine. Elle ne pourrait guère être comparée qu'au L. Humbloti Ren. Card. de la Grande Comore qui en diffère d'ailleurs essentiellement par ses feuilles beaucoup plus finement acuminées, subulées, entières et énerves.

Gen. Gyclodictyon Mitt.

C. Auberti Pal. Beauv. - Dentes exostomii o,40-0,42 mill. longi; linea divisurali usque ad 3/4 longitudinis late aperti, dense trabeculati, trabeculis valde prominentibus (marginibus alte cristatis) apice decolorato papillis acutis hirsuto. Processus regulariter inter articulationes

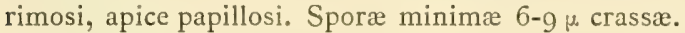

Madagascar: Beteileo, Villaume igo4; Ambohibeloma, Rev. CamBouÉ, I 9o6, (Hb. Lacouture).

Cette espèce doni je n'avais reçu que quelques maigres échantillons du littoral oriental, paraît assez répandue dans les localités précitées où elle se développe sur la terre en larges touffes fertiles. Aucune autre espèce de Cyclodictyon n'a encore été constatée à Madagascar.

\section{Gen. Gallicostella C. Müll.}

\section{Perroti (Ren. Card.) Paris. (Callicostella heterophylla Ren.Card.)}

Le nom de $C$. heterophylla ayant été déjà donné par Aongström à une espèce de l'ile S. José, un changement de nom s'imposait pour l'espèce de Madagascar.

\section{Gen. Rhacopilum P. Beauv.}

R. MAdagassum Ren.

Madagascar : forêt de Ambohimitombo, Forsyth major, I 894 (Hb. Levier). Ambohibeloma, Rev. Cambouḱ, 1906 (Hb. Lacouture).

Var. grandistipulaceum Thér. - Foliis stipulariis multo majoribus, foliis caulinis fere æmulis, brevius cuspidatis.

Madagascar: Betsileo, Rev. Villaume (Hb. Lacouture). 
R. Plicatum Ren. Card. Poli).

Grande Comore : pentes du volcan Kortala, MAcÉ, I9oo, (Hb. de

M. Thériot a reconnu qu'une plante de l'Usambara distribuée sous le nom de $R$. capense C. M. avant la fondation du $R$. plicatum R. C. appartenait à cette dernière espèce. Or comme j'avais étudié le R. capense C. M. d'après l'échantillon de l'Usambara, il s'en suit que la note descriptive publiée dans le Prodrome (page 269) ainsi que les figures relatives à cette espèce dans l'Atlas des Mousses de Madagascar doivent être annulées. Le véritable $R$. capense C. M. que j'ai pu étudier depuis sur des échantillons du Cap et du Natal diffère essentiellement du $R$. plicatum R. C. par ses stipules élargies et cordiformes à la base.

Gen. Pseudoleskea Br. et Schimp.

P. obtusiuscula Ren. Card. (Pl. xxII). - Pallide viridis. Cæspites humiles, depressi, densiusculi. Caulis repens passim radiculosus, inortinate divisus, rami, graciles, julacei, incurvati. Folia humida erectopatentia, sicca arcte appressa o,6 mil. longa, ovata brevissime et obtiuscule acuminata, basi breviter decurrentia, marginibus planis vel infima basi revolutis, superne minutissime denticulatis vel basi subintegris; costa paulo ultra medium producta.Cellulis elongate subhexagonis duplo longioribus quam lat., utriculi primordialis vestigio flexuoso repletis, alaribus oblate quadratis, omnibus fere lævibus, parietibus haud incrassatis. Folia perichætialia erecta oblonga acuminata, intima I mil. longa, ecostata, apice remote denticulata vel integra pallide et elongate texta. Capsula in pedicello brevi, 2 I/2 mil. alto erecta, minuta, I mil. longa. Operculum convexum sat longe curvirostratum.

Madagascar: Fianarantsoa, ad truncos, Rev. Villaume, 1904, (Hb. Lacouture).

Cette espèce que l'on peut comparer au Ps. claviramea C. Müll. du Cap s'en distingue immédiatement par un port plus grêle, par ses rameaux filiformes non épaissis au sommet, par ses feuilles plus petites non cordées à la base à nervure dépassant peu le milieu, enfin par ses cellules nettement anguleuses, subhexagones à parois non épaissies. Le pédicelle est aussi plus court et la capsule plus petite. 


\section{Gen. Thuidium Schimp.}

T. subscissun C. Müll.

Madagascar: Maintirano sur la côte du Mozambique (Hb. Paris).

T. Chenagonı C. Müll.

Madagascar: pays des Baras, cercle d'Ivondro, Saorano, rgoo, (Hb. Paris) ; province d'Ambosika, vallée : pleurs des Boufs, Savelli, 1904, (Hb. Paris).

Cette espèce de Diego Suarez est comparée dans le Prodrome au T. subscissum C. M. dont elle est distincte; elle paraît surtout voisine de T. perscissum C. M. d'Anjouan et de Mayotte.

T. trachynoton Ren. Par. - Cæspites laxi lutescentes inter alios muscos vigentes. Caulis $2-4$ cent. longus, flexuosus simpliciter et irregulariter pinnatus, paraphyllis densis brevibus. Folia caulina erecta vel apice subsecunda, e basi late ovata cordata raptim longiuscule acuminata (long. 3/4-1 mil.), plicatula, marginibus eroso papillosis vel denticulatis recurvis. Costa valida dorso spinulosa usque apicem versus fere continua. Folia ramea incurvata ovato-vel oblongo-acuminata denticulata, I/2 mil.longa, evanidinervia, cellula apicalis emarginata bipapillosa, omnia pallide et distinctissime reticulata, cellulis oblongo-ellipticis papilla unica magna dorso valde et acute prominente ornatis. Folia perichæetii (haud evoluti) externa breviora squarrulosa, intima erecta oblongo-lanceolata in acumen loriformem producta, I I $/ 4 \mathrm{mil}$. longa, usque ad apicem fere tenuiter costata, marginibus passim revolutis plus minus distincte crenulatis vel remote denticulatis, non ciliatis, lævibus haud plicatis. Capsula immatura I I/2 mil. longa, operculum rugulosum. Pedicellus pallide purpureus i $1 / 2$ cent. longus, lævis. Cætera inquirenda.

Madagascar : province de Betafo, rive droite de la Mania, Galinon, I 90 I (Hb. Paris).

Cette espèce se distingue à première vue du Th. yersicolor Hrsch. du Cap par un port plus robuste, İles touffes très lâches, les tiges beaucoup plus longues, simplement pennées, pas les feuilles caulinaires et raméales du double plus grandes, plus 
longuement acuminées. Elle a aussi des rapports avec le Th.borbonicum Besch. dont elle diffère par le port tout autre, lâche, plus robuste, les tiges plus longues simplement pennées, les feuilles caulinaires plus grandes dressées appliquées, par le tissu plus transparent, par la saillie plus forte et aculéiforme des papilles dorsales, par le pédicelle un peu plus court. Elle ressemble par le port au Th. tenuisetum R. C. du Congo, mais ce dernier a les feuilles crispées, plus brièvement acuminées, les papilles dorsales peu saillantes, les folioles périchétiales 2 fois plus longues, ciliées.

\section{Gen. Brachythecium Sch.}

B. ATRotheca Duby.

Madagascar: province d'Andevorante, bords de l'Iaroka à Marovato, Igo I, (Hb. Paris); Betsileo, Rev. Villaume; Ambohibeloma, Rev. Cambouk, igo6 (Hb. Lacouture).

Cette espèce est surtout répandue dans la zone supérieure des forêts où les tiges s'allongent, s'étalent et deviennent assez régulièrement pennées. Sur les argiles rouges du plateau central croît une forme plus trapue à ramification courte à touffes encombrées de terre à la base.

B. Borgeni Hpe.

Madagascar : Betsileo, Rev. Villaume, 1906, (Hb. Lacouture).

Cette espèce me semble bien difficile à distinguer à l'état stérile du $B$. atrotheca Duby. A l'état fertile, jerapporte au $B$. Borgeni Hpe les formes d'une taille plus réduite à touffes assez denses, ne s'allongeant pas en tiges étalées et pennées, à capsule courte dressée ou presque dressée, à peine incurvée, ne noircissant pas avec l'âge. Dans l'espèce voisine la capsule est allongée, parfois horizontale, fortement incurvée et à la fin noirâtre.

B. Mittenii E. G. Britton et Ren. - Caulis circa 4 cent. longus apice flagellato-attenuatus, sat dense et inordinate pennatus. Folia nitida dense conferta erecto-patentia, rigidiuscula, caulina I I/2 mil. longa, e basi late ovata deltoidea longiuscule acuminata, cuspidata, ramea angustiora, oblongo-lanceolata, biplicata, marginibus planis, toto ambitu dense et minute denticulata. Costa usque ad $2 / 3$ vel $3 / 4$ producta. Rete denso hypnoideo, cellulis, linearibus 8-12 longioribus quam lat. vermicularibus sæpe flexuosis apicibus pro more obtusiusculis et tenuiter prominulis, basilaribus laxioribus, elongate-subbexagonis, alaribus numerosis, granulosis quadratis vel breviter rectangulis. Cætera desunt.

Madagascar: sine loco nec nomine in $\mathrm{Hb}$. Mitten. 
Cette espèce qui m'a été gracieusement communiquée, par Mrs. E. G. Britton, du Botanical Museum de New-York qui possède l'herbier de Mitten, diffère essentiellement des B. Borgeni Hpe et B. atrothecaDuby de Madagascar et du B. implicatum Mitt. du Natal par son tissu foliaire beaucoup plus dense, vermiculaire. Dans ce dernier, les feuilles sont d'ailleurs entières. A première vue le $B$. Mittenii se distingue de ces espèces voisines par ses feuilles raméales moins serrées, plus raides et plus étalées, fortement plissées à l'état sec. En l'absence de fructifications, il est difficile de décider si cette espéce est un Brachythecium ou un Eurhynchium.

\section{Gen. Sematophyllum Mitt.}

S. Stellatum Ren. Card.

:Madagascar : province d'Andevorante, forêt de Fito, PErrot, (Hb. Levier).

\section{Gen. Acanthocladium.}

A. Flexile (Ren. Card.) (Sematophyllum Subg. Trismegistia R. C. in Prod. Madagas.)

Madagascar : Anosibe, Verdolin, I 90 r, (Hb. Paris) ; Betsileo, Rev. Villaume, I 905 ; Imano. Rev. Camboué, 19o6, (Hb. Lacouture).

Tous ces échantillons appartiennent à la forme contractée à laquelle il est fait allusion dans le Prodrome (page 235) et qui, à en juger par ces nouveaux apports, représente plutôt le type, tandis que la description princeps du Prodrome s'applique à la forme émettant des divisions allongées flexueuses à feuilles peu serrées plus étalées. Dans le type, les touffes sont plus denses, les tiges entrelacées, les rameaux courts à feuilles plus serrées subimbriquées s'enroulant parfois un peu en pointe à l'extrémité.

L'espèce se distingue d'ailleurs de ses congénères de notre Domaine par les cellules alaires non dilatées, petites, carrées, généralement remplies de granulations. Malgré cette particularité qu'elle possede en commun avec A.Trichocoleum C.M. du Kilimandjaro, elle n'est pas moins bien à sa place dans ce genre qui a été formé du Subgenus Trismegistia du genre Sematophyllum Mitt. et qui comprend dans notre Domaine, outre A. flexile R. C., A. Rutenbergii (C. M.), R. C. A.protensum Ren. Card. A. Bessoni R. C. 


\section{Gen. Rhaphidostegium Schimp.}

R. Duisaboanum Besch. var. argillicola Ren. Card. - A planta normali differt cæspitibus densioribus, ramis brevibus, erectis, foliis haud subsecundis, multo minoribus, cellulis mediis brevioribus, alaribus minus evolutis, capsula plerumque subglobosa. Forsan species propria.

Madagascar: Tananarive sur la terre argileuse, DoRR, 1897.

R. Sinuosulum Besch.

Madagascar: Ambositra, Rev. Villaune, 1906 ; Ambohibeloma, Rev. Camboué, igo6, ( $\mathrm{Hb}$. Lacouture).

Cette espèce de La Réunion est nouvelle pour Madagascar.

\section{Gen. Trichosteleum Mitt.}

T. læviusculum Ren. Card. - A T. Perroti Ren. et Card. differt: foliis breviter acuminatis concavis, marginibus inflexis, papillis dorso vix prominentibus sparsis, interdum obsoletis, reteque pallidissimo, cellulis angustioribus, longioribus. Pedicellus superne et capsula in dimidio inferiore tuberculosi. Peristomium et sporæe ut in T. Perroti.

Madagascar: forêt de Fito, district de Tamatave, Perrot, 1894, Hb. Levier).

T. Microdontrum Besch. var. megapterum Ren. Card. - A planta typica borbonica differt : foliis longius cuspidatis, apice flexuosis, cellulisque alaribus 3-5, maximis, longissimis. Capsula oblonga. (Atlas des Mousses de Madagascar, pl. io3, fig. 2).

Madagascar: forêt d'Analamazaotra, Rev. Cambouté et Campenon).

T. Perroti Ren. Card. var, eurydictyon Ren. et Card. - A forma normali differt rete laxiore, e cellulis multo latioribus hexagono-subrhombeis composito. (Atlas des Mousses de Madagascar, pl. 104, fig. 2).

Madagascar: île Sainte-Marie, Charly Darbould, I 893. 
T. Leptorhynchum (Brid.) Ren. Card. var. madagassum Ren. Card. - A planta typica borbonica differt: habitu paulo humiliore, foliis minoribus, falciformibus, valde curvatis, dorso evidentius papillosis, capsula breviore, torulosa, exostomii dentibus brevioribus, denique sporis minoribus ( 1 I $-12 \mu$, loco $22-23 \mu$ ). (Atlas des Mousses de Madagascar, pl. 106, fig. 2).

Madagascar : forêt d'Analamazaotra, Rev. Camboué et Campenon, I 890.

Cette forme est bien distincte du type de La Réunion. Les dents de l'exostome n'atteignent guère en longueur que o,29 mil. au lieu de 0,47 dans le type. Les parois des cellules de l'exothèque sont très épaissies et présentent des renfiements noduleux qui les rendent irrégulières, tandis que dans le type, ces renflements sont nuls ou à peine marqués. Sous divers rapports, cette forme se rapproche beaucoup du Raphidotesgium Pervillei (Sch.) Besch, et n'en differe que par les papilles dorsales des feuilles distinctement saillantes, par les folioles périchétiales et le pédicelle plus allongés. Il faut remarquer que dans le $T$. leptorhynchum de La Réunion les papilles dorsales sont faiblement indiquées et parfois à peine visibles, de sorte que, sans un examen attentif, on risquerait de prendre cette espèce pour un Raphidostegium.

T. BoRbonicum (Bel.) var. brachycarpum Ren. Card. - A planta typica borbonica differt: cespitibus intense viridibus, foliis brevioribus, magis hamatis capsulaque breviore. (Atlas des Mousses de Madagascar, pl. I07, fig. I).

Madagascar: Diego Suarez, Chenagon, 1890.

C'est aussi à cette forme qu'ıl faut rapporter le Sigmatella (Thelidium) tamata. vensis C. Müll. in herb. Levier, collecté à Fénérive par Perrot en 1896.

\section{Gen. Taxithelium Spruce.}

T. (Oligostigma) serratum Ren. Card. - Laxe cæspitosum, pallide vel lutescenti-viride. Caulis repens, corticibus radiculis glomeratis adfixus, flexuosus, 5-8 cent. longus, sat regulariter ramosus, ramis compressiusculis, 5-8 mil. longis. Folia erecto-patentia, subcomplanata, concava, enervia, I mil. longa, ovato-acuminata, marginibus jam e basi apicem versus grossius serratis; cellulis oblongo-rhombeis, medio papilla unica maxima ornatis, papillis dorso valde cylindraceo-prominentibus, cellulis alaribus $2-3$ vesiculosis, luteis. Capsula in pedicello lavi, purpureo, tenui, circa $25 \mathrm{mil}$. longo, horizontalis, arcuata, elongate oblonga, 1,50 mil. longa, sicca sub ore leniter constricta,operculo alte conico. 
Madagascar : forêts aux environs de Mahambo, Perrot I 890 ; sine loco (Hb. Rev. Friren); île Sainte-Marie, Perrot 1897; Fenerive, PERrot 1896. (Hb. Levier sub. nom Sigmatella (Papillidium) taxicaulioides C. M. in litt. ad Cl. Levier).

Comores: Anjoulan, Lavanchie (Hb. Muséum Paris).

Espèce bien distincte par les feuilles fortement dentées en scie, les cellules unipapilleuses, à papilles fortement saillantes sur la face dorsale et les cellules alaires vésiculeuses, colorées, comme dans les Trichosteleum. Dans les Taxithelium de la région connus jusqu'à ce jour, les cellules sont munies de papilles très petites, disposées en série et les cellules des angles ne sont pas vésiculeuses. Le Taxithelitum hirtellum Par. Ren. seul a les papilles un peu grosses et vaguement sériées, de sorte que le $T$. serratum Ren. Card. occupe une place toute spéciale dans le genre Taxithelium, constituant un type intermédiaire entre ce dernier genre et les Trichosteleum, mais ne pouvant pas être réuni à celui-ci à cause de l'opercule nettement conique, nullement rostré.

T. hirtellum Par. Ren. - Monoïcum, pallide viride. Caulis repens corticibus adfixus $\mathrm{I}-2$ cent. longus sat regulariter pinnatus, rami patuli breves I $\mathrm{i} / 2-2$ mil. longi. Folia suberecta imbricata concava vix complanata ovato-oblonga basi paululum contracta $3 / 4$ mil. longa e medio vel paulo ultra angustata longiuscule acuminata ecostata, marginibus passim involutis, interdum e basi sæpius e medio remote subtiliter eroso-denticulata et inter dentes papillis dentiformibus ornata. Cellulis alaribus plus minus numerosis (6-14) quadratis haud vesiculosis, vacuis, mediis superioribusque elongatis flexuosis lineari-fusiformibus nunc sat regulariter 4-papillosis, nunc præcipue dimidio inferiore folii papillis in parietibus vicinis equitantibus veluti sparsim papillosis, papillis fere omnibus remotiusculis latis dorso valde prominentibus, nunc cylindraceis vel acutis vel pedicellatis apice dilatato bifidis. Folia perichætialia intima oblonga longe attenuata I I/2 mil. longa integra vel summo vix crenulata, lævia (tantum apice obsolete papillosa). Capsula in pedicello 6 mil. longo purpureo arcuato inclinata vel horizontalis minutissima $1 / 2$ mil. longa, demum purpureo-fusca, ovata. Peristomii perbrevis dentes ext. 0,24 mil.longi obsolete cristati, interni e membrana alte producta subæquilongi, carina integri. Cilia singula breviora robusta dense papillosa. Sporæ læves pusillæ 10-I $2 \mu$ crassæ.

Madagascar : Cercle de Maintirano, Igor (Hb. Paris); province de Betafo, rive droite de la Mania, Galinon, 1901 (Hb. Paris).

Cette plante a de l'analogie surtout avec le Raphisdostegium loucoubense Besch. (Fl. de La Réunion, etc.) de Nossi Be et de Sainte-Marie de Madagascar ou du moins 
avec l'échantillon original de M. Bescherelle et qui est certainement un Taxilhelium. Cet échantillon s'accorde du reste parfaitement avec la description sauf cn ce qui concerne la denticulation des feuilles, qui sont très finement denticulées à dents souvent simplement papilliformes plutôt que "argute serrata ". Les angles hasilaires sont à peu près dépourvus de cellules carrées qui se remarquent daus le $T$. hirtellum, en nombre inégal, il est vrai, dans deux échantillons récoltés à Maintirano (6-8 cellules dans l'un et 12-14dans l'autre); en outre, tandis que dans le $T$. loucoubense les papilles sont très denses, petites, disposées en lignes bien régulières, dans notre plante ces papilles sont moins serrées, grosses, dilatées et chevauchent souvent sur les parois des cellules voisines de façon à ne plus former de lignes régulières, et font fortement saillie sur la face dorsale en protubérances cylindriques ou dilatées bifides au sommet. Le péristome est moins élevé et les cils deux fois plus courts. Les spores sont aussi un peu plus petites (ro-12 $\mu$ au lieu de $13-15 \mu)$. La grosseur, la disposition moins régulière et la saillie plus forte des papilles qui font classer le $T$. hirtellum dans le sous-genre Oligostigma R. C. et qui le différencient du T. loucoubense, aident aussi à le séparer de plusieurs espèces très affines de la région dont il est utile de rappeler quelques autres traits distinctifs pour éviter de faciles confusions.

Le T.glaucophyllum Besch. a les feuilles plus larges entières plus brièvement acuminées ou apiculées.

Le T.planulum Besch, a la foliation comprimée et la capsule dressée.

Le T. latum R. C. a les feuilles plus brièvement acuminées, les folioles périchétiales papilleuses à cuspide nettement denticulée et la capsule pendante.

\section{Gen. Microthamnium Mitt.}

M. Lacouturei Ren. Card. - Monoicum. Cæspites sat late et longe extensi. Caulis repens $\mathrm{I}-2$ cent. longus radicans, corticibus adfixus, inordinate ramosus, rami intricati, haud vel vix arcuati. Folia caulina, I mil. longa e deltoidea basi acuminata, breviter cuspidata, subecostata, e medio denticulata; ramea paulo longiora ovata vel oblonga vel oblongolanceolata, 1/2-1/3 mil. lata sat subito brevi acuminata, e medio denticulata, superne subserrulata, sat distincte bicostata, marginibus inferne recurvis. Cellulis elongatis apice papilloso-prominulis, Folia perichætialia I I/2 mil. longa e basi ovata vel subdeltoidea sat longe subulata, ecostata, subintegra. Capsula in pedicello 10-12 mil. longo purpureo, crassiusculo, lævi, inclinata vel suberecta inæqualis, parvula, vix I mil. longa ovata vel oblongo-ovata. Operculum conicum rostellatum.

Madagascar: Betsileo, province d'Ambositra, Rev. Villaume, rgo6, (Hb. Lacouture).

Cette espèce forme, sur l'écorce des arbres, en mélange avec Neckera Borgeni Kiær des tapis denses, aplanis, étendus. Elle rappelle par le port robuste et trapu le 
M. pseudo reptans C. Müll. du Transwaal, mais s'en distingue nettement par les feuilles raméales beaucoup moins fortement dentées, par le pédicelle plus court et la capsule plus petite, parfois subglobuleuse.

Elle ne peut être confondue avec $M$. serratum $\mathrm{P}$. B. de La Réunion à cause de ses rameaux entrelacés, mais non arqués procumbants, de ses feuilles raméales plus grandes, plus larges surtout, beaucoup moins fortement dentées en scie, enfin du pédicelle plus court et de la capsule plus petite.

La plupart de ces caractères la séparent aussi de $M$. madagassum Besch. dont le port est moins robuste. Sous ce dernier nom le Dr Kiær m'a communiqué deux échantillons provenant des Monts Ankaratra (Borgen) et qui ne s'accordent pas entièrement avec le petit specimen original que je tiens de Bescherelle lui-même.

\section{Gen. Isopterygium Mitt.}

I. gracile Ren. Card. - Humile, tenellum, lutescenti-viride. Caulis gracilis, repens, radiculosus, 8-12 mil. longus, sat regulariter pinnatus, ramis plumosis brevissimis, I-2 mil. longis. Folia erectopatentia, flexuosa, anguste lanceolata,-longe et tenuiter acuminata, o,75I mil. longa, 0,17 mil. lata, ramea minora angustissima, enervia, marginibus planis subintegris vel summo remote crenulato-denticulatis, rete denso, pallido, cellulis linearibus vix hexagonis apice acutis, indistincte flexuosis, 10-12 longioribus quam latioribus (long. circa $80 \mu$, lat. $6 \mu$, alaribus paucis, brevioribus quadratis vel breviter rectangulis, parum conspicuis. Cætera desunt. (Allas des Mousses de Madagascar, pl. 108, fig. 4).

Madagascar: région du Nord-Ouest, entre les lobes d'un Aneura (A. palmata), Pervillé (Hb. Bescherelle).

Cette Mousse, qui nous a été communiquée par Bescherelle en 1894 se distingue de ses congénères de la région par son port grêle, ses rameaux plumeux, ses feuilles étroitement lancéolées, flexueuses, longuement et finement acuminées. Ces caractères la séparent notamment de l'I. subleploblastum Besch., qui a aussi un port grêle, mais des feuilles plus larges et moins longuement et finement acuminées. L'I. citrinellum Ren. et Card., avec lequel on pourrait confondre notre plante à cause de l'analogie entre la forme de leurs feuilles, croît en touffes plus denses, ses feuilles sont plus longuement cuspidées, distinctement binerviées, denticulées presque dès la base, les dents devenant très saillantes, aiguës, à la subule:

I. minutum Ren. Card. - Monoicum, e minutissimis, pallide viride, nitidum. Caulis repens radiculosus, 4-6 mil. longus, vage ramosus, ramis brevissimis. Folia erecto-patentia plus minus complanata, ovato-oblonga, breviter acuminata, nonnulla asymmetrica, $0,5 \mathrm{mil}$. 
longa, 0,2 mil. lata, integra, ecostata, cellulis linearibus, paulisper flexuosis, $60-66 \mu$ longis, apicalibus et basilaribus paulo brevioribus, alaribus subquadratis parum conspicuis. Folia perichætialia involuta longius acuminata, integra, ecostata. Capsula in pedicello flexuoso pallido, 3-4 mil. alto subpendula minutissima, humida subglobosa, sicca sub ore vix coarctata, vix 0,5 mil. longa, basi abrupte rotundata. Exos tomii dentes o, 2 mil. longi; endostomii processus e membrana ad medium versus producta æquilongi, integri ; cilia singula paulo breviora. Sporæ minuto, læves, 10-13 $\mu$ crassæ. (Atlas des Mousses de Madagascar, pl. 11 I, fig. 3).

Comores: Mayotte, Mavegani, Marie (Hb. Bescherelle).

Cette espèce, qui paraît être une des plus petites du genre, pourrait être comparée à l'1. intortum $\mathrm{Pal}$. Beauv., des îles austro-africaines; mais celui-ci croît en tapis denses et étendus; ses feuilles sont espacées, étalées ou même parfois un peu squarreuses, nullement aplanies, plus longuement et plus finement acuminées, distinctement dentées; le pédicelle est deux fois plus long, le péristome plus développé, la capsule et les spores plus grosses.

I. appressum Ren. Card.-Pallide lutescenti-viride. Caulis repens, 5-15 mil. longus, ad terram radicibus arcte adfixus, dense pinnatus, ramis perbrevibus, 2-3 mil. longis, apice acutis subdrepanoideis. Folia dense conferta erecta appressa, haud complanata, apicalia paululum subsecunda, o,70 mil longa, ovata, breviter acuminata, ad angulos leviter rotundata-auriculata, subintegra, costis binis sat distinctis, rete denso, vermiculari, cellulis vacuis linearibus (long. 6o-70 $\mu$ ), flexuosis, pro more obtusiusculis, apice papillose prominentibus, basilaribus laxioribus, parenchymatosis, breviter rectangulis infimis multo majoribus rectangulis, alaribus pro more singulis vel binis, inflatis, hyalinis, tenuiter limitatis. Cætera desunt. (Atlas des Mousses de Madagascar, pl. I I , fig. 1 .

Madagascar: Nossi-bé, Marie (Hb. Bescherelle).

Cette espèce, qui possède un peu le port d'un Pterogoniella, se rapproche sous quelques rapports de l'I. Boivini Besch., de l'île Sainte-Marie, mais s'en distingue notamment par la forme des feuilles, par les rameaux légèrement drépanoïdes, par le tissu vermiculaire, celui de la base plus lâche, parenchymateux, et par les angles généralemenl arrondis, composés de I ou 2 grandes cellules dilatées sans cependant former d'oreillettes distinctes.

L'I. Ambreanum Ren. et Card. de Diego-Suarez a aussi quelque analogie avec notre plante par la forme des feuilles et le tissu basilaire ; mais les cellules foliaires n'ont pas de saillies apicales papilliformes, les rameaux sont plus allongés, aplanis, 
les feuilles un peu espacées, plus ou moins étalées, nullement imbriquées, ce qui lui donne un faciès tellement différent que, même à première vue, il n'y a pas de confusion possible.

I. citrinellum Ren. Card (Pl. xx et xix). - Sericeum, lutescenticitrinum. Caulis repens, radiculosus, ad cortices arcte adfixus, I-2 cent. longus, dense ramosus, ramis brevibus, sericeis. Folia erectiuscula, apice subflexuosa, haud complanata, 1,75-2 mil. longa, anguste lanceolata, longe et tenuiter acuminata, cuspidata, jam e basi sinuolata, in dimidio superiore remote et acute dentata, marginibus passim revolutis, costis binis inæqualibus, sat distinctis, cellulis densis, flexuosis, acutis, I 2 - 5 longioribus $(9 \circ \mu)$ quam latioribus, alaribus paucis, breviter rectangulis, interdum 2 vel 3 dilatatis, hyalinis. Cætera desunt.

\section{La Réunion: Rodriguez, I889.}

Cette espèce peut être comparée à l'I. leiotheca Ren. et Card., de la même île; elle en diffère par ses tapis denses d'une belle couleur citron, par ses rameaux nullement aplanis, par ses feuilles plus longues beaucoup plus finement acuminées plus distinctement dentées supérieurement (dents espacées, aiguës, très saillantes), sinuolées jusque près de la base, plus nettement nerviées.

I. Boivini Besch.

Var. terrestre Ren. Card. - Cæspites densi, tapetis ad instar longe lateque extensi, pulchre virides, ramis dense confertis ; folia vix complanata, basi latiora, paulo laxius reticulata, cellulis utriculi primordialis vestigio repletis. (Atlas des Mousses de Madagascar, pl. I Io, fig. 3).

Madagascar : Nossi-Comba, Mad. Réné Caillé, 1892, (Hb. de Poli).

Var. costatum Ren. Card. - Cæspites densi, depressi, virides. Folia haud complanata paulisper subsecunda, majora, longius acuminata, costis binis inæqualibus melius notatis, ad $3 / 4$ folii vel paulo ultra productis, rete vix vel non chlorophylloso. (Atlas des Mousses de Madagascar, pl. 112, fig. 2).

Madagascar: île Sainte-Marie, sur le sable de la plage, Girod Genet, 1898.

I. Maniæ Ren. Par. (Pl. xIx). - Cæspites laxi deplanati luteovirides, nitidi, inferne sordidi. Caulis prostratus 3-4 cent. longus, sat 
robustus, irregulariter subdichotome divisus, parce ramosus, pallide viridis vel lutescens. Folia subdisticha complanata fere patula I $1 / 2 \mathrm{mil}$. longa, e basi ovata oblonga lanceolata sensim acuminata, plus minus longe cuspidata, marginibus planis passim late involutis e basi obsolete et remote, superne acute dentatis serratisve; costa gemella distincte notata interdum crure longiore sat alte producta. Rete denso pallido vermiculari, cellulis sæpius apice obtusis (long. 60-70 $\mu$ ), alaribus perpaucis parvulis quadratis granulosis. Cætera desunt.

Madagascar : province de Betafo, rive droite de la Mania, Gulinon, 1901, (Hb. Paris).

Cette espèce, par son port et sa taille robuste, se distingue à première vue de tous les Isopterygium de la région et même de l'Afrique australe. Elle ressemble à certaines formes saxicoles robustes du Plagiothecium denticulatum d'Europe dont elle diffère d'ailleurs essentiellement par la forme des feuilles et la densité du tissu qui est plutôt celui d'un Isopterygium que d'un Plagiothecium. Les feuilles étalées subdistiques, d'une couleur pâle et d'un aspect parfois scarieux laissent voir la tige robuste d'un vert pâle ou jaunâtre. Cette espèce est voisine de 1 . taxithelioides C. Müll. de l'Himalaya.

\section{Gen. Ectropothecium Mitt.}

E. Perroti Ren. Card.

Madagascar: province de Tamatave, forêt de Fito, PErrot, I897, (Hb. Levier).

Dans cette espèce les nervures sont souvent longues, parallèles, atteignant le milieu de la feuille.

E. occultum Ren. Card.- Monoicum, gracillimum, viride, nitidulum, laxe intricato-cespitosum, aliis muscis intermixtum. Caulis tenuis, repens vel procumbens, $3-4$ cent. longus, ramis patulis plumulosis irregulariter pinnatus. Folia caulina remota, patentia, triangulari-ovata, sensim longe acuminata, subulata, sæpe curvatula, o,7-0,8 mil. longa, $0,25-0,3$ lata, marginibus planis vel inferne plus minus reflexis subrevolutisve, ubique integris aut apicem versus vix subsinuatis, nervis binis brevibus obsoletisve, cellulis longe et anguste linearibus, alaribus perpaucis, brevioribus, subindistinctis ; folia ramea patentia, haud vel vix subsecunda, symmetrica vel curvatula, caulinis angustiora, oblongo- 
lanceolata, sensim longe acuminata, integra, o, 7 0,75 mil. longa, o, 15 0,2 lata. Folia perichætialia erecta, e basi ovata vel oblonga longe acuminato-subulata, integra vel superne obsolete denticulata. Capsula in pedicello purpureo, I5-I $8 \mathrm{mil}$. longo, apice curvato, pendula subglobosa, o,75-I mil. longa, o,6-o, 7 crassa, operculo convexo, acute apiculato. Peristomium lutescens, dentibus intus dense et alte trabeculatis.

\section{La Réunion: Peteull, (Hb. Sebille).}

Cette Mousse rappelle beaucoup, par son port, une petite forme grêle et lâche du Microthamnium aureum Besch., avec laquelle elle croît en mélange intime; il est facile de l'en distinguer à ses feuilles plus longuement acuminées, complètement lisses, et à sa capsule pendante, subglobuleuse. Elle diffère de l'E. regulare C.M. et des espèces voisines par son inflorescence monoïque, son port grêle et ses feuilles étalées, non ou à peine homotropes et entières aux bords.

E. podorrhizum Ren. Card. - Monoicum, inter cespites E. nanocristacastrensis sparse vigens, superne pallide viride, inferne decoloratum. Caulis debilis, flexuosus, i 5-25 mil. longus, inordinate ramosus, apice pro more hamato-curvatus. Folia sat dense conferta, erecta, leviter subsecunda, nitida, ovato-oblonga, breviter acuminata, interdum curvatula, integra, enervia vel subenervia, 1,25 mil. longa, passim paulo ásymmetrica, rete laxiusculo, cellulis mediis lineari-subhexagonis interdum flexuosis, circa 8-longioribus quam latioribus $(65-75 \mu$ longis), acutis, superioribus subilo brevioribus, latiusculis, vacuis, basilaribus laxioribus, alaribus 4-6 dilatatis, hyalinis, rotundato-ellipticis, tenuiter limitatis. Flores masculi haud longe a perichætiis siti, radiculosi ; folia perigonialia ovata, apiculata; antheridia 4-6, paraphysata. Perichætium valde radicans; folia perichætialia ovata, sat subito longe cuspidata, 1,5 mil. longa, summo denticulata. Capsula in pedicello rufo, pallido, flexuoso, tenui, 15-20 mil. longo inclinata vel horizontalis, castanea, oblonga, curvata vel ovata, sub ore plus minus coarctata, collo plicatulo instructa, operculo convexo, oblique apiculato, subrostellato. Exostomii dentes 0,37 mil. longi, transverse striati, superne granulosi, endostomii processus e membrana ad medium dentium producta integri, fere æquilongi ; cilia breviora, nodulosa, singula vel bina in uno coalita vel plus minus cohærentia. Sporæ $12-15 \mu$ crassæ. (Atlas des Mousses de Madagascar, pl. 115 , fig. 2).

Madagascar: Ambatomanga, alt. I 200-1300 mètres Rev. TAlazaC, 1894 .

La place de cette espèce dans le genre Ectropothecium reste encore un peu incertaine. Nos échantilions ont dû être extraits, brin par brin, d'une grosse touffe 
dense d'E. nano-cristacastrensis (C. Müll.) Kindb., et il est difficile, dans ces conditions, d'avoir une idée exacte du port naturel de notre plante, dont le faciès est légèrement drépanoïde. La forme de la capsule varie de l'ovale court à l'oblong, plus allongée que la capsule subglobuleuse des Ectropothecium. En revanche, le péristome est élevé et les cils souvent cohérents deux àdeux comme dans ce dernier genre. La densité du tissu foliaire est à peu près comme dans le genre Isopterygium, chez lequel le péristome est moins élevé. Quelle que soit la place exacte à lui attribuer, notre plante reste très distincte spécifiquemeut.

E. Combæ Ren. Card. - Pallide lutescens. Caulis corticibus emortuis radicibus arcte adfixus, 3-4 cent. longus, irregulariter pinnatus, ramis brevissimis, 2-3 mil. longis. Folia 1,25 mil. longa, laxiuscule conferta, erecto-curvata, paulo subsecunda, oblongo-lanceolata, breviter acuminata, leviter asymmetrica, dorsalia angustiora lanceolata, marginibus integris vel summo apice remote et vix crenulatis, nervis parum distinctis vel nullis, cellulis mediis elongate subhexagonis, 8-longioribus quam latioribus, gramulis chlorophyllosis secus parietes seriatis, utriculo primordiali haud conspicuo. Folia perichætialia multo longiora. longe cuspidata. Capsula in pedicello pallide rufo, 15-18 mil. longo, pendula, ovata vel ovato-oblonga, collo brevi sat distincto instructa. Exostomii dentes o, 4 mil. longi ; endostomii processus e membrana medium versus producta, carinata, æquilongi, anguste lanceolati, interdum carina rimosi ; cilia nodulosa, singula vel bina in uno plus minus coalita. Sporæ 10-12 $\mu$ crassæ. (Atlas des Mousses de Madagascar, pl. 12 I, fig. I).

Madagascar : Nossi Comba, MARIE (Hb. Bescherelle).

Cette plante que l'on pourrait comparer à l'E. spharocarpum (C. Müll.) Besch., de la même région, assez voisin par le port et appartenant également à la section Vesicularia, en diffère par le faciès un peu plus grèle, par les feuilles plus étroites et plus allongées, à peu près énerves, par les cellules ne présentant pas de traces distinctes de l'utricule primordial, seulement granuleuses le long des parois, les moyennes plus longues $(90-\mathrm{r} 20 \mu$ au lieu de $60-70)$ et plus étroites (10-1 $3 \mu$ au lieu de I4-18), enfin par la capsule moins renflée, plus distinctement atténuée en col court, ovale-oblongue, non subglobuleuse.

E. Boivini C. Müll.

Madagascar: Maintirano sur le littoral du Mozambique (Hb. Paris).

E. sphærocarpum C. Müll.

Madagascar: Cercle de Maintirano (Hb. Paris). 
Var. subpiligerum Ren. Par. - A forma normali differt, foliis longius acuminatis subpiliformi-cuspidatis, cellulisque paulo angustioribus, longioribus.

Madagascar: province de Betafo, rive droite de la Mania, Galinon, 190.1 (Hb. Paris).

\section{Gen. Sphagnum Dill.}

S. (Cymbifolia) grandirete Warnst.

Madagascar: province d'Andevorante, Androrangobe, 1901 (Hb. Paris).

S. (Subsecunda) Mathieui Warnst.

Madagascar : Maroantsetra, baie d'Antongil, Mathiev, (Hb. Cardot).

S. (Cuspidata) Ikongoense Warnst.

Madagascar: Ikongo, Dr BESSON, 1892 ; province d'Andevorante, Androrangobe, $190 \mathrm{I}(\mathrm{Hb}$. Paris).

\section{HEPATICA}

Les espèces marquées d'une astérisque figurent déjà dans l'ènumération du Prodrome.

* Frullania affinis Nees. : Madagascar.

F. Densepinnata Steph. : Madagascar.

F. LoRicata Kiaer.: Madagascar.

* F. nodulosa Nees. : Madagascar.

F. ovalifolia Pears. : Madagascar.

F. Renistipula Steph.: Madagascar.

F. subrlana Gottsche: Madagascar.

F. usambarana Steph. : Madagascar.

Ceratolejeunea papuliflora Steph. : Madagascar.

* Eulejeunea isomorpha Gottsche: Grande Comore. 
* Euosmolejeunea trifaria Nees. : Madagascar.

Lopholejeunea borbonica Gottsch. : Madagascar.

Strepsilejeunea decemplicata Steph.: Madagascar.

* Radula Boryana Nees. : Grande Comore.

Bryopteris madagassus Steph. : Madagascar.

Madotheca Hoenneliana Steph. : Madagascar.

* M. cucullistipula Steph. : Madagascar.

M. Montauti Steph. : Madagascar.

M. ovifolia Steph. : Madagascar.

Mastigobryum mascarenum Steph. : Madagascar.

M. Perrotanum Steph. : Madagascar.

Cephalozia grossitexta Steph.: Madagascar.

Chiloscyphus oblongifolius Mitt. : Madagascar.

Ptrchanthus striatus Nees: Madagascar :

* Plagioghila angusta Ldb. : Madagascar.

P. Crollei Steph.: Madagascar.

* P. comorensis Steph.: Grande Comore.

P. Hildebrandti Steph.: Madagascar.

P. divergens Steph. : Madagascar.

P. Flagellaris Steph.: Madagascar.

* P. Repanda Lindbg. : Madagascar.

P. Perrotana Steph. : Madagascar, Grande Comore.

P. valida Steph. : Madagascar.

Thylmanthus Lespagnoli Steph. : Madagascar.

T. Madagascariensis Steph.: Madagascar.

Symphiomitra glossophylla Spruce : Grande Comore.

\section{$A D D E N D A$}

Fissidens Lacouturei Thér. - Voisin de $F$. exasperatus Ren. Card.: en diffère par ses feuilles plus larges, à lame vraie proportionnellement plus longue, par le tissu papilleux et les cellules marginales faiblement saillantes.

Madagascar: Betsileo; Rev. VILlaume, Igo6 (Hb. Lacouture). 
Espèces collectées en 1903-1904 dans les îles austro-africaines par lé Dr A. Voeltzkow et déterminées par V. F. Brotherus (A).

Microdus Limosus Besch. - Madagascar: Fénerive.

Holomitrium vaginatum Brid. - Maurice.

Leucoloma (Albescentia) candidum Broth n. sp. - Sainte-Marie de Madagascar ( $\mathbf{I}$ ).

L. sinuosulum C. Müll. - Maurice.

L. Isleanum Besch. - Maurice.

L. BIFIdum Brid. - Maurice.

L. Chrysobasilare C. Müll. - Grande Comore, alt. igoo mètres.

L. Crepini Ren. Card. et var. orthothecioides (Besch.) Ren. Maurice.

L. Sancte Marie Besch. - Madagascar: Sakana.

L. sinuosum Brid. - Maurice.

Campylopus Boryanus Besch. - Maurice.

C. Borvini Besch. - Maurice.

C. Robillardi Besch. - Maurice.

C. Flagryi Ren. Card. - Madagascar: Alaotra-Fenerive.

Campylopus Voeltzkowii Broth., n. sp. - Sainte-Marie de Madagascar (2).

C. Pallescens Besch. - Grande Comore, alt. Igoo mètres et cratère, 2300 mètres; Maurice.

C. polytrichoides de Not. - Grande Comore, alt. Igoo mètres et cratère, 2300 mètres; Maurice.

Leucobryum Isleanum Besch. - Comores, Moheli (70o mètres); Madagascar; Sakana.

Cardotia Boiviniana (Besch.) Card. - Sainte-Marie de Madagascar.

Octoblepharum albidum (L.) Hedw. - Maurice, Madagascar : Fénérive.

Leucophanes Hildebrandti C. M. - Comores, Anjouan (80o mètres).

L. Rodriguezir C. Müll. - Maurice; Madagascar: Tamatave.

(A) Musci Voeltzkowiani. Ein Beitrag zur Kenntnis der Moosflora der ostafricanischen Inseln von V. F, Brotherus. Stuttgart, 1908, 3 planches.

(i) Caractérisé, d'après l'auteur par ses cellules supérieures faiblement papilleuses. Doit être voisin d'une forme de Maurice conservée dans mon herbier sous.le nom de L. sinuosulum C. Müll. var. sublave Ren.

(2) (Trichophylla). Comparé aux C. bicolor Hsch. et C. atroluteus (C. Müll.) dont il diffère par la structure des feuilles. 
Fissidens Marjei (Besch.) Broth. - Madagascar: Sakana.

F. ovatus Brid. - Maurice.

Syrrhopodon apertifolius Besch. - Maurice.

S. glaucophyllus Ren. Card. - Madagascar, Est.

S. Mauritianus C. M. - Maurice.

Calymperes Sancte Marie Besch. - Madagascar: Tamatave.

C. Mariei Besch. - Madagascar: Sainte-Marie; Fénérive.

Hyophila Potierir Besch. - Sainte-Marie de Madagascar.

Trichostomum circinnatulum Broth. n. sp. - Grande Comore (1900 mètres) ( 1 ).

Macromitrium lanceolatum Broth. n. sp. - Maurice (2).

M. calomicron Broth. n. sp. - Maurice (3).

M. Mauritianum Schwgr. = Maurice.

M. Voeltzkowii Broth. n. sp. - Maurice. (4)

Funaria calvescens Schwgr. - Maurice; Grande Comore (Igoo mètres).

Brachymenium microcarpum Broth. n. sp. - Maurice (5).

B. submicrocarpum Broth. n. sp. - Maurice (6).

Bryum argenteum L. - Maurice; Grande Comore (Igoo mètres).

B. madagasso-ramosum Broth. n. sp. - Madagascar, Sakana (7).

B. Voeltzkowii Broth. n. sp. - Madagascar, Sakana (8).

Rhizogonium mauritianum Hpe. - Maurice.

Philonotis comorensis C. M. - Grande Comore. (Igoo mètres).

(1) (Oxystegus). Comparé au $T$. circinnatum (Besch.) Broth. dont il diffère par sa tige courte et la forme des feuilles.

(2) (Cometium). Voisin du $\boldsymbol{M}$. scleropodium Besch. S'en distingue par la forme des feuilles contournées en spirale à l'état sec, squarreuses à l'état humide, carénéesconcaves, lancéolées, aiguës, à marges planes très entières, nervure épaisse rousse, lisse, excurrente en une pointe subdenticulée. Cellules grandes arrondies non épaissies, presque lisses.

(3) (Goniostoma). Comparé au M. (Dasymitrium) borbonicum (Besch.) Broth. dont il diffère par le pédicelle très court.

(4) (Leiostoma). Caractérisé, d'après l'auteur, par ses feuilles étroitement contournées. Pédicelle long de 8 mill.; capsule ovale microstome, lisse. Peristome réduit à une membrane rudimentaire. petite.

(5) (Orthocarpus). Diffère de ses congenères de l'Afrique par sa capsule très

(6) Diffère du B. microcarpum Broth. par sa taille plus robuste, ses feuilles plus longuement aristées, par son pédicelle 2 fois plus long.

(7) (Rosulata). Voisin du B. ramosum (Hool.) Mitt., mais feuilles plus étroitement marginées.

(8) (Rosulata). Diffère du B. ramosum (Hook.). par sa taille plus robuste, ses tiges plus allongées et ses feuilles plus larges. 
P. mauritiana Angstr, - Maurice.

P. oвtusata C. M. - Madagascar: Fénérive.

Pogonatum Belangeri (C. M.) Besch. - Maurice.

P. gracilifolium Besch. - Madagascar: Alaotra, Fénérive.

Polytrichum piliferum Schreb. Var. australe Ren. Card. - Grande Comore. (1900-230o mètres).

P. comorense C. M. - Grande Comore (1900 mètres).

P. CAlopogon Besch. - Maurice.

P. commune L. - Grande Comore (Igoo mètres); Maurice:

Jagerina formosa Besch. - Maurice.

J. stolonifera C. M. - Comores Anjouan (80o mètres).

Pilotrichella ampullacea C. M. - Grande Comore (I900 mètres).

Aerobryum lanosulum C. M. - Madagascar.Est.

Papillaria Robillardi C. M. - Maurice.

Neckera Comora C. M. - Comores: Moheli (700 mètres).

Homalia Valentini Besch. - Maurice.

Porotrichum Comorense C. M. - Maurice.

P. Robillardi C. M. - Maurice.

Leptohymenium fabronoides C. M. - Grande Comore.

Cillicostella lacerans C. M. - Maurice; Madagascar : Tamatave.

Hypopterygium laricinum (Hook.) Brid. -- Madagascar: Sakana.

Rhacopilum mauritianum C. M. - Maurice.

R. angustistipulaceum. C. M. - Comores: Anjouan (iooo mètres).

Hypnum (Ctenidium) mauritianum Broth. n. sp. - Maurice (r).

Etropothecium tamatavense Broth. n. sp. - Madagascar: Tamatave (2).

E. Galerulatum Duby. - Madagascar: Sakana.

Taxithelium (Polystigma) Voeltzkovii Broth. n. sp. - Maurice (3).

Taxithelium Letum Ren. Card. - Madagascar: Sakana.

Pterogoniella Schimperi Besch. - Maurice.

P. Madagascariensis (Brid,) - Madagascar: Fénérive, Tamatave.

P. Diversifolia Ren. Card. - Madagascar Est.

(1) (Ctenidium). Comparé par l'auteur au H. (Ctenidium) Leveilleanum Doz.

(2) (Cupressina). Espèce très distincte, voisine de E. monumentorum Duby, mais qui en diffère par ses feuilles peu courbées et ses cellules très étroites.

(3) (Polystigma). Caractérisé, d'après l'auteur, par l'exiguité de toutes ses parties et par son port semblable à celui de Isopterygium intortum P. B. 
Espèces collectées à Madagascar par. MM. Catat, Trattoux, Leblanc, Coudert, Cabanes. (Herb. Muséum de Paris).

Dicranella Pervilleana Besch. - Manjakandriana.

Leucoloma dichelymoides C. Müll. - Volon-Kaso.

Dicranoloma scopareolum (C. M.). - Volon Tany.

Campylopus filescens Ren. Card. - Manjakandriana.

C. subcomatus Ren. Card. - Manjakandriana.

Leucobryum madagassum Besch. - Volon Tany.

L. Boryanum Besch. - Manjakandriana.

Fissidens Arbogasti Ren. Card. - Manjakandriana.

Syrrhopodon Chenagoni Ren. Card. - Volon Kazo.

Coleochetium subappendiculatum Broth. n. sp. - Volon Kazo.

C. Renauldir Broth n. sp. - Sine loco.

Polytrichum subformosum Besch, - Manjakandriana.

P. Longissimum C. M. - Volon Tany; Manjakandriana.

Rutenbergia madagassa Geh. et Hpe. - Volon Kazo.

Renauldia Hildebrandtielloides (Ren. Card.) C. M. - Volon Kazo.

Pilotrichella biformis Hpe. - Volon Kazo.

Papillaria Boivini Besch. var. macratis (C. M.) Ren. - Volon Kazo.

P. Patentissima (C. M.) Fleisch. - Manjakandriana.

Leptohymenium Ferriezii (Marie) Vohilema; circa Tullear (S. W.).

Thuidium aculeo serratum Ren. Card. - Volon Tany; Volon Kazo.

Pseudoleskea subfilamentosa Kiær. Ambohimanga.

Ectropothecium arcuatum Ren. Card, - Vohilema.

Espèces collectées en 1905 à Anjouan (Comores) par M. Lavanchie (Hb. Museum de Paris).

Leucoloma Comora Ren., L. Isleanum Besch,, Campylopus Hildebandtii C. M., Leucobryum Isleanum B., L. Comorense C. M., Leucophanes Hildebrandtii C. M., L. Rodriguezii C. M., Octoblepharum albidum Hedw., Macromitrium subpungens Hpe., Bryum Commersoni Schwgr., Bryum homalobolax C. M., Rhizogonium spiniforme, Jagerina stolonifera C. M., Hildebrandtiella endotrichelloïdes C. M., Neckera Comora C. M., Leptohymenium Ferriezii Marie, Pilotrichella ampullacea C. M., P. pseudo imbricata C. M., Aerobryum lanosulum C. M., Ectropothecium regulare C. M., Taxithelium serratum Ren. Card. 
Ces diverses listes fournissent l'appoint suivant aux différentes îles de notre Domaine:

$I^{\circ}$ Pour l'ensemble du Domaine i 5 espèces dont 13 nouvelles.

$2^{\circ}$ Pour Madagascar 1 I espèces dont 5 nouvelles.

$3^{\circ}$ Pour Maurice 3 espèces dont 7 nouvelles.

$4^{\circ}$ Pour la Grande Comore 7 espèces, ce qui porte leur nombre total à 64 espèces.

$5^{\circ}$ Pour Moheli 2 espèces.

$6^{\circ}$ Pour Anjouan 8 espèces, ce qui porte leur nombre total à 62 espèces. 


\title{
NOTE ADDITIONNELLE
}

\author{
AJOUTÉE PENDANT L'IMPRESSION
}

Grimmia madagassa Ren. Card. - Nigro-viridis. Caulus erectus 12-20 mill. longus, simplex vel parce et breviter ramosus. Folia elongate lanceolata, sensim et longe acuminata, pleraque pilo brevi parce denticulato vel subintegro instructa, integerrima, uno margine late reflexo, altero plano, costa rotundata percurrente, cellulis inferioribus laxiusculis lutescentibus rectangulis, parietibus haud vel parum sinuosis, sequentibus subquadratis parietibus incrassatis cæteris omnibus in $2 / 3$ vel $3 / 4$ superioribus folii ubique bistratosis, minutis, obscuris, valde irregularibus, subquadratis, subrotundatis vel transverse dilatatis. Cætera desunt.

Madagascar: plateau central (Hb. Mitten).

Cette espèce qui nous a été obligeamment communiquée par Mrs E. G. Britton ne portait dans l'herbier Mitten aucun nom d'espèce ni de collecteur. En l'absence de fructifications, il est difficile de lui assigner une place précise. Elle est remarquable par ses feuilles entièrement bistrates dans les $2 / 3$ ou même les $3 / 4$ supérieurs et n'est guère comparable aux espèces du Cap connues jusqu'à ce jour. Le genre Grimmia n'était pas encore représenté à Madagascar.

Amblystegium chalaropelma C. Müll. in hb.

Madagascar: Ost Imerina; leg. Hildebrandt, Dec. I880, n 2076.

Monoïque. Touffes denses, tiges couchées, rameaux serrés, courts. Feuilles presque étalées, long. $1 \mathrm{~mm} \mathrm{~m} / 4$, oblongues-lancéolées, longuement rétrécies acuminées, presque subulées, entières ou çà et là, faiblement crénelées; nervure atteignant l'extrémité de la subule; cellules vides subhexagones-allongées $3-6$ fois aussi longues que larges, les alaires carrées peu nombreuses. Folioles périchétiales dressées-appliquées, raides, fortement plissées, plus longues $(2-2$ I/4 mill. $)$ lancéolées, rétrécies dès la base et brièvement subulées, marges entières plus ou moins ondulées ou légèrement crénelées dans le haut, nervure forte, excurrente; tissu délicat hyalin, cellules 
allongées hexagones sublinéaires 8-ı fois aussi longues que larges. Pédicelle long de 20-25 mill. très flexueux. Capsule courte I 1/4 mill., fortement arquée, opercule convexe apiculé. Dents de l'exostome lancéolées, jaunes et striées transversalement dans lamoitié inférieure, décolorées et finement papilleuses dans la moitié supérieure; endostome jaunâtre finement granuleux, processus légèrement ouverts sur la carène, I-2 cils fortement appendiculẻs, papilleux. Spores granuleuses relativement grosses.

Description faite d'après l'échantillon original de C. Müller, conservé au Muséum de Berlin. Le genre Amblystegium n'est encore représenté à Madagascar que par cette seule espèce qui ressemble par le port à $A$. serpens $L$. d'Europe, mais en reste bien distincte par sa nervure longue, ses feuilles subulées, par ses folioles périchétiales et sa capsule courte.

\section{Ectropothecium (Vesicularia) mmmundum (C. Müll. in hb.) Ren.}

\section{Madagascar : Ost Imerina ; leg. Hildebrandt, Janvier I 88. I, n ${ }^{0} 2077$.}

Monoïque. Tige couchée, rameaux raides dressés, long. $1 / 2-1$ cent., simples ou nunis de rares rameaux très courts. Feuilles presque étalées, d'apparence subdistiques, nullement flexueuses, petites (long. $3 / 4$ mill.), les latérales légèrement asymétriques ovales ou oblongues, contractées en une pointe courte, planes, entières presque ẻnerves à marges planes, cellules courtes subhexagones rhombées, remplies de granulations, les dorsales plus insensiblement rétrécies et plus longuement acuminées-cuspidées, concaves, munies de deux petites nervures, cellules plus allongées vides. Pédicelle long de 13 mill., flexueux. Capsule rousse globuleuse pendante.

Description faite d'après l'échantillon original de Carl Müller, conservé au Muséum de Berlin. Cette espèce figure dans le Prodrome (page 263) sous le nom de Hyrpum mundum C. Müll. in Wright, Journ. of. bot. 1888 (nomen nudum). Dans la même énumération de Wright figure aussi, sous le nom de Hypnum latocaspitosum C. Müll., (Prodrome page 263) une plante qui, d'après l'examen que j’ai pu faire de l'échantillon original de C. Müll., ne differé pas de Hypnum cupressiforme L. 


\title{
ATLAS DES MOUSSES DE MADAGASCAR
}

\author{
EXPLICATION DES PLANCHES
}

Pl. 1. - I. Sporledera laxifolia Ren. et Card. - a a', feuille caulinaire $\times 24 . b$, cellules moyennes $\times 170 . c$, cellules basilaires $\times 170 . d d^{\prime}$, sommet de la feuilles $\times 300 . e$, capsule $\times 4^{\circ}$. $f$, sommet de la capsule et spores $\times 170$. $g$, moitié d'une capsule jeune avec la coiffe encore adhérente à la paroi capsulaire $\times 80$. $h$, spores $\times 300$. -2 . Dicranella Polii Ren. et Card. $-a a^{\prime}$, feuille caulinaire $\times 24 . b$, cellules basilaires $\times 300$. c cellules moyennes $\times$ $300 . d$, sommet de la feuille $\times 300 . e e^{\prime}$, dent du péristome $\times 170 . f$, spores $\times$ 300 .

Pl. 2. - Trematodon lacunosus Ren, et Card. - $a$ a', feuille caulinaire $\times 24 . b$, cellules basilaires $\times 300$. $c$, tissu vers les $2 / 3$ supérieurs $\times 300 . d$, sommet de la feuille $\times 300$. $e$ e', capsule $\times 12 . f$, dent du péristome vers son milieu $\times 300 . g$, partie supérieure d'une dent $\times 300 . h$, spores $\times 300$.

P1. 3. - I. Trematodon platybasis C. Müll. - $a$ a', feuille caulinaire $\times 24 . b$, tissu basilaire $\times 300 . c$, sommet de la feuille $\times 300 . d d$, Capsule $\times$ 12. - 2. Leucoloma bifidum Brid. $-a a^{3}$, Feuille caulinaire $\times 24 . b$, feuille d'une innovation grêle $\times 24, c$, tissu basilaire $\times 300 . d$, oreillettes $\times 170 . e$, tissu vers le milieu de la feuille $\times 300$, montrant le limbe hyalin, les cellules membraneuses et les cellules papilleuses. $f f^{\prime}$, sommet de la feuille $\times 170 . g$, limbe hyalin 4-6 sérié $\times 300$.

Pl. 4.- 1. Leucoloma subbifidum Ren. $-a a^{\prime} a^{\prime \prime}$, feuille caulinaire $\times 24$. $b$, sommet de la feuille $\times$ i 7o. $c$, nerwure $\times 170 . d$, oreillette (partie) et tissu membraneux basilaire $\times 300$. $e$, tissu vers le milieu de la feuille $\times 300 . f$, groupe de cellules internes $\times 300 .-2$. Leucoloma Leperyanchei Besch. $a a^{\prime} a^{\prime \prime}$, feuille caulinaire $\times 24 \cdot b \cdot b^{\prime}$ sommet de la feuille $\times$ r $70 . c$, nervure $X$ 170. $d$, tissu vers le milieu de la feuille $\times 300 . e$, groupe de cellules internes $\times$ 30o. - 3. Leucoloma squarrosulum C. M. $a a^{\prime}$, feuille caulinaire $\times 24, b b^{\prime}$, sommet de la feuille $\times 170, c$, oreillette $\times 170 . d$, tissu pris vers le milieu de la feuille $\times 300$. e, groupe de cellules internes $\times 300$. 
P1. 5. - I. Leucoloma subbiplicatum R. C. $a a^{\prime} a^{\prime \prime}$, feuille caulinaire $\times 24$. $b$, sommet de la feuille $\times$ i 70 . $c$, tissu pris vers le milieu de la feuille $\times 300$. $d$, oreillette $\times 170$. $e$. groupe de cellules internes $\times 300 .-2$. Leucoloma procerum Ren. - $a a^{\prime}$, feuille caulinaire $\times 24 . b b^{\prime} b^{\prime \prime}$, sommet de la feuille $\times$ i 7o. $c$, tissu basilaire avec fragment d'oreillette $\times 300, d d^{\prime} d^{\prime \prime}$ 'bord de la feuille et margo hyalin $\times 300$. $e$, tissu membraneux basilaire $\times 300 . f$, tissu pris vers la base de l'acumen $\times 300 . g$, tissu pris un peu au-dessus de la base $\times 300$.

Pl. 6. - I. Leucoloma chrysobasilare, C. Müll. - a. feuille caulinaire $\times$ 24. $b b^{\prime}$, sommet de la feuille $\times 170 . c$, tissu basilaire juxtacostal $\times 300 . d d^{\prime} d^{\prime \prime}$ margo hyalin $\times 300, e$, groupe de cellules internes $\times 300 . f$, tissu basilaire membraneux $\times 300$. - 2. Leucoloma mafatense Ren. - $a a^{\prime} a^{\prime \prime}$, feuille caulinaire $\times 24 . b b^{\prime} b^{\prime \prime} b^{\prime \prime}$, sommet de la feuille $\times 170 . c$, oreillette $\times 170$. $d d^{\prime}$ margo hyalin $\times 300 . e$, groupe de cellules internes $\times 300$.

Pl. 7. - I. Leucoloma subchrysobasilare C. Müll. - a, (Borgen) feuille caulinaire $\times 24, b$, sommet de la feuille $\times 1$ 70. $c$, oreillette $\times$ i 7o. $d$, margo hyalin et tissu membraneux $\times 300 . e e^{\prime}$ (Talazac) feuille caulinaire $\times 24 . g$, tissu pris vers le milieu de la feuille $\times 300 . h$, tissu membraneux suprabasilaire $\times 300, k$, cellules internes un peu au-dessus de la base $\times 300 . l$, cellules internes vers le milieu de la feuille $\times 300\{\mathrm{I}\},-2$. Leucoloma silvaticum Ren.$a a^{\prime} a^{\prime \prime}$, feuille caulinaire $\times 24 . b b^{\prime}$, sommet de la feuille $\times 170, c$, nervure $\times$ 170. $e$, margo hyalin et tissu membraneux $\times 300 . f$, cellules internes moyennes $\times 300 .-3$. Leucoloma Sancto Maria, Besch. - $a a^{\prime} a^{\prime \prime}$ feuille caulinaire $\times 24 . b$, sommet de la feuille $\times 170$. $c$, oreillette et tissu basilaire $\times 170, d$, margo hyalin $\times 300$. e, cellules internes à la base de la subule $\times 300$.

Pl. 8. - I. Leucoloma Thuretii, Besch. $-a a^{\prime}$, feuille caulinaire $\times 24 . b b^{\prime}$, sommet de la feuille $\times 170$. $c$, oreillette $\times 170 . d d^{\prime}$ margo hyalin et tissu membraneux 30o. $e$ ' , cellules internes $\times 300 .-2$. Leucoloma cinclidotiö̈des, Besch. $-a a^{\prime}$, feuille caulinaire $\times 24 . b$, sommet de la feuille $\times 170$ $c c^{\prime}$, oreillette $\times 170 . d d^{\prime}$, margo hyalin $\times 300$, $e$, cellules internes $\times 300$. 3. Leucoloma gracilicaule (C. Müll.). $a a^{\prime}$, feuille caulinaire $\times 24 . b b^{\prime}$, sommet de la feuille $\times$ i $70 . c$, oreillette $\times$ I 70. $d$, margo hyalin $\times 300 . e$, tissu membraneux vers la base $\times 300 . f$, tissu basilaire juxtacostal $\times 300$.

P1. 9. - 1. Leucoloma Comora, Ren. - $a a^{\prime}$, feuille caulinaire $\times 24$. $b$, sommet de la feuille $\times$ i $70 . c$, oreillette $\times 170, d$, margo hyalin $\times 300$. $e$, tissu pris vers le tiers inférieur de la feuille $\times 300 . f$, cellules internes moyennes $\times 300 . g$, subule vers son milieu $\times 300 . h$, tissu basilaire juxtacostal $\times 300,-2$. Leucoloma Seychellense, Besch. $-a a^{\prime}$, feuille caulinaire $\times 24$. $b$, sommet de la feuille $\times 170 . c$, oreillette $\times 170 . d d^{\prime}$, margo hyalin $\times 300, e$, tissu basilaire membraneux $\times 300$.

(r) Cette plante récoltée par Talazac a été distinguée plus tard sous le nom de $L$. ochrobasilare Ren. et décrite dans le supplément du Prodrome. 
Pl. ro. - I. Leucoloma Crepini, Ren, et Card. - $a$ ' feuille caulinaire $\times 24$. $b$, oreillette $\times 170, c$, margo hyalin et tissu membraneux $\times 300 . d$, var. orthothecioides, feuille caulinaire $\times 24 . e$, tissu membraneux basilaire de cette var. $\times 300 .-2$. Leucoloma Talazaccii, Ren. et Card. - a a' feuille caulinaire $\times 24 . b b^{\prime}$ sommet de la feuille $\times 170 . c$, oreillette $\times 170 . d d^{\prime}$ margo hyalin $\times 300$. $e e^{\prime}$, cellules basilaires juxtacostales $\times 300,-3$. Leucoloma tuberculosum, Ren. - $a a^{\prime}$ feuille caulinaire $\times 24 . b b^{\prime}$, sommet de la feuille $\times$ i $70 . c$, oreillettes $\times 170$. $d d^{\prime}$ margo hyalin et tissu membraneux $\times$ 30o. $e$, tissu basilaire membraneux $\times 300 . f$, cellules internes moyennes $\times$ 300. g, papilles dorsales $\times 500$.

Pl. I1. - Leucoloma Grandidieri, Ren et Card. - $a a^{\prime}$, feuille caulinaire $\times 24 . b$, sommet de la feuille $\times 170 . c$, oreillette $\times 170 . d d^{\prime} d^{\prime \prime}$, margo hyalin $\times 300 . e$, cellules suprabasilaires $\times 300 . f$, coupe transversale d'une feuille vers le milieu $\times 525$.

Pl. I2. - I. Leucoloma ccespitulans, C. Müll. - $a a^{\prime} a^{\prime \prime}$, feuille caulinaire $\times 24 . b$, sommet de la feuille $\times$ i $70 . c$, oreillette $\times$ i $70 . d$, tissu pris vers le milieu de la feuille $\times 300$. $e$, margo hyalin $\times 300 . f$, tissu suprabasilaire juxtacostal $\times 300 . g$, cellules internes $\times 300$. -2 . Leucoloma amblyacron, C. Müll. - $a a^{\prime} a^{\prime \prime}$, feuille caulinaire $\times 24 . b b^{\prime}$, sommet de la feuille $\times 170$. $b^{\prime \prime}$ $i d . \times 300 . c$, oreillette $\times 170 . d$, tissu vers le milieu de la feuille $\times 300 . e$, margo hyalin $\times 300 . f$, cellules internes moyennes $\times 300$. - Leucoloma subcospitulans, Besch. $-a a^{\prime}$, feuille caulinaire $\times 24 . b$, sommet de la feuille $\times$ 170. $c$, oreillette $\times$ i 70. $d$, margo hyalin $\times 300$. $e$, cellules internes $\times 300$.

P1. 13. - 1. Leucoloma cirrosulum, Ren. - a a' $a$ ", feuille caulinaire $\times$ 24. $b b^{\prime}$, sommet de la feuille $\times 170 . b^{\prime \prime}, i d . \times 300 . c$, oreillette et tissu basilaire $\times 170 . d$, subule vers son milieu $\times 300$. e e margo hyalin $\times 300 . f$, tissu basilaire $\times 300 . g g^{\prime}$, cellules internes $\times 300 .-2$. Leucoloma cuneifolium, Hpe. - $a a^{\prime} a^{\prime \prime} a^{\prime \prime}$, feuille caulinaire $\times 24 . b$, sommet de la feuille $\times$ I70. $c c^{\prime}$, oreillette $\times 170 . d$, margo hyalin $\times 300 . e e^{\prime}$, tissu basilaire $\times 300$. $f$, cellules internes $\times 300 .-3$. Leucoloma Ambreanum, Ren. et Card. $a a^{\prime}$, feuille caulinaire $\times 24, b$, sommet de la feuille $\times$ i $70, c$, oreillette $\times 170$. $d$, margo hyalin et tissu vers le milieu de la feuille $\times 300 . e$, tissu basilaire $\times$ 300. $f f^{\prime} f^{\prime \prime}$, cellules internes $\times 300$.

P1. 14. - 1. Leucoloma delicatulum, Ren. $-a a^{\prime} a^{\prime \prime}$, feuille caulinaire $\times 24$. $b b^{\prime} b^{\prime \prime} b^{\prime \prime}$, sommet de la feuille $\times 170 . c$, oreillette $\times 170 . c^{\prime} i d . \times 300 . d$, tissu vers le milieu $\times 300$. $e$, cellules internes $\times 300 . f$; subule vers son milieu $\times$ 30o. - 2. Leucoloma convolutaceum, Ren. $-a a^{\prime} a^{\prime \prime}$ feuille caulinaire $\times 24$. $b$, sommet de la feuille $\times$ I $70, c$, oreillettes $\times 170, i d . \times 300 . d$, margo hyalin $\times 300$ et cellules internes vers la base de la sulule $\times 300 . e$, subule vers son milieu $\times 3$ oo. -3 . Leucoloma sinuosulum C. Müll. $-a$, feuille caulinaire $\times$ $24 . b b^{\prime}$ sommet de la feuille $\times 170 . c$, oreillette $\times 1$ 7o. $d$, margo hyalin $\times 300$. $e$, cellules internes $\times 300$. $f$, foliole périchétiale $\times 24$. 
P1. 15. - 1. Leucoloma candidulum, C. Müll. - $a$ a', feuille caulinaire $\times 24, b$, sommet de la feuille $\times 170 . c c^{\prime}$ oreillette $\times$ i $70, d$, margo hyalin $\times$ 300 . $e$, tissu suprabasilaire $\times 300 .-2$. Leucoloma Isleanum, Besch. $-a a^{\prime}$, feuille caulinaire $\times 24 . b b^{\prime}$, sommet de la feuille $\times 170 . b^{\prime \prime} i d . \times 300$. $c$, oreillette $\times$ i 7o. $d$, margo hyalin $\times 300$. $e$, cellules internes $\times 300$. - Var. subtortille, Ren. $-f f^{\prime}$, feuille caulinaire $\times 24 . g$, sommet de la feuille $\times 170$. $h$, oreillette $\times 170 . i$, margo hyalin $\times 300 . k$, cellules internes $\times 300 . l$, tissu vers la base de l'acumen $\times 300$. -3 . Leucoloma persecundum, C. Müll. $a a^{\prime}$, feuille caulinaire $\times 24 . b b^{\prime}$, oreillettes $\times 170 . c$, sommet de la feuille $\times$ I 70. $d$, margo hyalin $\times 300 . e e$ ' cellules internes $\times 300 . f$, cellules basilaires $\times 300,-$ Var. Perroti, Ren. $g$, feuille caulinaire $\times 24 . h$, oreillette $\times 170$. $i$, margo hyalin $\times 300 . k$, tissu basilaire $\times 300$. $l$, cellules internes $\times 300$.

Pl. 16. - 1. Leucoloma albocinctum, Ren. et Card. (Sainte-Marie de Madagascar). $-a a^{\prime}$, feuille caulinaire $\times 24 . b$, sommet de la feuille $\times$ i $70 . c$, oreillettes $\times 170, d$, margo hyalin $\times 300$. $e$, tissu basilaire $\times 300 . f$, cellules internes $\times 300 .-$ (Alakaty, Madagascar). $-a$ " $a$ "', feuille caulinaire $\times 24$ $b$ ', sommet de la feuille $\times 1$; $0 . b^{\prime \prime}, i d . \times 300 . d^{\prime}$, margo hyalin et cellules internes $\times 300,-2$. Leucoloma pumilum, C. Müll. $-a a^{\prime}$, feuille caulinaire $\times$ 24. $b b^{\prime}$, sommet de la feuille $\times$ i $70 . c$, oreillettes $\times$ i $70 . d$, margo hyalin et tissu marginal $\times 300$. $e$, tissu basilaire membraneux $\times 300 . f$, cellules internes $\times 300 .-3$. Leucoloma capillifolium Ren. $-a a^{\prime}$, feuille caulinaire $\times$ 24. $b b^{\prime}$, sommet de la feuille $\times 170$. $c$, oreillettes $\times$ r7o. $d$, margo hyalin et cellules marginales $\times 300$. e, cellules internes $\times 300$.

P1. 17. - 1. Leucoloma sinuosum, Brid. $-a$, feuille caulinaire $\times 24$ (type). $a^{\prime}$, id. var. setifolium $\times 24 . b b^{\prime}$, sommet de la feuille $\times 170 . c$, oreillettes $\times$ I $70 . d$, margo hyalin et cellules marginales $\times 300, e e^{\prime}$, cellules internes $\times$ 30o. -2 . Leucoloma fuscifolium, Besch. $-a a^{\prime}$, feuille caulinaire $\times 24 . b b^{\prime}$, sommet de la feuille $\times 170 . c$, oreillette $\times 170, d d^{\prime}$ margo hyalin et cellules marginales $\times 300 . e$, cellules basilaires $\times 300$. $f$, cellules internes $\times 300$. 3. Leucoloma Dubyanum, Besch. - $a$ ', feuille caulinaire $\times 24 . b$, sommet de la feuille $\times$ i $70 . c c^{\prime}$, margo hyalin et cellules internes $\times 300 . d$, nervure $\times$ I $70 . e$, cellules basilaires $\times 300$.

P1. 18. - 1. Leucoloma Boivini, Besch. - $a$, feuille caulinaire $\times 24 . b$, sommet de la feuille $\times$ i $70 . c$, oreillette $\times$ I $70 . d$, margo hyalin $\times 300 . e$, cellules basilaires $\times 300 . f$, cellules vers le milieu de la partie large $\times 300 . g$, cellules juxtacostales moyennes $\times 300$. $h$, base de la subule $\times 300$. $i$, cellules supérieure de la subule $\times 300$. - 2. L. Boivini var. Angasiza Ren. - $a$, feuille caulinaire $\times 24, b b^{\prime}$, sommet de la feuille $\times 170 . c$, oreillette $\times 170 . d$, margo hyalin $\times 300$. $e$, cellules basilaires $\times 300 . f$, cellules internes moyennes juxtacostales $\times 300 . g g^{\prime}$, tissu vers le milieu de la subule $\times 300$. $h$, fraction de la subule vers son milieu $\times 300$. 
P1. 19. - Leucoloma Rutenbergii C. Müll. $a a^{\prime}$, fenille caulinaire $\times 24$. $b$, sommet de la feuille $\times 170 . c$, oreillette $\times 170 . d d^{\prime}$, margo hyalin et cellules marginales $\times 300 . f$, cellules juxtacostales vers. le milieu de la partie élargies $\times 300 . g$, cellules internes vers la base de la subule $\times 300 . h$, cellules internes de la subule moyennes $\times 300 . i$, foliole périchétiale $\times 24 . j$, sommet de la foliole périchétiale $\times 170 . k$, paraphyse $\times 170 . l$, coiffe $\times 24 . m$, archégone $\times 40, n$, spores $\times 300 . p$, fragment de la feuille pris à la base de la subule et montrant les rugosités dorsales $\times 300 . q$, fragment du péristome oblitéré $\times$ I 7o. $r$, (var. Perroti) oreillette $\times$ I $70 . s$, (id.) dents du péristome $\times$ I 70 .

P1. 20. - 1. Leucoloma arbusculum C. Müll, $-a$, sommet d'une feuille caulinaire $\times$ I $70 . b$, oreillette $\times$ I $70 . c$, margo hyalin $\times 300 .-2$. Leucoloma dichelymoides C. Müll. - $a a^{\prime}$, feuille caulinaire $\times 24 . b b^{\prime}$, sommet de la feuille. $c$, oreillette $\times 170 . d d^{\prime}$, margo hyalin et cellules marginales $\times 300 . e$, cellules basilaires juxtacostales $\times 300 . f f^{\prime}$, tissu basilaire $\times 300 . g$, cellules vers le milieu de la partie élargie de la feuille $\times 300 . h$, cellules internes vers la base de la subule $\times 300$. - 3. Leucoloma Sieber $i,-a a^{\prime}$, feuille caulinaire $\times 24 . b$, sommet de la feuille $\times 170 . c$, oreillette et tissu basilaire $\times 170 . d$, margo hyalin $\times 300$. $e$, cellules supérieures $\times 300, f$, dent du péristome $\times$ I 70 et fragment $\times 300$.

Pl. 21. - I. Leucoloma dichotomum (B̈rid), $-a$, feuille caulinaire $\times 24$. $b$, sommet de la feuille $\times$ i 70 . $c$, fragment d'oreillet:e. $d d$ ', margo hyalin et cellules marginales $\times 300$ - -2 . Leucoloma scopareolum C. Müll. $-a$, feuille caulinaire $\times 24 . b$, sommet de la feuille $\times 1$;o. $c$, oreillette $\times 170 . d$, margo hyalin et cellules marginales $\times 300,-3$. Leucoloma procerum Ren.Coupe transversale d'une feuille vers son milieu $\times 525 .-4$. Leucoloma Comora Ren. - Coupe transversale d'une feuille vers son milieu $\times 525$. 5. L. subchrysobasilare C. Müll. - Coupe transversale d'une feuille vers son milieu $\times 525 .-6$. L. Mafatense Ren. - Coupe transversale d'une feuille vers son milieu $\times 525$.

Pl. 22. - I. Leucoloma Crepini R. C. - Coupe d'une feuille $\times 525 .-$ 2. L. Ambreanum R. C. - Coupe d'une feuille $\times 525$. - 3. L. albocinctum R. C. - Coupe d'une feuille $\times 525$. -4 . L. Rutenbergii C. Müll. Coupe d'une feuille $\times 525$. -5 . L. capillifolium Ren. - Coupe d'une feuille $\times 525$. -6 . L. dichelymoides C. M. - Coupe d'une feuille $\times 525$. $a$, vers $1 / 6$ inférieur. $b$, vers la base de la subule. -7 . Campylopus subcomatus Ren. et Card. - $a a^{\prime}$, feuille caulinaire $\times 24 \cdot b b^{\prime}$, sommet de la feuille $\times$ I 70. $c$, oreillette $\times$ i $70 . d$, coupe de la nervure $\times 300$. $e$, cellules moyennes $\times 300$.

P1. 23. - Campylopus comatus Ren. et Card. - $a a^{\prime}$, feuille caulinaire $\times$ 24. $b b^{\prime}$, sommet de la feuille $\times 1$ jo. $c$, oreillette et tissu basilaire $\times 170 . d$, tissu vers le $\mathrm{I} / 4$ inférieur de la feuille $\times 300$. $e$, milieu de la subule $\times 300$. $f$, coupe de la nervure $\times 300$. 
Pl. 24. - Camprlopus Heribaudi Ren, et Card. - a, feuille caulinaire $\times 24 . b$, sommet de la feuille $\times 170 . c$, oreillette $\times 170 . d$, tissu basilaire $\times$ $300 . e$, tissu vers la partie supérieure de la base $\times 300 . f$, subule vers le milieu $\times$ 170. $h$, coupe de la nervure $\times 300 . g$, capsule humide $\times 24 \cdot g^{\prime}$, capsule sèche $\times 24 . i$, dent du péristome $\times 170 . k$, fragment de dent $\times 300 . l$, opercule et coiffe $\times 300 . m$, spores $\times 300$.

Pl. 25. - 1. Camprlopus rigens Ren. et Card. - a a', feuille caulinaire $\times 24 . b$, sommet de la feuille $X$ I $70 . c$, oreillette $\times$ i $70 . d$, tissu suprabasilaire $\times 300$. $e$, tissu vers $\mathrm{I} / 3$ supérieur $\times 300 . f$, tissu vers le milieu de la subule $\times 300 . \mathrm{g}$, coupe de la nervure $\times 300$. -2 . C. subvirescens Ren. et Card. $a a^{\prime}$, feuille caulinaire $\times 24 . b b^{\prime}$, sommet de la feuille $\times$ r $70 . c$, oreillette $\times$ I $70 . d$, tissu vers le milieu de la feuille $\times 300, e$, coupe de la nervure $\times 300$. - 3. C. virescens Besch. - Coupe de la nervure $\times 300$.

Pl. 26. - Campylopus filescens Ren, et Card. - $a a^{\prime} a^{\prime \prime}$, feuille caulinaire $\times 24 . b b^{\prime}$, sommet de la feuille $\times$ r 7o. $c c^{\prime}$, oreillettes $\times 170 . d$, tissu basilaire $\times 300$. e tissu vers le sommet de la feuille $\times 300: f$, capsule sèche $\times 24 . g$, dent du péristome $\times 170 . h h^{\prime}$, petites feuilles des jets filiformes $\times$ 24. $k$, coupe d'une feuille.

Pl. 27. - Camp. Flageyi Ren. Card. - $a a^{\prime} a^{\prime \prime}$, feuille caulinaire $\times 24$. $b b^{\prime}$, sommet de la feuille $\times$ i $70 . c$, oreillette $\times 170 . d$, tissu suprabasilaire $\times$ 300. $e$, cellules basilaires sinueuses $\times 300 . f$, tissu vers la base de la subule $\times$ $300 . g$, capsule $\times 24 . h$, dent du péristome $\times 170 . i$, fragment d'une dent $\times$ 300.

P1. 28. - 1. Camp. Cambouei Ren. et Card. - $a a^{\prime}$, feuille caulinaire $\times$ 24. $b b^{\prime}$, sommet de la feuille $170 . c c^{\prime}$, oreillette et tissu basilaire $\times 170 . d$, celluies juxtacostales moyennes $\times 300$. $e$, coupe de la nervure $\times 440$. Camp. laxobasis Ren. et Card. - $a a^{\prime} a^{\prime \prime}$, feuille caulinaire $\times 24 . b b^{\prime}$, sommet de la feuille $X$ i $70, c$, oreillette $X$ i $70, d$, cellules suprabasilaires $\times 500$. $e$, cellules vers $I / 3$ inférieur $\times 300 . f$, cellules vers la base de la subule $\times 300$. $g$, coupe de la nervure $\times 3$ oo.

P1. 29. - Campylopus madecassus Besch. - $a a^{\prime}$, feuille caulinaire $\times 24$. $b b^{\prime}$, sommet de la feuille $\times 170 . c$, tissu basilaire $\times$ i $70 . e$, tissu vers le milieu $\times 300$. $d d^{\prime}$, capsule $\times 24 . f$, coupe de la nervure $\times 300$.

Pl. 3o. - I. Camp. chryseolus C. Müll. $-a a^{\prime}$, feuille caulinaire $\times 24$. $b$, sommet de la feuille $\times$ i 70 . $c$, tissu suprabasilaire $\times 300$. $d$, cellules moyennes juxtacostales $\times 300 .-2$. Campylopus Caillea Ren. et Card. $a a^{\prime}$, feuille caulinaire $\times 24, b$, sommet de la feuille $\times$ i $70 . c c^{\prime}$, oreillettes $\times$ I 70. $d$, coupe de la nervure $\times 440 . e$, cellules au-dessous du milieu $\times 300 . f$, cellules au-dessus du milieu $\times 300$.

Pl. 31. - Campylopus Arbogasti Ren, et Card. - a a', feuille caulinaire $\times 24 . b$, sommet de la feuille $\times 170, c$, tissu basilaire $\times 170 . d$, tissu vers $1 / 4$ 
inférieur $\times 300 . e$, tissu sous le sommet $\times 300 . f$, coupe de la nervure $\times 440$.

Pl. 32. - Campylopus calvus Ren. et Card. - $a a^{\prime}$, feuille caulinaire $\times$ 24. $b b^{\prime}$, sommet de la feuille $\times 170 . c$, oreillette $\times 170 . d$, tissu vers le milieu de la base $\times 300$. $e$, tissu vers le sommet de la base $\times 300 . f$, coupe de la nervure $\times 300 .-2$. Campylopus hispidus Ren. et Card. - $a$ ' feuille caulinaire $\times 24 . b b^{\prime}$, sommet de la feuille $\times$ i 7o. $c c^{\prime}$, oreillette $\times 170 . d$, tissu suprabasilaire $\times 300 . e$, coupe de la nervure $\times 440$.

Pl. 33. - I. Campylopus deciduus Ren. Card. - $a$ a', feuille caulinaire $\times$ 24. $b b^{\prime}$, sommet de la feuille $\times 170 . c$, tissu basilaire $\times 170 . d$, tissu au-dessus de la base $\times 300$. $e$, coupe de la nervure $\times 550$. $e^{\prime}$, coupe de la nervure $\times 300$. 2. Campylopus polytrichoides var. altecristatus R. C. - $a$, feuille caulinaire $\times 24 . b$, sommet de la feuille $\times 170 . c$, base de la subule $\times 170 . e$, tissu basilaire $\times$ i $70 . f$, cellules moyennes juxtacostales $\times 300 . g$, coupe de la nervure $\times 300 . h$, coupe de la nervure du C. polytrichoides d'Europe $\times 300$.

Pl. 34. - 1. Campylopus flaccidus Ren. Card. - $a$ a', feuille caulinaire $\times 24 . b b^{\prime}$, sommet de la feuille $\times 170 . c$, oreillette $\times$ I $70 . d$, tissu basilaire juxtacostal $\times$ i $70 . e$, cellules suprabasilaires sinueuses $\times 300$. $f$, cellules supérieures $\times 300 . g$, coupe de la nervure dans le haut de la feuille $\times 210 . g^{\prime}$, coupe de la nervure dans la moitié inférieure $\times 87 .-2$. Camprlopus dicranelloides Ren. Card. - $a a^{\prime}$, feuille caulinaire $\times 24 . b$, sommet de la feuille $\times$ r $70 . c$, tissu basilaire $\times 170, d$, tissu de la feuille vers le milieu $\times 300 . e$, tissu de la feuille à la partie supérieure $\times 300 . f$, coupe de la nervure $\times 440$.

Pl. 35. - I. Fissidens Comorensis var. sordidus R. C. - $a$, feuille caulinaire $\times 40 . a^{\prime}$, feuille caulinaire $\times 80 . b$, tissu de la base de la lame vraie $\times$ 30o. $d$, sommet de la feuille $X$ i $70 .-2$. Fissidens Boivini var. madagassus R. C. $-a a^{\prime}$ feuille caulinaire $\times 24 . b$, sommet de la feuille $\times$ i $70 . c$, cellules moyennes de la lame vraie $\times 300$. $d$, cellules moyennes de la lame dorsale $\times 300$. $e$, cellules basilaires de la lame vraie $\times 300 .-3$. Fissidens $A r b o-$ gasti Ren. Card. - $a$, feuille caulinaire $\times 40 . b b$, sommet de la feuille $\times$ 170. $c$, cellules de lame dorsale dans le haut $\times 300$. $d$, cellules basilaires juxtacostales de la lame vraie $\times 300 .-4$. Fissidens exasperatus Ren. Card. - $a$, feuille caulinaire $\times 40$. $a a^{\prime}$, feuille caulinaire $\times 80$. $b$, sommet de la feuille $\times 300, c$, tissu à la partie supérieure de la lame dorsale $\times 300$. $d$, tissu vers la base de la lame vraie $\times 300$. e, cellules basilaires juxtacostales de la lame dorsale $\times 300 . f$, capsule $\times 24 .-5$. Fissidens hymenodon Besch. $a a^{\prime}$, feuille caulinaire $\times 40$. $b$, sommet de la feuille $\times 170$. $c$, tissu marginal basilaire de la lame vraie $\times 300, d$, tissu vers les $3 / 4$ supérieurs de la lame dorsale $\times 300 . e$, portion de la nervure $\times 170 .-6$. Fissidens ligulinus C. M. $-a a^{\prime}$, feuille caulinaire $\times 40 . b$, sommet de la feuille $\times 170 . c$, base de la lame dorsale $\times 300 . d$, tissu vers les $2 / 3$ supérieurs de la lame dorsale $\times 300$. e, portion de la nervure $\times 170$. 
P1. 36. - I. Fissidens vulcanicus Ren. Card. - $a$, feuille caulinaire $\times 40$. $b$, sommet de la feuille $\times$ i 70. $c$, groupe de cellules $\times 500 .-2$. Fissidens platyneuros Ren. Card. - $a$, feuille caulinaire $\times 80 .-b$, sommet de la feuille $\times$ r $70 . \mathcal{c}$, groupe de cellules $\times 500$. -3 . Fissidens grandiretis Ren. Card. $-a$, feuille caulinaire $\times 40, a^{\prime}$, feuille caulinaire $\times 80 . b$, sommet de feuille $\times$ r $70 . c$, base de la feuille $\times$ r $70 . d$, cellules de la lame dorsale supérieure $\times 300 .-4$. Fissidens ferrugineus $\mathrm{Hpe},-a a^{\prime} a^{\prime \prime}$, feuille caulinaire $\times 40 . b$, sommet de la feuille $\times \mathrm{r} 70 . b^{\prime}$, sommet de la feuille $\times 300$. $c$, margo de la lame vraie supérieurement $\times 300$. $c$ ', margo de la lame vraie inférieurement $\times 300 . d$, cellules supérieures $\times 300 . e$, capsule $\times 24 . f$, dent du péristome $\times 300$. - 5. Fissidens nossianus Besch. - $a a^{\prime}$, feuille caulinaire $\times$ 40. $a$ " feuille caulinaire $\times 80 . b$, cellules supérieures $\times 300 .-6$. Fissidens Motelayi, Ren. Card. $-a$, feuille caulinaire $\times 40, a^{\prime}$, feuille caulinaire $\times 80$. $b$, sommet de la feuille $\times$ i 70 . $c$, margo de la lame vraie supérieurement $\times$ $300 . c^{\prime}$, margo de la lame vraie vers la base $\times 300$. $d$,groupe de cellules $\times 300$. $e$, base de la nervure et décurrence de la lame dorsale $\times 170$.

Pl. 37. - r. Fissidens luridus Ren. Card. - a $a$, feuille caulinaire $\times$ 40. $b$, sommet de la feuille $\times 170 . b^{\prime}$, sommet de la feuille $\times 300 . c$, margo de la lame vraie $\times 300 . d$, margo et tissu de la lame dorsale $\times 300 .-2$. Conomitrium scleromitrium Besch. $-a$, feuille caulinaire $\times 40$. $b$, sommet de la feuille $\times 300$. $c$, cellules basilaires juxtacostales de la lame vraie $\times 300 . d$, margo et tissu à la base de la lame vraie $\times 300$. e, cellules marginales de la lame dorsale $\times 300 . f$, capsule $\times 24 . g$, orifice de la capsule $\times$ i $70 . g^{\prime} g^{\prime \prime}$, orifice de la capsule $\times 300$. - 3. Leucobryum Perroti Ren. Card. - $a$, feuille caulinaire $\times$ io. $b$, sommet de ia feuille $\times 8$ o. $c$, cellules moyennes $\times$ i $70, d$, cellules basilaires marginales $\times$ i $70 . e e^{\prime}$, coupe de la feuille $\times 300$.

Pl. 38. - I. Leucobryum molle C. M. - a, feuille caulinaire $\times 10$ (Sainte-Marie). $a, i d . \times$ Io (Mahambo). $b$, sommet de la feuille $\times 80$ (SainteMarie). $b$, $i d, \times 80$ (Mahambo). $c$, cellules basilaires marginales $\times$ I 70 (SainteMarie). $c^{\prime}, i d . \times 170$ (Mahambo). $d$, cellules moyennes $\times$ i 70 (Sainte-Marie). $d^{\prime}, i d . \times 170$ (Mahambo). $e$, coupe de la feuille $\times 300$ (Sainte-Marie). $e^{\prime}, i d$. $\times 300$ (Mahambo). - Lencobryum madagassum Besch. $a a^{\prime}$, feuille caulinaire $\times$ 10. $b$, sommet de la feuille $\times 8$ o. c. cellules marginales vers le milieu $\times$ 1 $70 . d$, cellules suprabasilaires marginales $\times 170 . c$, cellules moyennes $\times$ 170.

Pl. 39. - I. Leucobryum madagassum Besch. - a a', coupe de la feuille $\times 300 . b$, cellules basilaires marginales $\times 170 .-2$. Leucobryum heterodictyon Besch. - $a$, feuille caulinaire $\times$ ro. $b$, sommet de la feuille $\times 8$ o. $c$, cellules basilaires marginales $\times 170 . d$, cellules moyennes $\times 170 . e$, coupe de la feuille $\times 300$.

PI. 40. - I. Leucobryum Hildebrandtii C. M. - $a a^{\prime}$, feuille caulinaire 
$\times$ ro b. sommet de la feuille. $c$, cellules marginales vers le milieu de la feuille $\times$ i $70 . d$, cellules moyennes $\times x$ 70. $e e^{\prime}$, coupe de la feuille $\times 300 . f$, capsule $\times 24 . g g^{\prime}$, dent du péristome $\times 170 . h$, spores $\times 300$.

P1. 41. - 1. Hypophila Potierii Besch. $-a a^{\prime}$, feuille caulinaire $\times 24 . b$, cellules marginales supérieures $\times 300$. $c$, cellules supérieure $\times 300 .-2$. Hypophila lanceolata Ren. Card. - $a a^{\prime}$, feuille caulinaire $\times 24, b$, sommet de la feuille $\times$ i $70 . c$, tissu basilaire $\times 170 . d$, cellules suprabasilaires marginales $\times 300$. $e$, cellules marginales supérieures $\times 300$. -3 . Hyophila subplicata Ren. Card. $-a a^{\prime}$, feuille caulinaire $\times 24$. $b$, sommet de la feuille $\times$ 170. $c$, tissu basilaire $\times 170 . d$, cellules supérieures $\times 300$. $e$, capsule $\times 24$. $f$, fragment du péristome $\times 170$. - Hyophila Dorrii Ren. Card. - a $a^{\prime} a^{\prime \prime}$, feuille caulinaire $\times 40 . b b^{\prime} b^{\prime \prime}$, sommet de la feuille $\times$ r $70 . c$, tissu basilaire $\times$ I70. $d$, cellules supérieures $\times 300$.

Pl. 42. - I. Hyophila clavicostata Ren. Card. - a a' $a^{\prime \prime} a^{\prime \prime}$, feuille caulinaire $\times 40 . b$, sommet de la feuille $\times 170 .-2$. Pottia apiculata Kiaer. $a a^{\prime} a^{\prime \prime}$, feuille caulinaire $\times 40 . b b^{\prime}$, sommet de la feuille $\times$ i $70 . c$, tissu basilaire $\times 170 . d$, cellules basilaires juxtacostales $\times 300$. $e$, cellules supérieures $\times 300 . f f$ ', fragment de péristome. $g$, spores $\times 300$. -3 . Barbula madagassa Ren. Card. $a a^{\prime}$, feuille caulinaire $\times 40 . b$, sommet de la feuille $\times 170$. $b$, sommet de la feuille $\times 300$. $c$, cellules basilaires juxtacostales $\times 300$. $d$, tissu de la partie supérieure $\times 300$. - 4. Barbula corticicolla. - a a' feuille caulinaire $\times 24$. $b$, sommet de la feuille $\times 170$. -5 . Barbula subrevoluta Hpe. $-a a^{\prime}$, feuille caulinaire $\times 40$.

Pl. 43. - I Calymperes Isleanum Besch. $-a a^{\prime}$, feuille caulinaire $\times 24$. $b b^{\prime}$ ' sommet de la feuille $\times 80 . c$, sommet de la gaine $\times 170, d$, cellules marginales de la gaine $\times 300$. e, cellules chloropkylleuses $\times 300$. -2 . Calymperes Sancta Maria, Besch. - $a a^{\prime} a^{\prime \prime}$, feuille caulinaire $\times 24 . b b^{\prime}$, sommet de la feuille $\times 80$. c, cellules marginales de la gaine $\times$ r $70 . d$, sommet de la gaine $\times 170$.

Pl. 44. - I. Calymperes Mariei Besch. - $a a^{\prime} a^{\prime \prime}$, feuille caulinaire $\times 24$. $b b^{\prime}$, sommet de la feuille $\times 80 . c$, sommet de la gaine $\times 170 . d$, cancellines juxtacostales $\times$ i $70 . e$, cellules marginales moyennes de la gaine $\times 300$. 2. Calymperes Nossi Comba Besch. $-a a^{\prime}$, feuille caulinaire $\times 24 . b b^{\prime}$, sommet de la feuille $\times 8$ o. $c$, sommet de la gaine $\times$ r $70 . d$, marges du sommet de Ia gaine $\times 300$. e, marges de la base de la gaine $\times 300$.

Pl. 45. - x. Calymperes crassilimbatum Ren. Card. - a $a^{\prime} a^{\prime \prime}$, feuille caulinaire. $b b^{\prime}$, sommet de la feuille $\times 80, c$. base de la feuille $\times 80 . d d$, sommet de la gaine $\times 170 . e$, marge ventrale $\times 300 . f$, marge basilaire $\times 300$.

P1. 46. - Calymperes Polii Besch. - $a a^{\prime}$, feuille caulinaire $<24 . b^{\prime}$, sommet de la feuille $\times 80$, $b^{\prime}$, sommet de la feuille $\times 300$. c, sommet de la 
gaine $\times$ i $70 . d$, marges un peu au-dessus de la gaine $\times 3$ oo. $e$, marges vers le milieu de la gaine $\times 300 . f$, marges à la base de la gaine. $g$, cancellines juxtacostales $\times 170 . h$, capsule $\times 24 . i$, spores $\times 300$.

Pl. 47. - Calymperes hispidum Ren. Card. - $a a^{\prime} a^{\prime \prime} a^{\prime \prime}$, feuille caulinaire $\times 24 . b b^{\prime} b^{\prime \prime}$, sommet de la feuille $\times 80 . c$, gaine de la feuille $\times 80 . d$, sommet de la gaine $\times 1$; o. $e$, téniole ventrale. $f$, téniole basilaire $\times 300 . g$, cancellines juxtacostales $\times 170 . h h$, cellules chlorophylleuses $\times 300 . i$, capsule $\times 24 . j j$, opercule $\times 40, k$, spores $\times 300$.

Pl. 48. - I. Calymperes reduncum Kiaer. $-a a^{\prime}$, feuille caulinaire $\times 24$. $b b^{\prime} b^{\prime \prime}$, sommet de la feuille $\times 80 . c$, sommet de la gaine $X$ i 7o. $d$, téniole ventrale $\times 300, e$, téniole basilaire $\times 300 . f$, cellules chlorophylleuses $\times 300$. - 2. Calymperes decolorans C. Müll. $-a$, feuille caulinaire $\times 24$. $b$. sommet de la feuille $\times 80, b^{\prime}$, sommet de la feuille $\times$ i $70, c$, sommet de la gaine $\times$ i $70 . f$, téniole basilaire $\times 300 . e$, cancellines juxtacostales $\times \mathbf{r} 70 . f$, cellules chlorophylleuses $\times 300 . g$. capsule $\times 24$.

P1. 49. - I. Syrrhopodon Nossibeanus Besch. var. borbonicus, Ren. Card. $-a$, feuille caulinaire $\times 24 . \quad b$. sommet de la feuille $\times 80$. $c$. portion de la lame chlorophylleuse supérieure $\times 80 . d$, gaine de la feuille $\times 40$. $e$, margo intramarginal et cellules marginales vers la base $\times 300$. $f$, margo et cellules marginales vers le milieu de la gaine $\times 300$. $g$, limbe et cellules marginales vers la partie supérieure de la gaine $\times 300$. $h$, sommet de la gaine $\times$ ro7. $i$, cellules chlorophylleuses supérieures $\times 300$.

Pl. 50. - 1. Syrrhopodon Chenagoni Ren. Card. - $a a^{\prime} a^{\prime \prime} a^{\prime \prime}$, feuille caulinaire $\times 24, b$, feuille caulinaire $\times 40 . c c^{\prime}$, sommet de la feuille $\times 170$. $d$, sommet de la gaine $\times 300 . e$, marges basilaires $\times 300 . f$, portion de la lame chlorophylleuse au-dessus de la gaine $\times 300 . g$, portion de la lame chlorophylleuse vers le milieu de la feuille $\times 300 . h$, cellules chlorophylleuses supérieures $\times 300$. $i$ cellules chlorophylleuses marginales $\times 300$.

Pl. 51. - I. Syrrhopodon microbolacus C. Müll. - $a$, feuille caulinaire $\times 24 . a^{\prime}$, feuille caulinaire $\times 40 . b b^{\prime}$, sommet de ia feuille $\times$ r $70 . c$, base de la feuille $\times$ izo. $d$, margo basilaire $\times 300$. $e$, margo moyen $\times 300 . f$, cellules chlorophylleuses $\times 300 . g$, capsule $\times 24$. $h$, portion du péristome $\times 300 . j$, spores $\times 300 .-2$. Syrrhopodon hispidocostatus Ren. Card. - $a a^{\prime} a^{\prime \prime}$, feuille caulinaire $\times 24 . b b^{\prime}$. sommet de la feuille $\times$ r $70, c$, sommet de la gaine. $\times 170 . d$, portion de la lame chlorophylleuse moyenne $\times 300 . e$, base de la feuille $\times$ i 7o. $f$, portion de la lame chlorophylleuse supérieure $\times 300$.

Pl. 52. - I. Syrrhopodon sparsus Ren. Card. - $a a^{\prime}$, feuille caulinaire $\times 24 . b b^{\prime}$, sommet de la feuille $\times 80$. $c$, gaine de la feuille $\times 80 . d$, margo et cancellines basilaires $\times 300$. e, sommet de la gaine $\times$ I $70 . f$, marge au sommet de la gaine. $g$, marge au-dessous du sommet de la gaine $\times 300 . h$, 
cellules chlorophylleuses inférieures $\times 300 . i$, cellules chlorophylleuses supérieures $\times 300 .-2$. Syrrhopodon graminifolius Ren. Card. $-a a^{\prime}$, feuille caulinaire. $b$, sommet de la feuille $\times 80 . b^{\prime}$, sommet de la feuille $\times$ i $70 . c$, gaine de la feuille $\times 80$. $d$, sommet de la gaine $\times 170 . e$, marge moyenne de la gaine $\times 300 . f$, cellules chlorophylleuses $\times 300$.

P1. 53. - I. Syrrhopodon Lepervanchei Besch. - a a', feuille caulinaire $\times 24 . b$, sommet de la feuille $\times 170, b$, sommet de la feuille $\times 300$. $c$, sommet de la gaine $\times 170 . d$, marge basilaire $\times 300 . e$, marge du sommet de la gaine $\times 300 .-1$. Syrrhopodon glaucophyllus Ren. Card. $-a a^{\prime}$, feuille caulinaire $\times 24 . b$, sommet de la feuille $\times 170 . c$, sommet de la gaine $\times 170$. - 3. Syrrhopodon glaucophrllus, var, rufus Ren. Card. - a $a^{\prime} a^{\prime \prime}$, feuille caulinaire $\times 24 . b$, gaine de la feuille $\times 80 . c$, sommet de la gaine $\times$ i 70 .

Pl. 54. 1. - Syrrhopodon spiralis Ren. Card. - $a a^{\prime}$, feuille caulinaire $\times 24 . b b^{\prime}$, sommet de la feuille $\times 170, c$, gaine de la feuille $\times 80 . d$, sommet de la gaine $\times$ i 70 . $e$, marge de la lame chlorophylleuse $\times 300$. $f$. cellules chlorophylleuses $\times 300$. - 2. Syrrhopodon subflavus Ren. Card. $-a a^{\prime}$ feuille caulinaire $\times 24 . b b^{\prime}$, sommet de la feuille $\times 80, c$, base de la feuille $\times$ 170. $d d^{\prime}$, sommet de la gaine $\times 370 . e$, cellules chlorophylleuses $\times 300$.

P1. 55. - 1. Coleochatium plicatum (P. B.) - $a$ a', feuille de la tige primaire $\times 40 . b^{\prime} b^{\prime} b^{\prime \prime}$, feuille raméale $\times 40 . c$, base de la feuille $\times 300 .-2$. Coleochatium appendiculaţum Ren. Card. - $a a^{\prime}$, feuille de la tige primaire $\times 40, b b^{\prime} b^{\prime \prime}$, feuille raméale $\times 40 . c c^{\prime}$, sommet de la feuille $\times$ i $70 . d d^{\prime}$, angles basilaires de la feuille $\times 300 . e$, cellules supérieures $\times 300 .-3 . D a$ simitrium borbonicum Besch. - $a a^{\prime}$, feuille de la tige primaire $\times 40 . b b^{\prime}$, feuille raméale $\times 40, c$, sommet de la feuille $\times$ i $70 . d$, cellules supérieures $\times$ 300. $e$, cellules basilaires $\times 300 . f$, foliole périchétiale $\times 40 . g$, fragment de péristome $\times$ I 70 .

Pl. 56. - I. Schlotheimia squarrosa Brid. - a $a^{\prime} a^{\prime \prime} a^{\prime \prime}$, feuille raméale $\times 40 . b$, sommet de la feuille $\times$ i 70. $c$, cellules basilaires $\times 300$. $d$, cellules supérieures $\times 300 . f$, capsule $\times 24 . g g^{\prime}$, coiffe $\times 24 . h$, fragment du péristome $\times 170 . i$, spores $\times 300,-3$. Schlotheimia microphylla Besch. $-a$ a, feuille raméale $\times 40 .-3$. Schlotheimia microcarpa Sch. - a a' a" $a^{\prime \prime \prime}$, feuille raméale $\times 40 .-4$. Schlotheimia trichophora Ren. Card. $-a z^{\prime} a^{\prime \prime}$, feuille de la tige primaire $\times 40 . b b^{\prime} b^{\prime \prime} b^{\prime \prime \prime}$, feuille raméale $\times 40 . c c^{\prime}$, sommet des feuilles raméales $X 170$.

Pl. 57. - I. Schlotheimia Boivini C. Müll. - $a a^{\prime} a^{\prime \prime} a^{\prime \prime \prime}$, feuille raméale $\times 40$. $b$, cellules basilaires $\times 300 . c$, cellules moyennes juxtacostales $\times 300$. $d$, cellules supérieures $\times 300$. $e$, capsule $\times 24 \cdot f$, coiffe $\times 24 \cdot f^{\prime}$, sommet de la coiffe $\times 80 . g$, fragment du péristome $\times 170 . h$, spores $\times 300,-2$. Schlotheimia linealis $\mathrm{C}$. Müll. $-a a^{\prime} a^{\prime \prime}$, feuille raméale $\times 40$. 
Pl. 58. - I. Schlotheimia badiella Besch. - $a a^{\prime} a^{\prime \prime} a^{\prime \prime \prime, ~ f e u i l l e ~ r a m e ́ a l e ~}$ $\times 40 . b$, sommet de la feuille $\times 80, b^{\prime}$ sommet de la feuille $\times 170$. $c$, cellules supérieures $\times 300 . c^{\prime}$, cellules supérieures $\times 550$. $d$, capsule $\times 24$. $e$, coiffe $\times 24$. $e^{\prime}$, sommet de la coiffe $\times 80 . f$, fragment du péristome $\times$ i $70 . g$, spores $\times 300 .-2$. Schlotheimia trypanoclada C. Müll. - $a a^{\prime} a^{\prime \prime}$, feuille raméale $\times 40$.

Pl. 59. - Schlotheimia phacochlora Besch. - $a a^{\prime} a^{\prime \prime}$, feuille raméale $\times$ $b b^{\prime}$, cellules basilaires $\times 300 . c c^{\prime}$, cellules moyennes $\times 300 . d d^{\prime}$, cellules supérieures $\times 300 . e$, coiffe $\times 24 . e^{\prime}$, sommet de la coiffe $\times 80 . f f^{\prime}$, capsule $\times$ 24. $g g^{\prime}$, fragment du péristome $\times$ i $70 . h h^{\prime}$, spores $\times 300$.

Pl. 6o. - I. Schlotheimia Perroti Ren. Card. - $a$, feuille de la tige primaire $\times 40 . b b^{\prime} b^{\prime \prime} b^{\prime \prime \prime}$, feuille raméale $\times 40 . c$, sommet de la feuille $\times 80 . d$, foliole périchétiale $\times 40$. $e$, cellules basilaires $\times 300 . f$, cellules supérieures $\times 300 . g$, capsule $\times 24$. $h$, coiffe, $h$, sommet de la coiffe $\times 80$. $i i$, dents du péristome $\times$ i $70 . k$, spores $\times 300 .-2$. Schlotheimia Nossibeana Besch. $a a^{\prime} a^{\prime \prime}$, feuille raméale $\times 4^{\circ}$.

Pl. 61. - I. Schlotheimia conica Ren. et Card. - $a a^{\prime}$, feuille raméale $\times$ 40. $b$, cellules basilaires $\times 300$. $c$, cellules supérieures $\times 300$. $d$, capsule $\times 24 . e e^{\prime}$, fragment du péristome $\times$ i $70 . f$, coiffe $\times 24 . f^{\prime}$, sommet de la coiffe $\times 80 . g$, spores $\times 300 .-2$. Schlotheimia tenuiseta C. Müll. $-a a^{\prime} a^{\prime \prime}$, feuille raméale $\times 40 . \bar{b}$, cellules basilaires $\times 300$. $c c^{\prime}$, cellules marginales moyennes $\times 300$.

Pl. 62. I. Schlotheima foveolata Ren. et Card. $-a a^{\prime}$, feuille raméale. $\bar{b}$, sommet de la feuille $\times 80 . c c^{\prime}$, cellules basilaires $\times 300$. $d$, cellules moyennes $\times 300$. $e$, cellules supérieures $\times 300$. - Physcomitrium dilatatum Ren. et Card. $-a$, feuille caulinaire $\times 24 . b$, sommet de la feuille $\times 170$. c, marges suprabasilaires de la feuille $\times$ i $70 . d$, marges médianes de la feuille $\times$ i 70 , $e e^{\prime} e^{\prime \prime}$, capsule $\times 24 . f$, membrane capsulaire (exothecium) $\times 170 . g$, opercule $\times 24$. $h$, cellules de l'opercule $\times 170 . i$, spores $\times 300$.

Pl. 63. - I. Brachymenium Borgeni Hpe. $-a a^{\prime}$, feuille caulinaire $\times$ 40. $b$, sommet de la feuille $\times 170$. $c$, cellules moyennes juxtacostales $\times 170$. $d$, tissu basilaire $\times 170$. $c$, cellules moyennes juxtacostales $\times 170$. $d$, tissu basilaire $\times$ i $70 . e$, capsule $\times 24 . f$, fragment de péristome $\times$ i $70 . g$, spores $\times 300 .-2$. Brachymenium madagassum $\mathrm{Hpe}$ - - $a a^{\prime}$, feuille caulinaire $\times$ 40. $\quad b b^{\prime}$, sommet de la feuille $\times 170$. $c$, tissu basilaire $\times 170$. $d$, cellules moyennes juxtacostales $\times$ i 70. e. $e^{\prime}$, capsule $\times 24 . f$, spores $\times 300 .-3$. Brachymenium Heribaudi Ren. Card. $-a a^{\prime}$, feuille caulinaire $\times 40 . b$, sommet de la feuille $\times 170$. c, cellules moyennes $\times$ i $70 . d$, tissu basilaire $x^{\prime}$ 170. $e$, capsule $\times 24 . f$, spores $\times 300$.

PI. 64. - I. Brachymenium Philonotula Hpe. - a a' feuille caulinaire 
$\times 40, b$, sommet de la feuille $\times 170 . c$, cellules moyennes $\times 170 . d$, tissu basilaire $\times 170 . e e^{\prime}$, capsule $\times 24 . f$, dent externe du péristome $\times 170 . g$, spores $\times 300 .-2$. Brachymenium capitulatum Mitt. - $a$, feuille caulinaire $\times$ 40. $b$, sommet de la feuille $\times 170$. $c c$, celluies marginales moyennes $\times$ I 70. $d$, capsule $\times 24, e$, dent externe du péristome $\times$ i $70 . f$, spores $\times 300$. - 3. Brachymenium nigrescens Besch. - $a$, feuille caulinaire $\times 24 . b$, sommet de la feuille $\times 170 . c$, cellules marginales moyennes $\times 170$.

Pl. 65. - Cryphra subintegra R. C. - $a$, feuille caulinaire $\times 40 . b b^{\prime}$, sommet de la feuille $\times 300 . c$, cellules médianes juxtacostales $\times 300 . d$, foliole périchétiale $\times 40 . e$, sommet d'une foliole périchétiale $\times 30 . f$ cellules suprabasilaires d'une foliole périchétiale $\times 300 . g g^{\prime}$, capsule $\times 24 . h$, opercule avec fragment de l'anneau $\times 40 . i$, coiffe $\times 40 . j$, dents péristomiales $\times 170$. $k$, dent péristomiale et anneau $\times \mathbf{i} 7^{\circ} . k^{\prime}$, fragment d'anneau $\times 300 . l l^{\prime}$, spores $\times 300$.

Pl. 66. - 1. Pterogoniella Sancta Maria Besch. - $a a^{\prime} a^{\prime \prime}$, feuille caulinaire $\times 40 . b$, sommet de la feuille $\times 170 . c$, cellules alaires $\times 300 . e$, cellules moyennes $\times 300 . e$, cellules subapicales $\times 300 . ~ f f^{\prime} f^{\prime \prime}$, capsule $\times 24 . g$, fragment du péristome et de l'exothecium $\times 170 . h$, cellules de l'exothecium $\times$ 30o. $i$, spores $\times 300$. -2 . Pterogoniella madagascariensis Brid. $a a^{\prime} a^{\prime \prime} a^{\prime \prime}$, feuille caulinaire $\times 40 . b b^{\prime}$, sommet de la feuille $\times$ r $70 . c$, cellules alaires $\times 300 . d$, cellules basilaires de la feuille et superficielles de la tige $\times$ 3oo. $e e^{\prime}$, cellules moyennes $\times 300 . f$, cellules subapicales $\times 300$.

Pl. 67. - 1. Pterogoniella diversifolia R. C. - $a a^{\prime} a^{\prime \prime} a^{\prime \prime \prime} a^{\prime \prime \prime \prime, ~ f e u i l l e ~}$ caulinaire $\times 40$. $b$, sommet de là feuille $\times$ i 70 . c cellules des angles $\times 300$. $d$, cellules moyennes $\times 300 . e$, cellules subapicales $\times 300 . f f$, foliole périchétiale $\times 40 . g g^{\prime} g^{\prime \prime}$, capsule $\times 24$. $h$, dent du péristome et fragment d'exothecium $\times$ i 70. $i$, cellules de l'exothecium $\times 300 . j$, fleur mâle $\times 40 . k k^{\prime}$, foliole périgoniale $\times 40$. l, anthéridie $\times 170 . m$, spores $\times 300 .-2$. Pterogoniella $o b-$ tusifolia R. C. $-a a^{\prime} a^{\prime \prime}$, feuille caulinaire $\times 40$. $b$, sommet de la feuille $\times$ 170. $c c^{\prime}$, cellules des angles $\times 300$. $d$, cellules moyennes $\times 300 . e$, cellules subapicales $\times 300$.

P1. 68. - I. Leucodon Rutenbergii C. M. - a $a^{\prime} a^{\prime \prime}$, fenille caulinaire $\times$ $24 . b$, sommet de la feuille $\times 170$. c, cellules marginales supérieures $\times 300$. $d$, cellules moyennes $\times 300$. $e$, cellules des angles $\times 300 . f$, capsule. -2 . Lepyrodon mauritianus C. M. $-a a^{\prime}$, feuille caulinaire $\times 24 . b b^{\prime}$, feuille caulinaire de la var. latifolitus $\times 24 . c c^{\prime}$, sommet de la feuille $\times 170, d$, cellules supérieures $\times 300$. e, cellules moyennes $\times 300 . f$, cellules alaires $\times$ 30o. $g$, foliole périchétiale externe $\times 24 . g^{\prime} g^{\prime \prime} g^{\prime \prime}$, fol. périch. interne $\times 24$. $h h^{\prime}$, capsule $\times 24 . i i^{\prime}$, dents du péristome et fragment d'exothecium laissant voir un anneau obscur $\times 170 . j$, spores $\times 300$. 
Pl. 69. - Jagerina solitaria (Brid.). - $a a^{\prime} a^{\prime \prime}$, feuille caulinaire $\times$ 24. $b$, sommet de la feuille $\times$ r $70 . c$, cellules moyennes internes $\times 300 . d$, cellules moyennes marginales $\times 300 . e$, tissu dés angles basilaires $\times \mathbf{r} 70 . f$, toliole périchétiale $\times 24 . g$, sommet de la foliole $\times$ i $70 . h$, capsule et foliole périchétiale $\times 24 . i$, coiffe $\times 40 . j$, fragment de péristome et d'exothecium $\times$ r $70 . k$, spores $\times 300$.

Pl. 70. - Jaegerina stolonifera C. M. - $a a^{\prime}$, feuille caulinaire $\times 24 \cdot b$, sommet de la feuille $\times$ i $70 . c$, base de la feuille $\times 80$. c', cellules des angles $\times$ $300 . c$ ", cellules basilaires $\times 300 . d$, cellules moyennes juxtacostales $\times 300 . e$, cellules moyennes marginales $\times 300 ., f f^{\prime} f^{\prime \prime}$, fragment de péristome et d'exothecium $\times$ i $70 . h$, coiffe $\times 40 . i$, spores $\times 300$.

Pl.71. - I. Garovaglia patentissima (Hpe) C. M. - a a', feuille caulinaire $\times 24 . b b^{\prime}$, feuille raméale $\times 24 . c c^{\prime}$, cellules des angles basilaires $\times 170$. $d$, cellules basilaires médianes $\times 300 . e$, cellules moyennes $\times 300$. $f$, fleur femelle $\times 24 \cdot g g^{\prime}$, foliole périchétiale $\times 24 \cdot h$, archégone $\times 80 .-2$. Garovaglia Bescherellei (Kiaer) R. C. $-a a^{\prime} a^{\prime \prime} a^{\prime \prime \prime}$, feuille caulinaire $\times 24 . b$, feuille caulinaire $\times 40$. c, sommet de la feuille $\times$ i 70. $d$, base de la feuille $X$ I70. $e$, cellules moyennes $\times 300$.

Pl. 72. - I. Renauldia Hildebrandtielloides (R. C.) C. M. - $a a^{\prime} a^{\prime \prime}$, feuille caulinaire $\times 24, c$, sommet de la feuille $\times 24 . c$, cellules supérieures $\times$ $300 . d$, cellules moyennès $\times 300$. $e$, cellules basilaires médianes $\times 300 . f$, cellules des angles $\times 300 . g$, foliole périchétiale $\times 24 . h$, cellules des angles de la foliole $\times 300$. $i$, cellules moyennes de la foliole $\times 300 . j$, périchèze. $k$, capsule $\times$ ro, $l$, opercule $\times 40, m$, coiffe $\times 20, n$, péristome vu sur la face interne $\times 170.0$, fragment du péristome vu sur la face externe et de l'exothecium $\times 170 . p$, spores $\times 300$.

P1. 73. - 1. Renauldia dichotoma C. M. $-a \cdot a$, feuille caulinaire $\times 24 . b$, sommet de la feuille $\times 170 . c$, cellules moyennes juxtacostales $\times 300$. $d$, cellules moyennes marginales $\times 300$. e, cellules suprabasilaires $\times 300 . f$, base de la feuille $\times$ r $70 . g$, périchèze $\times 20 . h$, foliole périchétiale $\times 20 . i$, capsule. $j$, opercule $\times 40 . k k^{\prime}$, coiffe $\times 40 . l$, fragment de péristome et d'exothecium $\times 170 . m m^{\prime}$, spores $\times 300,-2$. Camptochate (?) fallax R. C. $-a a^{\prime} a^{\prime \prime} a^{\prime \prime \prime}$ $a^{\prime \prime \prime}$, feuille caulinaire $\times 40, b$, sommet de la feuille $\times 170 . b$, sommet de la feuille $\times 300$. $c$, cellules supérieures marginales $\times 300$. $d$, cellules moyennes $\times 300, e$, cellules des angles basilaires $\times 300$.

P1. 74. - I. Hildebrandtiella longiseta R. C. - $a$ a' $a^{\prime \prime} a^{\prime \prime}$ ' $a^{\prime \prime \prime \prime, ~ f e u i l l e ~}$ caulinaire $\times 24, b$, cellules subapicales $\times 300 . c$, cellules moyennes $\times 300 . d$, cellules des angles basilaires $\times$ i 70. $e$, cellules basilaires médianes $\times 300 . f$, capsule et coiffe $\times 24 . g$, périchèze et capsule $\times 6 . h$, fragment de péristome vu sur la face externe $\times 170$. $i$, fragment de péristome va sur la face interne 
$\times$ 170. $j$, spores $\times 300$. - 2. Hildebrandtiella cuspidans Besch. $-a a^{\prime}$, feuille caulinaire $\times 24, b$, cellules des angles $\times 170$. c , spores $\times 300 .-3$. Hildebrandtiella pachyclada Besch. - $a a^{\prime}$, feuille caulinaire $\times 24 . b b^{\prime}$, cellules subapicales $\times 300 . c c^{\prime}$, cellules moyennes $\times 300 . d$, cellules des angles $\times 170 .-4$. Hildebrandtiella rotundifolia Ren. $-a a^{\prime}$, feuille caulinaire $\times 24 . b$, cellules moyennes $\times 300 . c$, cellules des angles $\times 170$. $d$, corpuscule cloisonné porté par la plante $\times 170$.

P1. 75.- 1. Papillaria Ankaratrensis Kiaer. - a, feuille caulinaire $\times 40 . a^{\prime}$, feuille raméale $\times 40 . b$, cellules basilaires juxtacostales $\times 300, c$, cellules des angles $\times 300, d$, cellules moyennes juxtacostales $\times 300 . e$, cellules moyennes $\times 300 .-2$. Papillaria africana C. M. $-a a^{\prime}$, feuille caulinaire $\times 40$ (type du Cap). $b$, feuille caulinaire $\times 20$ (Madagascar). $b^{\prime}$, feuille raméale $\times 40$ (Madagascar). $c$, sommet de la feuille caulinaire $\times$ i $70 . d$, base de la feuille $\times 80$. - 3. Papillaria Boivini Besch. $-a$, feuille caulinaire $\times 40$ (type de Maurice). $a$, feuille raméale $\times 40$. (type de Maurice). $b$, feuille caulinaire $\times 4$ o (forme macrotis, Madagascar). $b^{\prime}$, feuille raméale $\times 40$ (forme macrotis, Madagascar). $c c$, feuille caulinaire $\times 40$ (f. gracilis, Madagascar). $c^{\prime} c^{\prime} c^{\prime}$, feuille raméale $\times$ 40 (f. gracilis, Madagascar). - 4. Papillari Boivini? - $a a^{\prime}$, feuille caulinaire $\times 40 . a^{\prime \prime}$, feuille raméale $\times 40 . b$, foliole périchétiale externe $\times 40 . b$, foliole périchétiale moyenne $\times 40 . b^{\prime \prime}$, foliole périchétiale interne $>40, c$, cellules moyennes de la foliole périchétiale $\times 300 . d$, cellules supérieures de la foliole périchétiale $\times 300$. $e$, capsule et périchèze $\times 24 . f$, paraphyse $\times 40$. $g$, cellules du sommet de la paraphyse $\times 300 . h$, cellules de la base de la paraphyse $\times 300$ (Madagascar).

P1. 76. - I. Papillaria lata R. C. $-a a^{\prime} a^{\prime \prime}$, feuille caulinaire $\times 40 . b$, sommet de la feuille $\times$ r $70 . c$, base de la feuille $\times$ r $70 . d$, cellules basilaires juxtacostales $\times 300$. $e$, cellules des angles $\times 300 . f$, cellules marginales moyennes $\times 300 .-2$. Papillaria appendiculata R.C. $-a a^{\prime} a^{\prime \prime}$, feuille caulinaire $\times$ 40. $b$, base de la feuille $\times$ i $70 . c$, cellules basilaires juxtacostales $\times$ $300 . d$, cellules des angles $\times 300 . e$. cellules moyennes juxtacostales $\times 300 . f$, cellules supérieures marginales $\times 300 .-3$. Papillaria Borchgrenvinkii Kiaer. - $a a^{\prime}$, feuille caulinaire $\times 40 . \quad b b^{\prime}$, feuille raméale $\times 40$. c, sommet de la feuille caulinaire $\times$ i $70, d$, base de la feuille $\times$ i $70 . e$, tissu des angles $\times 300$. $f$, cellules moyennes marginales $\times 300 . g$, cellules supérieures $\times 300 .-4$. Papillaria acinacifolia Besch. $-a a^{\prime}$, feuille caulinaire $\times 40$.

Pl. 77. - 1. Papillaria fulvastra Besch. - a $a^{\prime} a^{\prime \prime} c c^{\prime}$, feuille caulinaire $\times 40, c^{\prime \prime}$, feuille raméale $\times 40, b$, sommet de la feuille caulinaire $\times 80 . b^{\prime}$, sommet de la feuille caulinaire $\times 300$. $h$, cellules supérieures marginales $\times$ $300 . i$, base de la feuille $\times 300 . d d$, feuille caulinaire $\times 40$ (var. madagassa). $e$, sommet de la feuille $\times 300$ (var. madagassa). $f$, cellules supérieures marginales $\times 300$. (var. madagassa), g, cellules moyennes $\ 300$ (var. madagassa). 
- 2. Papillaria floribunda Doz. Molk. - $a a^{\prime} a^{\prime \prime} a^{\prime \prime} a^{\prime \prime \prime}$, feuille caulinaire $\times 40$ (Java,Hb. Mus., Paris). $b$, sommet de la feuille $\times 300 . c$, cellules supérieures marginales $\times 300 . d$, sommet de la feuille caulinaire $\times 80$. $e$, cellules des angles $\times 300 . f f^{\prime}$, feuille raméale $\times 40 . g g^{\prime}$ feuille caulinaire $\times 40$ (Java, Massart). $h$, sommet de la feuille $\times 80$. $h$, sommet de la feuille $\times 300$. $i$, cellules marginales moyennes $\times 300 . j$, cellules marginales suprabasilaires $\times 300$.

Pl. 78. - 1. Papillaria pseudo-fulvastra C. M. - $a a^{\prime}$, feuille caulinaire $\times 40 . b$, sommet de la feuille $\times 80$. $b^{\prime}$, sommet de la feuille $\times 300$. $c c^{\prime}$, cellules des angles $\times 300 . d$, cellules marginales moyennes $\times 300 . e e^{\prime}$, feuille caulinaire $\times 40$. (forma squarrosa). $f$, cellules moyennes $\times 300$. -2 . Trachypus serrulatus (Brid). - $a a^{\prime}$ feuille caulinaire $\times 40, b$, sommet de ia feuille $\times 170 . c$, cellules des angles $\times 300 . d$, cellules basilaires juxtacostales $\times 300$. $e$, cellules moyennes $\times 300 . f$, cellules marginales moyennes $\times 300$.

P1. 79. - 1. Pilotrichella pseudo imbricata C. M. - $a a^{\prime}$, feuille caulinaire $\times 40 . b^{\prime} b^{\prime \prime}$, feuille raméale $\times 40 . c$, sommet de la feuille caulinaire $\times 80 . d$, sommet de la feuille raméale $\times 80$. $e$, cellules des angles $\times 300$. $f$, cellules moyennes $\times 300 . g^{\prime} g^{\prime} g^{\prime \prime}$, foliole périchétiale $\times 40 . h$, capsule et périchèze $\times$ 24. $j$, fragment de péristome et d'exothecium $\times 170 . k k^{\prime}$, fragment de dent interne avec membrame basilaire $\times 300$. $l$, spores $\times 300$. -2 . Pilotrichella subimbricata $\mathrm{H}_{\text {pe. }}-a a^{\prime}$, feuille caulinaire $\times 40, b b^{\prime}$, sommet de la feuille $\times$ 8o. $c$, cellules marginales supérieures $\times 300 . d$, cellules moyennes $\times 300$. $e$, cellules des angles $\times 300, f f^{\prime}$, feuille raméale $\times 40, g$, cellules marginales supérieures $\times 300 . h$, cellules des angles $\times 300$.

PI. 8o. - I. Pilotrichella subimbricata var. Flageyi R. C. - $a a^{\prime}$, feuille caulinaire $\times 40, b$, sommet ${ }^{\circ}$ de la feuille $\times 80$. $c$, cellules marginales supérieures $\times 300 . d$, cellules moyennes $\times 300$. $e$, cellules des angles $\times 300 . f$, feuille raméale $\times 40 . g$, cellules des angles $\times 300$. $h$, foliole périchétiale et archégone $\times 80 .-2$. Pilotrichella imbricatula C. M. $-a a^{\prime} a^{\prime \prime}$ feuille caulinaire $\times 40 . b$, cellules supérieures $\times 300 . c$, cellules moyennes 300 . $d$, cellules des angles $\times 300$. e e $e^{\prime \prime} e^{\prime \prime} e^{\prime \prime}$, feuille raméale $\times 40 . f$, cellules des angles $\times 3$ oo. $g g^{\prime}$, feuille caulinaire $\times 40$ (var. costata). $h h^{\prime}$, feuille raméale $\times 40$ (var. costata).

PI. 8 I - I. Pilotrichella Grimaldii R.C. $-a a^{\prime} a^{\prime \prime}$, feuille caulinaire $\times 40$. $b$, ceilules des angles $\times 300$. c c cellules moyennes $\times 300 . d d^{\prime}$, feuille raméale $\times 40$. $e$, cellules des angles $\times 300 . f f^{\prime} f^{\prime \prime}$, folioles périchétiales $\times 40 . g g^{\prime}$ capsule $\times 24 . h$, fragment de péristome $\times 170 . i i^{\prime}$,fragment d'une dent interne avec membrane basilaire $\times 300, j$, spores $\times 300$. -2 . Pilotrichella biformis Hpe. $-a a^{\prime}$, feuille caulinaire $\times 40 . b$, cellules des angles $\times 300 . c$, cellules moyennes $\times 300 . d d^{\prime}$, feuille raméale $\times 40$. e, cellules des angles $\times 300$. 
Pl. 82. - I. Pilotrichella biformis var. congesta R. C. - a a', feuille raméale $\times 40 . c$, cellules des angles $\times 300,-2$. Pilotrichella Mascarenica $\mathrm{C}$. M. $-a a^{\prime}$, feuille caulinaire $\times 40 . b$, cellules moyennes $\times 300 . c$, cellules des angles $\times 300 . d d^{\prime}$, feuille raméale $\times 40 . e$, cellules des angles $\times 300 . f f^{\prime} f^{\prime \prime}$, foliole périchétiale $\times 24 . g$, capsule $\times 24 . h$, fragment de péristome externe $\times$ i 70 . $i$, spores $\times 300$.

Pl. 83. - 1, Pilotrichella longinervis R. C. - a a' $a^{\prime \prime} a^{\prime \prime}$, feuille caulinaire et du stipe $\times 40 . b$, cellules moyennes $\times 300$. $c$, cellules des angles $\times 300$. $d d^{\prime} d^{\prime \prime}$, feuille raméale $\times 40$. $e$, sommet de la feuille $\times 170 .-2$. Aerobryum pseudocapense C. M. - $a a^{\prime} a^{\prime \prime}$, feuille caulinaire $\times 24$. $b$, cellules marginales moyennes $\times 300 . c$, cellules moyennes $\times 300$. $a d^{\prime} d^{\prime \prime}$, feuille caulinaire de la var. majus $\times 24$. $e$, cellules moyennes $\times 300$. -3 ، Aerobryum subpiligerum Hpe. - $a a^{\prime} a^{\prime \prime}$, feuille caulinaire $\times 24$ (type de Borgen). $b$, cellules marginales moyennes $\times 300$. $c$, cellules moyennes $\times 300 . d d^{\prime} d^{\prime \prime}$, feuille caulinaire $\times 24$ (Amperifery). $e e^{\prime} e^{\prime \prime} e^{\prime \prime}$, feuille caulinaire $\times 24$ (Ampasimbé). $f f^{\prime}$, feuille caulinaire $\times 24$ (var. congestum). $g g^{\prime} g^{\prime \prime}$, foliole d'un périchèze non fructifié $\times 24 . h$, archégone et paraphyse $\times 80$.

Pl. 84. - 1. Aerobryum lanosulum C. M. $-a a^{\prime}$, feuille caulinaire $\times 24$. $b$, cellules marginales moyennes $\times 300$. c cellules moyennes $\times 300,-2$. Aerobryum capillicaule R. C. - $a a^{\prime} a^{\prime \prime}$, feuilles caulinaires et de la base des rameaux $\times 24 . b$, cellules marginales moyennes $\times 300$. $c$, cellules moyennes $\times 300 . d$, cellules des angles $\times 300 . e e^{\prime} e^{\prime \prime}$, feuilles des prolongements filiformes $\times 24 . f$, cellules marginales moyennes $\times 300$. -3 . Neckera Boivini C. M. $-a a^{\prime} a^{\prime \prime}$, feuille caulinaire $\times 24 \cdot b$, fragment du sommet de la feuille $\times 300 . c c^{\prime}$ cellules marginales moyennes $\times 300$. $d$, cellules moyennes juxtacostales $\times$ 30o. e, cellules basilaires juxtacostales $\times 300 .-4$. Neckera madagassa Besch. - $a a^{3}$, feuille caulinaire $\times 24 b$, fragment du sommet de la feuille $\times 300, c$, cellules marginales moyennes $\times 300 . d$, cellules basilaires juxtacostales $\times 300$. $a^{\prime \prime}$, feuille caulinaire de $N$. fluminalis $\times 24 . f f^{\prime} f^{\prime \prime}$, folioles périchétiales de $N$. fluminalis $\times 24 . g$, capsule de $N$. fluminalis $\times 24$. - 5. Neckera pygmaa R. C. - $a a^{\prime} a^{\prime \prime}$, feuille caulinaire $\times 24$ (Diego Suarez). $a^{\prime \prime \prime}$, feuille caulinaire $\times 40 . b$, fragment du sommet de la feuille $\times 300 . c c^{\prime}$, cellules moyennes $\times 300 . d$, cellules des angles $\times 300 . e$, feuille caulinaire $\times$ 24 (Monts Ankaratra).

PI. 85. - I. Nechera Comora C. M. $-a a^{\prime}$, ferille caulinaire $\times 24 . b$, fragment sommet de la feuille $\times 300$. $c$, cellules marginales moyennes $\times 300$. $d$, cellules moyennes $\times 300$. $e e^{\prime} e^{\prime \prime}$, foliole périchétiale $\times 24 \cdot e^{\prime \prime}$, folioles périchétiales, archégones et paraphyses $\times 24 . f$, capsule $\times 24 . g$, fragment de péristome et exothecium $\times 1$ 70. $h$, spores $\times 300.2$. Neckera Borgeni Kiaer. $a a^{\prime}$, feuille caulinaire $\times 24 . b$, sommet de la feuille $\times$ i 70 . c, cellules marginales moyennes $\times 300, d$, foliole périgoniale $\times 40, e$, anthéridie $\times 40, f$ 
$g$, capsule $\times 24 . h$, fragment de péristome $\times 170, i$, spores $\times 300 .-3$. Neckera Pervillei Besch. - $a$, feuille caulinaire $\times 24 . b$, cellules moyennes $\times 300 . c c^{\prime} c^{\prime \prime}$, feuille raméale $\times 24$.

P1. 86. - 1. Homalia Valentini Besch. $-\approx a^{\prime}$, feuille caulinaire $\times 40 . b$, fragment du sommet de la feuille $\times 300$. $c$, cellules moyennes marginales $\times$ $300 . d$, cellules moyennes juxtacostales $\times 300 .-2$. Porotrichum Comorense C. M. $-a$, feuille caulinaire $\times 40 . b$, sommet de la feuille $\times 170$. c, cellules moyennes $\times 300 . d d^{\prime}$, feuille raméale $\times 40 . e$, sommet de la feuille $\times$ i 70 . $f$, cellules marginales vers le $1 / 4$ inférieur $\times 300 . g$, celluies des angles $\times 300$. - 3. Porotrichum mucronulatulum C. M. $-a$, feuille caulinaire $\times 40 . b$, cellules moyennes $\times 300 . c c^{\prime} c^{\prime \prime}$, feuille raméale $\times 40$. $d$, cellules moyennes $\times 300 .-4$. Porotrichum madagassum Kiaer. $-a a^{\prime}$, feuilles du stipe $\times 24$ (Borgen). $b$, cellules moyennes $\times 300$ (Borgen). $c$, feuille caulinaire $\times 24$ (Borgen). $d$, cellules moyennes $\times 300$ (Borgen). $f f^{\prime}$, feuille caulinaire $\times 24$ (Ambondromba). $g$, sommet de la feuille $\times 170$ (Ambondromba). $h$, cellules supérieures $\times 300 . i$, cel'ules marginales supérieures $\times 300 . j$, cellules moyennes juxtacostales $\times 300$. $k$, cellules des angles $\times 300 . l$, feuille caulinaire $\times$ 24 (Ankadivavala). $m$ m', feuille raméale $\times 24$ (Ankadivavala). $n$, cellules moyennes $\times 300$. (Ankadivavala).

Pl. 87.- I. Porotrichum subsecundum Kiaer. - $a$ a' $a$ ", feuille caulinaire $\times$ 24. $b$, cellules moyennes $\times 300 .-2$. Porotrichum Chenagoni C. M. $-a a^{\prime} a^{\prime \prime}$, feuille caulinaire $\times 40$. $b$, fragment du sommet de la feuille $\times 300$. $c$, cellules moyennes juxtacostales $\times 300 . d$, cellules basilaires juxtacostales $\times 300 . e$, cellules des angles $\times 300 . f f^{\prime} f^{\prime \prime}$, feuille raméale $\times 40 . g$, fragment du sommet de la feuille $\times 300, h$, cellules marginales suprabasilaires $\times 300 .-3$. Porotrichum tamariscinum $\mathrm{Hpe}$. $-a$, feuille du stipe $\times 40 . a^{\prime} a^{\prime \prime} a^{\prime \prime}$, feuille caulinaire $\times 40 . b$, sommet de la feuille $\times$ i $70 . c$, cellules moyennes marginales $\times 300 . d$, cellules moyennes juxtacostales $\times 300 . e$, cellules des angles $\times 300$. $f f^{\prime} f^{\prime \prime} f^{\prime \prime}$, feuille raméale $\times 40 . g$, sommet de la feuille $\times$ i 70 .

P1. 88. - 1. Porotrichum scaberulum R. C. - a a' $a^{\prime \prime}$, feuille caulinaire $\times 40 . b$, sommet de la feuille $\times 170 . c$, cellules moyennes marginales $\times 300$. $d$, cellules moyennes $\times 300$. $e$, cellules moyennes juxtacostales $\times 300 . f$, cellules des angles $\times 300 . g g^{\prime \prime} g^{\prime \prime \prime} g^{\prime \prime \prime \prime}$, feuille raméale $\times 40$. $h$, sommet de la feuille $\times 170 . i$, cellules moyennes $\times 300 . j$, périchèze $\times 40 . k k^{\prime}$, foliole périchétiale $\times 40 . l l^{\prime}$, capsule $\times 24 . m$, fragment de péristome et exothecium $\times 170, n$, spores $\times 300$.

P1. 89. - I. Fabronia Campenoni R. C. - $a$ a' $a "$, feuille caulinaire $\times$ 8o. $b$, sommet de la feuille $\times 170 . c$, cellules moyennes $\times 300$. $d$, cellules des angles $\times 300 . e$, foliole périgoniale et anthéridie $\times 170 . f$, capsule $\times 24 . g$, fragment de l'exothecium. $h$, spores $\times 300 .-2$. Fabronia fastigiata R. C. - $a a^{\prime}$, feuille caulinaire $\times 80$. $b$, sommet de la feuille $\times 170 . c$, cellules 
moyennes $\times 300$. $d$, cellules moyennes marginales $\times 300$. $e$, cellules des angles $\times 300 . f f^{\prime} f^{\prime \prime}$, capsule $\times 24 . g$, fragment du péristome et d'exothecium $\times 170 . h$, spores $\times 300,-3$. Fabronia crassiretis R. C. $-a a^{\prime}$, feuille caulinaire $\times 80 . b$, sommet de la feuille $\times$ i $70 . c$, cellules moyennes juxtacostales $\times 3$ oo. $d$, cellules moyennes marginales $\times 300$. $e$, cellules des angles $\times 300$.

Pl. 9o. - I. Fabronia Motelayi R. C. $-a$ ', feuille caulinaire $\times 80 . b$, sommet de la feuille $\times 300 . c$, cellules moyennes $\times 300$. $d$, cellules des angles $\times 300 . e$, foliole périchétiale $\times 80 . f$, capsule $\times 24 . g$, cellules de l'exothecium $\times 300 . h$, fragment du péristome $\times 1$ jo. $i$, spores $\times 300,-2$. Helicodontium fabroniopsis C. M. - $a$ ', feuille caulinaire $\times 80$. $b$, sommet de la feuille $\times$ 300 . $c$, cellules moyennes $\times 300$. $d$, cellules des angles $\times 300$. $e$, foliole périgoniale et anthéridie $\times 80 . f$, foliole périchétiale $\times 80 . g$, capsule $\times 24 . h$, cellules de l'exothecium $\times 300 . j$, fragment de péristome $\times 170 . k$, fragment d'une dent externe $\times 300$. l, fragment du péristome interne $\times 170 . m$, spores $\times 300$.

P1. 91. - I. Pseudoleskea subfilamentosa Kiaer. - $a a^{\prime} a^{\prime \prime}$, feuille caulinaire $\times 40 . b$, sommet de la feuille $\times$ r $70, c$, cellules marginales $\times 300 . d$, cellules moyennes juxtacostales $\times 300$. $e$, cellules basilaires juxtacostales $\times$ 30o. $f$, foliole périchétiale $<40 . g$, cellules juxtacostales $\times 300 .-2$. Thuidium subscissum C. M. - $a$, feuille caulinaire $\times 40 . a^{\prime} a^{\prime \prime}$, feuille caulinaire $\times 80 . b$, sommet de la feuille $\times 300 . c$, tissu vers le $1 / 4$ supérieur $\times 300 . d$, fragment de nervure $\times 300$. $e$, feuille raméale $\times 40 . e^{\prime} e^{\prime \prime}$, feuille raméale $\times 80$. $f$, feuille ramulinale $\times 170 . g$, sommet de la feuille $\times 300 . h$, cellules moyennes $\times 300 . i$, paraphylles $\times 300 . j j$ ' capsule $\times 24 . k$, foliole périchétiale $\times 80$. $l$, péristome et exothecium $\times$ 170. $m$, spores' $\times 300 .-3$. Thuidium Chenagoni C. M. $-a$, feuille caulinaire $\times 40 . a^{\prime} a^{\prime \prime}$, feuille caulinaire $\times 80, b b^{\prime}$, feuille ramulinale $\times$ izo. $c$, sommet de la feuille $\times 300$. $d$, tissu vers le $1 / 4$ supérieur $\times 300 . e$, paraphylles $\times 300$.

P1. 92. - I. Thuidium aculeoserratum R. C. $-a a^{\prime}$, feuille caulinaire $\times$ 40. $b b^{\prime}$, sommet de la feuille $\times 170 . c$, marge de la feuille vers le milieu $\times 300$. $d$, fragment de nervure $\times 170 . e e^{\prime}$, feuille raméale $\times 40 . f f^{\prime} f^{\prime \prime} f^{\prime \prime \prime}$, feuille ramulinale $\times 40 . g g^{\prime}$, feuille ramulinale $\times 170$. $h$, sommet de la feuille $\times 300$. $i$, cellules moyennes juxtacostales $\times 300 . j$, cellules marginales $\times 300 . k$, paraphylle $\times 300$. -2 . Rhegmatodon secundus Kiaer. - a a' $a^{\prime \prime}$, feuille caulinaire $\times 40 . b$, cellules marginales moyennes $\times 300 . c$, cellules juxtacostales moyennes. $d$, cellules basilaires $\times 300$.

P1. 93. - I. Macrohymenium acidodon Montg. - $a a^{\prime} a^{\prime \prime} a^{\prime \prime \prime}$ feuille caulinaire $\times 40 . b$, cellules marginales moyennes $\times 300 . c c^{\prime}$, cellules moyennes $\times 300 . d$, cellules des angles $\times 300 . e e^{\prime}$, foliole périchétiale $\times 40 . g$, cellules moyennes $\times 300 . h h^{\prime}$, capsule $\times 24 \cdot i$, fragment de péristome vu sur la face 
interne $\times \$ 70 . j$, membrane basilaire $\times 170 . k$, dents externes du péristome $\times 170.1$, spores $\times 300$.

Pl. 94. - 1. Leptohymenium fabronoïdes C. M. - $a a^{2} a^{\prime \prime}$, feuille caulinaire $\times 40 . b b$, feuille raméale $\times 40 \quad c$, cellules moyennes $\times 300$. $d$, cellules des angles $\times 300$. $e e^{\prime}$. foliole d'un périchèze $\times$ 40. $f$, cellules marginales moyennes $\times 300$. -2 Leptohymenium Borgeni (C. M.) Ren. $-a$ ', feuille caulinaire $\times 40 . b$, cellules moyennes $\times 300 . c$, cellales marginales moyennes $\times 300, d$, cellules des angles $\times 300 . e$, feuille raméale $\times 40 .-3$. Leptohymenium Ferrieżii Besch. $-a a^{\prime} a^{\prime \prime}$, feuille caulinaire $\times 40 . \quad b b^{\prime}$, feuille raméale $\times 40 . c c^{\prime} c^{\prime \prime}$, cellules marginales moyennes $\times 300$. $e$, archégone $\times 80$. - 4. Leptohymenium dilatatum R. C. - a $a^{\prime} a^{\prime \prime}$, feuille caulinaire $\times 40 . b b^{\prime}$, feuille raméale $\times 40$. c, cellules marginales moyennes $\times 300 . d d^{\prime} d^{\prime \prime}$, cellules moyennes $\times 300 . e$, cellules des angles $\times 300$.

Pl. 95. - I. Leptohymenium Ferriezii Besch., var, abbreviatum R. C. $a a^{\prime}$, feuille caulinaire $\times 40$. $c$, cellules marginales vers le $\mathrm{s} / 4$ supérieur $\times 300$. $c$, cellules moyennes $\times 300 . d$, cellules des angles $\times 300$. $e e^{\prime} e^{\prime \prime}$, feuille raméale $\times 4$ o. $f$, cellules marginales moyennes $\times 300 . g$, cellules moyennes $\times 300 . h h^{\prime} h^{\prime \prime}$, foliole périchétiale $\times 40$. $i$, cellules marginales d'une foliole $\times$ $300 . j j$, capsule $\times 24 . k$, fragment de péristome $\times$ i 70. $l$, spores $\times 300 .-$ 2. Entoton Corbieri R. C. $-a a^{\prime} a^{\prime \prime} a^{\prime \prime \prime}$, feuille caulinaire $\times 40$. $b$, sommet de la feuille $\times 300 . b^{\prime}$, sommet de la feuille $\times$ r $70 . c$, cellules moyennes $\times 300$. $d$, cel'ules des ángles $\times 300$. c cellules marginales vers les $2 / 3$ supérieurs $X$ 30o. $f f^{\prime}$, foliole périchétiale $\times 40 . g$, capsule $\times 24 . h$, fragment de péristome $\times$ r $70 . i$, spores $\times 300$.

Pl. 96. - I. Entodon Felicis. R.C. - $a a^{\prime}$, feuille caulinaire $\times 40 . b$, feuille raméale $\times 40 . c c^{\prime}$, foliole périgoniale $\times 40 . d d^{\prime}$, foliole périchétiale $\times 40 . e$, capsule $\times 24 . f$, fragment de péristome $\times 170 . g$, fragment d'anneau $\times 170$. $h$, spores $\times 300$. $i i^{\prime} i^{\prime \prime}$, feuille caulinaire $\times 40$ (var. julaceum). $j j$ ', sommet de la feuille $\times 300 . k$, cellules moyennes $\times 300$. $l$, cellules marginales moyennes $\times 300 . m$, cellules des angles $\times 300 . n n^{\prime}$, feuille raméale $\times 40.0$, sommet de la feuille $\times$ 170. - 2. Entodon Kutenbergii C. M. - a, feuille caulinaire $\times 4$ o. $a^{\prime} a^{\prime \prime}$, feuille raméale $\times 40 . b b^{\prime}$, sommet de la feuille $\times 300$. $c$, cellules moyennes $\times 300, d$, cellules des angles $\times 300$.

P1. 97. - I. Entodon Rutenbergii C. M. $-a a^{\prime}$, foliole périchétiale $\times 40$. $b b^{\prime}$, capsule $\times 24, c$, fragment de péristome $\times$ i $70, c c^{\prime}$, dent externe $\times$ i 70 . $d$, spores $\times 300 .-2$. Lindigia Hildebrandtii C. M. $-a a^{\prime}$, feuille caulinaire $\times$ 24. $b$, sommet de la feuille $\times$ i 70 . $c$, cellules moyennes marginales $\times 300 . d$, cellules des angles $\times 300 . e$, foliole périchétiale $\times 24 . f f^{\prime}$, capsule $\times 24 . g$, fragment de péristome $\times 170 . h$, spores $\times 300 .-3$. Sematophrllum megas porum Duby. - $a a^{\prime}$, feuille caulinaire $\times 24 . b$, cellules moyennes $\times 300 . c$, cellules des angles $\times 170$. $d d^{\prime}$, foliole périchétiale $\times 40$. e, sommet de la 
foliole $\times 170 . f$, capsule $\times 24 . g$, pédicelle $\times 170 . h$, fragment de péristome $\times$ i 70 , $i$, spores $\times 300$.

Pl. 98. - 1. Sematophyllum megasporum var. densum R. C. - a $a^{\prime} a^{\prime \prime}$, feuille caulinaire $\times 24, b$, cellules des angles $\times 1$ 70, -2 . Sematophyllum stellatum R. C. - $a a^{\prime} a^{\prime \prime} a^{\prime \prime \prime}$, feuille caulinaire $\times 24$. $b$, cellules moyennes $\times$ 300. $c$, cellules des angles $\times$ i 70. -3 . Sematophrllum rufoviride Besch. $a a^{\prime}$, feuille caulinaire $\times 24 \cdot a^{\prime \prime} a^{\prime \prime}$, feuille caulinaire $\times 40 . b$, sommet de la feuille $\times$ i $70 . c$, cellules moyennes $\times 300 . d$, cellules des angles $\times 300 .-4$. Sematophyllum subscabrellum R. C. $-a a^{\prime} a^{\prime \prime}$, feuille caulinaire $\times 24 \cdot a^{\prime \prime}$, feuille caulinaire $\times 40 . b$, sommet de la feuille $\times$ 1 $70 . c$, marge de la feuille montrant les papilles $\times 3$ oo. $d$, cellules moyennes $\times 3$ oo. $e$, cellules des angles $\times 300$. - 5. Sematophyllum lavifolium R. C. $-a a^{\prime}$, feuille caulinaire $\times 24$. $a^{\prime \prime}$, feuille caulinaire $\times 4$ o. $b b^{\prime}$, sommet de la feuille $\times$ I70. $c$, cellules moyennes $\times 300 . d$, cellules des angles $\times 300$, $e$, capsule $\times 24 .-6$. Sematophyllum Rutenbergii C. M. $-a a^{\prime}$, feuille caulinaire $\times 40 . b b^{\prime}$, feuille raméale $\times 40$. c , sommet de la feuille $\times$ i 70. $d$, cellules moyennes $\times 300$. $e$, cellules des angles $\times 300$.

Pl. 99. - 1. Sematophyllum protensum R. C. - $a a^{\prime} a^{\prime \prime} a^{\prime \prime \prime}$, feuille caulinaire $\times 40, b b^{\prime}$, sommet de la feuille $\times$ r 7o. c, cellules moyennes $\times 300 . d$, cellules"des angles $\times 300$. $e e^{\prime} e^{\prime \prime} e^{\prime \prime}$, feuille raméale $\times 40 . f$, sommet de la feuille $\times$ I $70 . \mathrm{g}$, cellules des angles $\times 300 .-2$. Sematophyllum Bessoni R. C. $-a a^{\prime} a^{\prime \prime}$. feuille caulinaire $\times 40 . b b^{\prime}$, sommet de la feuille $\times 170 . c$, cellules moyennes $\times 300$. $d d^{\prime}$, cellules des angles $\times 300 . e e^{\prime}$, feuille raméale $\times$ 40. $f$, cellules moyennes $\times 300 . g$, sommet de la feuille $\times 170 .-3$. Sematophyllum flexile R. C. $-a a^{\prime} a^{\prime \prime} a^{\prime \prime}$, feuille caulinaire $\times 40 . b b^{\prime}$, sommet de la feuille $\times$ i 70. $c$, cellules moyennes $\times 300, d$, cellules des angles $\times 300$. $e e^{\prime}$, feuille raméale $\times 40 . f$, cellules moyennes $\times 300 . g g^{\prime}$, cellules des angles $\times 300$.

P1. I00. - 1. Raphidostegium Duisaboanum Besch. - $a a^{\prime}$, feuille caulinaire $\times 40 . b$, cellules supérieures $\times 300 . c$, cellules marginales moyennes $\times 300 . d$, cellules moyennes $\times 300 . e$, cellules des angles $\times 300 . f$, foliole périchétiale $\times 40 . g g^{\prime}$, capsule $\times 24 . h$, fragment de péristome $\times 170$. $i$, spores $\times 300 . j$, feuille caulinaire $\times 40$ (var. Nossianum). $k$, cellules moyennes $\times 300 . l$, cellules des angles $\times 300 . m m^{\prime}$, feuille caulinaire (var. argillicola). $n$, cellules moyennes $\times 300.0$, cellules des angles $\times 300 . p p^{\prime}$, capsule $\times 24 . q$, fragment de péristome $\times 1$ 70. $r$, spores $\times 300 .-2$. Raphidostegium ovalifolium Besch. - $a a^{\prime} a^{\prime \prime} a^{\prime \prime \prime}$, feuille caulinaire $\times 40$. $b$, sommet de la feuille $\times$ I 70. $c$, cellules moyennes $\times 300 . d$, cellules marginales moyennes $\times 300 . e e^{\prime}$, cellules des angles $\times 300$.

Pl. го1. - 1. Raphidostegium rubricaule Besch. - $a a^{\prime} a^{\prime \prime}$, feuille caulinaire $\times 40 . b b^{\prime}$, sommet de la feuille $\times$ i 70 . $b^{\prime \prime}$, sommet de la feuille $\times 300$. 
$c$, cellules moyennes $\times 300$. $d$, cellules des angles $\times 300 .-2$. Raphidostegium Pervilleanum Schimp. - $a a^{\prime}$, feuille caulinaire $\times 40 . b b^{\prime}$, sommet de la feuille $\times$ i $70 . c, d$, cellules moyennes $\times 300 . e$, cellules des angles $\times 300$. $f$, foliole périchétiale $\times 40, f^{\prime}$, sommet d'une foliole $\times 170, g g$ ', capsule $\times$ 24. $h$, fragment de péristome $\times$ i 70 . $i$, spores $\times 300 .-3$. Raphidostegium Cambouei R. C. $-a a^{\prime}$, feuille caulinaire $\times 40 . a^{\prime \prime} a^{\prime \prime}$, feuille caulinaire $\times 80$. $b$, sommet de la feuille $\times$ i 70. $b$, sommet de la feuille $\times 300$. $c$, cellules moyennes $\times 300$. $d d^{\prime}$, cellules des angles $\times 300$. e, capsule $\times 24 .-4$. Sematophyllum laevifolium R. C. $-a a^{\prime}$, feuille caulinaire $\times 24 . b$, sommet de la feuille $\times$ 170. $c c^{\prime}$, foliole périchétiale $\times 40 . d d^{\prime}$, capsule $\times 24 . e$, fragment de péristome $\times 170$ (capsule vieille). $f$, fragment de l'exothecium $\times 300 . g$, spores $\times 300 . h$, fragment de péristome $\times 170$ (capsule verte). $i$, spores $\times 300$.

PI. 102. - I. Raphidostegium dubium C. R. - $a$ a' $a$ " $a$ "', feuille caulinaire $\times 40 . b$, sommet de ia feuille $\times 170 . c$, cellules moyennes $\times 300, d$, base de la feuille $\times 300$. e e $e^{\prime \prime}$, foliole périchétiale $\times 40$. $f f^{\prime}$, capsule $\times 24$. $g$, fragment de péristome $\times 170 . h$, spores $\times 300 .-2$. Raphidostegium Peryilleanum Sch. (Ech. Pervillé). - $a a^{\prime} a^{\prime \prime}$, feuille caulinaire $\times 40$. $b$, sommet de la feuille $\times 170 . c c^{\prime}$, cellules moyennes $\times 300 . d$, cellules des angles $\times 300$. $e e^{\prime}$, capsule $\times 24 . f$, fragment du péristome $\times$ i $70 . f$, portion d'une dent externe $\times 300 . h$, spores $\times 300$.

P1. I03. - I. Trichosteleum microdontium Besch. - a a' $a^{\prime \prime} a^{\prime \prime}$, feuille caulinaire $\times 40 . b$, sommet de la feuille $\times \mathrm{r}_{70}$. cellules marginales supérieures $\times 300 . d$, cellules moyennes $\times 300 . e$, cellules marginales inférieures $\times 300 . f f^{\prime}$, cellules des angles $\times 300 . g$, papilles en saillie sur la face dorsale de la feuille $\times 300 . h h^{\prime} h^{\prime \prime} h^{\prime \prime \prime}$, foliole périchétiale $\times 4$ o. $i$, cellules marginales supérieures d'une foliole $\times 300 . j j$ ', capsule $\times 24$. $k$, fragment du péristome $\times 170 . l$, spores $\times 300 . m$, sommet d'une foliole périgoniale $\times 170 .-2$. Trichosteleum microdontium var. megapteron R. C. - a a' $a^{\prime \prime}$, feuille caulinaire $\times 40 . b b^{\prime}$, saillie dorsale des papilles $\times 300 . c$, cellules des angles $\times 300$. - 3. Trichosteleum trachypyxis C. M. (Rutenberg). - $a a^{\prime} a^{\prime \prime}$, feuille caulinaire $\times 40 . b b^{\prime}$, sommet de la feuille $\times$ i 70 . $c$, cellules marginales supérieures $\times 300 . d$, cellules marginales moyennes $\times 300$. $e$, cellules moyennes $\times 300 . f$, cellules des angles $\times 300 . m$, saillie aculeiforme des papilles dorsales $\times 300 . g g^{\prime}$, feuille caulinaire $\times 40$ (Analamazoatra). $h$, cellules marginales moyennes $\times 300$. $i$, cellules marginales inférieures $\times 300 . j$, sommet de la feuille $\times$ i $70 . k$, cellules des angles $\times 300 . l$, saillie des papilles dorsales $\times$ 300 .

P1. I04. - I. Trichosteleum Perroti R. C. - $a a^{\prime} a^{\prime \prime} a^{\prime \prime \prime}$, feuille caulinaire $\times 40 . b$, sommet de la feuille $\times 1$ 70. $c c^{\prime}$, cellules moyennes $\times 300 . d$, cellules marginales moyennes $\times 300 . e e^{\prime}$, cellules des angles $\times 300 . f f^{\prime} f^{\prime \prime}$, foliole périchétiale $\times 40 . g$, cellules marginales supérieures $\times 300$. $h h^{\prime}$, cap- 
sule $\times 24$. $i$,fragment du péristome $\times$ i 7o. $j$, spores $\times 300 .-2$. Trichosteleum Perrotivar. eurydictyon R.C. $-a a^{\prime} a^{\prime \prime}$, feuille caulinaire $\times 40 . b$, sommet de la feuille $\times$ r $70 . c$, cellules marginales et pli $\times 300 . d$, saillie dorsale des papilles $\times 300$. e $e^{\prime}$, cellules moyennes $\times 300 . f f^{\prime}$, cellules des angles $\times 300$. 3. Trichosteleum adharens Besch. $-a a^{\prime} a^{\prime \prime}$, feuille caulinaire $\times 40, b b^{\prime}$, sommet de la feuille $\times 170$. $c$, cellules moyennes $\times 300$. $d$, cellules marginales moyennes $\times 300$. $e$, cellules des angles $\times 300$.

P1. 105. - I. Taxithelium serratum R. C. $-a$ ', feuille caulinaire $\times 40$. (Mahambo). $b$, sommet de la feuille $\times$ i 7o. $c$, cellules marginales moyennes $\times 300$. $d$, cellules moyennes $\times 300$, e, cellules des angles $\times 300 . f f^{\prime}$, saillie dorsale des papilles $\times 300 . g g^{\prime} g^{\prime \prime} g^{\prime \prime \prime}$, fenille caulinaire $\times$ 40 (sine loco, Friren), $h$, sommet de la feuille $\times 170$. $i$ ', cellules marginales moyennes $\times$ $300 . j j$, cellules moyennes $\times 300$. $k$, cellules des angles $\times 300 .-2$. Taxithelium (?) Loucoubense Besch. $-a a^{\prime} a^{\prime \prime}$, feuille caulinaire $\times 40$. $b$, sommet de la feuille $\times$ I $70 . c c^{\prime}$, cellules moyennes $\times 300 . d$, cellules des angles $\times 300 . e$, saillie dorsale des papilles $\times 300 . f$, fragment de péristome $\times$ i 70 . $g$, portion d'une dent externe $\times 300$. $h$, spores $\times 300$. $i$, capsule $\times 24 .-3$. Taxithelium decolor Besch. - $a a^{\prime} a^{\prime \prime} a^{\prime \prime}$, feuille caulinaire $\times 40, b b^{\prime}$, sommet de la feuille $\times 1$ 70. $c$, cellules moyennes $\times 300$. $d$, cellules marginales moyennes $\times 300$. $e$, cellules des angles $\times 300$.

Pl. 106.- - . Trichosteleum leptorrhynchum. $-a a^{\prime}$, feuille caulinaire $\times 40$ (La Réunion). $b$, sommet de la feuille $\times$ I $70 . c$, saillie dorsale des papilles $\times$ 30o. $d$, capsule $\times 24 . e$, fragment de péristome $\times 170 . f$, portion d'une dent externe $\times 300 . g$, spores $\times 300$. madagassum R. C. $-a a^{\prime} a^{\prime \prime} a^{\prime \prime}$, feuille caulinaire $\times 40$ (Analamazoatra). $b$, cellules moyennes $\times 300$. $c$, cellules des angles $\times 300$. $d$, saillie dorsale des papilles $\times 300 . e$, foliole périchétiale $\times 40 . f$, sommet de la foliole $\times 170 . g$, cellules moyennes marginales de la foliole $\times 300$. $h h$, capsule $\times 24$. $i$, fragment de péristome $\times$ r $70 . j$, portion de l'exothecium $\times 300$. $k$, spores $\times 300$. l l' $l^{\prime \prime}$, feuille caulinaire $\times 40$ (Pervillé). $n$, saillie dorsale des papilles $\times 300$. $m$, cellules moyennes $\times 300$. o, fragment de péristome $\times 170$.

P1. 107. - I. Trichosteleum borbonicum Bel. $-a a^{\prime}$, feuille caulinaire $\times 40$ (La Réunion). $a$ " $a$ "', feuille caulinaire $\times 40$ (Analamazoatra). $b$, sommet de la feuille $\times$ r 70. $c c^{\prime}$, capsule $\times 24 . d^{\prime} d^{\prime \prime}$, feuille caulinaire $\times 40$ (var. brachycarpum). $e$, cellules moyennes $\times 300 . f$, cellules suprabasilaires $\times 300 . g$, cellules des angles $\times 300 . h h^{\prime}$, foliole périchétiale $\times 40$. $i$, sommet d'une foliole $\times$ i 70. $j$, marges de la foliole $\times 170 . k k^{\prime} k^{\prime \prime}$, capsule $\times 24 . l$, fragment de péristome $\times 170 . m$, spores $\times 300$. -2 . Taxithelium planulum Besch. - $a a^{2}$, feuille caulinaire $\times 40 . b$, sommet de la feuille $\times 170, c$, cellules moyennes $\times 300 . d$, cellules marginales moyennes $\times 300$. e $e$, cellules des angles $\times 300 . f$, capsule $\times 24 .-3$. Taxithelium laetum R. C. $-a a^{\prime} a^{\prime \prime} a^{\prime \prime}$, 
feuille caulinaire $\times 40$. $b$, sommet de la feuille $\times$ r $70 . b^{\prime}$, sommet de la feuille $\times 300 . c$, cellules moyennes $\times 300 . d$, foliole périchétiale $\times 40 . e e^{\prime}$, capsule $\times 24$. -4 . Taxithelium glaucophrllum Besch. $-a a^{\prime}$, feuille caulinaire $\times 40 . b$, sommet de la feuille $\times$ i $70 . b^{\prime}$, sommet de la feuille $\times 300 . c c^{\prime}$, cellules moyennes $\times 300$. $d$, cellules des angles $\times 300$.

PI 108.- I. Taxithelium nossianum Besch.- $a a^{\prime} a^{\prime \prime} a^{\prime \prime}$, feuille caulinaire $\times$ 40. $b$, sommet de la feuille $\times 300 . c$, cellules marginales moyennes $\times 300$. $d$, cellules moyennes $\times 300 . e$, cellules des angles $\times 300 . f$, capsule $\times 24$. 2. Taxithelium argyrophyllum R. C. $-a$, feuille caulinaire $\times 40$. $b b$, sommet de la feuille $\times 300 . c$, cellules moyennes $\times 300 . d$, cellules des angles $\times 300 . e e^{\prime}$, foliole périchétiale $\times 40 . f$, portion de l'acumen $\times 300 . g$, base de l'acumen $\times 300 . h h^{\prime} h^{\prime}$, capsule $\times 24$. $i$, fragment du péristome $\times 170 . j$, spores $\times 300 .-3$. Taxithelium serratum $\mathrm{R}$. C. $-a$, capsule $\times 24 . b$, fragment de péristome $\times 170$. $c$, portion d'une dent externe $\times 300$. $d$, spores $\times$ 300. - 4. Isopterygium gracile R. C. $-a a^{\prime}$, feuille caulinaire $\times 40$. $a^{\prime \prime} a^{\prime \prime \prime}$, feuille raméale $\times 40 . b$, sommet de la feuille $\times 170 . b^{\prime}$, sommet de la feuille $\times$ $300 . c c^{\prime}$, cellules moyennes $\times 300 . d$, cellules des angles $\times 300$.

$\mathrm{Pl}$. 109. - I. Isopterygium argyroleucum $\mathrm{C}$. M. - $a$, feuille caulinaire $\times 40$ (type de Maurice). $b$, sommet de la feuille $\times$ I $70 . b^{\prime}$, sommet de la feuille $\times 300 . c$, cellules moyennes $\times 300 . d$, cellules des angles $\times 300 . e$, capsule $\times$ 24. $f$, dent externe du péristome $\times$ i $70 . g$, spores $\times 300 . h$, feuille caulinaire $\times 40$ (Madagascar, Kiaer), $i$, sommet de la feuille $\times 300, j$, cellules moyennes $\times 300 . k$, capsule $\times 24 . l$, fragment de péristome externe $\times$ r $70 . l$, fragment de péristome interne $\times$ I $70 . m$, spores $\times 300 .-2$. Isopterygium leptoblastum C.M. $-a$, feuille caulinaire $\times 40$ (type d'Anjouan). $b$, sommet de la feuille $\times$ r $70, b^{\prime}$, sommet de la feuille $\times 300$. $c$, cellules marginales moyennes $\times 300$. $c^{\prime}$, cellules moyennes $\times 300$. $d d^{\prime}$, cellules des angles $\times 300$. $e e^{\prime} e^{\prime \prime}$, foliole périchétiale $\times 40 . f$, capsule $\times 24 . g$, fragment de péristome $\times{ }^{1} 70$. $h$, spores $\times 300 . i$, feuille caulinaire $\times 40$ (Mayotte). $j j$, sommet de la feuille $\times 300$. $k$, cellules moyennes $\times 300$.

Pl. I 10. - I. Isopterygium subleptoblastum Besch. - $a$, feuille caulinaire $\times$ 40. $b$, sommet de la feuille $\times \times$ r $0 . b^{\prime}$, sommet de la feuille $\times 300 . c$, cellules moyennes $\times 300 . d$, cellules des angles $\times 300 . e e^{\prime}$, foliole périchétiale $\times 40$. $f$, fragment de péristome externe $\times$ i $70 . g$, capsule $\times 24 . h$, spores $\times 300$. 2. - Isopterygium Boivini Besch. $-a$, feuille caulinaire $\times 40$ (Sainte-Marie). $b$, sommet de la feuille $\times 300$. $c$, cellules marginales moyennes $\times 300$. $d$, feuille caulinaire $\times 40$ (Nossi Comba). $e$, feuille caulinaire $\times 40$ (Diego Suarez). $f$, cellules moyennes $\times 300 . g$, capsule $\times 24 .-3$. Isopterygium Boivini var. terrestre $\mathrm{R}$. C. $-a$, feuille caulinaire $\times 40 . a^{\prime}$, feuille raméale $\times 40 . b$, sommet de la feuille $\times$ ז $70, b^{\prime}$, sommet de la feuille $\times 300$. $c$, cellules marginales moyennes $\times 300, d d^{\prime} d^{\prime \prime}$, cellules moyennes $\times 300$. $e$, cellules des angles $\times$ 


\section{DES MASCAREIGNES ET DES COMORES}

30o. $f f^{\prime} f^{\prime \prime}$, foliole périchétiale $\times 40 . g$, cellules moyennes d'une foliole $\times$ 30o. $h$, capsule $\times 24 . i$, fragment de péristome $\times$ i $70 . j$, spores $\times 300$.

Pl. I г. - I. Isopterygium (?) appressum R. C. - $a$, feuille caulinaire $\times 40$. $b$, feuille raméale $\times 40$. $c$, sommet de la feuille $\times 300$. $d$, cellules moyennes $\times 300, e$, cellules des angles $\times 300 .-2$. Isopterygium intortum P. Beauv. $-a$, feuille caulinaire $\times 40$ (Maurice). $b b^{\prime}$, sommet de la feuille $\times$ 170. $c c^{\prime} c^{\prime \prime}$, cellules moyennes $\times 300 . d d^{\prime}$, cellules des angles $\times 300 . a^{\prime}$, feuille caulinaire $\times 40$ (La Réunion). $e$, foliole périchétiale $\times 40 . f$, portion de l'acumen d'une foliole $\times 170 . g g^{\prime}$, capsule $\times 24 . h$, fragment de péristome $\times 170$. $i$, spores $\times 300 . j$, feuille caulinaire $\times 40$ (var Chenagoni $)$. $k$, cellules moyennes. -3 . Isopterygium minutum R. C. $-a$, feuille caulinaire $\times 40 . b b$, sommet de la feuille $\times 300$. $c$, cellules moyennes $\times 300 . d$, foliole périchétiale $\times 40 . e$, sommet d'une foliole $\times 300 . f f$ ', capsule $\times 24 . g$, fragment de péristome $\times 170 . h$, spores $\times 300$.

Pl. I12. - I. Isopterygium Ambreanum R. C. $\rightarrow a$, feuille caulinaire $\times$ 40. $a^{\prime}$, feuille raméale $\times 40, b$, sommet de la feuille $\times 170 . b^{\prime}$, sommet de la feuille $\times 300, c$, cellules moyennes $\times 300, d$, cellules des angles $\times 300 .-2$. I. Boivini var. costatum R. C. $-a$, feuille caulinaire $\times 40, b$, sommet de la feuille $\times$ i $70, c$, cellules moyennes $\times 300 .-3$. Acrocladium Auberti. $-a$, feuille caulinaire $\times 40$ (La Réunion). $a^{\prime}$, feuille caulinaire $\times 40$ (Madagascar). $b$, sommet de la feuille $\times$ r $70 . c c^{\prime}$, cellules moyennes $\times 300 . d$, cellules des angles $\times 300 .-4$. Plagiothecium austrodenticulatum R. C. - a $a^{\prime} a^{\prime \prime} a^{\prime \prime \prime}$, feuille caulinaire $\times 40 . b b^{\prime}$, sommet de la feuille $\times 170 . c c^{\prime}$, cellules moyennes $\times 300$. $d$, cellules des angles $\times 300$.

Pl. I13. - 1. Ectropothecium regulare C. M. - a, feuille caulinaire $\times$ 40. $b b^{\prime}$, sommet de la feuille $\times$ i $70 . c c^{\prime}$, cellules moyennes $\times 300 . d$, foliole périchétiale $\times 40 . e$, base de l'acumen de la foliole $\times 300 . f$, cellules moyennes de la foliole $\times 300 . / t$, capsule $\times 24$, $i$, fragment de péristome $\times 170 . j$, spores $\times 300 .-2$. Ectropothecium nano-cristacastrensis C. M. $-a$, feuille caulinaire $\times 40 . b$, sommet de la feuille $\times 170 . c$, cellules des angles $\times 300 . d$, foliole périchétiale $\times 40, e$, base de l'acumen de la foliole $\times 300 .-3$. Ectropothecium Serchellarum Besch. $-a$, feuille caulinaire $\times 40 . b b^{\prime}$, sommet de la feuille $\times$ i $70 . c c^{\prime}$, cellules moyennes $\times 300, d$, tissu basilaire $\times 300$.

Pl. 114. - I. Ectropothecium Chenagoni R. C. - a, feuille caulinaire $\times$ 40. $b$, sommet de la feuille $\times$ i 70 . $b$ ', sommet de la feuille $\times 300$. $c$, cellules moyennes $\times 300, d$, cellules des angles $\times 300$. $e$, feuille caulinaire latérale de la var. hamatulum $\times 40 . e^{\prime}$, feuille caulinaire médiane de la var. hamatulum $\times$ 40. - 2. Ectropothecium Pailloti R. C. $-a$, feuille caulinaire $\times 40$. $a$, feuille raméale $\times 40, b$, sommet de la feuille $\times$ i $70 . c$, cellules moyennes $\times$ 3oo. - 3. Ectropothecium Perroti R. C. - a, feuille caulinaire latérale $\times$ 
40. $a^{\prime}$, feuille caulinaire médiane $\times 40 . b b^{\prime} b^{\prime \prime}$, sommet de la feuille $\times$ I $70 . c$, cellules moyennes $\times 300 . d d^{\prime}$, cellules des angles $\times 300 .-4$. Ectropothecium intertextum R. C. $-a$, feuille caulinaire latérale $\times 40 . a^{\prime}$, feuille caulinaire médiane $\times 40 . b b^{\prime}$ sommet de la feuille $\times$ i $70 . c c^{\prime}$, cellules moyennes $\times 300, d d^{\prime}$, foliole périchétiale $\times 40$.

Pl. I 5. - I. Ectropothecium arcuatum R. C. $-a$, feuille caulinaire latérale $\times 40$. $a^{\prime}$, feuille caulinaire médiane $\times 40$. $b$, sommet de la feuille $\times$ I 70. $c$, cellules moyennes $\times 300 . d$, cellules des angles $\times 300 . e$, foliole périchétiale $\times 40,-2$. Ectropothecium (?) podorrhizon R. C. $-a$, feuille caulinaire $\times 40, b$, sommet de la feuille $\times$ i $70 . b^{\prime}$, sommet de la feuille $\times 300 . c c^{\prime}$, cellules moyennes $\times 300 . d$, cellules des angles $\times 300 . e e^{\prime}$, foliole périchétiale $\times$ 40. $f$, sommet d'une foliole $\times$ I $70 . g$, capsule $\times 24 . h$, fragment de péristome $\times$ i $70 . i$, spores $\times 300 . j$, foliole périgoniale $\times 40 . k$, anthéridie $\times 40$.

P1. I 6 . - I. Ectropothecium sphaerocarpum C. M. - $a$, feuille caulinaire $\times 40 . b$, cellules moyennes $\times 300 . c$, foliole périchétiale $\times 40 . d$, capsule $\times 24$. $e$, fragment du péristome $\times$ I $70 . f$, spores $\times 300 .-2$. Ectropothecium crassirameum R. C. $-a$, feuille caulinaire $\times 40$ (Diego Suarez). $b$, cellules moyennes $\times 300$. $a^{\prime}$, feuille raméale $\times 40 . b^{\prime}$, cellules moyennes $\times 300 . a^{\prime \prime}$, feuille caulinaire $\times 40$ (Nossi Bé). $b^{\prime \prime}$, cellules moyennes $\times 300$. a", feuille raméale $\times 40$. b"', cellules moyennes $\times 300$.

P1. I I 7. - I. Ectropothecium galerulatum Duby. - a, feuille caulinaire $\times$ 40. $b$, cellules moyennes $\times 300, c$, capsule $\times 24 . d$, fragment du péristome $\times$ i $70 . e$, spores $\times 300 .-2$. Ectropothecium Ayresii $\mathrm{Sch} .-a$, feuille caulinaire $\times 40$ (type de Daruty). $b$, feuille caulinaire $\times 40$ (Robillard). $b$, feuille raméale $\times 40 . c c^{\prime}$, cellules moyennes $\times 300$. $d$, foliole périchétiale $\times 40 . c$, capsule $\times 24 . f$, fragment de péristome $\times 170 . g$, spores $\times 300$.

P1. I 8. - I. Ectropothecium alboviride Ren. - $a a^{\prime}$, feuille caulinaire $\times 40 . b$, cellules moyennes $\times 300 . c$, foliole périchétiale $\times 40 . d$, capsule $\times$ 24. $e$, fragment de péristome $\times$ 1 70. $f$, fragment de l'anneau triple $\times 300 . g$, spores $\times 300 .-2$. Ectropothecium subspharicum C. M. $-a$, feuille caulinaire $\times 40 . b$, base de la subule $\times 300, c c^{\prime}$, cellules moyennes $\times 300 . d$, cellules des angles $\times 300 . e e^{\prime}$, foliole périchétiale $\times 40 . f$, capsule $\times 24 . g$, fragment de péristome $\times 170 . h$, spores $\times 300$.

Pl. I I9. - I. Ectropothecium Bescherellei Ren. $-a$, feuille latérale $\times 40$. $a^{\prime}$, feuille médiane $\times 40 . b$. sommet de la feuille $\times 300 . c c^{\prime}$, cellules moyennes $\times 300, d d^{\prime}$. foliole périchétiale $\times 40, e e^{\prime}$, capsule $\times 24, f$, fragment de péristome $\times 170 . g$, spores $\times 300$.

Pl. 120. - I. Ectropothecium Rodriguezii R. C. - $a$, feuille caulinaire $\times 40$. $b$, sommet de la feuille $\times 300$. $c$, cellules moyennes $\times 300 . d d^{\prime} d^{\prime \prime}$, feuille caulinaire (var. pinnatum) $\times 40$. $e$, cellules moyennes $(1 d e m) \times$.300 . 
$f f^{\prime}$, foliole périchétiale $(I d e m) \times .40 . g g^{\prime}$, capsule $\times 24 . h$, fragment de péristome $\times 170 . i$, spores $\times 300 . j$, fragment d'anneau $\times 300$.

Pl. I21. - 1. Ectropothecium Comba R. C. $-a$, feuille caulinaire $\times 40$. $b$, sommet de la feuille $\times 300 . c$, cellules moyennes $\times 300 . d^{\prime} d^{\prime \prime}$, foliole périchétiale $\times 40 . e$, capsule $\times 24 . f$, fragment de péristome $\times 170 . g$, spores $\times 300 .-2$. Ectropothecium alboviride var. rufulum. - $a a^{\prime} a^{\prime \prime}$, feuille caulinaire $\times 4^{0} . b^{\prime} b^{\prime \prime}$, cellules moyennes $\times 300$.

Pl. 122. - 1. Stereophyllum Combaniense Besch. - $a$, feuille caulinaire $\times$ 40. $b$, sommet de la feuille $\times 300$. $c$, cellules moyennes $\times 300$. $d$, cellules marginales au-dessus de la base $\times 300$. $e$, cellules des angles $\times 300 . f$, foliole périchétiale $\times 24$. $i$, capsule $\times 24 . g$, fragment de péristome $\times{ }^{-1} 70 . h h^{\prime}$, spores $\times 300 .-2$. Hypnum Caussequei R. C. $-a$, feuille caulinaire $\times 40$. $b b^{\prime} b^{\prime \prime}$, sommet de la feuille $\times 170 . c c^{\prime} c^{\prime \prime}$, cellules moyennes $\times 300 . d$, cellules des angles $\times 300$.

P1. 123. - I. Hypnum aduncoüdes C. M. $-a$, feuille caulinaire $\times 40 . b$, sommet de la feuille $\times$ i $70 . c$, cellules marginales supérieures $\times 300 . d$, cellules moyennes $\times 300$. $e$, cellules des angles $\times$ i $70 . f$, feuille raméale $\times 40$. $g$, cellules moyennes $\times 300 . h h^{\prime}$, feuille caulinaire $\times 40$ (var. Kiaerii). $i$, sommet de la feuille $\times 170 . j$, cellules des angles $\times 1$ 70. $k$, feuille raméale $\times$ 40. -2 . Hypnum luteonitens $\mathrm{R}$. C. $-a$, feuille caulinaire $\times 40 . b$, sommet de la feuille $\times$ 170. $c c^{\prime}$, cellules moyennes $\times 300 . d$, cellules des angles $\times$ 30o. $e e^{\prime}$, foliole périchétiale $\times 40$.

Pl. I 24. - I. Rhacopilum proelongum Sch. $-a$, feuille caulinaire $\times 24$ (type de Richard). $b$, sommet de la feuille $\times 80$. $c$, cellules supérieures $\times 300$. $d$, cellules basilaires juxtacostales $\times 300$. $e e^{\prime}$, stipule $\times 24 . f$, sommet de la stipule $\times$ r 70. -2 . Rhacopilum mauritianım C. M. $-a$, feuille caulinaire

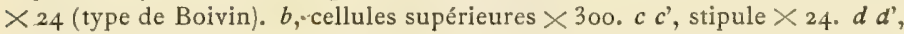
feuille caulinaire $\times 24$. (Maurice, Robillard). $e$, sommet de la feuille $\times 170$. $f$, cellules supérieures $\times 300 . g$, stipule $\times 24 . h$, feuille caulinaire $\times 24$. (var. barbonicum). $i$, cellules supérieures $\times 300 . j$, cellules basilaires juxtacostales $\times 300 . k k^{\prime}$, stipule $\times 24 . l$, sommet de la stipule $\times 170$.

Pl. I 25. - 1. Rhacopilum Ayresii Mitt. $-a$, feuille caulinaire $\times 24 . b$, sommet de la feuille $\times 80 . b^{\prime}$, sommet de la feuille $\times$ r $70, c c^{\prime}$, cellules supérieures $\times 300$. $d$, stipule $\times 24$. -2 . Rhacopilum africanum. $-a$, feuille caulinaire $\times 24 . b$, sommet de la feuille $\times$ r $70 . c$, cellules supérieures $\times 300 . d d^{\prime}$, stipule $\times 24$. $e$, sommet de la stipule $\times 170 .-3$. Rhacopilum madagassum Ren. $-a$, feuille caulinaire $\times 24$ (Ankeranadinika). $b$, cellules supérieures $\times$ $300 . c$, stipule $\times 24 . d d^{\prime}$, feuille caulinaire $\times 24$ (Vinanintelo). $e$, sommet de la feuille $\times 80 . e^{\prime}$, sommet de ia feuille $\times 170 . k k^{\prime}$, cellules supérieures $\times$ $300 . f f^{\prime} f^{\prime \prime}$, stipule $\times 24 . g$, feuille caulinaire $\times 24$ (Fianarantsoa). $h$, sommet de la feuille $\times$ r 70. $i$, cellules supérieures $\times 300 . j$, stipule $\times 24$. 
Pl. 126. - I. Rhacopilum tomentosum Hedw. - $a$, feuille caulinaire $\times$ 24. $b$, sommet de la feuille $\times$ I $70 . c c^{\prime}$, cellules supérieures $\times 300$. $d$, stipule $\times 24$. $e e^{\prime}$, foliole périchétiale $\times 24 . f$, dents externes du péristome $\times 80 . g$, fragment d'anneau $\times 1$ 70. $h$, spores $\times 300$. -2 . Rhacopilum Cardoti Ren. - $a a^{\prime}$. feuille caulinaire $\times 24 . b$, sommet de la feuille $\times 80 . b^{\prime}$, sommet de la feuille $\times$ 170. $c c^{\prime}$, cellules supérieures $\times 300 . d$, cellules basilaires juxtacostales $\times 300 . d^{\prime}$, cellules basilaires juxtamarginales $\times 300$. $e$, stipule $\times 24 . f$, foliole périchétiale $\times 24 . g$, dents externes du péristome $\times 80 . h$, fragment d'anneau $\times 170$. $i$, spores $\times 300$.

PI. 127. - 1. Rhacopilum plicatum R. C. $-a$, feuille caulinaire $\times 24 \cdot b$, sommet de la feuille $\times 1$ 7 o. $c$, cellules supérieures $\times 300$. $d$, stipule $\times 24$. 2. Rhacopilum ellipticum Ren. - $a$, feuille caulinaire $\times 24 . b$, sommet de la feuille $\times 80$. $b^{\prime}$, sommet de la feuille $\times 170 . c c^{\prime}$, cellules supérieures $\times 300$. $d$, cellules marginales supérieures $\times 300$. e, stipule $\times 24 .-3$. Rhacopilum capense C. M. - a $a^{\prime} a^{\prime \prime}$, feuille caulinaire $\times 24$. $b^{\prime} b^{\prime} b^{\prime \prime}$, sommet de la feuille $\times 170 . c c^{\prime}$, cellules supérieures $\times 300 . d$, cellules basilaires juxtacostales $\times 300$. $e e^{\prime} e^{\prime \prime} e^{\prime \prime}$, stipule $\times 24$.

P1. 128. - I. Rhacopilum brevipes C. M. - a feuille caulinaire $\times 24$. $b b^{\prime}$, sommet de la feuille $\times$ I $70 . c$, cellules supérieures $\times 300 . d$, stipule $\times 24$. $e$, dent externe du péristome $\times 80$. -2 . Rhacopilum leptotapes C. M. $-a$, feuille caulinaire $\times 24 . b$, sommet de la feuille $\times$ r $70 . c c^{\prime}$, cellules supérieures $\times 300 . d$, cellules basilaires juxtacostales $\times 300$. e, stipule $\times 24$. $f$, sommet de la stipule $\times$ I70. -3 . Rhacopilum orthocarpoïdes Broth. $-a$, feuille caulinaire $\times 24 . b$, sommet de la feuille $\times 170 . c c^{\prime} c^{\prime \prime}$, cellules supérieures $\times$ $300 . d$, cellules basilaires juxtacostales $\times 300 . e$, stipule $\times 24 . f$, sommet de Ia stipule $\times$ I 70 .

Pl. I29. - I. Rhacopilum spelunce C. M. $-a$, feuille caulinaire $\times 24$. $b b^{\prime}$, sommet de la feuille $\times$ r 7o. $c c^{\prime}$, cellules supérieures $\times 300$. $d$, cellules basilaires juxtacostales $\times 300$. $e$, cellules basilaires juxtamarginales $\times 300$. $f f^{\prime}$, stipule $\times 24 . g$, sommet de la stipule $\times 170 .-2$. Rhacopilum Thomeanum Broth. - $a a^{\prime} a^{\prime \prime}$, feuille caulinaire $\times 24 . b b^{\prime}$, sommet de la feuille $\times \times 70 . c c^{\prime} c^{\prime \prime}$, cellules supérieures $\times 300$. $d$, marge inférieure de la feuille $X$ I $70 . e$, cellules basilaire juxtacostales $\times 300 . f$, cellules basilaires juxtamarginales $\times 300 . \quad g^{\prime \prime} g^{\prime \prime}$, stipule $\times 24$. $h$, sommet de la stipule $\times 170, i$, marge moyenne de la stipule $X$ i 70 .

Pl. 130. - 1. Rhacopilum Schmidii Mitt. $-a$ a', feuille caulinaire $\times 24$ (Ceylon). $b$, sommet de la feuille $\times 80 . c$, cellules juxtamarginales du sommet $\times 300 . d d^{\prime}$, cellules supérieures $\times 300$. $e$, cellules basilaires juxtacostales $\times$ $300 . f$, stipule $\times 24 . g$, feuille caulinaire $\times 24$ (La Réunion). $h$, cellules basilaire juxtacostales $\times 300$. $i$, cellules supérieures $\times 300$. $j$, stipules $\times 24 .-2$. 
Rhacopilopsis Dupuisii R. C. $-a a^{\prime}$, feuille caulinaire $\times 40$. $b$, sommet de la feuille $\times$ i 70 . $c$, cellules supérieures $\times 300$. $d$, cellules moyennes $\times 300$. $e$, cellules inférieures $\times 300 . f f^{\prime}$, cellules des angles et basilaires marginales $\times 300 . g g^{\prime} g^{\prime \prime} g^{\prime \prime}$, stipule $\times 40$. $h$, sommet de la stipule $\times$ i $70 . i$, cellules moyennes $\times 300 . j$, cellules des angles $\times 300 . k$, foliole périchétiale et archégones $\times 40$.

Pl. 131. - Sphagnum Arbogasti Ren. et Card. - a, partie d'une section transversale de la tige $\times 184 . b$, feuille caulinaire $\times 35, c$, tissu basilaire latéral de la même, vu par la face ventrale $\times$ I84. $d$, tissu de la partie supérieure de la même, vu par la face ventrale $\times 184$. $e$, feuille moyenne d'un rameau divergent $\times 35$. $f$, tissu de la partie supérieure de la même, vu par la face ventrale $\times 184 . \mathrm{g}$. partie d'une section transversale de la même vers le milieu $\times 625(1)$. $h$, feuille moyenne d'un rameau pendant $\times 35$.

Pl. 132. - Sphagnum mauritianum Warnst. - $a$, partie d'une section transversale de la tige $\times 184 . b$, feuille caulinaire $\times 35 . c$, tissu basilaire latéral de la même, vu par la face ventrale $\times 184$. $d$, tissu de la partie supérieure de la même, vu par la face ventrale $\times 184$. $e, f$, feuille moyenne d'un rameau divergent $\times 35 . g$, sommet d'une de ces feuilles $\times 82$. $h$, tissu de la partie supérieure de la même, vu par la face dorsale $\times 184$. $i$, partie d'une section transversale de la même, vers le milieu $\times 625$. $j$, feuille moyenne d'un rameau pendant $\times 35$.

Pl. I33. - Sphagnum Rehmanni Warnst. - a, partie de la section transversale de la tige $\times 184 . b$, feuille caulinaire $\times 35 . c$, tissu basilaire latéral de la même, vu par la face ventrale $\times$ r 84 . $d$, tissu de la partie supérieure de la même, vu par la face dorsale $\times 184$. $e, f$, feuilles moyennes d'un rameau divergent $\times 35$. $g$, sommet d'une de ces feuilles $\times 82 . h$, tissu de la partie supérieure de la même, vu par la face ventrale $\times 184$. $i$, le même, vu par la face dorsale $\times 184 \cdot j, k$, parties d'une section transversale de la mềme feuille, vers le milieu $\times 625$.

PI. 134. - Sphagnum obovatum Warnst. - $a$, partie d'une section transversale de la tige $\times 184, b$, feuille caulinaire $\times 35$, c, tissu basilaire latéral de la même, vu par la face ventrale $\times$ r $84 \cdot d$, tissu de la partie supérieure de la même, vu par la face ventrale $\times 184 . e, f$, feuilles moyennes d'un rameau divergent $\times 35$. $g$, sommet d'une de ces feuilles $\times 82$. $h$, tissu de la partie supérieure de la même, vu par la face ventrale $\times$ I 84 . $i$, le même, vu par la face dorsale $\times 184, j$, partie d'une section transversale de la même feuille vers le milieu $\times 625 . k, l$, feuilles moyennes d'un rameau pendant $\times 35$.

(r) Pour toutes les sections transversales des feuilles, la face ventrale se trouve en dessus, la face dorsale en dessous. 
Pl. 135. - Sphagnum Rutenbergii C. Müll. - $a$, partie d'une section transversale de la tige $\times 184 . b, c$, feuilles caulinaires $\times 35, d$, tissu basilaire latéral d'une de ces feuilles, vu par la face ventrale $\times 184 . e$, tissu de la partie supérieure de la même, vu par la face ventrale $\times$ i $84 . f, g$, feuilles moyennes d'un rameau divergent $\times 35$. $h$, sommet d'une de ces feuilles $\times 82$. $i$, tissu de la partie supérieure de la même, vu par la face ventrale $\times$ I $84 . j$, partie d'une section transversale de la même, vers le milieu $\times 625 . k, l$, feuilles moyennes d'un rameau pendant $\times 35$.

PI. 136. - Sphagnum tumidulum Besch. - $a$, partie d'une section transversale de la tige $\times$ r $84, b, c, d$, feuilles caulinaires $\times 35$. $e$, tissu basilaire latéral d'une de ces feuilles, vu par la face ventrale $\times 184 . f$, tissu de la partie supérieure de la même, vu par la face ventrale $\times 184 . g, h$, feuilles moyennes d'un rameau divergent $\times 35$. $i$, sommet d'une de ces feuilles $\times 82 . i$, tissu de la partie supérieure de la même, vu par la face dorsale $\times$ I $84 . k$, portion du même tissu $\times 625 . l$, tissu de la partie supérieure de la même feuille, vu par la face ventrale $\times 184 . m$, partie d'une section transversale de la même, vers le milieu $\times 625$. $n$, o, feuilles moyennes d'un rameau pendant $\times 35$.

P1. 137. - Sphagnum obtusiusculum Lindb. - $a$, partie d'une section transversale de la tige $\times 184 . b, c, d$, feuilles caulinaires $\times 35$. $e$, tissu basilaire latéral d'une de ces feuilles, vu par la face ventrale $\times 184 . f$, partie supérieure de la même, vue par la face ventrale $\times$ i $84 . g, h, i$, feuilles moyennes d'un rameau divergent $\times 35 . j$, sommet d'une de ces feuilles $\times 82, k$, tissu de la partie supérieure de la même, vu par la face ventrale $\times$ i 84 . l, partie d'une section transversale de la même, vers le milieu $\times 625 . m, n$, feuille moyenne d'un rameau pendant $\times 35$.

Pl. I38. - Sphagnum purpureum Sch. - $a$, partie d'une section transversale de la tige $\times$ I $84 . b, c$, feuilles caulinaires $\times 35 . d$, tissu basilaire latéral d'une de ces feuilles, vu par la face ventrale $\times 184 . e$, partie supérieure de la de la même, vue par la face ventrale $\times$ i $84 . f, g, h$, feuilles moyennes d'un rameau divergent $\times 35$. $i$, sommet d'une de ces feuilles $\times 82 . j$, tissu de la partie supérieure de la même, vu par la face ventrale $\times$ I $84 . k$, le même, vu par la face dorsale $\times 184 . l$, partie d'une section transversale de la même feuille, vers le milieu $\times 625 . m, n$, feuille moyenne d'un rameau pendant $\times 35$.

Pl. 139. - Sphagnum Bessoni Warnst. - $a$, partie d'une section transversale de la tige $\times 184 . b, c$, feuilles caulinaires $\times 35 . d$, tissu basilaire latéral d'une de ces feuilles, vu par la face ventrale $\times$ i $84 . e$, partie supérieure de la même feuille, vue par la face dorsale $\times 184 . f, g, h$, feuilles moyennes d'un rameau divergent $\times 35, i$, sommet d'une de ces feuilles $\times 82$. $j$, tissu de la partie supérieure de la même, vu par la face dorsale $\times \times 84 . k$, partie d'une section transversale de la même, vers le milieu $\times 625 . l, m$, feuilles moyennes d'un rameau pendant $\times 35$. 
Pl. 140. - Sphagnum Cardoti Warnst. - a, partie d'une section transversale de la tige $\times 184 . b$, c, feuilles caulinaires $\times 35$. $d$, tissu basilaire latéral d'une de ces feuilles, vu par la face ventrale $\times$ i $84 . e$, partie supérieure de la même, vue par la face ventrale $\times 184 . f, g$, feuilles moyennes d'un rameau divergent $\times 35$. $h$, sommet d'une de ces feuilles $\times 82$, $i$, tissu de la partie supérieure de la même feuille, vue par la face ventrale $\times 184, j$, partie d'une section transversale de la même, vers le milieu $\times 625 . k, l$, feuilles moyennes d'un rameau pendant $\times 35$.

Pl. 141. - Sphagnum pseudo-cuspidatum Warnst. - $a$, partie d'une section transversale de la tige $\times 184 . b, c$, feuilles caulinaires $\times 35$, d, tissu basilaire latéral d'une de ces feuilles, vu par la face ventrale $\times 184$. e, partie supérieure de la même, vue par la face ventrale $\times$ I $84 . f, g, h$, feuilles moyennes d'un rameau divergent $\times 35$. $i$, sommet d'une de ces feuilles $\times 82$. $j$, tissu de la partie supérieure de la même, vu par la face ventrale $\times \mathrm{t} 84 . k$, partie d'une section transversale de la même, vers le milieu $\times 625$.

Pl. I42. - Sphagnum Bernieri Besch, - $a$, partie d'une section transversale de la tige $\times 184 . b, c$, feuilles caulinaires $\times 35 . d$, tissu basilaire latéral d'une de ces feuilles, vu par la face ventrale $\times 184$. e, partie supérieure de la même, vue par la face ventrale $\times 184 . f, g, h$, feuilles moyennes d'un rameau divergent $\times 35 . i$, sommet d'une de ces feuilles $\times 82 . j$, tissu de la partie supérieure de la même, vu par la face ventrale $\times ı 8_{4}, k$, partie d'une section transversale de la même, vers le milieu $\times 625 . l, m, n$, feuilles d'un rameau pendant $\times 35$.

Pl. I43. - Sphagnum ikongoense Warnst. - $a$, partie d'une section transversale de la tige $\times 184 . b, c$, feuilles caulinaires $\times 35 . d$, tissu basilaire latéral d'une de ces feuilles, vu par la face ventrale $\times$ i $84 . e$, partie supérieure de la même, vue par la face ventrale $\times 184 \cdot f, g, h$, feuilles moyennes d'un rameau divergent $\times 35$. $i$, sommet d'une de ces feuilles $\times 82, j$, tissu de la partie supérieure de la même, vu par la face dorsale $\times$ I $84 . k$, partie d'une section transversale de la même, vers le milieu $\times 625 . l, m, n$, feuilles moyennes d'un rameau pendant $\times 35$.

Pl. 144. - Leucobryum Boryanum Besch. $-a$, feuille $\times$ I $7 . \quad b$, sommet d'une feuille $\times 35 . c$, tissu basilaire $\times 184 . d$, leucocystes de la nervure, vers le milieu $\times 184 . e-h$, sections transversales de la feuille à différents niveaux: $e$, à la base, $f$, vers le milieu, $g$, dans la partie supérieure et $h$, au sommet $\times$ 184 .

Pl. 145. - I. Leucobryum Boryanum Besch.(suite). - a, partie d'une section transversale de la tige à l'insertion d'une feuille $\times$ I $84 . b$, partie d'une section longitudinale d'une feuille, dans la partie supérieure $\times 184 .-2$. Lethcobryum Perroti Ren. et Card. - $a, b, c$, feuilles $\times \mathrm{I}_{7}, d, e$, sommet de 
deux feuilles $\times 35 . f, g$, tissu basilaire $\times 184 . h$, leucocystes de la nervure, vers le milieu $\times 184$. $i-l$, sections transversales de la feuille à différents niveaux : $i, j$, à la base, $k$, vers le milieu et $l$, vers le sommet. $\times 184 . m$; partie d'une section transversale de la tige $\times 184$.

P1. I46. - Leucobrrum pseudo-madagassum Card. - a-e, feuilles $\times 17$. $f$, $g$, sommet de deux feuilles $\times 35 . h$, tissu basilaire $\times \mathbf{r} 84, i$, leucocystes de la nervure vers le milieu $\times$ i $84 . j-m$, sections transversales de la feuille à différents niveaux: $j$, à la base, $k$, vers le milieu, $l$, dans le haut et $m$, vers le sommet $\times$ I 84 . $n$, partie d'une section transversale de la tige $\times$ I 84 .

Pl. 147. - Leucobryum Isleanum Besch. $-a-d$, feuilles $\times 17 . e, f$, sommet de deux feuilles $\times 35 . g$, tissu basilaire $\times 184 . h$, $i$, leucocystes de la nervure, vers le milieu $\times 184 \cdot j-n$, sections transversales de la feuille, à différents niveaux: $j, k$, à la base, sur deux feuilles différentes, $l$, vers le milieu, $m, n$, vers le sommet $\times 184.0$, section transversale de la tige $\times 184$.

Pl. 148. - Leucobryum Isleanum Besch. var. molle (C. Müll.) Card. $a-f$, feuilles $\times 17 . g$, sommet d'une feuille $\times 35, h, i$, tissu basilaire $\times 184$. $j, k$, leucocystes de la nervure vers le milieu $\times 184$. $l$ - $t$, sections transversales de la feuille, à différents niveaux et sur différentes feuilles $: l, m, n$, à la base, $o, p$, vers le milieu, $q, r, s, t$, vers le sommet $\times 184 \cdot u, v$, deux sections transversales de la tige, dont la dernière montre le commencement de l'insertion d'une feuille et a le faisceau axile résorbé $\times 184$.

Pl. I49. - 1. Leucobryum acutifolium (Mitt.) Card. $-a, b$, feuilles $\times 17$. $c$, sommet d'une feuille $\times 35$. $d$, tissu basilaire $\times$ I $84 . e$, leucocystes de la nervure, vers le milieu $\times 184 \cdot f$-h , sections transversales de la feuille à différents niveaux: $f$, à la base, $g$, vers le milieu et $h$, ivers le sommet $\times 184 .-2$. Leucobryum cucullatum Broth. var. Rutenbergii (C.M.) Card. $-a-e$, feuilles $\times 17$. $f, g$, deux de ces feuilles $\times 35$. $h$, tissu basilaire $\times r 84$. $i$, leucocystes de la nervure vers le milieu $\times$ i $84 \cdot j-m$, sections transversales de la feuille à différents niveaux: $j, k$, à la base, $l$, vers le milieu et $m$, vers le sommet $\times$ I 84 . $n$, partie d'une section longitudinale de la feuille, vers le sommet $\times \mathrm{I} 84.0$, section transversale de la tige $\times 184 \cdot p, q$, feuilles du type de l'Usambara $\times 17$.

Pl. $150 .-$ Leucobryum comorense C. M. $-a-c$ feuilles $\times 17$. $d$, sommet d'une feuille $\times 35 . e, f$, tissu basilaire $\times 184 . g$, leucocystes de la nervure, vers le milieu $\times 184$. $h-k$, sections transversales de la feuille à différents niveaux: $h$, à la base, $i$, vers le milieu, $j$, dans le haut et $k$, vers le sommet $\times$ 184. $l$, partie d'une section longitudinale de la feuille, dans le haut $\times 184 . m$, section transversale de la tige $\times 184$.

P1. I51. - Leucobryum Sanctee Marice Card. - a-d, feuilles $\times$ i 7.e , sommet d'une feuille $\times 35 . f$, tissu basilaire $\times 184 \cdot g$, leucocystes de la ner- 
vure, vers le milieu $\times 184 . h-p$, sections transversales de la feuille à différents niveaux: $h, i$, à la base, $j, h$, vers le milieu, $l, m, n, o$, dans le haut, $p$, près du sommet $>184$.

Pl. 152. - Leucobryum madagassum Besch. - $a$-d, feuilles $\times 17 . e$, leucocystes de la nervure, vers le milieu $\times 184 \cdot f-k$, sections transversales de la feuille, à différents niveaux: $f$, à la base, $g, h$, vers le milieu, $i$, dans le haut, $j, k$, vers le sommet $\times 184$.

Pl. 153. - 1. Leucobryum madagassum Besch. (suite). - $a$, sommet d'une feuille $\times 35, b$, tissu basilaire $\times 184 . c$, partie d'une section longitudinale de la feuille, vers le sommet $\times$ i 84 . $d$, section transversale de la tige, montrant l'insertion d'une feuille $\times 184 .-2$. Leucobryum marottense Card. $-a-c, j-l$, feuilles $\times$ i $7 . d, m$, sommet de deux feuilles $\times 35 . e, n$, tissu basilaire $\times 184 . f$, $o$, leucocystes de la nervure vers le milieu $\times 184 \cdot g-i, p-r$, sections transversales de la feuille, à différents niveaux: $g, p$, à la base, $h, q$, vers le milieu, $i, r$, vers Ie sommet $\times 184$. (Les figures $2 a-i$ sont prises sur un échantillon original de l'Ochrobrrum Boivini Besch., de Mayotte; les figures $2 j-r$, sur un spécimen récolté par Macé au Mont Kortala, dans la Grande Comore).

Pl. 154. - 1. Leucobryum parvulum Card. - $a-g$, feuilles $\times 17 \cdot h-i$, sommet de deux feuilles $\times 35 . j$, tissu basilaire $\times$ i $84 . k, l, m$, leucocystes de Ia nervure vers le milieu $\times 184 . n-s$, sections transversales de la feuille, à différents niveaux: $n, o, p, q$, dans la partie basilaire, $r$, vers le milieu, $s$, vers le sommet $\times$ i 84 . $t$, partie d'une section longitudinale de la feuille, vers le sommet $\times 184$. $u$, section transversale de la tige $\times 184 .-2$. Leucobryum Galinoni Card. et Par. $-a-c$, feuilles $\times 17 . d$, sommet d'une feuille $\times 35 . e$, tissu basilaire $\times \mathbf{1} 84 . f$ - $h$, sections transversales de la feuille à différents niveaux : $f, g$, dans la partie basilaire, $h$, vers le sommet $\times 184 . i$, partie d'une section longitudinale de la feuille, vers le sommet $\times$ i $84 . j$, section transversale de la tige $\times 184$.

Pl. 155.- I. Leucobryum Galinoni Card.et Par. (suite).-a, leucocystes de la nervure, vers le milieu $\times 184 . b$, section transversale de la feuille, vers le milieu $\times 184 .-2$. Ochrobryum sakalavum Card. et $\mathrm{Par}$. $-a$, tige avec capsule $\times{ }_{7} \cdot b \cdot e$, feuilles $\times{ }^{7} \cdot f$, sommet d'une feuille $\times 35 . g$, tissu basilaire $\times 184$. $h$, leucocystes de la nervure, vers le milieu $\times 184$. $i-o$, sections transversales de la feuille, à différents niveaux : $i$, à la base, $j$ - $l$, dans la partie moyenne, $m$, dans la partie supérieure, $n, o$, près du sommet $\times 184 . p$, section transversale de la tige $\times 184 . q$, capsule et feuilles périchétiales $\times 17 . r$, opercule $\times 17 . s$, coiffe $\times 17 .-3$. Leucophanes Rodriguezii C. Müll. $-a-f$, feuilles $\times 17$. $g$, tissu basilaire $\times 184$.

Pl. 156. - 1. Leucophanes Rodriguezii C. Müll. (suite).-a,leucocystes et 
stéréome marginal vers le milieu $\times \mathrm{I} 84 . b, c$, sommet de deux feuilles, vu par la face dorsale $\times \mathrm{r} 84 . d-i$, sections transversales de la feuille à différents niveaux: $d, e$, à la base, $f, g$, vers le milieu, $h, i$, vers le sommet $\times 184 . j$, partie d'une section longitudinale de la feuille, vers le sommet $\times \mathrm{r} 84 . k$, section transversale de la tige, à l'insertion d'une feuille $\times$ I $84 .-2$. Leucophanes angustifolium Ren. et Card. $-a, b$, feuilles $\times 17$. $c$, tissu basilaire $\times 184$. $d$, leucocystes de la nervure et stéréome marginal, vers le milieu $\times 184 . e$, sommet d'une feuille, vu par la face dorsale $\times 184 \cdot f-j$, sections transversales de la feuille, à différents niveaux : $f$, à la base. $g, h$, vers le milieu, $i-j$, vers le sommet $\times$ i 84 .

Pl. 157. - I. Leucophanes Seychellarum Besch. $-a-c$, feuilles $\times 17 . d$, tissu basilaire $X$ I 84 . $e$, leucocystes de la nervure et stéréome marginal vers le milieu $\times \mathrm{i} 84 . f$, sommet d'une feuille, vu par la face dorsale $\times \mathrm{I} 84 . g-m$, sections transversales de la feuille à différents niveaux: $g, h$, à la base, $i$ - $k$, vers le milieu, $l, m$, vers le sommet $\times 184 .-2$. Leucophanes mayottense Card. $-a, b$, feuilles $\times 17, c$, tissu basilaire $\times 184 . d$, leucocystes de la nervure et stéréôme marginal, vers le milieu $\times 184 . e, f$, sommets de deux feuilles, vus par la face dorsale $\times 184 . g$, section transversale d'une feuille à la base $\times$ I 84 . $h$, section transversale de la tige, montrant le commencement de l'insertion d'une feuille $\times$ I 84 .

P]. I 58. - I. Leucophanes mayottense Card. (suite). $-a$, section transversale de la feuille, vers le milieu, $b, c$, vers le sommet $\times 184$ - -2 . Leucophanes Hildebrandtii. C. Müll. $-a, b$, feuilles $\times$ I $7 . c$, tissu basilaire $\times$ I 84 . $d$, leucocystes de la nervure et stéréôme marginal, vers le milieu $\times 184 . e$, sommet d'une feuille, vu par la face dorsale $\times 184 . f-k$, sections transversales de la feuille, à différents niveaux: $f, g$, à la base, $h, i$, vers le milieu. $j, k$, vers le sommet $\times 184$. $l$, section transversale de la tige $\times 184 .-3$. Leucophanes Renauldi Card. - $a$-e, feuilles $\times 17 . f$, tissu basilaire $\times 184 . g, h$, leucocystes de la nervure et stéréóme marginal, vers le milieu $\times 184 . i, j$, sommet de deux feuilles, vu par la face dorsale $\times 184 . k-q$, sections transversales de la feuille, à différents niveaux : $k, l$, à la base, $m$-o, vers le milieu, $p, q$, vers le sommet $\times 184 . r$, section transversale de la tige $\times 184$.

Pl. I59. - Cardotia heterodictya Besch. - $a-c$, feuilles $\times 17 \cdot d$, sommet d'une feuille $\times 35$. e, tissu basilaire $\times 184, f$, leucocystes de la nervure, vers le milieu $\times 184 . g-k$, sections transversales de la feuille à différents niveaux: $g$, à la base, $h, i$, vers le milieu, $j, k$, vers le sommet $\times 184 . l$, partie d'une section transversale de la tige à l'insertion d'une feuille $\times 184$.

P1. I6o.- - .Cardotia heterodictya Besch. (suite), - a.section transversale de la feuille dans la partie supérieure $\times$ I $84 . b$, partie d'une section longitudinale de la feuille, vers le milieu et $c$, dans le haut $\times 184 .-2$. Cardotia 
heterodictya Besch. var. Boiviniana (Besch.) Card. $-a-h$, feuilles $\times 17 . i$, sommet d'une feuille $\times 35 . j$, tissu basilaire $\times$ I $84 . k, l$, sections transversales de la feuille à la base, $m$, vers le milieu $\times 184 . n$, partie d'une section transversale de la tige $\times 184$. (Les figures $2 a, 2 b$ et $2 k$ représentent une forme de transition entre le type et la var. Boiviniana.)

Pl. 161. - I. Cardotia heterodictya Besch. var. Boiviniana (Besch.) Card. (suite). - $a$, leucocystes de la nervure vers le milieu $\times 184 . b$, partie d'une section transversale de la feuille vers le milieu $\times$ r $84 . c$, section transversale de la feuille vers le sommet $\times 184 .-2$. Cardotia heterodictya Besch. var. microphylla Card. $-a-c$, feuilles $\times 17 . d$, sommet d'une feuille $\times 35$. $e$, tissu basilaire $\times 184 . f$, leucocystes de la nervure vers le milieu $\times 184 \cdot g-j$, sections transversales de la feuille, à différents niveaux: $g, h$, vers la base, $i$, vers le milieu, $j$, vers le sommet $\times 184 .-3$. Octoblepharum albidum $\mathrm{Hedw}$. $-a-d$, feuilles $\times 17 . e$, sommet d'une feuille $\times 35 . f$, tissu basilaire $\times 184$. $g$, leucocystes de la nervure, vers le milieu $\times 184$. $h$, sommet d'une feuille $\times$ 82 .

P1. I62. - Octoblepharum albidum Hedw. (suite). - $a-g$, isections transversales de la feuille, à différents niveaux : $a, b, c$, vers la base, $d, e$, vers le milieu, $f, g$, vers le sommet $\times 184 . h$, partie d'une section longitudinale de la feuille vers le milieu, $i$, au sommet $\times 184 \cdot j$, section transversale de la tige $\times \mathbf{1 8 4}, k$, capsule $\times 17 . l$ fragment du péristome et spores $\times 184$.

Pl. I63. - Octoblepharum africanum (Broth.) Card. - $a-c$; feuilles $\times$ I 7. $d$, sommet d'une feuille $\times 35 . e$, le même $\times 82, f$, partie du même $\times$ I 84 . $g$, tissu basilaire $\times 184 . h$, leucocystes de la nervure vers le milieu $\times 184 . i-o$, sections transversales de la feuille à différents niveaux : $i$, vers la base, $j$, dans la partie inférieure, $k, l$, vers le milieu, $m$, dans la partie supérieure, $n$, $o$, vers le sommet $\times 18+p$, section transversale de la tige $\times 184 \cdot q$, capsule $\times 17 \cdot r$; fragment du péristome et spores $\times$ i 84 . 


\section{PLANCHE I}

a Dicranoloma Menziezii.

$b$ Dicranoloma reflexifolium.

c Dicranoloma subassimile.

d. Dicranoloma subpungens.

e Dicranoloma dichotomum.

$f$ Dicranoloma scopareolum.

g. Dicnemoloma Sieberi.

Coupe transversale de la nervure $>560$. 


\section{Dicranoloma}

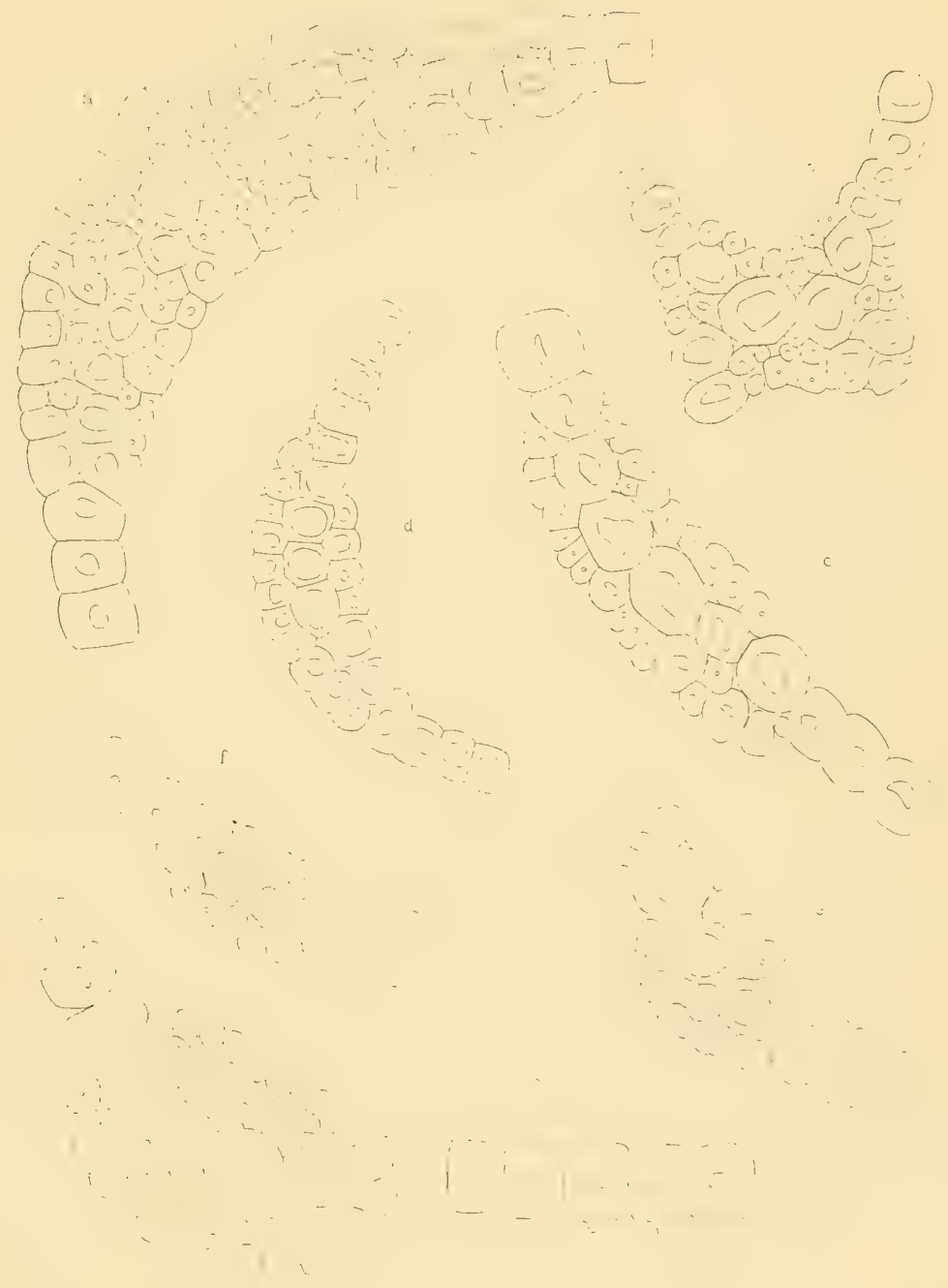

F. Renauld del.

A. diApreval lith. 



\section{PLANCHE II}

\section{Leucoloma ochrobasilare Ren.}

$a a^{\prime} \quad$ Feuille caulinaire $\times 24$.

$b b^{\prime} \quad$ Sommet de la feuille $\times$ i 70 .

c Cellules internes supérieures $\times 3$ oo.

$d d^{\prime} \quad$ Margo hyalin $\times 300$.

\section{Leucoloma leptocladum Ren.}

$a a^{\prime} a "$ Feuille caulinaire $\times 24$.

$b \quad$ Sommet de la feuille $\times 170$.

c Cellules internes $\times 300$.

$d \quad$ Cellules intermédiaires moyennes $\times 300$.

\section{Leucoloma tuberculosum Ren.}

$a a^{\prime} \quad$ Feuille caulinaire $\times 24$.

$b \quad$ Sommet de la feuille $\times 1$ zo.

$c \quad$ Cellules internes supérieures $\times 300$.

$d$ Cellules internes suprabasilaires $\times 300$.

$e \quad$ Saillie interne des papilles $\times 300$.

$f \quad$ Margo hyalin $\times 300$.

$g \quad$ Nervure vers la base $\times$ I 70 . 
Mousses de Madagascar.

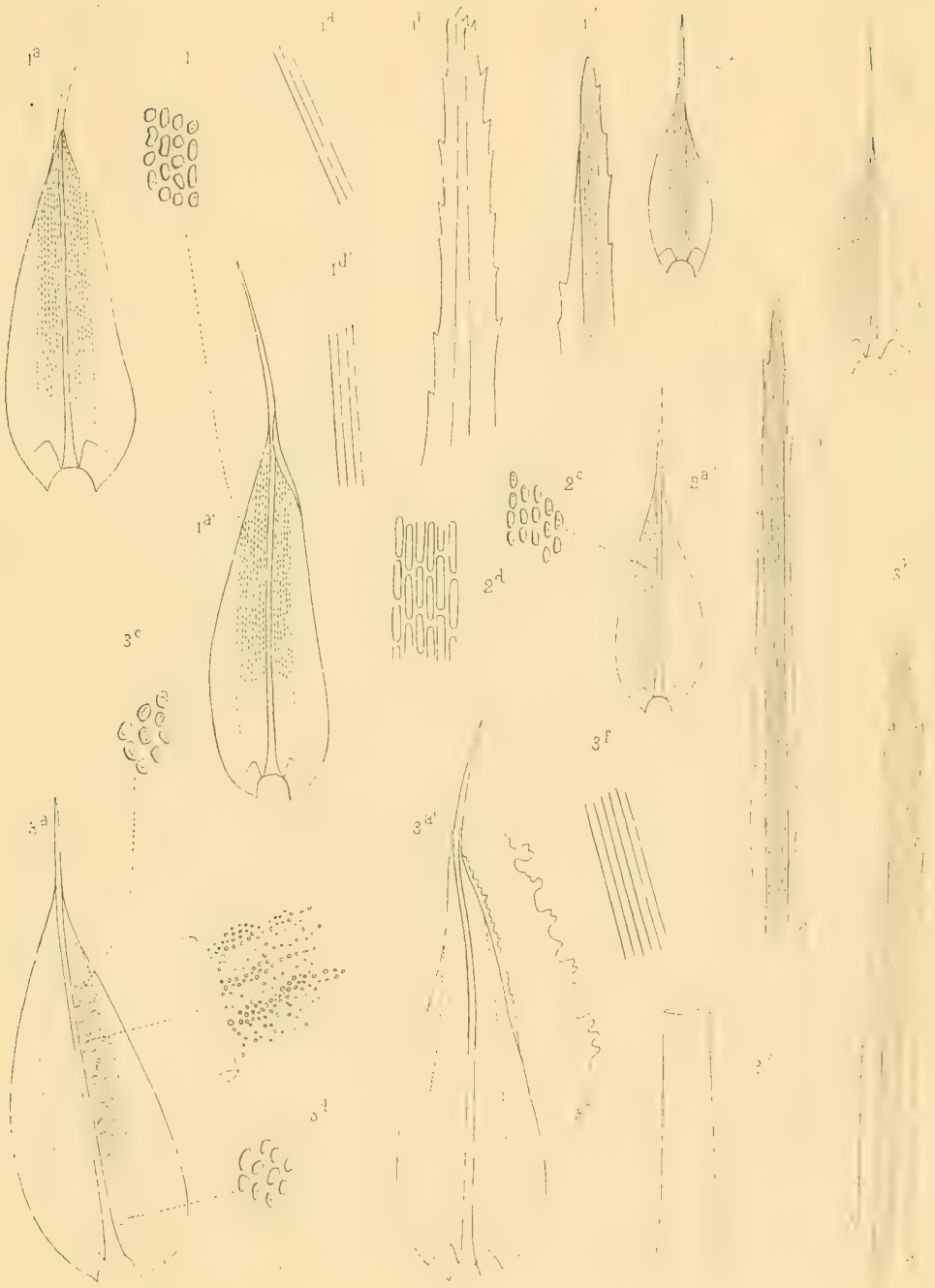

F. Renauld del. 




\section{PLANCHE III}

\section{Leucoloma Levieri Ren.}

$a a^{3} a^{\prime \prime}$ Feuille caulinaire $\times 24$.

$b b^{\prime} \quad$ Sommet de la feuille $\times$ i 7 o.

c Cellules internes supérieures $\times 300$.

$d \quad$ Cellules internes suprabasilaires $\times 300$.

$e \quad$ Tissu membraneux (cellules intermédiaires) $\times 300$.

$f \quad$ Margo hyalin $\times 300$.

$g \quad$ Saillie dorsale des papilles $\times 300$.

h Oreillette $\times 170$.

$i \quad$ Nervure vers la base $\times$ i 70 . 


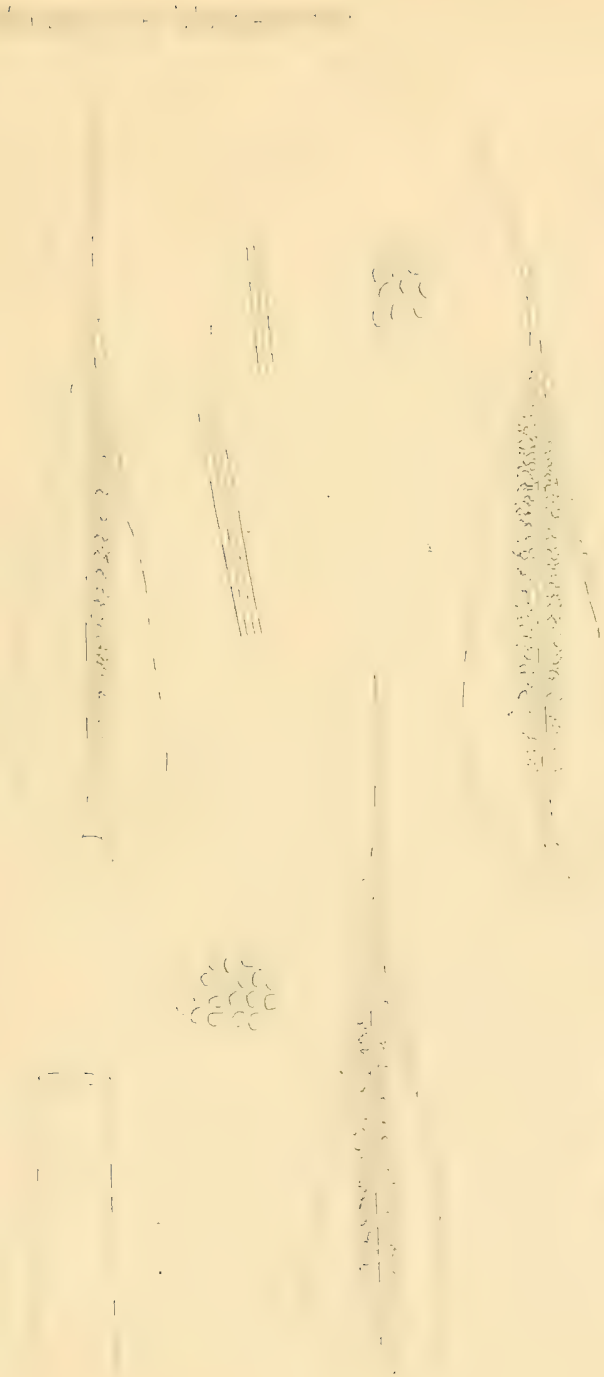

F Renauld del

A. AApr 



\section{PLANCHE IV}

\section{Leucoloma Brotheri Ren.}

$a a^{\prime} a^{\prime \prime}$ Feuille caulinaire $\times 24$.

$b b^{\prime} \quad$ Sommet de la feuille $\times 170$.

c Cellules internes supérieures $\ngtr 300$.

$d$ d' Margo hyalin $>300$.

$e \quad$ Cellules intermédiaires (membraneuses) $\times 300$.

$f \quad$ Oreillette $\times 170$.

2. Leucoloma thraustum Hpe.

a Feuille caulinaire $\times \mathbf{2 4}$.

$b b^{\prime} \quad$ Sommet de la feuille $\times 170$.

c Oreillette $\times 170$.

d Margo hyalin $\times 300$. 


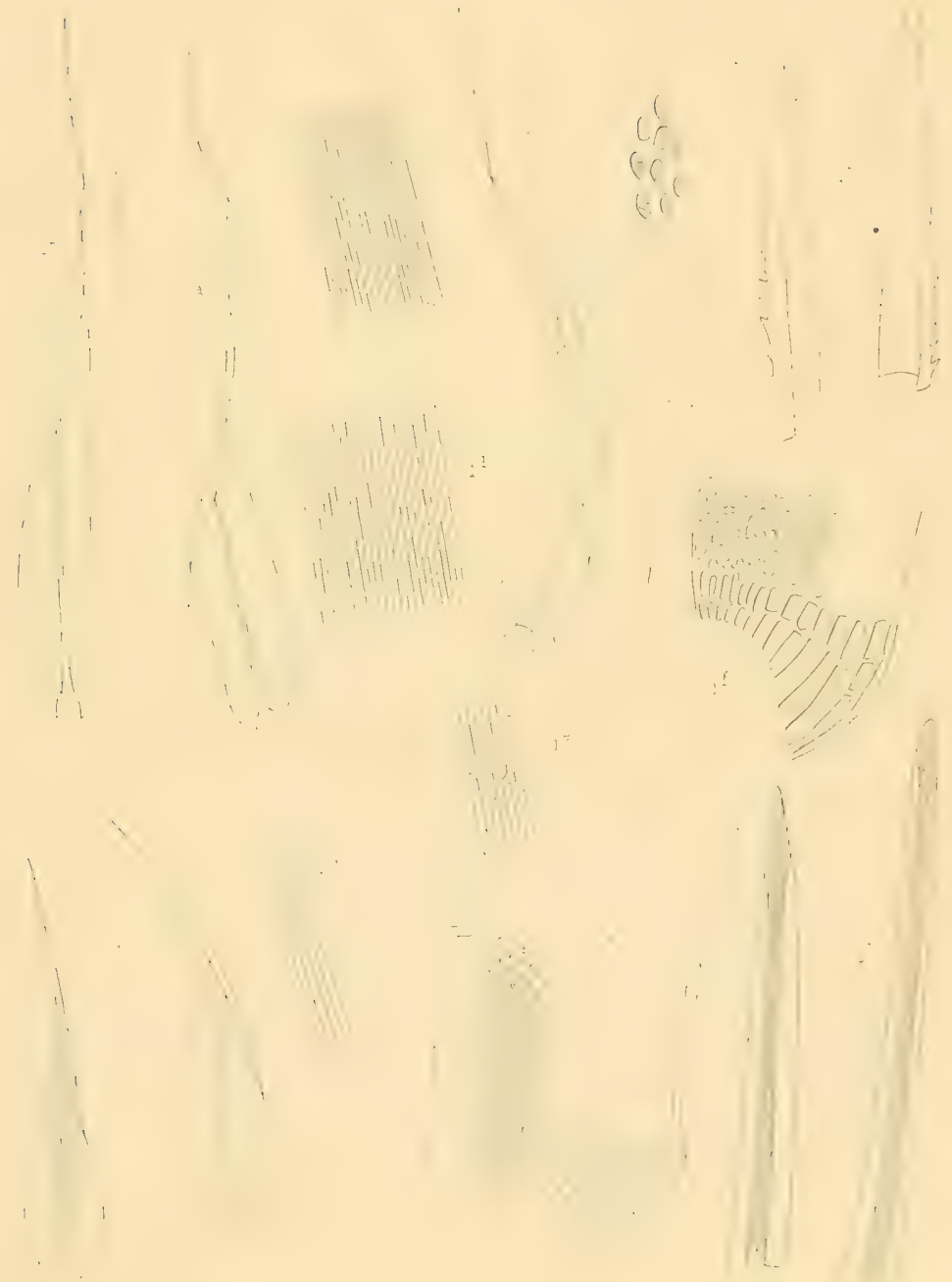






\section{PLANCHE V}

I. Dicranoloma patentifolium Ren. Par.

a Feuille caulinaire $\times 24$.

$b$ Sommet de la feuille $\times 170$.

c Margo hyalin et cellules marginales $\times 300$.

$d$ Cellules un peu au-dessus de la base $\times 3$ oo.

$e$ Oreillette $\times$ i 70 .

$f$ Nervure vers la base $\times$ i 70 .

$g$ Coupe transversale de la nervure $\times 560$. 
Mousses de Madagascar.

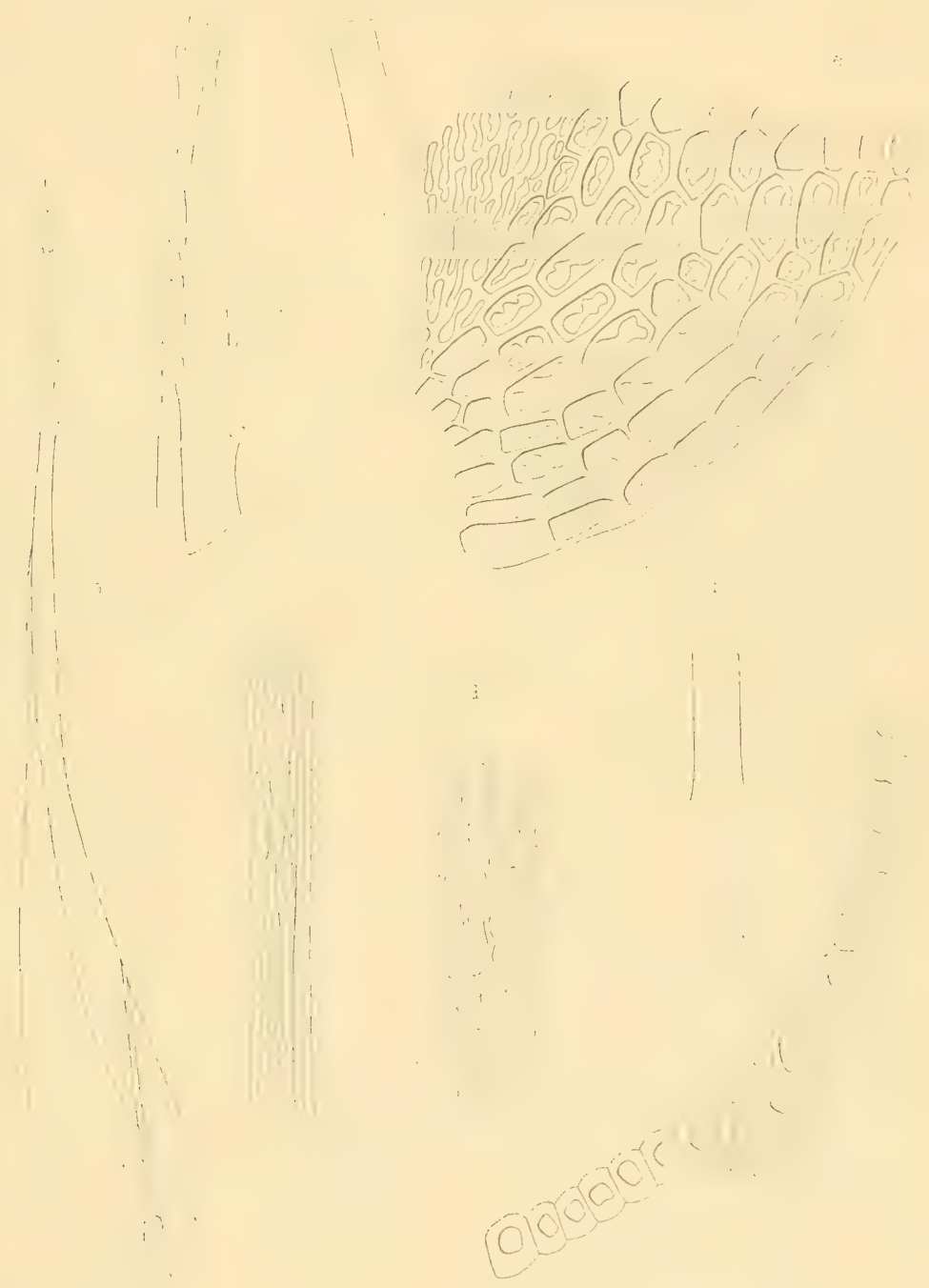

F. Renauld del A. d'Apreval lith 

- 


\section{PLANCHE VI}

\section{Campylopus Gallienii Par.}

a Feuille caulinaire $\times 24$.

$b b^{\prime}$ Sommet de la feuille $\times 170$.

c Cellules basilaires juxtacostales $\times 300$.

$d$ Cellules basilaires juxtamarginales $\times 300$.

e Tissu un peu au-dessus de la base $\times 300$.

g Capsule $\times 24$. 


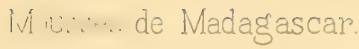

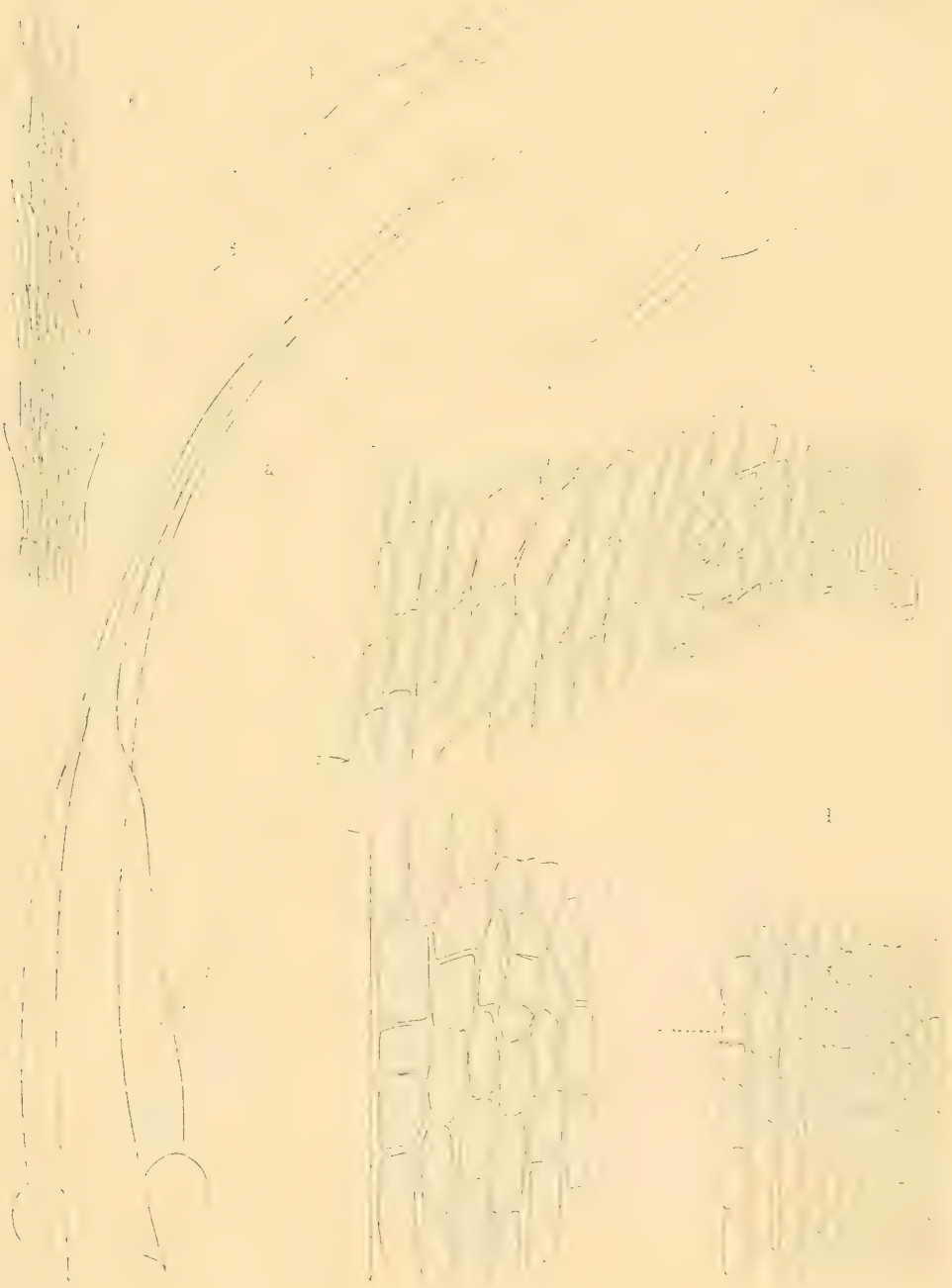

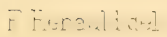





$$
\text { - }
$$




\section{PLANCHE VII}

I. Campylopus Gallienii Par.

Coupe transversale de la nervure $\times 56$ o

2. Campylopus rigens Ren. Card.

Coupe transversale de la nervure $\times 560$.

3. Campylopus subvirescens Ren. Card.

Coupe transversale de la nervure $\times 56$ o.

4. Dicranoloma Blumei.

Coupe transversale de la nervure $>56$ o. 
Miousses de Madagascar

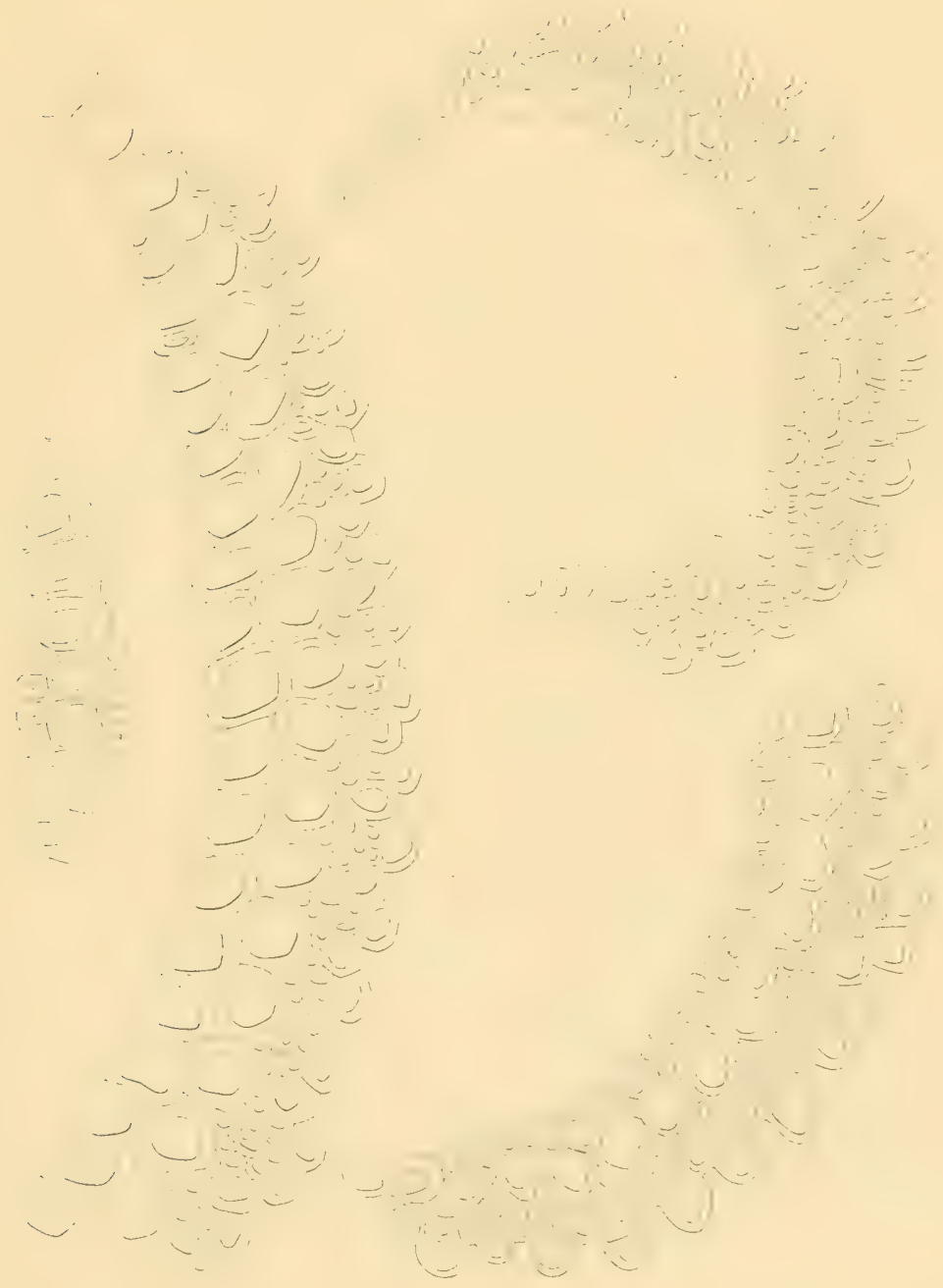

F. Ronzuld del

A dispreval ith 




\section{PLANCHE VIII}

\section{Campylopus crateris Besch.}

a Feuille caulinaire $>24$.

$b$ Tissu basilaire et suprabasilaire $>300$.

c Coupe de la nervure $\chi 560$.

2. Campylopus Eeribaudi Ren. Card.

Coupe de la nervure $\times 560$. 


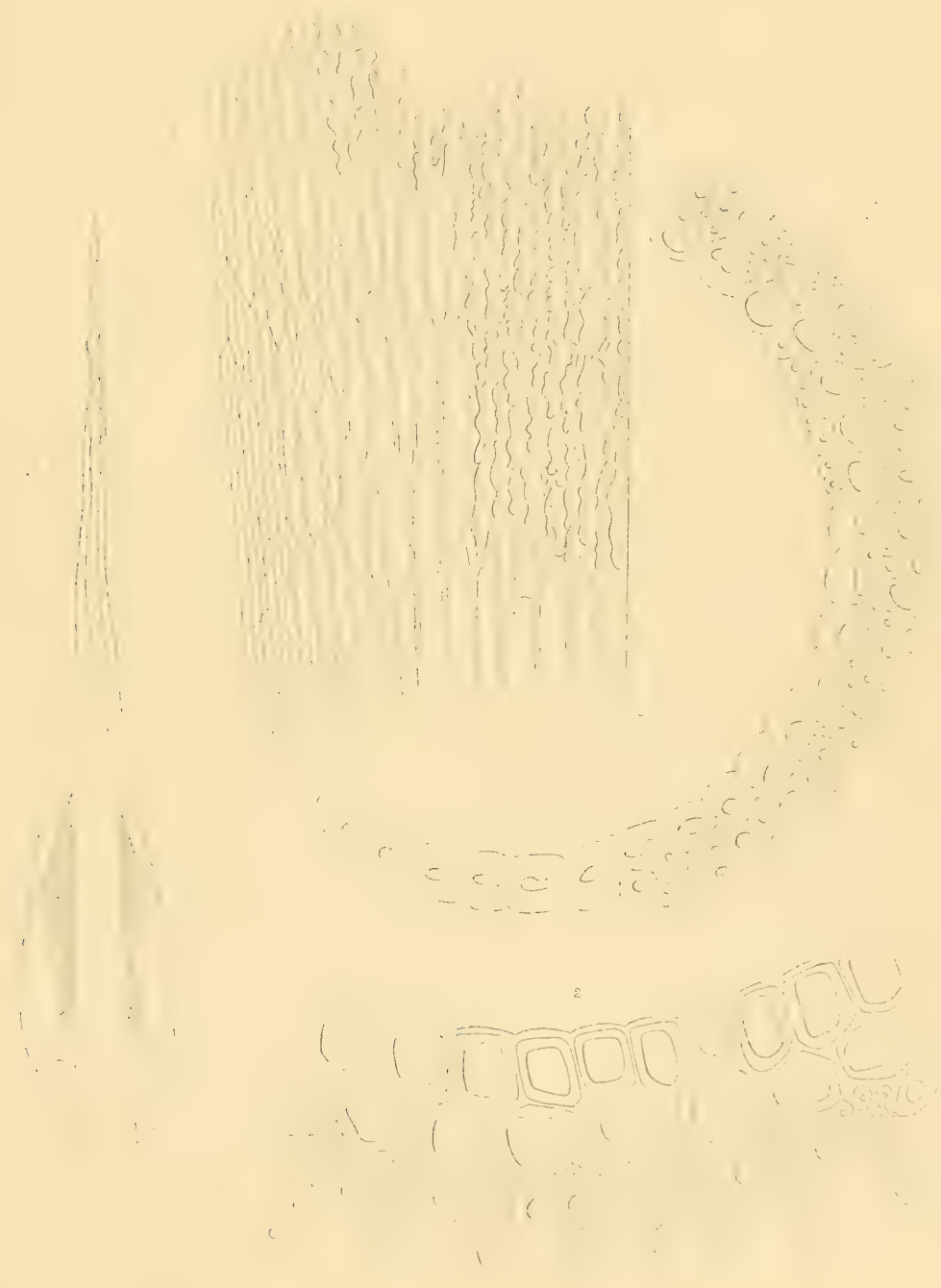

F. Renarid del. 




\section{PLANCHE IX}

\section{Campylopus Orzeszkoanus Ren. Card.}

a Feuille caulinaire $\times 24$.

$b$ Sommet de la feuille $\times 170$.

$d$ Oreillette et tissu basilaire $\times 300$.

$e$ Cellules juxtacostales vers $\mathrm{I} / 4$ inférieur $\times 300$.

$f \quad$ - $\quad$ - vers la moitié $\times 300$.

2. Campylopus comatus Ren. Card.

Coupe transversale de la nervure $\times 560$. 
;....

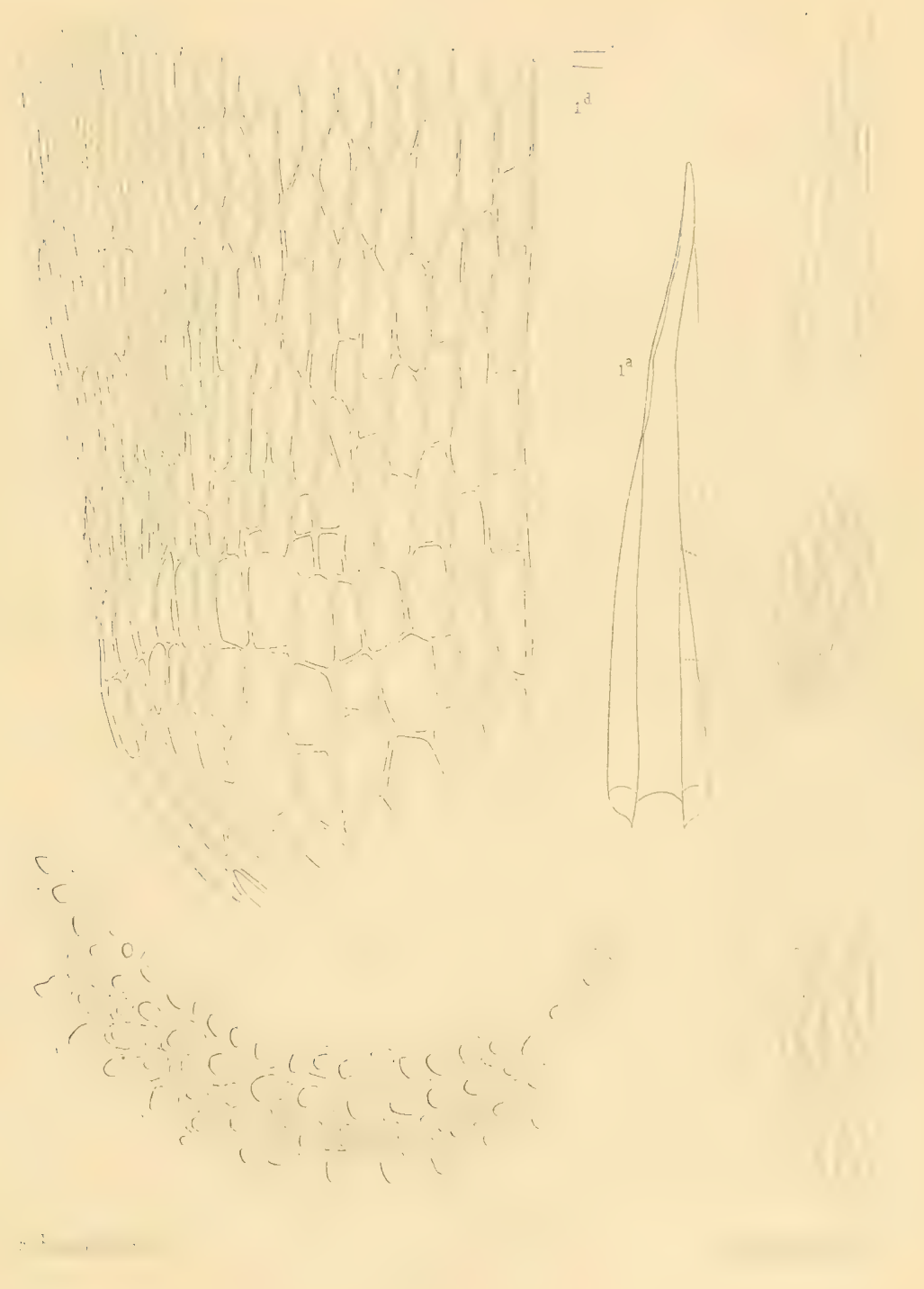






\section{PLANCHE X}

I. Campylopus Orzeszlzoanus Ren. Card.

a Coupe de la nervure à son centre $>560$.

$a$ Coupe de la nervure vers ses bords $>560$.

2. Hyophila Girodi Ren. Card.

a Feuille caulinaire $\times 40$.

$b$ b' Sommet de la feuille $>$ i 70 .

c Portion de la nervure $\Varangle 300$.

$d$ Cellules supérieures $\ngtr 300$.

e capsule $\times 24$.

3. Hypophila usambarica Broth.

a Feuille caulinaire $\chi 40$.

$b$ Sommet de la feuille $>$ i 70 .

4. Hyophila leioneura Ren. Par.

a Feuille caulinaire $\times 40$.

$b$ Sommet de la feuille $\times$ I 70 . 
Mousses de Máadagascar.

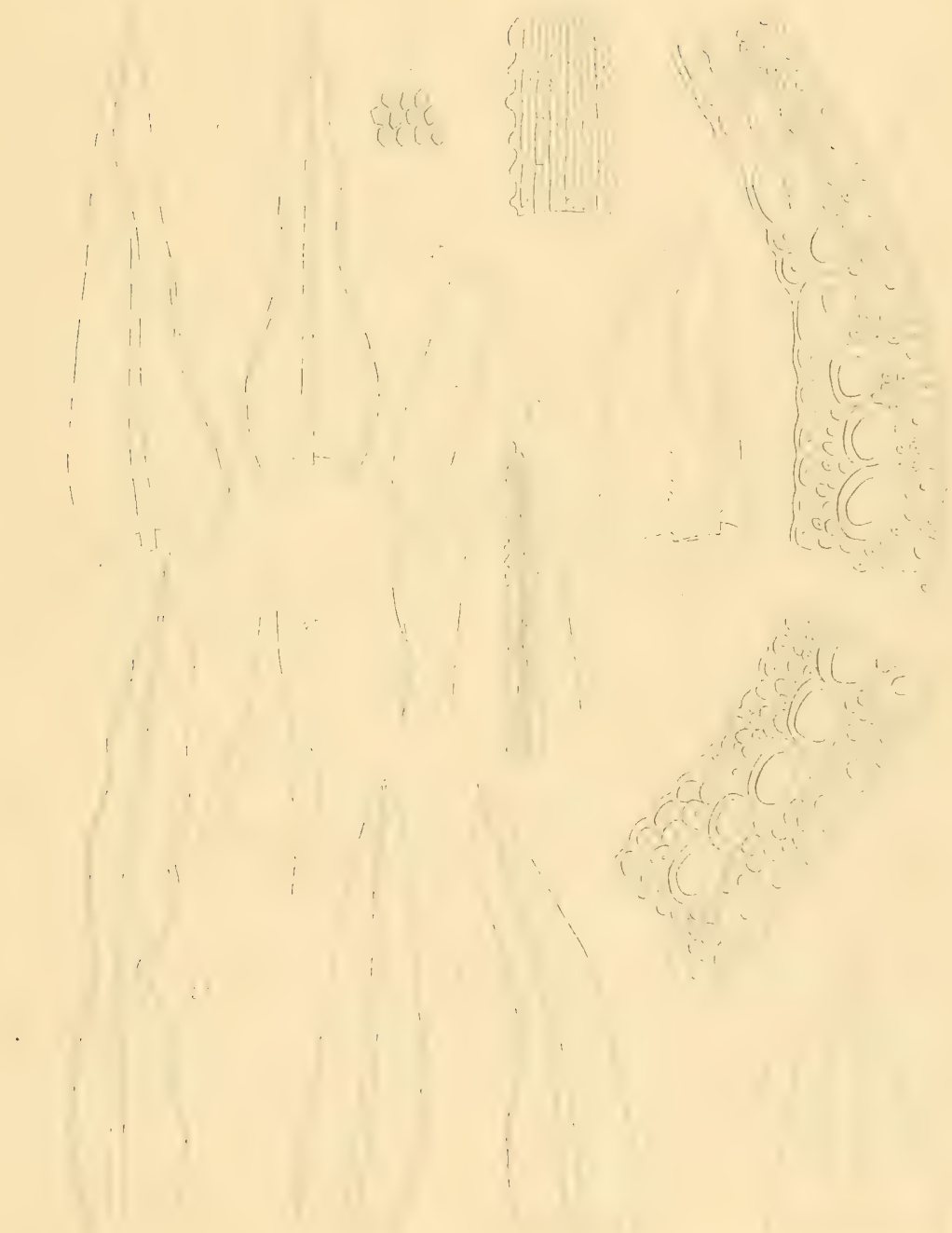

F. Renauld de] 




\section{PLANCHE XI}

I. Hyophila Sakalavensis Ren. Par.

$a$ Feuille caulinaire $\times 40$.

$b$ Sommet de la feuille $\times 170$.

c. Tissu basilaire juxtacostal $\times$ i 70 .

$d$ Cellules moyennes $\times 3$ oo.

2. Hyophila angustifolia Ren. Par.

$a \quad$ Feuille caulinaire $\times 40$.

$b b^{\prime}$ Sommet de la feuille $\times$ i 70 .

3. Barbula (Syntrichia) subrufa Ren. Card.

a Feuille caulinaire $\times 24$.

$b$ Sommet de la feuille $\times$ i 70 . 
Molisses de Madagascar.

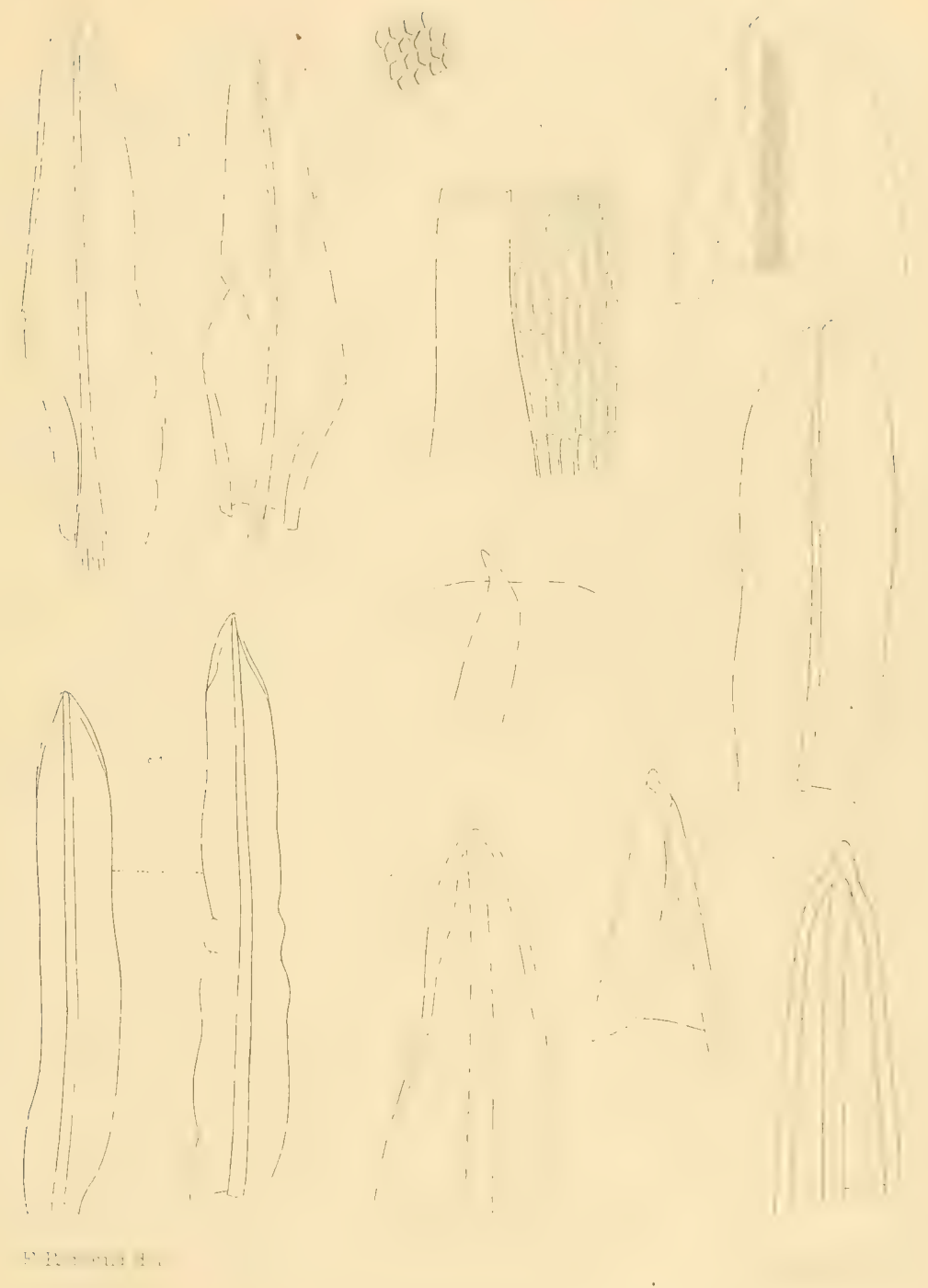






\section{PLANCHE XII}

I. Barbula mucronulata Ren. Card.

a Feuille caulinaire $\times 40$.

$b$ Sommet de la feuille $\times 170$.

c Capsule $\times 24$.

d Spores $\times 300$.

2. Pottia tuberculosa Ren. Par.

a Feuille caulinaire $\times 40$.

$b$ Sommet de la feuille $\times 300$.

c Cellules supérieures $\times 300$.

$d$ Tissu basilaire $\times 170$.

3. Pottia (?) punctulata Ren. Par.

a Feuille caulinaire $\times 40$.

$b$ Cellules subapicales $\times 300$.

c Cellules moyennes $>300$.

$d$ Cellules basilaires marginales $>300$.

$e$ Cellules basilaires juxtacostales $\times 300$. 
Mousse i. Madaga car.
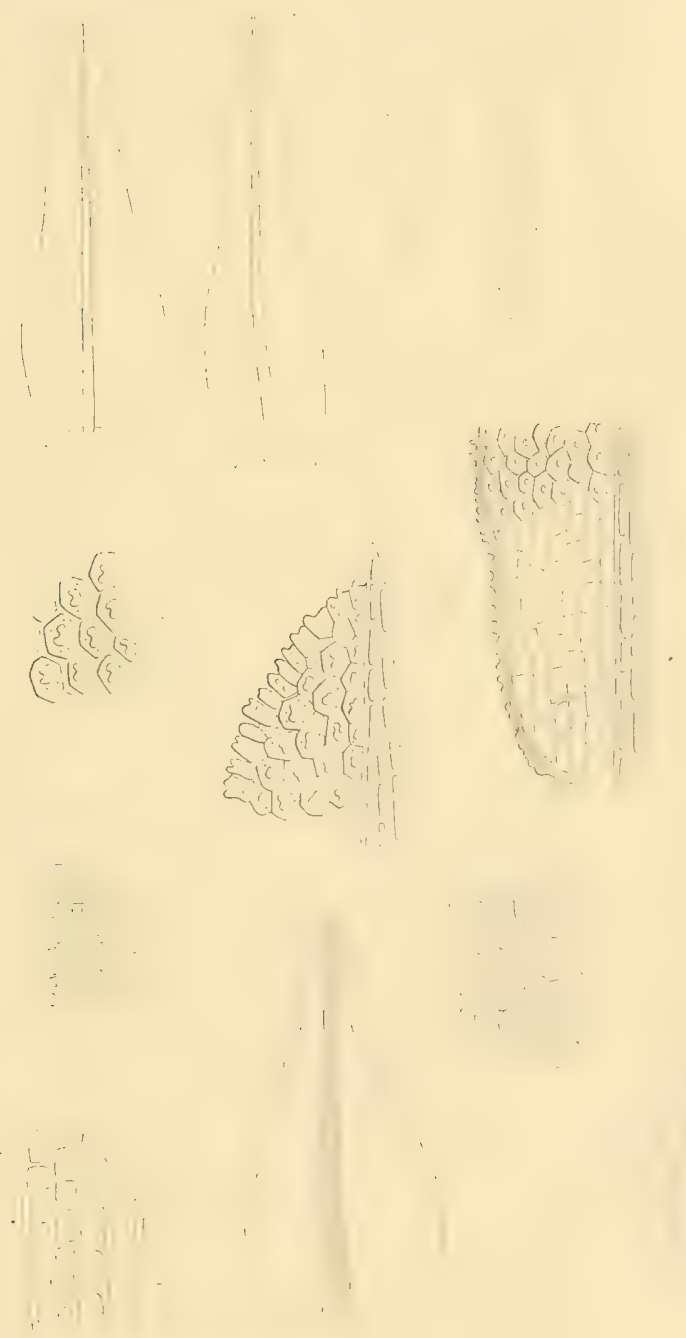

E Fenamle del 




\section{PLANCHE XIII}

I. Schlotheimia PauIi Ren. Card.

$a a^{\prime}$ Feuille caulinaire $\times 40$.

$b$ Sommet de la feuille $\times 80$.

$c$ Cellules subapicales $\times 300$.

$d$ Cellules vers le $1 / 5$ supérieur $\times 300$.

$e$ Cellules juxtacostales moyennes $\times 300$.

$f$ Foliole périchétiale $\times 40$.

$g$ Capsule $\times 24$ (humide).

$i$ Dents de l'exostome $x$ I 70 .

2. Schlotheimia microphylla Besch.

a $a^{\prime}$ Feuille caulinaire $\times 40$.

$b$ Sommet de la feuille $>170$.

c Foliole périchétiale $>40$.

d Capsule $\times 24$.

e Coiffe $\times 24$.

$g$ Dent de l'exostome $>$ i 70 . 
Mousses de Madagascar
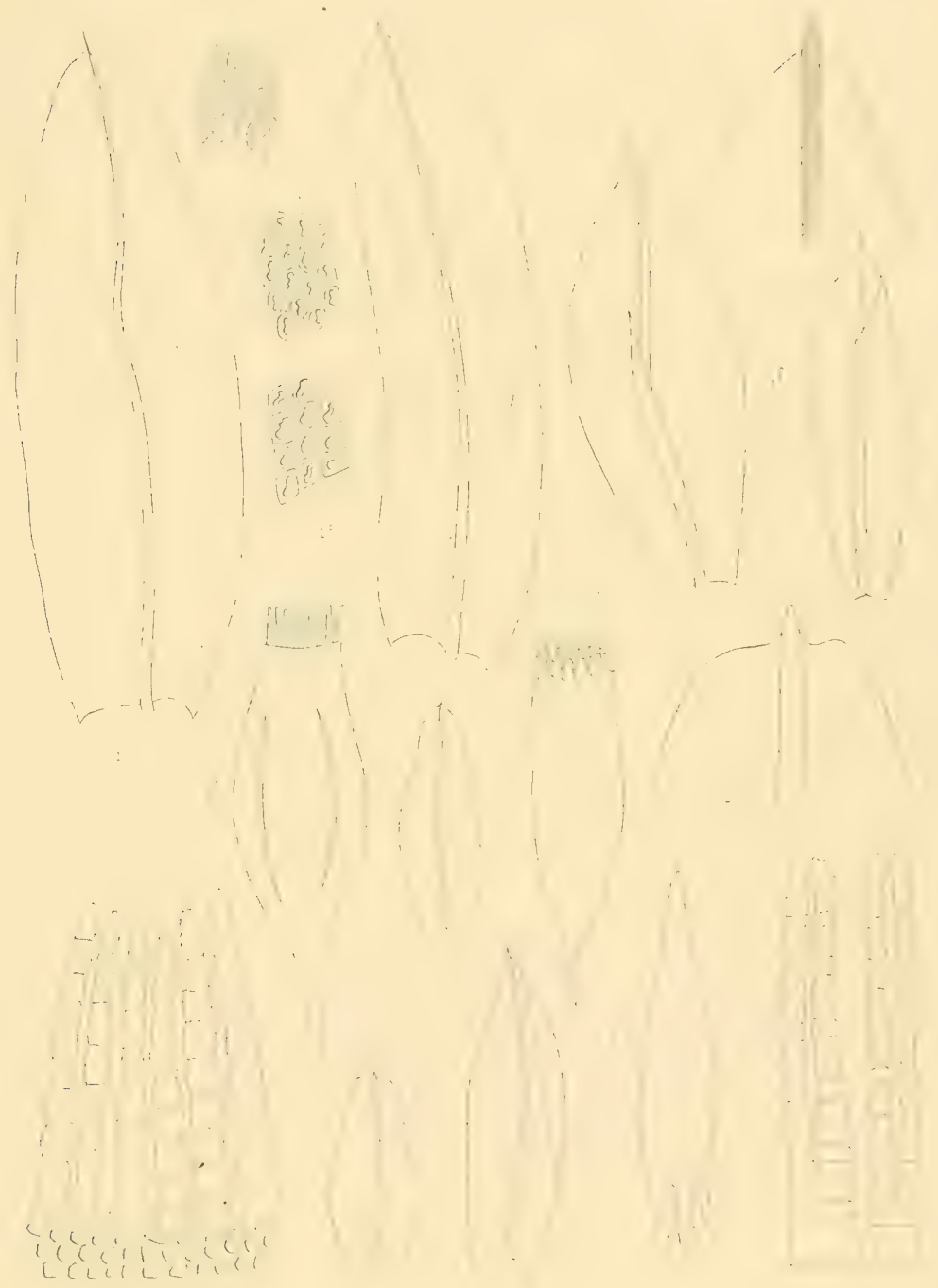

F. Renauld del

A. citpreval litis. 




\section{PLANCHE XIV}

I. Schlotheimia acutifolia Ren. Par.

a Feuille caulinaire $\times 24$.

a" a" a" Feuille caulinaire $\times 40$.

$b \quad$ Cellules supérieures $\times 300$.

c Capsule $\times 24$.

$d$ Coiffe $\times 24$.

2. Hydrogonium Brotheri Ren. Par.

$a a^{\prime} \quad$ Feuille caulinaire $\times 24$.

$b$ Sommet de la feuille $\times 300$. 


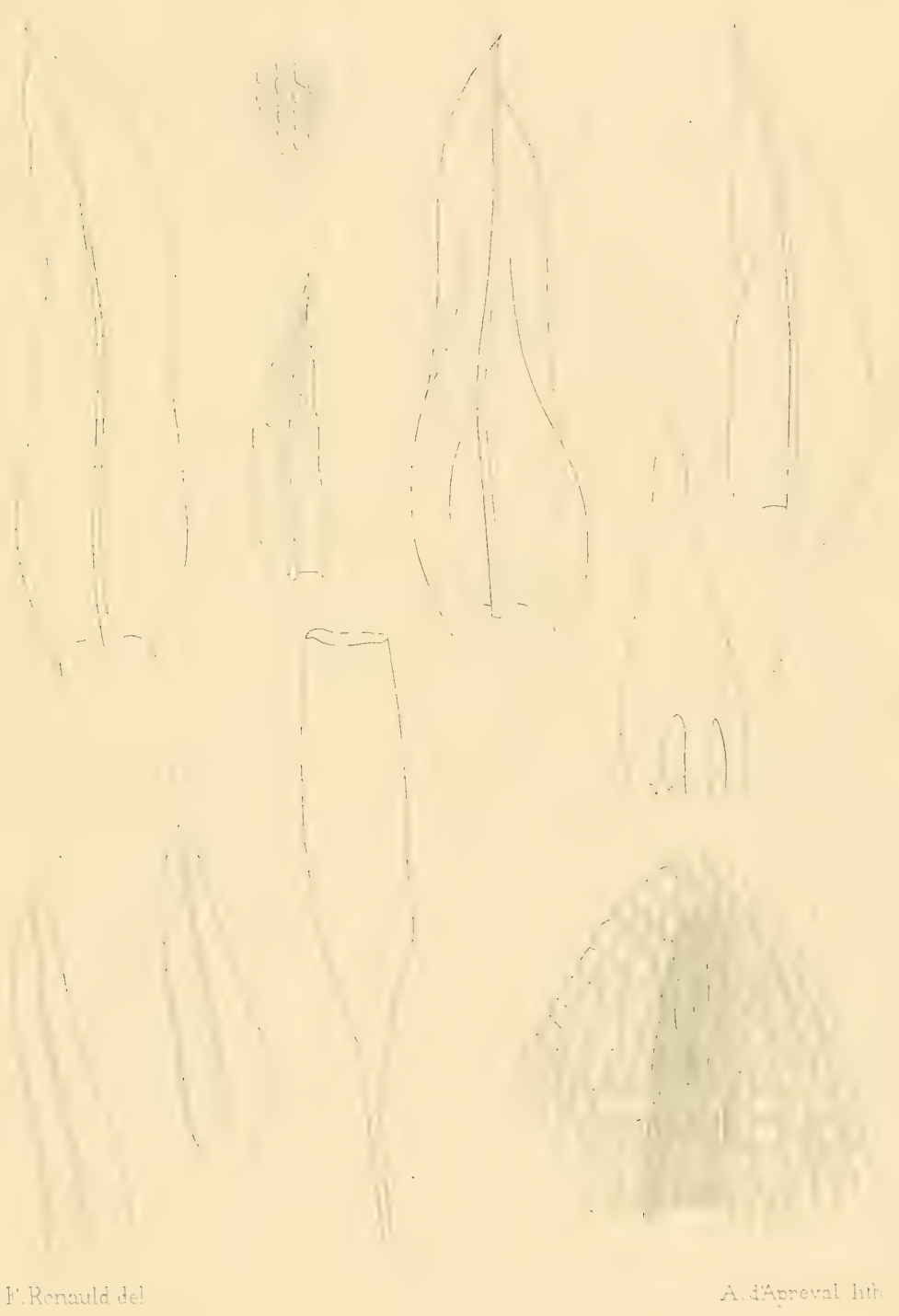






\section{PLANCHE XV}

1. Calymperes Mathiæi Ren. Card.

a Feuille caulinaire $\times 24$.

b Téniole basilaire $\times 300$.

c Téniole suprabasilaire $\times 300$ et cancellines.

d Téniole ventrale $\times 300$.

2. Calymperes erosulum Ren. Par.

a Feuille caulinaire normale $>24$.

a' Feuille caulinaire à sommet proboscidé $\times 24$.

$b$ Sommet d'une feuille normale $\times$ i 70 .

c Marge sous le sommet proboscidé $\times 300$.

$d d^{\prime}$ Téniole vers le milieu de la gaine $\times 300$.

e Téniole ventrale $\times 300$. 
Mousses de Madagascar
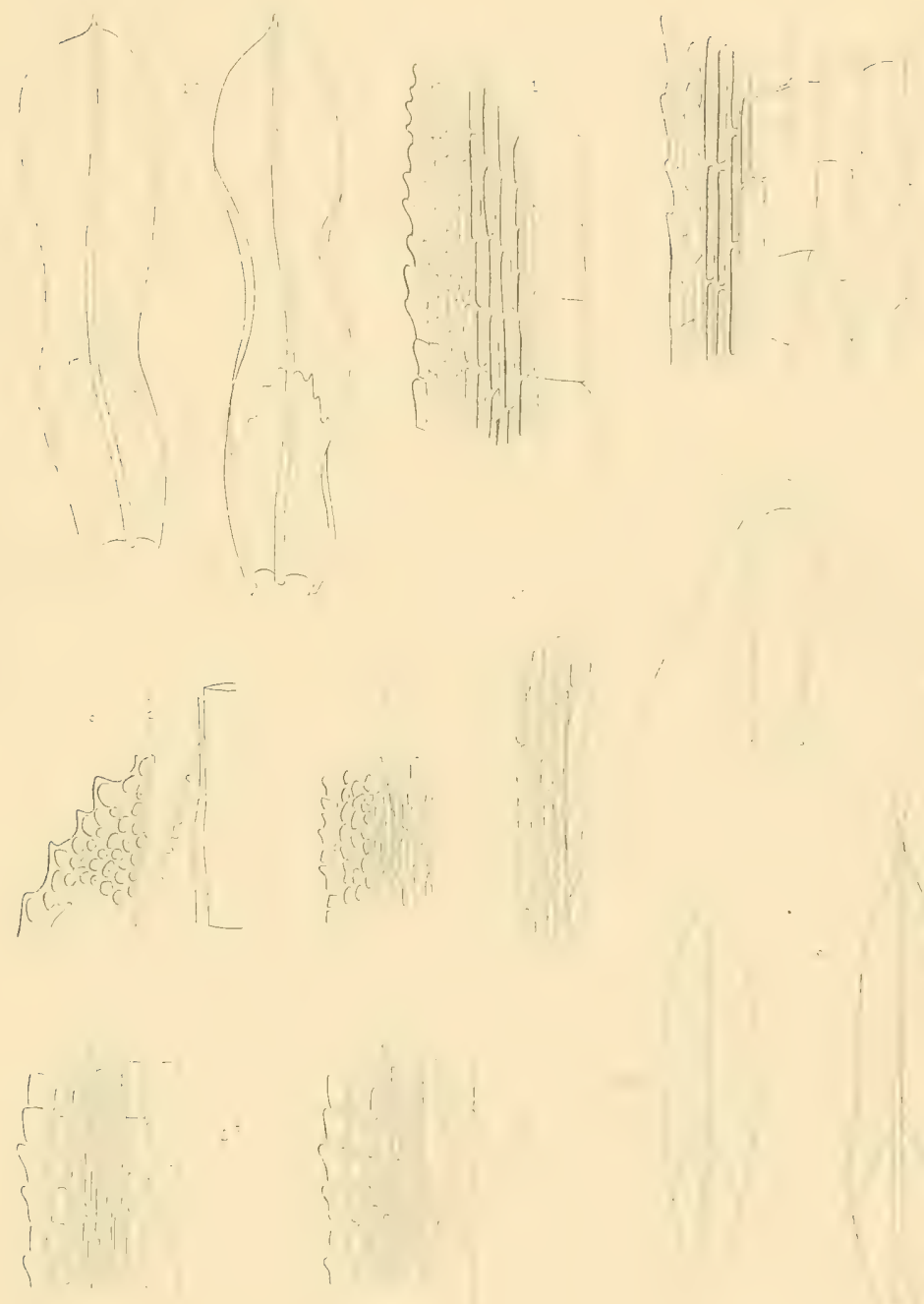

$F \ldots+\cdots$ 




\section{PLANCHE XVI}

I. Erpodium madagassum Ren. Par.

$a a^{\prime} a^{\prime \prime}$ Feuille caulinaire $>24$.

- $b \quad$ Cellules subapicales $>300$.

c Cellules moyennes et inférieurés $\times 300$.

$d$ ' Folioles périchétiales $>24$.

$e \quad$ Capsule $\times 24$.

$f \quad$ Orifice de la capsule montrant l'anneau et les laciniures $\times 170$

2. Garovaglia planifrons Ren. Par.

$a a^{\prime} \quad$ Feuille caulinaire $\times 24$.

a" $\quad$ Feuille caulinaire $>40$.

$b \quad$ Cellules moyennes $>300$.

c Cellules subapicales $\times 300$. 
Mousses de Madagascar.
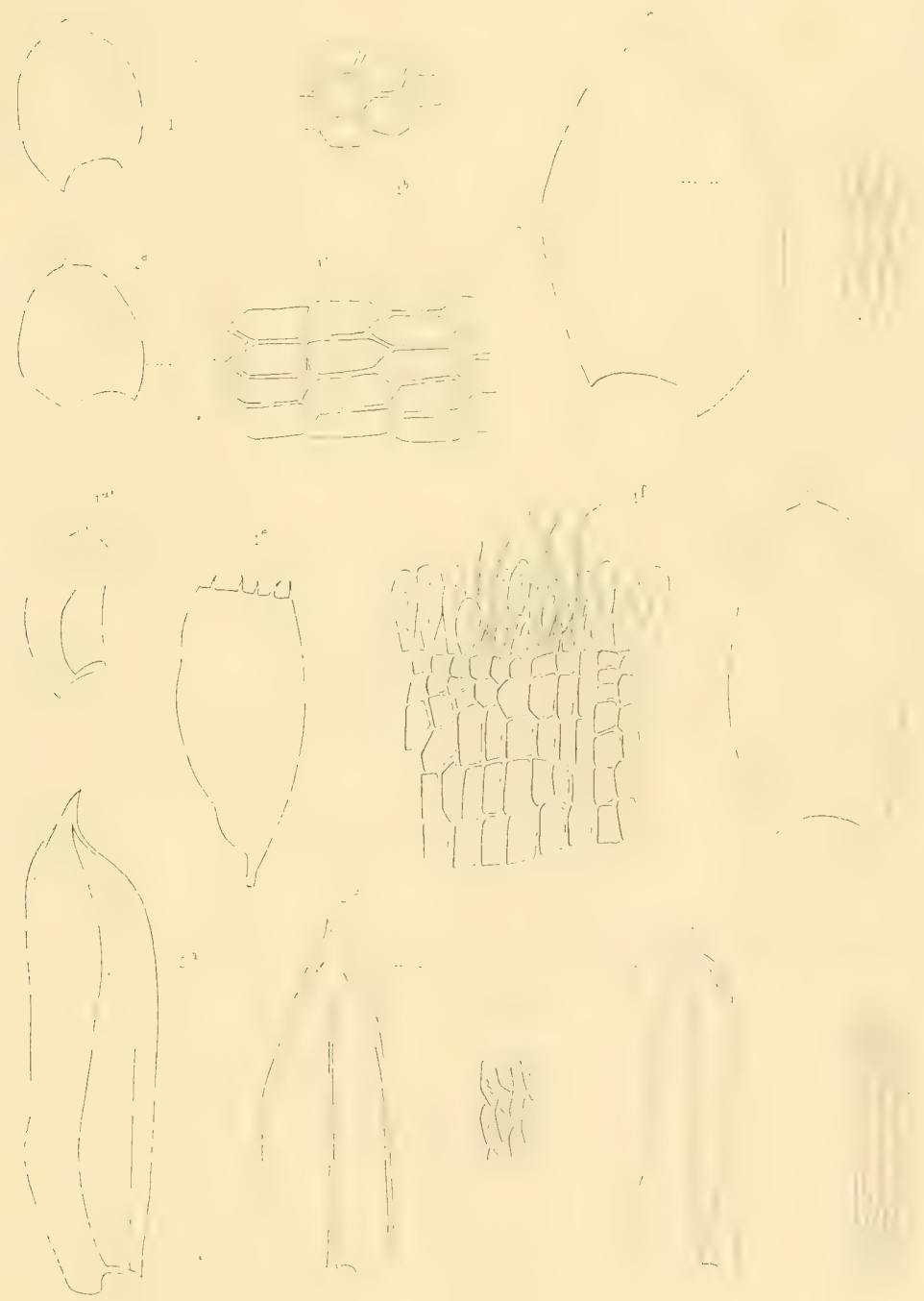

F Renauld del 




\section{PLANCHE XVII}

I. Erythrodontium Lacouturei Ren. Card.

$a a^{\prime}$ Feuille caulinaire $\times 40$.

$b$ Cellules alaires $>$ i 70 .

2. Erythrodontium Engleri Broth.

a a' Feuille caulinaire $\times 40$.

3. Fabronia Lachenaudi Ren. Par.

a Feuille caulinaire de la forme normale $\times 80$.

$a^{\prime}$ Feuille caulinaire de la forme brevifolia $\times 8$ o.

$b$ Sommet de la feuille $\times$ i 70 .

c Marges au-dessous de l'acumen $\times 300$.

d Foliole périchétiale $\times 80$.

$e \quad$ Foliole périgoniale avec une anthéridie $\times 170$.

$f$ Capsule $\times 40$.

$g$ Cellules ondulées de l'exothecium $\times$ r 70 .

lh Spores $>300$. 


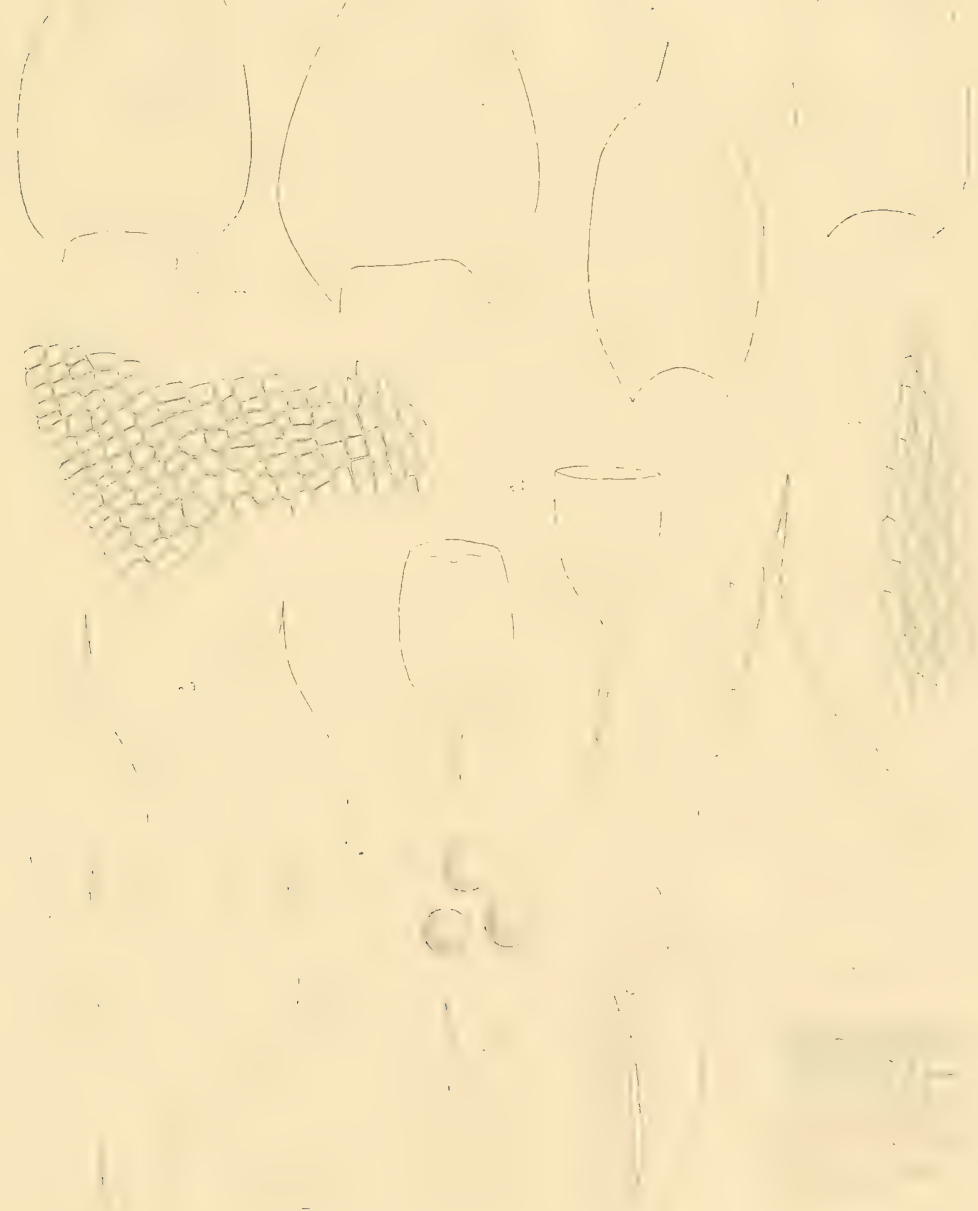

F. Fenald det 




\section{PLANCHE XVIII}

I. Fabronia Villaumii Ren. Card.

$a a^{\prime}$ Feuille caulinaire $>8$ o.

$b \quad$ Marges au-dessoús de l'acumen $\times 300$.

$c$ c' Folioles périchétiales $\times 80$.

$d d^{\prime}$ Capsule $\times 40$ (humide).

$e$ Dent du péristome $\times$ i 70 .

$f f^{\prime}$ Spores $\times 300$.

2. Fabronia Vallis gratiæ Hpe.

a Feuille caulinaire $\times 8$ o.

$b$ Cellules moyennes $\times 3$ oo.

$c c^{\prime}$ Foliole périgoniale $\times 80$.

d Capsule $\times 40$.

e Spores $\times 300$.

3. Fabronia Campenoni Ren. Card.

(Forma sphærocarpa)

a $\quad$ Capsule $\times 40$.

b Spores $\times 300$. 
PI YVII

Let atede

(1)

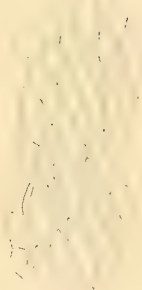

$\therefore 1=$

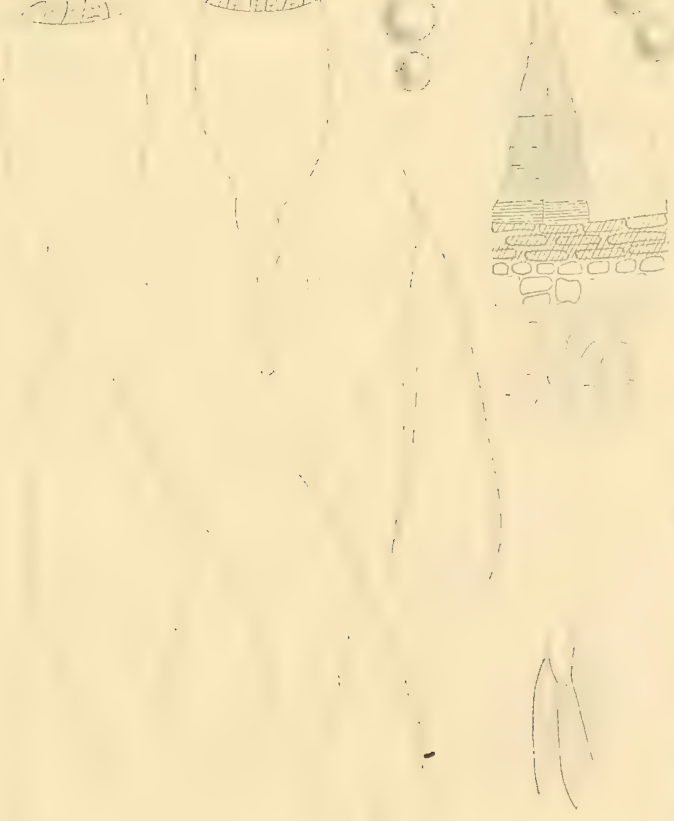

.

10

F Rerauld do: 



$$
-
$$




\section{PLANCHE XIX}

I. Fabronia Garnieri Ren. Par.

a Feuille caulinaire $\times 80$.

$a^{3}$ Sommet de la feuille $\times$ i 70 .

$b$ Marges de la feuille au-dessous de l'acumen $\times 300$.

$c$ Cellules moyennes $\times 300$.

d Foliole périchétiale $>80$.

$e$ Capsule sèche $\times 40$.

$f$ Dent du péristome $\times$ i 70 .

$g$ Spores $\times 300$.

2. Isopterygium Maniæ Ren. Par.

a Feuille caulinaire $>40$.

$b$ Sommet de la feuille $\times$ i 70 .

c Cellules alaires $\times 300$.

d Cellules moyennes $\times 3$ oo.

3. Ispoterygium leiotheca Ren. Card.

a Feuille caulinaire $\times 4$ o.

b Sommet de la feuille $\times 300$.

c Cellules moyennes $\times 300$. 


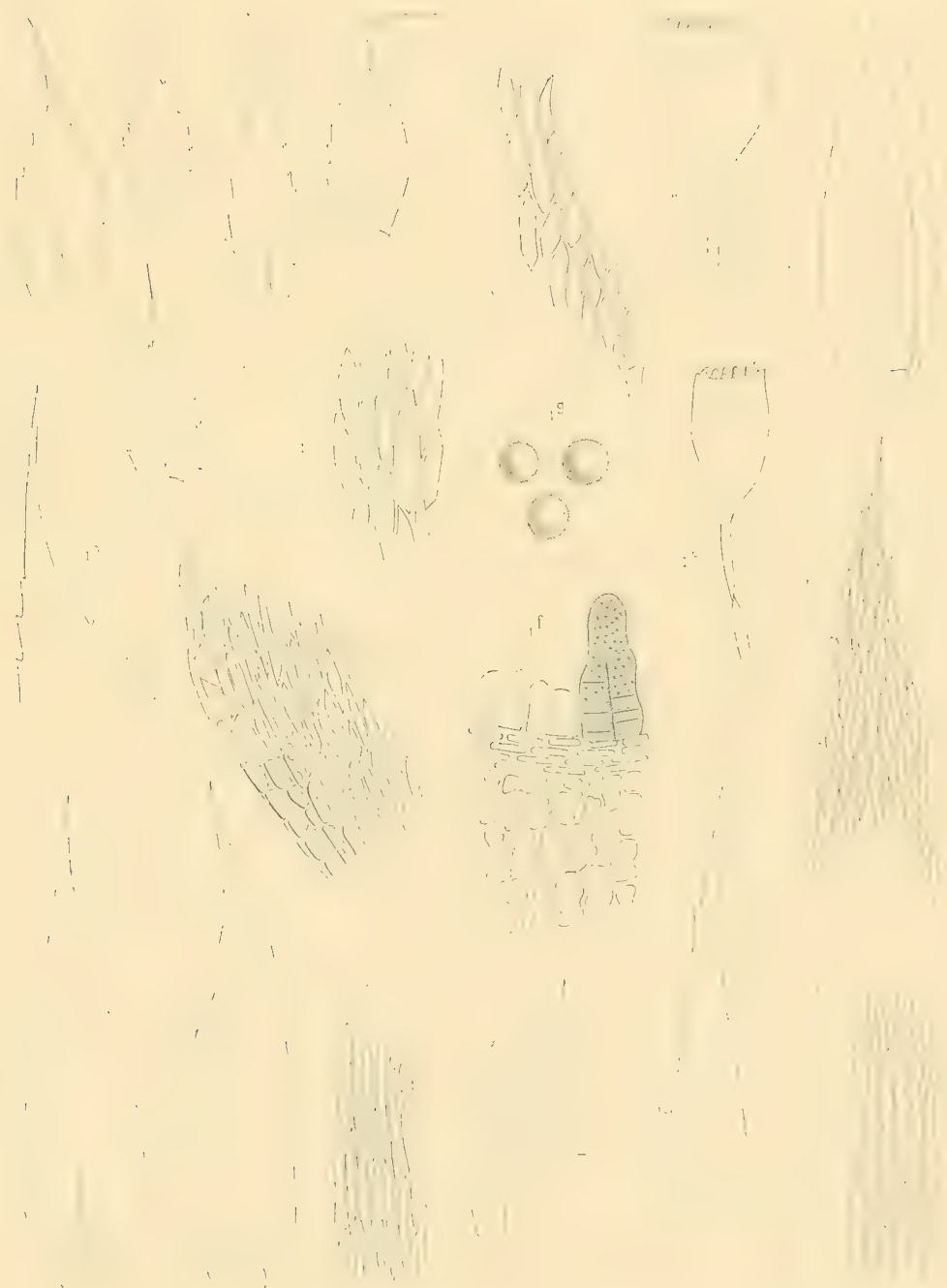





\section{PLANCHE $\mathrm{XX}$}

\section{Isopterygium citrinellum Ren. Card.}

a Feuille caulinaire $\times 40$.

$b$ Sommet de la feuille $\times 300$.

c Aile basilaire $\times 300$.

d Cellules supérieures $\times 300$.

$e$ Cellules moyennes $\times 300$.

2. Lepidopilum subrevolutum Ren. Card.

$a a^{\prime}$ Feuille caulinaire $\times 40$.

b Sommet de la feuille $\times 170$.

c Cellules moyennes $\times 170$.

d Capsule $\times 24$.

$e$ Péristome $\times 170$. 
Mousses de Madagascar

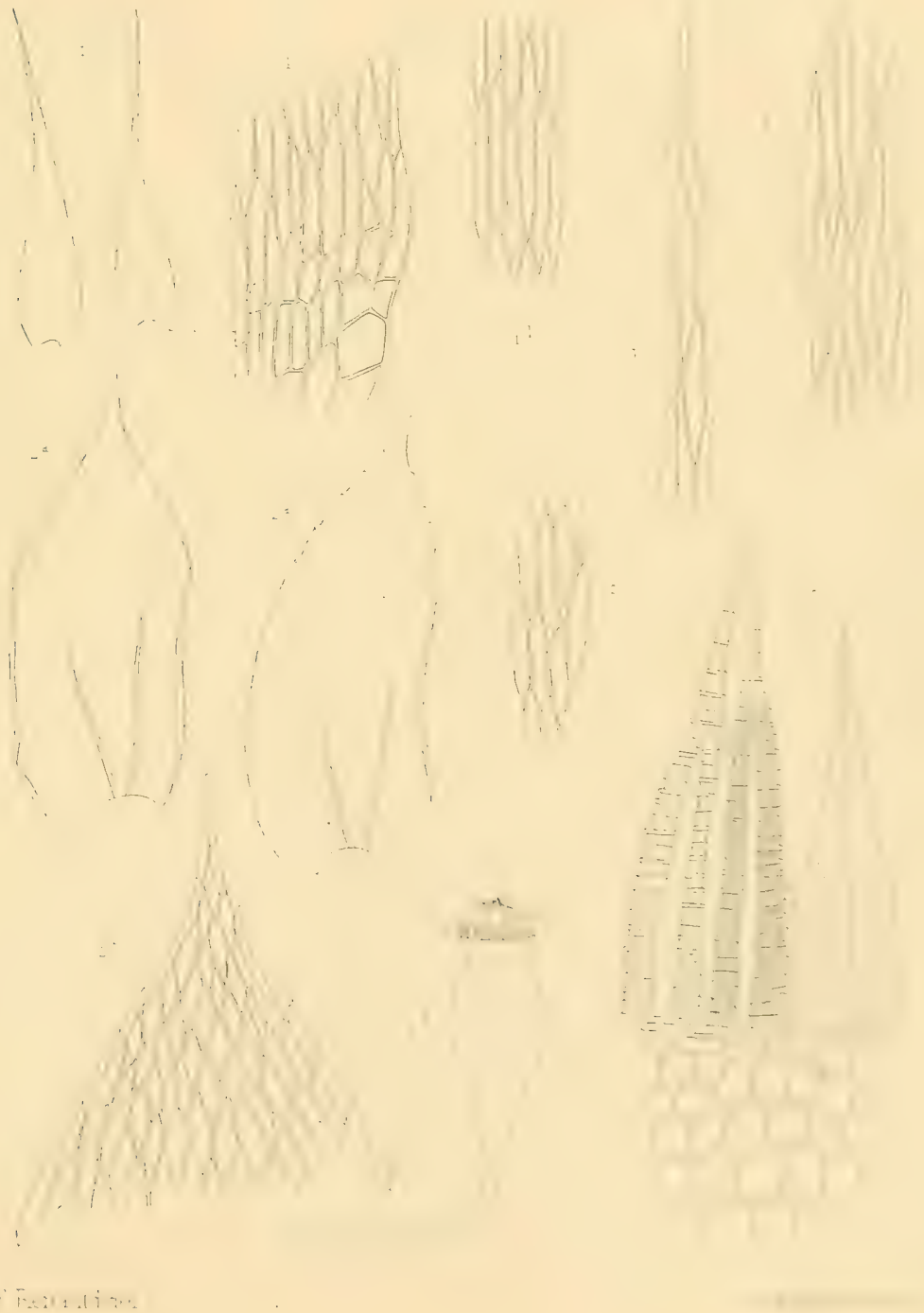






\section{PLANCHE XXI}

\section{Lepidopilum Corbieri.}

$a a^{\prime} \quad$ Feuille caulinaire $\times 40$.

$b \quad$ Sommet de la feuille $\times$ i 70 .

c Cellules moyennes $\times$ i 70 .

$d$ Péristome $\times$ I 80 .

e Spores $\times 300$.

2. Lepidopilum diversifolium Ren. Card.

$a a^{\prime} a^{\prime \prime}$ Feuille caulinaire $\times 40$.

$b \quad$ Cellules marginales $\times 170$.

c Cellules moyennes $\times 170$. 
Musses a a Mrar

$::$

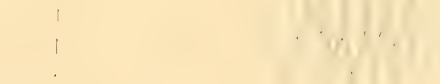

$\vdots$

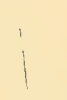

1

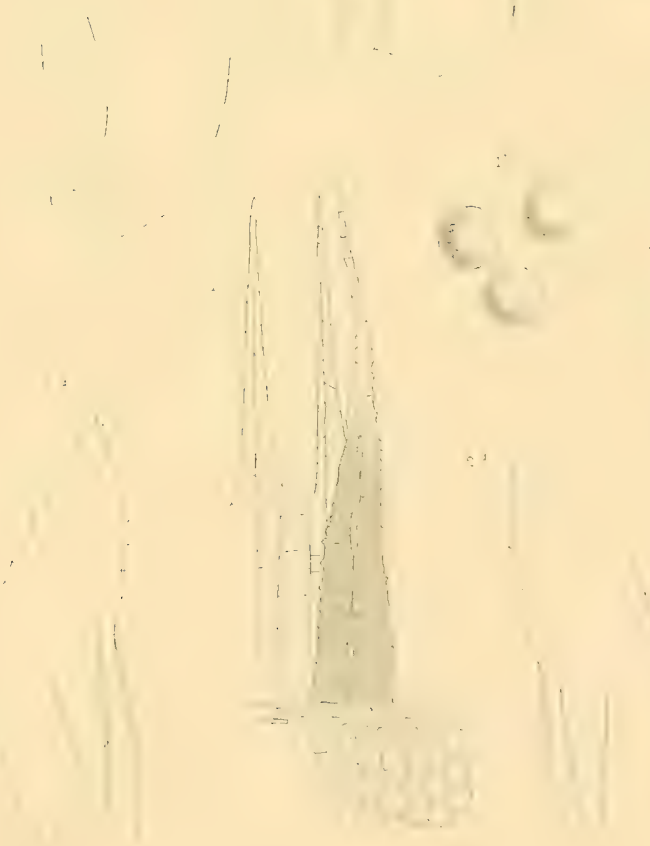

F. Renauld del.

Ad depreval hith 




\section{PLANCHE XXII}

\section{Coleochætium Renauldii Broth.}

a Feuille caulinaire $\times 40$.

$b \quad$ Appendice de l'aile décurrente $\times$ i 70 .

2. Coleochætium subappendiculatum Broth.

$a a^{\prime} a^{3}$ Feuille caulinaire $\times 40$.

b Sommet de la feuille $\times 170$.

$c c^{\prime} \quad$ Aile hyaline décurrente $\times$ I 70 .

3. Pseuloleskea obtusiuscula Ren. Card.

$a a^{\prime} \quad$ Feuille caulinaire $\times 40$.

$b \quad$ Sommet de la feuille $\times 300$.

c Cellules moyennes $\times 300$.

4. Pseudoleskea claviramea C. MIüll.

a Feuille caulinaire $\times 40$.

$b \quad$ Cellules moyennes $\times 300$.

\section{Dicranoloma dicarpum.}

a Tissu foliaire vers le I/ 3 supérieur $\times 300$. 
lviousses de . a. SC.

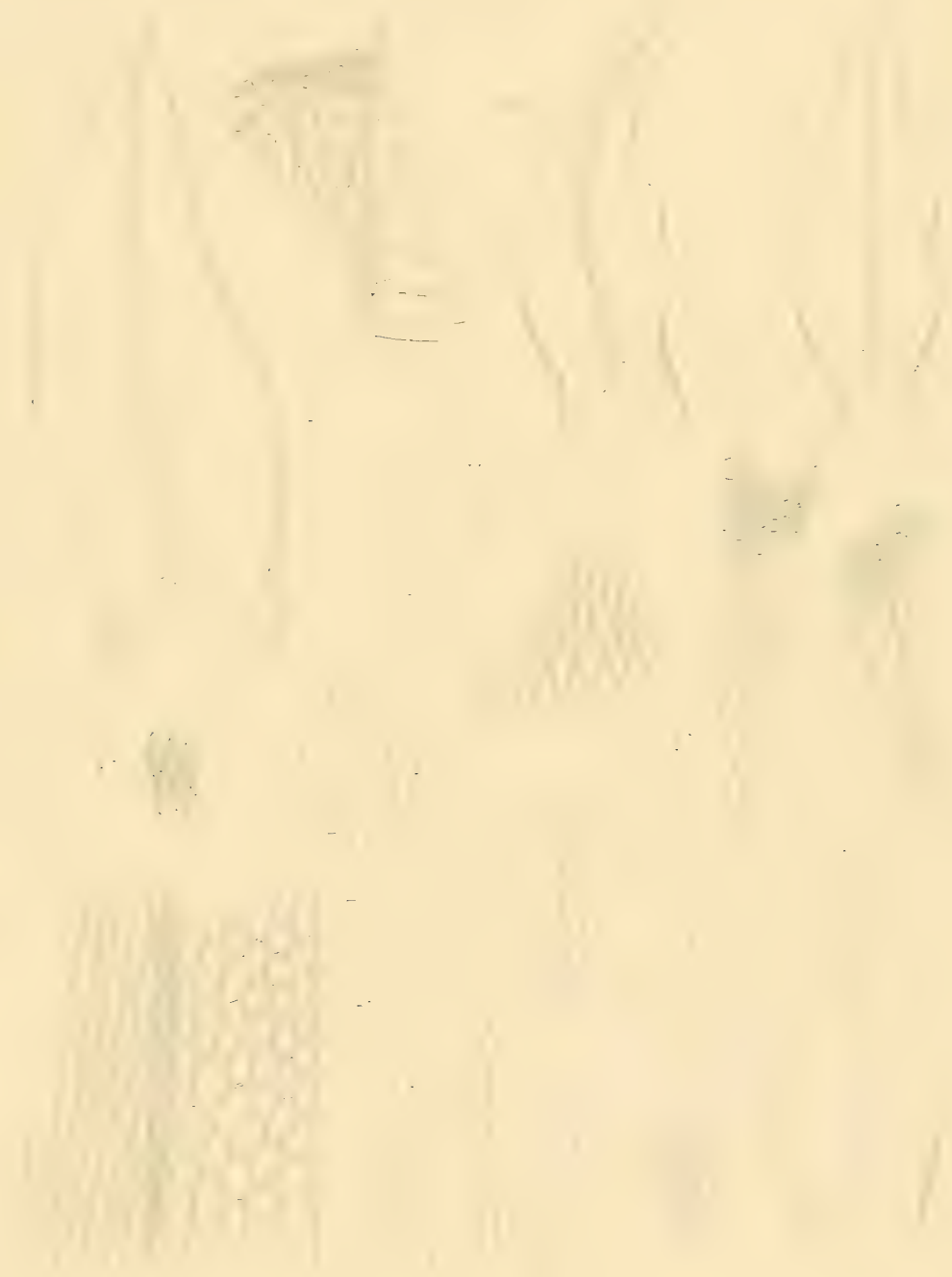

F.Renauld de! 




\section{PLANCHE XXIII}

\section{Enthosthodon Renauldii Thériot.}

a Plante, grandeur naturelle.

$b b^{\prime}$ Feuilles moyennes $>22$.

$c$ Tissu marginal vers le $1 / 3$ inférieur $\times 120$.

d Tissu de l'acumen $\times 120$.

$e \quad$ Capsule sèche $\times 22$.

$e^{\prime} \quad$ Capsule humide $\chi 22$.

2. Epipterygium diversifolium Ren. Par.

a Feuilles supérieures $>40$.

$b b^{\prime}$ Feuilles inférieures $>4$ o.

c Tissu marginal $\times 300$. 
Mousses de Madagascar.

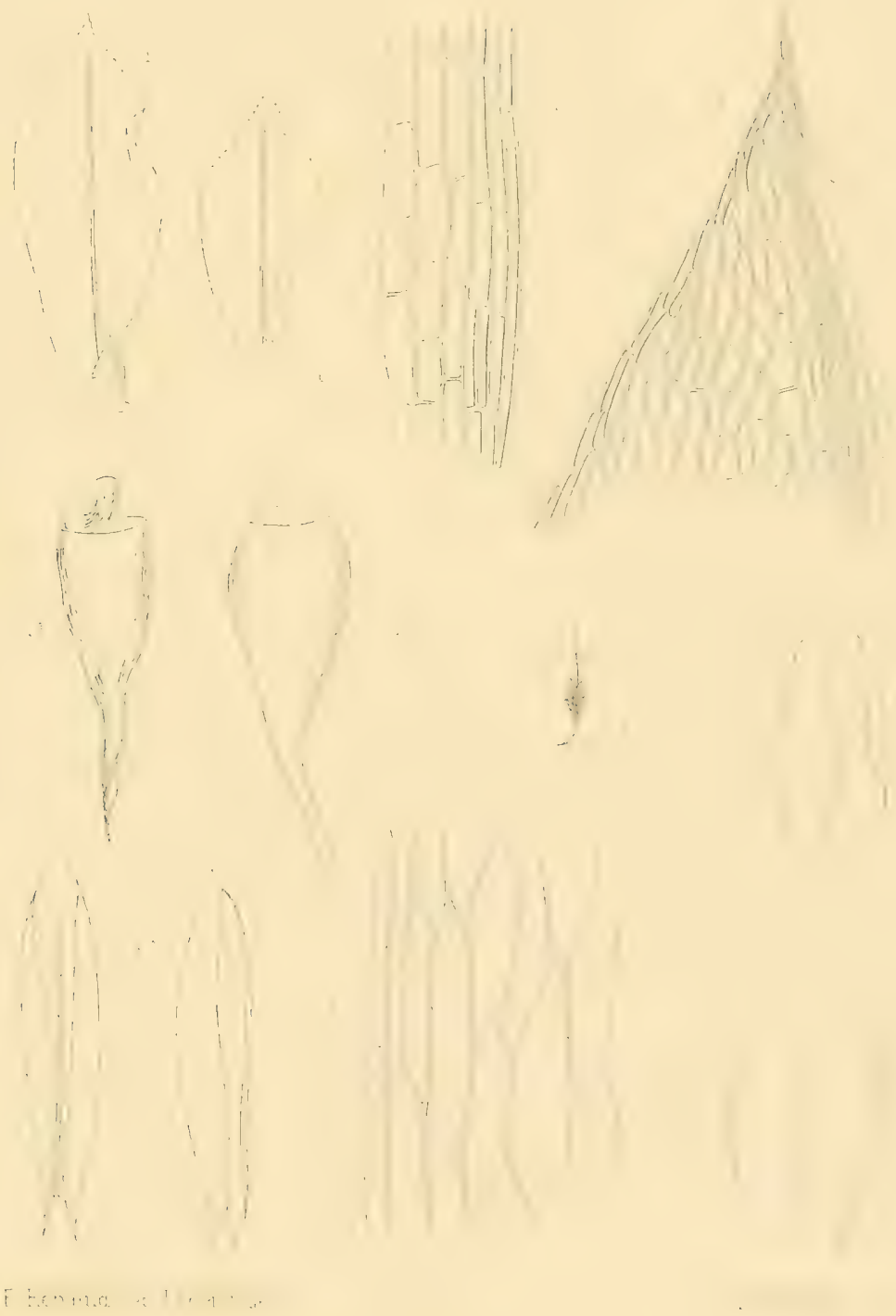






\section{PLANCHE XXIV}

I. Leucoloma Garnieri Par. Ren.

a Feuille caulinaire $\times 24$.

$b$ Nervure vers la base $>$ i 70 .

c Margo hyalin $\times 300$.

2. Leucoloma Cheesemani Ren.

a Feuille caulinaire $\times 24$.

b Oreillette $\times$ i 70 .

c Margo hyalin $\times 300$.

3. Leucoloma subintegrum Broth.

a Oreillette $\times$ I 70 .

4. Leucoloma albulum Sull.

$a$ Feuille caulinaire $\times 24$.

$b$ Oreillette $\times$ i 70 .

c Margo hyalin $\times 300$.

5. Leucoloma subalbulum Ren.

a Feuille caulinaire $>24$.

$b$ Oreillette $\times 170$.

c Margo hyalin $\times 300$. 
Mousses de Madagascar.

i $X \therefore !$

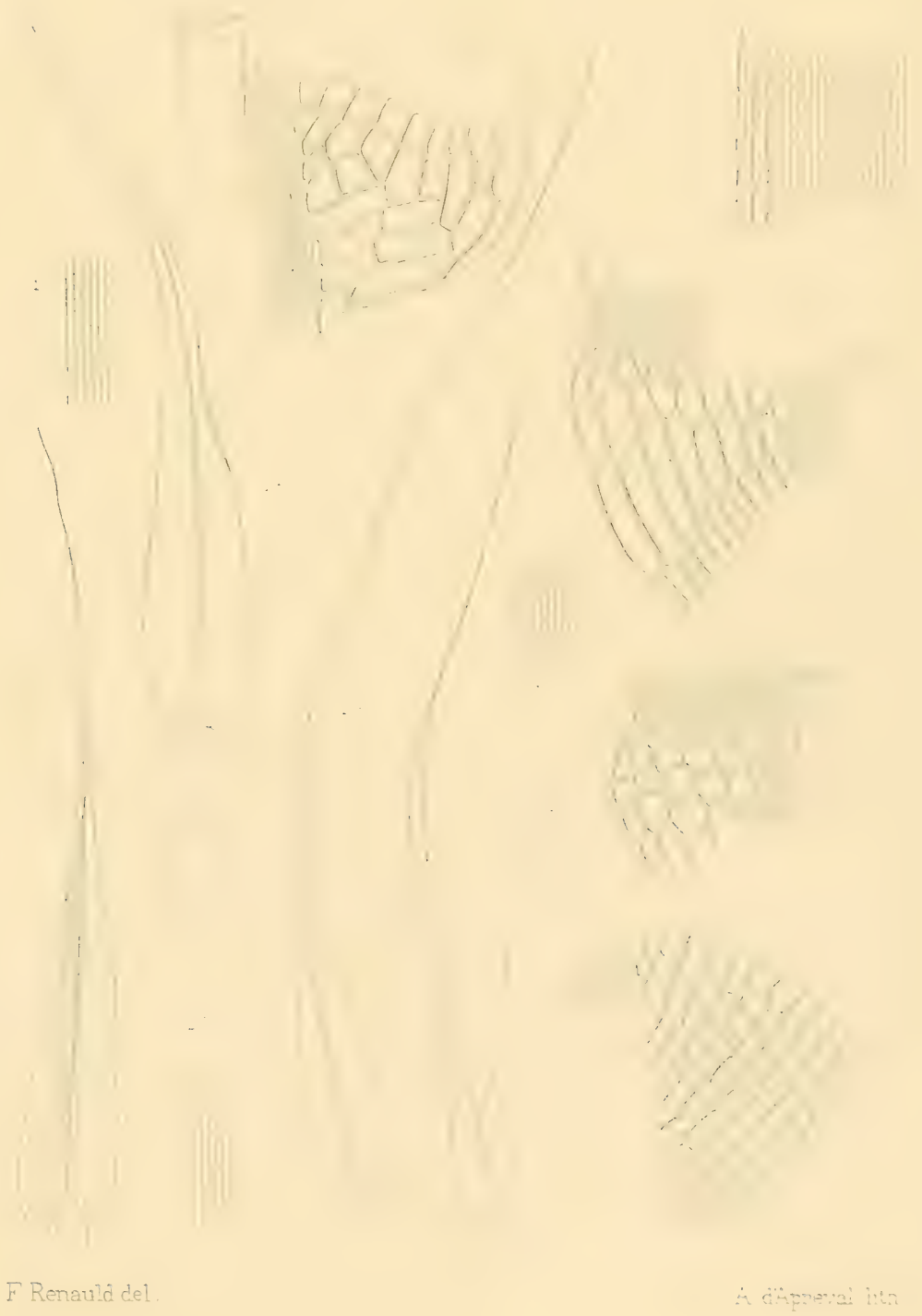






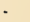





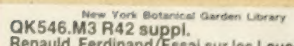




\section{DCCCC IX}

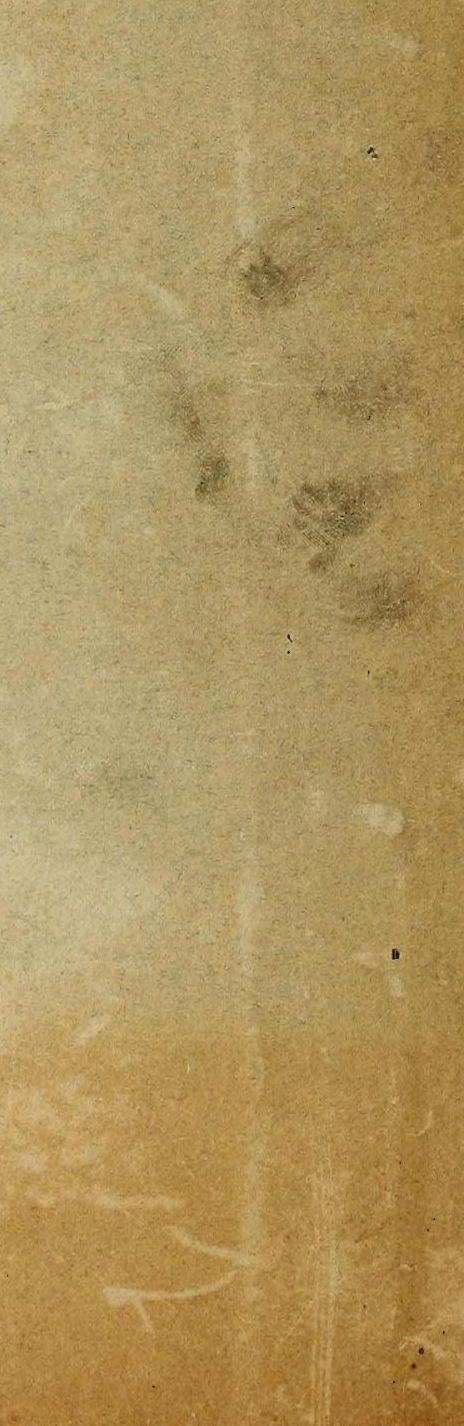

